\title{
The Boundary Element Method
}

in Acoustics

Stephen Kirleup

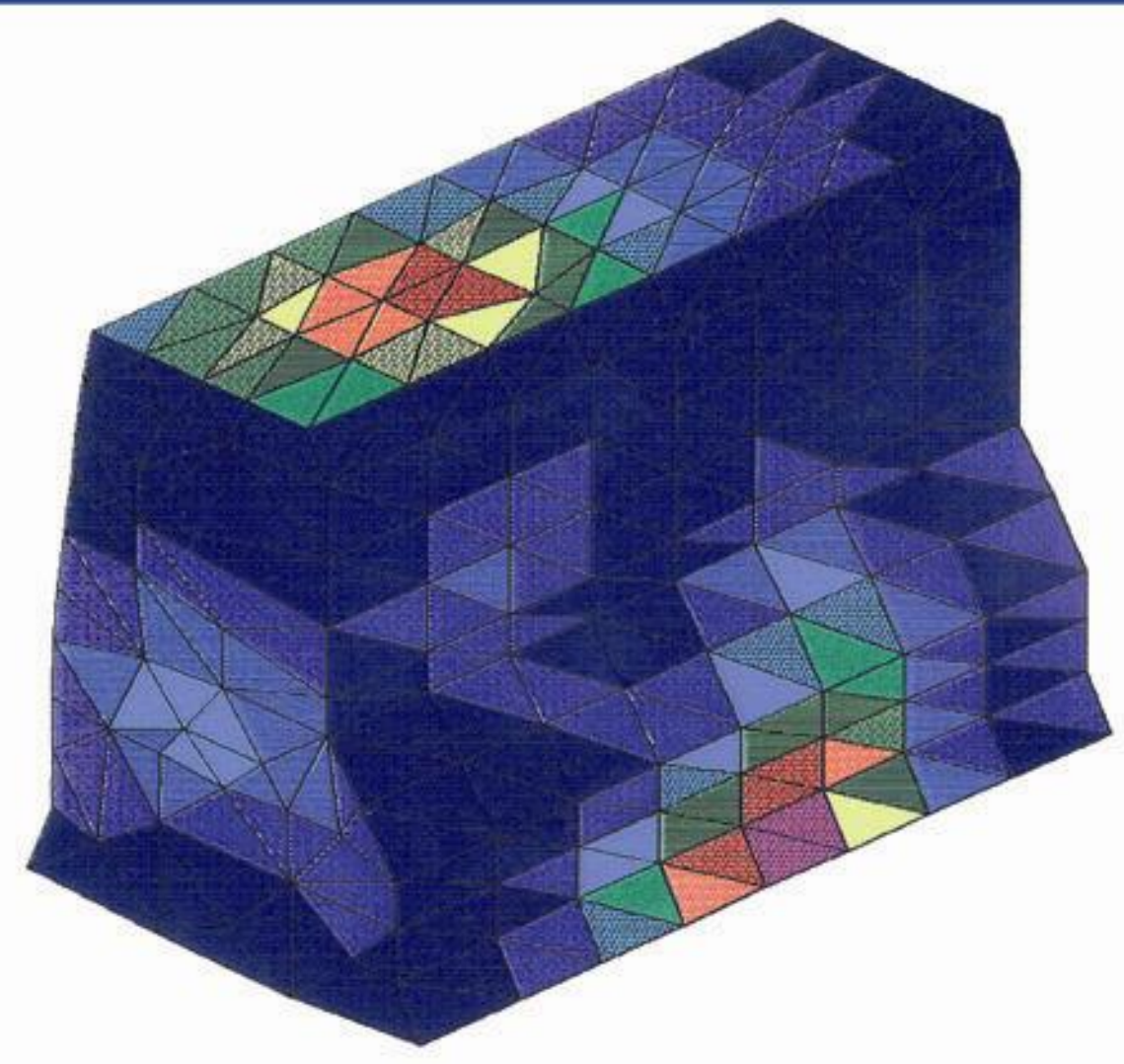

A development in Fortran 
The Boundary Element Method in Acoustics

by

Stephen Kirkup

$1998 / 2007$ 
The Boundary Element Method in Acoustics by S. M. Kirkup

First edition published in 1998 by Integrated Sound Software and is published in 2007 in electronic format (corrections and minor amendments on the 1998 print).

Information on this and related publications and software (including the second edition of this work, when complete) can be obtained from the web site

http://www.boundary-element-method.com

The author's publications can generally be found through the web page

http://www.kirkup.info/papers

The nine subroutines and the corresponding nine example test programs are available in Fortran 77 on CD ABEMFULL. The original core routines can be downloaded from the web site. The learning package of subroutines ABEM2D is can be downloaded from the web site. See the web site for a full price list and alternative methods of obtaining the software.

ISBN 0953403106

The work is subject to copyright. All rights are reserved, whether the whole or part of the material is concerned, specifically the rights of translation, reprinting, reuse of illustrations, recitation, broadcasting, reproduction on microfilm or in any other way, and storage in data banks. Duplication of this publication or parts thereof is subject to the permission of the author.

(c) Stephen Kirkup 1998-2007. 


\section{Preface to 2007 Print}

Having run out of hard copies of the book a number of years ago, the author is pleased to publish an on-line version of the book in PDF format. A number of minor corrections have been made to the original, following feedback from a number of readers. The author is particularly grateful to Ambrose Thompson and Peter Juhl for their feedback.

There have obviously been a number of advances since the publication of the first print in 1998. The author has created the website www.boundary-element-method.com as a general resource on the method. Further developments and applications of the work can be found in the Addendum at the back of this book or on the webpage www.boundary-element-method.com/extra. 


\section{Preface to 1998 Print}

The boundary element method (BEM) is a powerful computational technique, providing numerical solutions to a range of scientific and engineering problems. To the user, the main characteristic of the method is that only a mesh of the boundary of the domain is required. Hence, at the very least, the method is easier to apply than the more traditional finite element method.

The subject of this text is the development of boundary element methods for the solution of problems in linear acoustics. Three classes of problem are considered: Chapter 4 - interior acoustic problems, Chapter 5 - exterior acoustic problems and Chapter 6 - the modal analysis of an enclosed fluid. Each class of problem is considered in three domain settings: two-dimensions, three-dimensions and axisymmetry.

In the field of linear acoustics, the BEM is and important alternative to the more traditional methods. This is especially true for exterior problems, where the acoustic domain such as the open air or the ocean is so large it is acceptable to model it to be infinite in extent. For example consider a vibrating body, radiating into the open air. Applying domain methods, such as the finite element method, to such a problem clearly requires some careful thought. However, it is natural to use the BEM in this kind of application since only a mesh of the surface of the body is required; reducing both the time for preparing the mesh and in the computation of the numerical solution.

In order to apply the boundary element method, the partial differential equation governing the domain (here the Helmholtz or reduced wave equation) must be reformulated as an integral equation relating functions defined on the boundary of the domain only. By representing boundary or surface as a set of panels and the boundary functions by a simple parametric form on each panel, the boundary integral equation is reduced to a linear system of equations and a numerical solution becomes possible. The general development of the BEM and its application in acoustics is considered in the introductory chapter.

In this text the boundary element methods for the solution of acoustic or Helmholtz problems are developed in a bottom-up way. Chapter 2 is on the preparation of the boundary and surface meshes. Chapter 3 considers the techniques employed in reducing the integral equation to discrete form. The solution of the acoustic problems are considered in Chapters 4 to 6 . 
The boundary element method for the solution of acoustic problems has been developed over the last three or four decades. Out of the three problem classes considered, only the interior problem has been found to be straightforward. The development of the BEMs for the exterior problem and the interior modal analysis problem have been hampered my a range of well-publicised difficulties. However, as we shall see in this text, the BEM has reached a level of maturity in these areas that demonstrates that many of these difficulties have been overcome. In this text the development of the BEM for acoustic problems is treated positively.

A library of Fortran 77 routines have been developed alongside this text. Each acoustic problem considered in this text can be solved through simply calling one Fortran routine. The user must simply set up the parameter list for the subroutine in order to obtain the numerical solution by the BEM and to interpret the results. The source code is made available so that the methods employed are entirely transparent. Although the mathematical development of the methods is given in this text, the software can be used without any knowledge of these details. Users of the software will need to have a working knowledge of Fortran.

In the penultimate section of chapters 4 to 6 a range of test problems and results have been given. The source code implementing the test problems and calling the relevant routines is given. Hence the user can view and run the programs, modify and re-run them to build up confidence before going on to more interesting applications. At the end of each of chapters 4 to 6 a typical real-world application of the subroutines has been included to show the wide-ranging potential of the BEM in this application.

A website http://www.boundary-element-method.com has been introduced to provide a wider resource on the BEM in acoustics. The author is interested to hear from readers and users of the software, particularly of ideas for extending this work or of novel applications. The e-mail address is stephen@kirkup.info.

Finally, the author is grateful for all those who have helped him generate the material of this book. In particular David Henwood, George Symm, Geoff Miller, Dick Tyrrell, Sia Amini and Mark Jones. 


\section{Contents}

1 Introduction 1

1.1 The Boundary Element Method in Acoustics . . . . . . . . . . . . . 2

1.2 Outline of The Boundary Element Method . . . . . . . . . . . . . . 4

1.2.1 Operator Notation . . . . . . . . . . . . 6

1.2.2 Numerical Solution of the Integral Equation . . . . . . . . . . 6

1.2 .3 Domain Solution . . . . . . . . . . . . . . . . . 8

1.2.4 Direct and Indirect Boundary Integral Equations . . . . . . . 9

1.2.5 Collocation .................... . . 9

1.3 Acoustics and the Helmholtz Equation . . . . . . . . . . . . . 11

1.3.1 The Wave Equation and the Helmholtz Equation . . . . . . . 12

1.3.2 Other Acoustic Properties (Exterior Problems) . . . . . . . . 13

1.3.3 Acoustic Domains and Conditions . . . . . . . . . . . . . . 14

1.3.4 Condition at Infinity for Exterior Problems . . . . . . . . . . . 14

1.3.5 Resonant Frequencies and Mode Shapes . . . . . . . . . . . 15

1.3.6 Units of Measurement . . . . . . . . . . . . . . . . 15

1.3.7 Acoustic Media: Air and Water . . . . . . . . . . . . 16

1.4 Computer Programming . . . . . . . . . . . . . . 16

1.4.1 Fortran .................... 17

1.4 .2 Extending the work . . . . . . . . . . . . . . 17

1.4.3 Subordinate routines ................ . . . 18 
1.4.4 Computational Efficiency . . . . . . . . . . . . . . . . 19

2 Boundary Representation $\quad 21$

2.1 Subdivision of the Boundary into Panels . . . . . . . . . . . 21

2.2 Two dimensional Boundaries . . . . . . . . . . . . . . . . . . . 22

2.3 Three dimensional Surfaces _ . . . . . . . . . . . . . . 25

2.4 Axisymmetric Surfaces . . . . . . . . . . . . . . . . 26

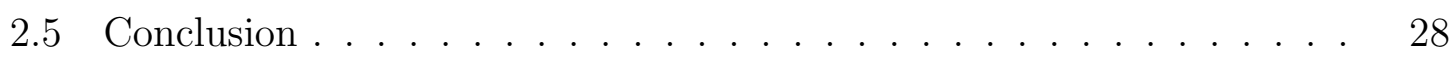

3 The Discrete Helmholtz Operators $\quad 31$

3.1 The Helmholtz Integral Operators . . . . . . . . . . . . . . . . 32

3.1 .1 The Helmholtz Operators . . . . . . . . . . . . . . . 32

3.1 .2 Green's functions . . . . . . . . . . . . . . . . . . 32

3.1 .3 Properties of the Operators . . . . . . . . . . . . 33

3.2 Some Properties of the Kernel Functions . . . . . . . . . . . . . 33

3.2.1 Derivatives of $G_{0}$ with respect to $r \ldots \ldots \ldots$

3.2.2 Derivatives of $G_{k}(k \neq 0)$ with respect to $r \ldots \ldots 34$

3.2.3 Expressions for the normal derivatives of $G_{k} \ldots \ldots \ldots$

3.2.4 Expressions for the normal derivative of $r \ldots \ldots 34$

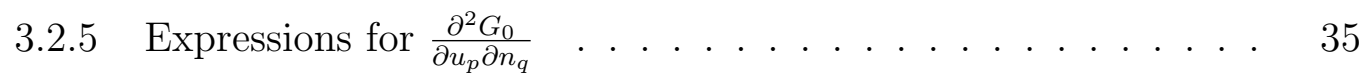

3.2 .6 Asymptotic Properties . . . . . . . . . . . . . . . . 35

3.3 Discretisation . . . . . . . . . . . . . . . . . . 35

3.4 Evaluation of the Discrete Forms $\ldots \ldots \ldots \ldots$

3.5 General Introduction to the Subroutines $\ldots \ldots \ldots$

3.5.1 General control of the subroutines . . . . . . . . . . . 39

3.5 .2 Geometrical Information . . . . . . . . . . . . . . 39

3.5 .3 Quadrature Rule . . . . . . . . . . . . . . . . . 40

3.5.4 Validation of the input . . . . . . . . . . . . 42

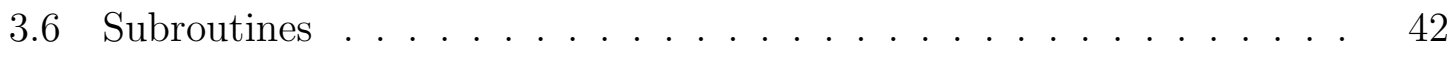




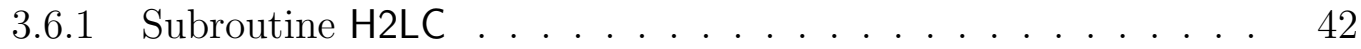

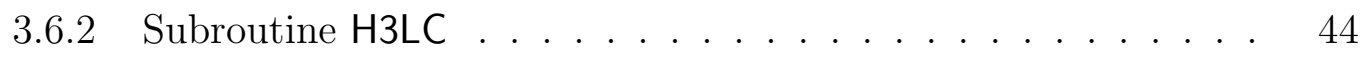

3.6.3 Subroutine H3ALC . . . . . . . . . . . . . . . . 46

3.7 Special Numerical Integration Methods . . . . . . . . . . . . . . . . 48

3.7.1 Two-dimensional problems . . . . . . . . . . . . . . . . . 49

3.7.2 Three-dimensional problems . . . . . . . . . . . . . 49

3.7.3 Axisymmetric three-dimensional problems . . . . . . . . . 51

3.8 Analysis of Computational Cost . . . . . . . . . . . . . . . 51

3.9 Conclusion . . . . . . . . . . . . . . . . . . . . . . . . . . . . . . 54

4 The Interior Acoustic Problem 55

4.1 Integral Equation Formulation $\ldots \ldots \ldots \ldots \ldots$

4.1 .1 Direct Formulation . . . . . . . . . . . . . . . . 57

4.1 .2 Indirect Formulation . . . . . . . . . . . . . . . . 58

4.1 .3 Field Modification . . . . . . . . . . . . . . . . . . 59

4.2 Boundary Element Method . . . . . . . . . . . . . . . . . . . . 61

4.2.1 Direct Boundary Element Method . . . . . . . . . . . . . 61

4.2.2 Indirect Boundary Element Method . . . . . . . . . . . . . 62

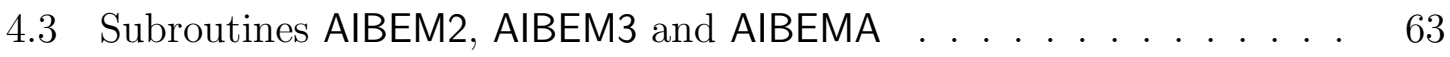

4.3.1 Solution Strategy of the AlBEM* routines _ . . . . . . 63

4.3.2 Subroutine AlBEM2 . . . . . . . . . . . . . . . . . 64

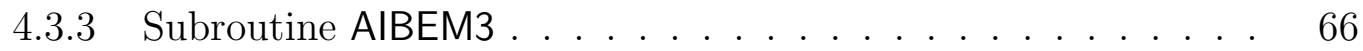

4.3.4 Subroutine AIBEMA . . . . . . . . . . . . . . . 69

4.4 Test Programs . . . . . . . . . . . . . . . . . . . . . 71

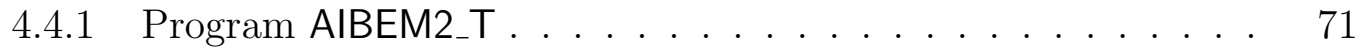

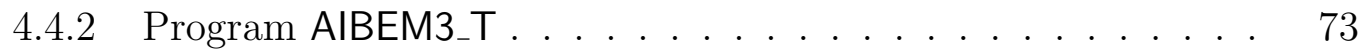

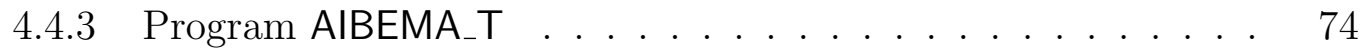

4.5 Application: Interior acoustics of a $2 \mathrm{D}$ car $\ldots \ldots \ldots \ldots$ 
4.6 Conclusion . . . . . . . . . . . . . . . . . . . . . . . . . 77

5 The Exterior Acoustic Problem $\quad 79$

5.1 Elementary Formulations and Methods . . . . . . . . . . . . 81

5.1.1 Elementary Direct Formulations . . . . . . . . . . . . . . 81

5.1.2 Elementary Indirect Formulations . . . . . . . . . . . . . . 82

5.1 .3 Elementary Methods . . . . . . . . . . . . . . . . . . . 82

5.2 The Schenck Method . . . . . . . . . . . . . . . . . . . 84

5.3 Improved Formulations . . . . . . . . . . . . . . . 85

5.3.1 Improved Indirect Formulation $\ldots \ldots \ldots \ldots$

5.3.2 Improved Direct Formulation _ . . . . . . . . . . . . 87

$5.3 .3 \quad$ Scattering . . . . . . . . . . . . . . . . . . 87

5.4 Improved Boundary Element Methods . . . . . . . . . . . . . . 88

5.4 .1 Improved Indirect Method . . . . . . . . . . . . . . 89

5.4 .2 Improved Direct Method . . . . . . . . . . . . . . . . . . 89

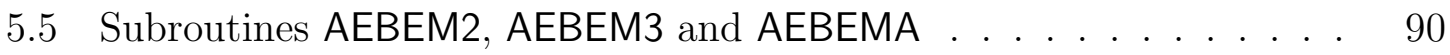

5.5.1 Solution Strategy of the AEBEM* routines . . . . . . . . 91

$5.5 .2 \quad$ Subroutine AEBEM2 . . . . . . . . . . . . . . . . 91

5.5 .3 Subroutine AEBEM3 . . . . . . . . . . . . . . . . . 94

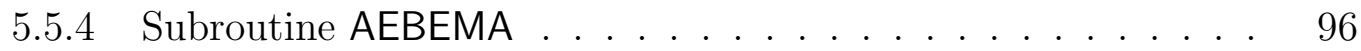

$5.6 \quad$ Test $\operatorname{Programs} \ldots \ldots \ldots \ldots$

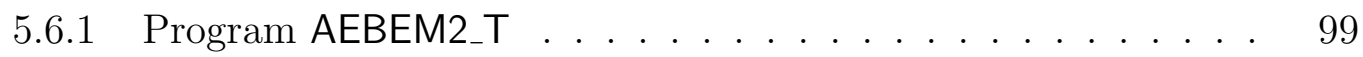

$5.6 .2 \quad$ Program AEBEM3_T . . . . . . . . . . . . . . . . 100

5.6 .3 Program AEBEMA_T . . . . . . . . . . . . . . . 101

5.7 Application: Engine Noise Analysis . . . . . . . . . . . . . . . 103

5.7 .1 Details . . . . . . . . . . . . . . . . . . . . 103

5.7 .2 Results . . . . . . . . . . . . . . . . . . . . . . . 103

5.8 Conclusion . . . . . . . . . . . . . . . . . . . . . . . . . . . . . . . . . . . . 104 
6 Interior Modal Analysis 107

6.1 Formulations of the Eigenvalue Problem . . . . . . . . . . . . . 109

6.1.1 Indirect Formulation . . . . . . . . . . . . . . . 109

6.1 .2 Direct Formulation . . . . . . . . . . . . . . . . . . 110

6.2 Application of Collocation . . . . . . . . . . . . . . . . . 110

6.2.1 Indirect Method . . . . . . . . . . . . . . . . . 110

6.2.2 Direct Method . . . . . . . . . . . . . . 111

6.3 Solution of the Non-linear Eigenvalue Problem . . . . . . . . . . . . . 111

6.4 Subroutines MBEM2, MBEM3 and MBEMA . . . . . . . . . . . . . . 112

6.4.1 Solution Strategy of the MBEM* routines . . . . . . . . . . . . 113

6.4.2 Subroutine MBEM2 . . . . . . . . . . . . 113

6.4.3 Subroutine MBEM3 . . . . . . . . . . . . 116

6.4.4 Subroutine MBEMA . . . . . . . . . . . . . . 119

6.5 Test problems . . . . . . . . . . . . . . . . . . . 122

6.5.1 Program MBEM2_T . . . . . . . . . . . . . 122

6.5.2 Program MBEM3_T . . . . . . . . . . . . . . 123

6.5.3 Program MBEMA_T .................... 123

6.6 Application: Loudspeaker Enclosure . . . . . . . . . . . . . . . . 124

6.6.1 Background ....................... 124

6.6.2 Particular implementations of computational method and Measurements . . . . . . . . . . . . . . . . 126

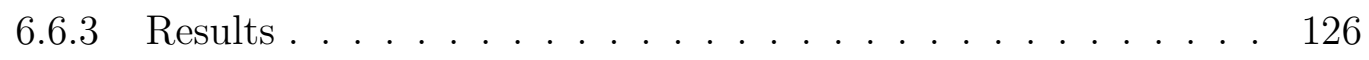

6.7 Conclusion . . . . . . . . . . . . . . . . . . . 128 


\section{Chapter 1}

\section{Introduction}

Over recent decades, the boundary element method (BEM) has received much attention from researchers and has become an important technique in the computational solution of a number of physical problems. In common with the better-known finite element method (FEM) and finite difference method (FDM), the boundary element method is essentially a method for solving partial differential equations (PDEs) and can only be employed when the physical problem can be expressed as such. As with the other methods mentioned, the boundary element method is a numerical method and hence it is an important subject of research amongst the numerical analysis community. However, the potential advantages of the BEM have seemed so considerable that the strongest impetus behind its development has come from the engineering community, in its enthusiasm to obtain flexible and efficient computer-based solutions to a range of engineering problems. The boundary element method has found application in such diverse topics as stress analysis, potential flow, fracture mechanics and acoustics (the subject of this text).

Acoustics is an important branch of physical science. An acoustic field can exist in a fluid domain such as air or water, the two most important acoustic media. The linear wave equation forms an acceptable model in many fluids and it is often used in the cases of air and water media. In many physical situations the acoustic field is periodic, and has the outcome of reducing the wave equation to a sequence of Helmholtz equations by a Fourier decomposition with one Helmholtz equation for each sample frequency. In the methods of this text, solutions of such acoustic problems are obtained through the consideration of the individual Helmholtz problems.

The Helmholtz equation governing a range of classes of domains may be solved by the boundary element method. Hence the BEM has received attention from engineers 
that are interested in applications such as the sound output of a loudspeaker, the noise from a radiating source such as an engine and the interior acoustic modes of an enclosure such as a vehicle interior. The method is equally applicable in underwater acoustics and can be used to model the scattering effect of an obstruction in the ocean or to determine the acoustic field surrounding a sonar transducer.

Here the development of the boundary element method for a range of acoustic domains and conditions is presented. Fortran subroutines that implement the methods and a set of test problems are available. A chapter is devoted to each of the interior, exterior and modal acoustic problems. The codes implement the boundary element method in its simplest form. The boundaries are approximated by the straight lines for two dimensional problems, planar triangles for three-dimensional problems and by truncated cones in axisymmetric three-dimensional problems. The boundary functions are approximated by a constant on each segment or panel of the boundaries. Specif-

ically, the boundary elements are $C^{-1}$; constant on each panel and discontinuous on the edges of the panels.

The subroutines have been designed to allow as much flexibility as possible whilst minimizing their complexity. The parameter list in each main subroutine is stated in the text to enable the reader to appreciate the way in which a call to the subroutine can be included in a Fortran program. Toward the end of Chapters 4, 5 and 6 test problems are applied to each subroutine, to demonstrate how to use the routine and to validate the methods. Results from relevant applications are given to demonstrate the usefulness of the methods in the computer-aided analysis of engineering problems.

\subsection{The Boundary Element Method in Acoustics}

The boundary element method is derived through the discretisation of an integral equation that is mathematically equivalent to the original partial differential equation. The essential re-formulation of the PDE that underlies the BEM consists of an integral equation that is defined on the boundary of the domain and an integral that relates the boundary solution to the solution at points in the domain. The former is termed a boundary integral equation (BIE) and the BEM is often referred to as the boundary integral equation method or boundary integral method. Over the last twenty years the term boundary element method has become more popular. The other terms are still used in the literature however, particularly when authors wish to refer to the overall derivation and analysis of the methods, rather than their implementation or application. 
An integral equation re-formulation can only be derived for certain classes of PDE. Hence the BEM is not widely applicable when compared to the near-universal adaptability of the finite element and finite difference method. However, in the cases in which the boundary element method is applicable, it often results in a numerical method that is easier to use and more computationally efficient than the competing methods.

The advantage in the boundary element method arises from the fact that only the boundary (or boundaries) of the domain of the PDE requires sub-division. (In the finite element method or finite difference method the whole domain of the PDE requires discretisation.) Thus the dimension of the problem is effectively reduced by one, for example an equation governing a three-dimensional region is transformed into one over its surface. In cases where the domain is exterior to the boundary, as it is in acoustic radiation and scattering models, the extent of the domain is infinite and hence the advantages of the BEM are even more striking; the equation governing the infinite domain is reduced to an equation over the (finite) boundary.

Solutions to two-dimensional, three-dimensional and axisymmetric three-dimensional acoustic problems are developed in this text. In each case boundary element techniques are developed for the solution of interior and exterior boundary-value problems and the interior eigenvalue problem, each allowing for a wide range of classes of boundary condition. The subroutines have been written so that they are applicable to a wide variety of acoustic problems. A very general form of boundary condition is assumed and it is possible to include an incident field that is necessary in acoustic scattering, for example. Nine major Fortran subroutines are developed for solving each of these problems and the source codes are available. It is the emphasis on the method development and programming that distinguishes this work from earlier texts such as Colton and Kress [23], Ciskowski and Brebbia [22] and Rego Silva [72].

\begin{tabular}{|c|c|c|c|c|}
\hline \multicolumn{5}{|c|}{ Table 1.A: The main subroutines } \\
\hline & Chapter & 2-dimensional & 3-dimensional & Axisymmetric \\
\hline Core routines & 3 & H2LC & H3LC & H3ALC \\
\hline Interior analysis & 4 & AIBEM2 & AIBEM3 & AIBEMA \\
Exterior analysis & 5 & AEBEM2 & AEBEM3 & AEBEMA \\
Modal analysis & 6 & MBEM2 & MBEM3 & MBEMA \\
\hline
\end{tabular}

The three subroutines, for each dimensional space, call a core routine that is responsible for discretising the integral equation. Chapter 3 decribes the core routines; the 
underlying methods employed and the way in which the routines are called from the main programs. The major subroutines supplied with the text are listed in Table 1.A. In science and engineering there are a wide variety of acoustic problems, only a fraction of which are considered in this work. The important example of fluid-structure interaction modelling, in which an acoustic field existing in a fluid influences and is influenced by a structure with which it is in contact [83], [59], [3] and [42], is not addressed in this work. However, the subroutines described in Chapters 4-6 can be adapted to include such problems.

A further important extension of the methods in this text is the use of integral equation methods for modelling the acoustic field exterior to a thin shell or shield. The traditional boundary element method is not directly applicable to such problems and alternative integral equation methods have been developed in references [10], [82]. This method has been developed as a generalisation of the boundary element method (the boundary and shell element method) by the author [47], [41], [49]. Again such problems are beyond the scope of this text. More advanced use of the subroutines is discussed in Appendix 7.

\subsection{Outline of The Boundary Element Method}

In this Section the overall strategy in using the BEM is briefly outlined. For a more thorough introduction, the reader is advised to consult the textbooks exclusively devoted to the boundary element method, such as Jaswon and Symm [34], Brebbia [13], Banerjee and Butterfield [7], Chen and Zhou [20] and Hall [29]. An introduction

to the boundary element method with subroutines for solving Laplace's equation is also available on the web site [33].

In the derivation of the BEM, the underlying objective is to replace the partial differential equation that governs the solution in a domain by an equation that governs the solution on the boundary alone. For example the Laplace equation

$$
\sum_{i=1}^{N} \frac{\partial^{2} \varphi(\mathbf{p})}{\partial p_{i}^{2}}=0
$$

where $N$ is the dimension of the space, or more concisely,

$$
\nabla^{2} \varphi(\mathbf{p})=0
$$

governing the interior to a domain $D$ bounded by a surface $S$ (as shown in Figure 
1.1) can be replaced by an integral equation of the form

$$
\int_{S} \frac{\partial G}{\partial n_{q}}(\mathbf{p}, \mathbf{q}) \varphi(\mathbf{q}) d S_{q}+\frac{1}{2} \varphi(q)=\int_{S} G(\mathbf{p}, \mathbf{q}) \frac{\partial \varphi}{\partial n_{q}} d S_{q} .
$$

The function $G$ is known as a Green's function, the precise form of which for the Laplace (and Helmholtz) equation is given in Section 3.1. Physically, $G(\mathbf{p}, \mathbf{q})$ represents the effect observed at a point $\mathbf{p}$ of a unit source at the point $\mathbf{q}$. The terminolology $\frac{\partial *}{\partial n_{q}}$ represents the partial derivative of the function $*$ with respect to the unit outward normal at the point $\mathbf{q}$ on the boundary.

The integral equation can be derived from the Laplace equation by applying Green's second theorem. The power of the formulation (1.1) lies in the fact that it relates the potential $\varphi$ and its derivative on the boundary alone; no reference is made to $\varphi$ at points in the domain. In a typical boundary-value problem we may be given $\varphi(\mathbf{q})$, $\frac{\partial \varphi}{\partial n_{q}}(\mathbf{q})$ or a combination of such data on $S$ : equation (1.1) is a means of determining the unknown boundary function(s) from given boundary data.

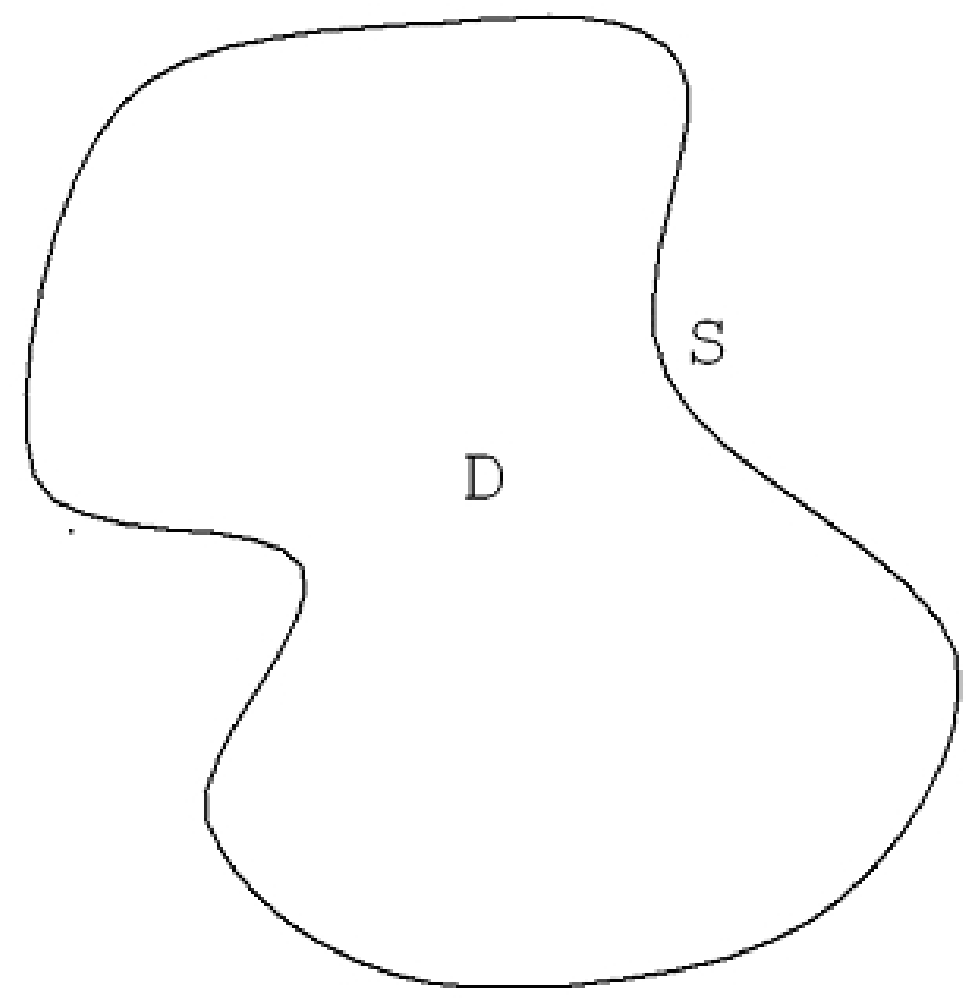

Fig. 1.1. Illustration of the interior domain. 


\subsubsection{Operator Notation}

Operator notation is a useful shorthand in writing integral equations. Moreover, it will be shown that it is very powerful notation in that it clearly demonstrates the connection between the integral equation and the linear system of equations that results from its discretisation.

Integral equations can always be written in terms of integral operators. For example if $\zeta$ is a function defined on $S$ then applying the following operation to $\zeta$ for all points p on $S$

$$
\int_{S} G(\mathbf{p}, \mathbf{q}) \zeta(q) d S_{q}=\nu(\mathbf{p}) \quad(\mathbf{p} \in S)
$$

gives a function $\nu$. This can be viewed as the application of an operator to the function $\zeta$ to return the function $\nu$. More simply we may write

$$
\{L \zeta\}_{S}(\mathbf{p})=\nu(\mathbf{p})
$$

In (1.2) the $L$ represents the integral operator

and the subscript $(\Gamma)$ refers to the domain of integration. Here $\Gamma$ is used as a variable, representing either a whole surface or a patch of surface.

In operator notation the integral equation (1.1) can be written in the alternative shorthand notation

$$
\{M \varphi\}_{S}(\mathbf{p})+\frac{1}{2} \varphi(\mathbf{p})=\{L v\}_{S}(\mathbf{p}) \quad \text { or } \quad\left\{\left(M+\frac{1}{2} I\right) \varphi\right\}_{S}(\mathbf{p})=\{L v\}_{S}(\mathbf{p})
$$

where $v(\mathbf{q})=\frac{\partial \varphi}{\partial n_{q}}$, the $M$ represents the other integral operator;

$$
\{M \zeta\}_{\Gamma}(\mathbf{p}) \equiv \int_{\Gamma} \frac{\partial G}{\partial n_{q}}(\mathbf{p}, \mathbf{q}) \zeta(\mathbf{q}) d S_{q}
$$

and $I$ the identity operator.

\subsubsection{Numerical Solution of the Integral Equation}

In order to develop a numerical method for the solution of integral equations like (1.3), a technique is applied so that the equation is simplified into a linear system of equations. Hence there is a close analogy between linear integral equations and systems of linear equations; the integral operators can be viewed as matrices, the boundary functions as vectors. 
The application of such a technique transforms the equation (1.3) to an equation of the form

$$
\mathrm{M} \underline{\hat{\varphi}}+\frac{1}{2} \underline{\hat{\varphi}}=\mathrm{L} \underline{\hat{v}} \quad \text { or } \quad\left(\mathrm{M}+\frac{1}{2} \mathrm{I}\right) \underline{\hat{\varphi}}=\mathrm{L} \underline{\hat{v}}
$$

where the components of the vectors $\underline{\hat{\varphi}}$ and $\underline{\hat{v}}$ represent (approximations to) the values of the function $\varphi(\mathbf{p})$ and $\frac{\partial \varphi}{\partial n_{q}}(\mathbf{p})$ at a set of points on the boundary. L, M and I are matrices derived from the corresponding integral operators in (1.3) with I representing the identity matrix. A complete demonstration of how the matrix-vector equation is obtained from the integral equation in given in Subsection 1.2.5. The connection between the system of linear equations (1.5) and the integral equation (1.3) or (1.1) is now clear. As stated earlier, the boundary data $\varphi, \frac{\partial \varphi}{\partial n_{q}}$ or some combination of the two functions are given and the solution of the system of linear equations (1.5) can be used to derive approximations to the unknown boundary data.

There are a variety of techniques for deriving the system of linear equations from a given integral equation (see [5], [26], for example). In general, a method can be derived by replacing the integrals in an integral equation by a quadrature formula or by a weighted residual method such as the Galerkin method. Many methods for solving integral equation can be used to develop a particular boundary element method [13]. The method employed in the software accompanying this text is that of collocation since it is considerably easier to program and probably more efficient than competing techniques.

The application of collocation to a boundary integral equation requires that the boundary is represented by a set of panels. For example a two dimensional boundary can be approximated by a set of straight lines as illustrated in Figure 1.2. In order to complete the discretisation of the integral equations, the boundary functions also need to be approximated on each panel. In this work, it is the characteristics of the panel and the representation of the boundary function on the panel that together define the element in the boundary element method. The technique of dividing the boundaries into panels is described in Chapter 2 .

By representing the boundary functions by a characteristic form on each panel, the boundary integral equations can be written as a linear system of equations of the form introduced earlier. The boundary functions are approximated by a constant on each panel in all of the methods associated with this text, with the collocation point at the centre of the panel ( $C^{-1}$ collocation). The overall process is that of discretising the integral operators and the methods for carrying this out are covered in Chapter 3 . 


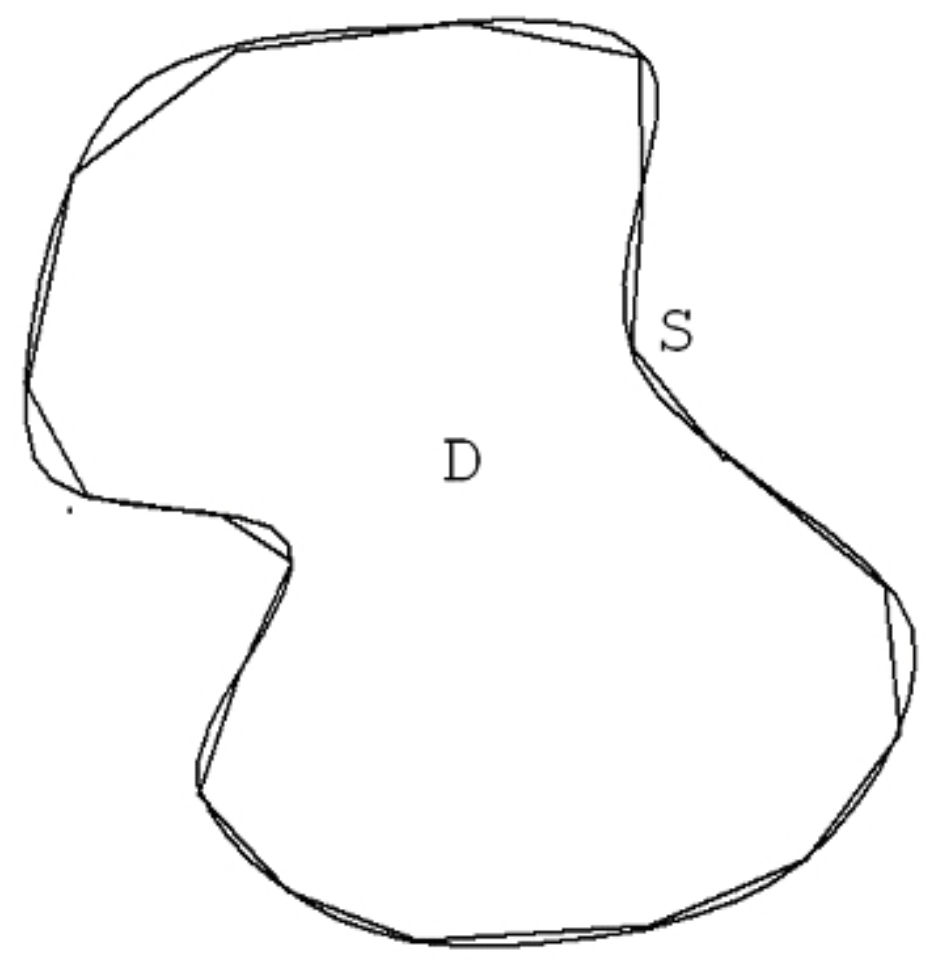

Fig 1.2. The boundary represented by a set of straight line panels.

\subsubsection{Domain Solution}

On solution of the integral equation (approximations to) the unknown boundary function(s) will be known on $S$. Hence both of $\varphi(\mathbf{q})$ and $\frac{\partial \varphi}{\partial n_{q}}(\mathbf{q})$ will be explicit if the method is based on the integral equation (1.5). In most cases a solution in the domain $D$ is required and this can be found using the following equation:

$$
\varphi(\mathbf{p})=\int_{S} G(\mathbf{p}, \mathbf{q}) \frac{\partial \varphi}{\partial n_{q}} d S_{q}-\int_{S} \frac{\partial G}{\partial n_{q}}(\mathbf{p}, \mathbf{q}) \varphi(q) d S_{q} \quad(\mathbf{p} \in D)
$$

or, more concisely,

$$
\varphi(\mathbf{p})=\{L \varphi\}_{S}-\{M v\}_{S} \quad(\mathbf{p} \in D)
$$

using the notation of Subsection 1.2.1. The equation (1.6) is also an outcome of Green's second theorem. The domain solution $\varphi(\mathbf{p})$ for any point $\mathbf{p}$ in the domain can be obtained through simply evaluating the integrals in (1.6). 


\subsubsection{Direct and Indirect Boundary Integral Equations}

There are two fundamental approaches to the derivation of an integral equation formulation of a partial differential equation. The first is often termed the direct method and the integral equations are derived through the application of Green's second theorem and an example of this has already been given in Subsection 1.2.1.

The other technique is termed the indirect method. This is based on the assumption that the solution can be expressed in terms of a source density function defined on the boundary. For example it is assumed that the solution of the Laplace equation can be written in the form

$$
\varphi(\mathbf{p})=\int_{S} G(\mathbf{p}, \mathbf{q}) \sigma(\mathbf{q}) d S_{q},
$$

where $\sigma$ is the source density function defined on $S$ only. The following integral equation can be derived from the above:

$$
\frac{\partial \varphi}{\partial n_{p}}(\mathbf{p})=\frac{\partial}{\partial n_{p}} \int_{S} G(\mathbf{p}, \mathbf{q}) \sigma(\mathbf{q}) d S_{q}+\frac{1}{2} \sigma(\mathbf{p})=\int_{S} \frac{\partial G}{\partial n_{p}}(\mathbf{p}, \mathbf{q}) \sigma(\mathbf{q}) d S_{q}+\frac{1}{2} \sigma(\mathbf{p}) .
$$

In operator notation the above integral equations are written

$$
\varphi(\mathbf{p})=\{L \sigma\}(\mathbf{p}) \quad \text { and } \quad v(\mathbf{p})=\left\{\left(M^{t}+\frac{1}{2} I\right) \sigma\right\}(\mathbf{p}) .
$$

Note the relationship between the operator $M^{t}$ and the operator $M$ introduced earlier; $M^{t}$ is known at the transpose of $M$ and is arrived at simply by swapping the arguments $\mathbf{p}$ and $\mathbf{q}$ in the definition.

In some cases the indirect method is a more versatile reformulation of the partial differential equation as the terms $\varphi$ and $\frac{\partial \varphi}{\partial n}$ are already isolated; the expressions for $\varphi$ and $v$ can be substituted directly into the boundary condition, giving the indirect integral equation formulation. For a general boundary condition, the direct method is a little more difficult to implement. In this text both the direct and indirect formulations and methods for solving Helmholtz problems are considered. However, the accompanying main subroutines all implement direct methods.

\subsubsection{Collocation}

The step from the integral equation to the linear system of equations, as illustrated in Section 1.2, is carried out by applying an integral equation method to an equation such as (1.3) to give an equation like (1.5). There are a range of methods for carrying 
this out (ref [13]) but the most favoured technique is that of collocation, because of its inherent simplicity. Collocation can be applied in a remarkably elementary form, which is termed $\mathrm{C}^{0}$ collocation in this text since it is derived by approximating the boundary functions by a constant on each panel. In this subsection the $\mathrm{C}^{0}$ collocation method is briefly outlined.

To begin with the boundary $S$ is assumed to be expressed as a set of panels; $S=$ $\sum_{j=1}^{n} \Delta S_{j}$. Usually the panels have a characteristic form and cannot represent a given boundary exactly, but this distinction will be left for the next chapter. Often the $\Delta S_{j}$ are referred to as elements in other texts. However, the term element refers not only to the geometry of $\Delta S_{j}$ but also to the method of representing the boundary functions on $\Delta S_{j}$. The representation of boundaries in two-dimensional space, three-dimensional space and axisymmetric problems is considered in Chapter 2.

The $\mathrm{C}^{0}$ collocation method involves representing the boundary function by a constant on each panel. For example

$$
\varphi(\mathbf{p}) \approx \varphi_{j}, \quad v(\mathbf{p}) \approx v_{j} \text { if } \mathbf{p} \in \Delta S_{j}
$$

The substitution of representations of this form for the boundary functions in the integral equation reduce it to discrete form. The combination of the representation of the panels and the approximation of the boundary functions, as typified by (1.7), defines the element.

The simplifications allow us to re-write equation (1.3) as the approximation

$$
\sum_{j=1}^{n}\left\{\left(M+\frac{1}{2} I\right) e\right\}_{\Delta S_{j}}(\mathbf{p}) \varphi_{j} \approx \sum_{j=1}^{n}\{L e\}_{\Delta S_{j}}(\mathbf{p}) v_{j} \quad(\mathbf{p} \in S)
$$

where $e$ is the unit function $(e \equiv 1)$. The $\{L e\}_{\Delta S_{j}}(\mathbf{p})$, for example, for a specific point $\mathbf{p}$, are the numerical values of definite integrals and are termed the discrete form of the $L$ integral operator.

The constant approximation is taken to be the value of the boundary functions at the representative central point (the collocation point) on each panel. By finding the discrete forms of the relevant integral operators for all the collocation points, a system of the form

$$
\sum_{j=1}^{n}\left\{\left(M+\frac{1}{2} I\right) e\right\}_{\Delta S_{j}}\left(\mathbf{p}_{i}\right) \varphi_{j} \approx \sum_{j=1}^{n}\{L e\}_{\Delta S_{j}}\left(\mathbf{p}_{i}\right) v_{j}
$$

for $i=1,2, \ldots, n$ is obtained by putting $\mathbf{p}=\mathbf{p}_{i}$ in the previous approximation. Note that because of the approximation of the boundary functions (and also the boundary approximation, if applicable), the discrete equivalent of equation (1.3) is an approximation relating the exact values of the boundary functions at the collocation points. 
This system of approximations (1.8) can now be written in the matrix-vector form

$$
\left(\mathrm{M}+\frac{1}{2} \mathrm{I}\right) \underline{\varphi} \approx \mathrm{L} \underline{v}
$$

with

$$
[\mathrm{L}]_{i j}=\{L e\}_{\Delta S_{j}}\left(\mathbf{p}_{i}\right), \quad[\mathrm{M}]_{i j}=\{M e\}_{\Delta S_{j}}\left(\mathbf{p}_{i}\right)
$$

The vectors $\underline{\varphi}$ and $\underline{v}$ represent the exact values of $\varphi$ and $v$ at the collocation points. The approximate relationship between the exact values (1.9) can be interpreted as an exact relationship between approximate values, equation (1.5).

The terms on the right hand side of the equations in (1.10) are definite integrals that need to be computed to return their value on the left hand side of the equations. The process of computing the integral is termed the discretisation of the integral operators. In many cases this is carried out through a straightforward application of a numerical integration method. However, in the implementation of boundary element methods it is well-known that some of the integrals that arise have singular integrands, requiring special methods. For Helmholtz problems, some of the integral operators that arise are more difficult still and their discretisation requires careful treatment. The techniques employed to discretise the Helmholtz operators in the boundary element methods of this text are described in Chapter 3.

\subsection{Acoustics and the Helmholtz Equation}

The governing equations in acoustics need to be prepared for the application of the boundary element method. For example the time-dependent sound pressure in the original wave equation model is replaced by a potential in the Helmholtz equation and the BEM is applied to the latter. However, eventually the results from the BEM need to be interpreted in terms of physical acoustic properties - not just the sound pressure but also the sound power, radiation ratio and also often on the decibel scale.

In this Section it is shown how the time-dependent wave equation governing the acoustic field can be simplified to the Helmholtz equation when harmonic solutions are considered. For further background to acoustic properties and acoustic modelling see texts such as Morse [62] or Pierce [71]. The three classes of acoustic problem that form the subject of this text are formally described. The content of this Section assumes more importance towards the end of Chapters 4-6 where test problems and applications of the methods are considered. 


\subsubsection{The Wave Equation and the Helmholtz Equation}

The acoustic field is assumed to be present in the domain of a homogeneous isotropic fluid. Whatever the shape and nature of the domain, the acoustic field is taken to be governed by the linear wave equation

$$
\nabla^{2} \Psi(\mathbf{p}, t)=\frac{1}{c^{2}} \frac{\partial^{2}}{\partial t^{2}} \Psi(\mathbf{p}, t)
$$

where $\Psi(\mathbf{p}, t)$ is the scalar time-dependent velocity potential related to the timedependent particle velocity by

$$
\mathbf{V}(\mathbf{p}, t)=\nabla \Psi(\mathbf{p}, t)
$$

and $c$ is the propagation velocity ( $\mathbf{p}$ and $t$ are the spatial and time variables). The time-dependent sound pressure $Q(\mathbf{p}, t)$ is given in terms of the velocity potential by

$$
Q(\mathbf{p}, t)=-\rho \frac{\partial \Psi}{\partial t}(\mathbf{p}, t)
$$

where $\rho$ is the density of the acoustic medium.

Only periodic solutions to the wave equation are considered, thus the time-dependent velocity potential $\Psi(\mathbf{p}, t)$ can be reduced to a sum of components each of the form

$$
\psi(\mathbf{p}, t)=\operatorname{Re} \varphi(\mathbf{p}) e^{-i \omega t}
$$

where $\omega$ is the angular frequency ( $\omega=2 \pi \nu$, where $\nu$ is the frequency in hertz) and $\varphi(\mathbf{p})$ is the (time-independent) velocity potential. The substitution of expression (1.14) into (1.11) reduces it to the Helmholtz (reduced wave) equation:

$$
\nabla^{2} \varphi(\mathbf{p})+k^{2} \varphi(\mathbf{p})=0
$$

where $k^{2}=\frac{\omega^{2}}{c^{2}}$ and $k$ is the wavenumber. It follows that the wavenumber and the frequency of an acoustic medium are connected by the equation

$$
k=\frac{2 \pi \nu}{c} .
$$

In order to carry out a complete solution, the wave equation is written as a series of Helmholtz problems, through expressing the boundary conditions as a Fourier series with components of the form (1.14). For each wavenumber and its associated boundary condition, the Helmholtz equation is then solved. The time-dependent sound pressure $\psi(\mathbf{p}, t)$ can then be constituted from the separate solutions. In practical situations, such as that considered in the example of the analysis of engine noise in 
Section 5.7, the wave equation is resolved into a large series of the order of hundreds or thousands of frequency components.

The sound pressure $p(\mathbf{p})$ at the point $\mathbf{p}$ in the acoustic domain is related to the velocity potential by the formula

$$
p(\mathbf{p})=i \rho \omega \varphi(\mathbf{p}) .
$$

Often sound levels are measured on the decibel scale. The magnitude in decibels of the sound pressure can be found by the expression

$$
\log _{10}\left(\left|\frac{p(\mathbf{p})}{p^{*}}\right|\right) * 20
$$

where $p^{*}$ is the reference pressure which is taken to be $2.0 \times 10^{-5} \mathrm{~Pa}$.

The phase of the signal is also important. The phase is equivalent to the angle about the origin that is made by plotting the point corresponding to the sound pressure in the Argand plane and it can be found using the arctan or $\tan ^{-1}$ function. In Fortran the generalised arctangent function ATAN2 is most useful for this and the phase is given by an expression of the form

$$
\text { ATAN2(AIMAG }(p), \operatorname{DBLE}(p)),
$$

which will return a result in the range $[0,2 \pi]$. The expression (1.19) gives the phase in radians. The phase in degrees can be found by multiplying this by $180 / \pi$.

\subsubsection{Other Acoustic Properties (Exterior Problems)}

At any frequency of enquiry, the sound pressure at all points in the acoustic domain describe the solution. However other acoustic properties are often of interest in practical situations. For example the acoustic intensity on the surface is

$$
I(\mathbf{p})=\frac{1}{2} \mathbf{R e}\left(p^{*}(\mathbf{p}) v(\mathbf{p})\right)
$$

at each point $\mathbf{p}$, where the ${ }^{*}$ denotes the complex conjugate.

The sound power is given by

$$
W=\int_{S} I(\mathbf{q}) d S_{q}
$$

or equivalently by

$$
W=\frac{1}{2 \rho c} \int_{S_{F}} p^{*}(\mathbf{q}) p(\mathbf{q}) d S_{q}
$$


where $S_{F}$ is a hypothetical closed surface in the far-field that encloses $S$.

The radiation ratio is given by

$$
\sigma_{\mathrm{RAD}}=\frac{W}{\frac{1}{2} \rho c \int_{S} v^{*}(\mathbf{q}) v(\mathbf{q}) d S_{q}} .
$$

\subsubsection{Acoustic Domains and Conditions}

In this text a Chapter is devoted to each of three distinct types of acoustic problems: the interior problem, the exterior problem and the interior modal analysis problem. The three distinct cases of two-, three- and axisymmetric three-dimensional problems are covered within each Chapter. The solution of interior and exterior boundary value problems is determined by the boundary condition and the shape of the boundary. Some classes of problem, for example the scattering problem, also involve an incident acoustic field.

The boundary condition specifies either the velocity potential $\varphi$ (that is related closely to the sound pressure by (1.17)) or the normal velocity $v$ (the derivative of $\varphi$ with respect to the normal to the boundary) or some relationship between them at the boundary points. For the boundary value problems, the boundary condition takes the following form:

$$
\alpha(\mathbf{p}) \varphi(\mathbf{p})+\beta(\mathbf{p}) v(\mathbf{p})=f(\mathbf{p}) \quad(\mathbf{p} \in S)
$$

where $\alpha, \beta$ and $f$ are complex-valued functions defined on $S$. For the modal analysis problem the boundary condition is the homogeneous form of (1.24).

In a pure radiation problem, for example, $v$ will be specified on the boundary and there will be no incident field. In a pure scattering problem the boundary condition may be specified such that it is perfectly reflecting $(v=0)$, for example, or that it has an impedance boundary condition of the form $\varphi=\gamma v$ where $\gamma$ is a constant. The general form of the boundary condition (1.24) allows different forms of condition to be specified at different regions on the boundary.

\subsubsection{Condition at Infinity for Exterior Problems}

For the exterior problems it is necessary to introduce a condition at infinity. This ensures the physical requirement that all scattered and radiated waves are outgoing. This is termed the Sommerfeld radiation condition:

$$
\lim _{r \rightarrow \infty} r^{\frac{1}{2}}\left(\frac{\partial \varphi(\mathbf{p})}{\partial r}-i k \varphi(\mathbf{p})\right)=0 \quad \text { in two dimensions and }
$$




$$
\lim _{r \rightarrow \infty} r\left(\frac{\partial \varphi(\mathbf{p})}{\partial r}-i k \varphi(\mathbf{p})\right)=0 \quad \text { in three dimensions, }
$$

where $r$ is the distance from a fixed origin to a general field point.

\subsubsection{Resonant Frequencies and Mode Shapes}

An enclosed volume of fluid exhibits resonances in the same way as a structure does. At the acoustic resonances any excitation generally leads to a (theoretically) infinite response. In practical situations the resonance frequencies inform us of the frequencies at which the acoustic response appears to be significantly magnified.

Knowledge of the modal properties can be of great value. For example a particular car design may be such that that its structural resonant frequencies coincide with the acoustic resonances of the car interior, having the apparent effect of magnifying the noise. Re-designing the car so that the two sets of resonant frequencies do not coincide could significantly reduce the interior car noise.

In acoustic problems the eigenvalues of the Helmholtz equation correspond to the resonant frequencies and the corresponding eigenfunctions to the mode shapes. They are the wavenumbers $k^{*}$ and the mode shapes $\varphi^{*}$ that satisfy the Helmholtz equation

$$
\nabla^{2} \varphi^{*}(\mathbf{p})+\left(k^{*}\right)^{2} \varphi^{*}(\mathbf{p})=0 \quad(\mathbf{p} \in D)
$$

subject to a homogeneous boundary condition of the form

$$
\alpha(\mathbf{p}) \varphi(\mathbf{p})+\beta(\mathbf{p}) v(\mathbf{p})=0 \quad(\mathbf{p} \in S)
$$

The solutions of the eigenvalue problem are related to the shape of the domain and the nature of the boundary condition. The numerical determination of the resonant frequencies and mode shapes of an enclosed fluid are considered in Chapter 6.

\subsubsection{Units of Measurement}

The units of the acoustic properties considered in this text are not always stated. Table 1.B shows the standard units that generally apply. In the case of the velocity, the velocity potential and the sound pressure, the quantities are complex-valued. These are phasar quantities; their complex value expresses both their magnitude and relative phase. 


\begin{tabular}{|l|l|}
\hline \multicolumn{2}{|c|}{ Table 1.B: Standard Units } \\
\hline Property & Unit \\
\hline mass & $\mathrm{kg}$ \\
distance & $\mathrm{m}$ \\
density & $\mathrm{kg} / \mathrm{m}^{3}$ \\
sound pressure & $\mathrm{Pa}=\mathrm{kg} / \mathrm{m}^{2}$ \\
velocity & $\mathrm{m} / \mathrm{s}$ \\
velocity potential & $\mathrm{no}$ units \\
power & $\mathrm{W}$ \\
intensity & $\mathrm{W} / \mathrm{m}^{2}$ \\
frequency & $\mathrm{Hz}$ \\
wavenumber & $\mathrm{no} \mathrm{units}$ \\
\hline
\end{tabular}

\subsubsection{Acoustic Media: Air and Water}

In linear acoustics, different acoustic media are simply reflected in the change in scaling factors that relate quantities such as wavenumber and frequency. The scaling factors can be found from tabulated values of the density $\rho$ of the fluid and the speed at which sound propagates through the fluid $c$. Note also the density of the fluid generally varies with other conditions such as the temperature of the medium.

The two most important acoustic media are those of air and water. Typically air at $20^{\circ}$ celcius and one atmosphere has a density of $1.29 \mathrm{~kg} / \mathrm{m}^{3}$ and speed of sound of $334 \mathrm{~m} / \mathrm{s}$. Water at $4^{\circ}$ celcius has a density of $1000 \mathrm{~kg} / \mathrm{m}^{3}$ and the speed of sound is $1524 \mathrm{~m} / \mathrm{s}$. From Section 1.3, equation (1.16) it can be deduced that $\nu=53.2 k$ for air and $\nu=242.6 k$ for water under the stated conditions. From equation (1.17) it can be deduced that $p=i 431 k \varphi$ for air and $p=i 1524 \times 10^{3} k \varphi$ for water under same conditions.

\subsection{Computer Programming}

The available computer software is as important in this work as the development of the boundary element method given in the text. The important subroutines are given in Table 1.A and the directory structure of the files on the relevant diskettes and on the web site is given in Appendix 1. The computer programs are written in standard Fortran 77 and they enable the reader to experience the methods in use. The source code may be observed so that the way in which the methods are implemented is clear, which is of particular value if the reader intends to develop the methods further. The 
nine major subroutines that solve each of the acoustic problems in each domain are easy to use and hence they may be employed to solve acoustic problems of particular interest to the user.

\subsubsection{Fortran}

Though the term Fortran is used freely in this text, Fortran is not a unique or static language. It has evolved over a number of decades and a number of important standards have arisen. Fortran 77 is the most important comtemporary standard but recently it has been superceded by Fortran 90 and Fortran 95. However care has been taken to ensure that later versions of Fortran are supersets of Fortran 77: programs written in Fortran 77 should also compile with a Fortran 90 compiler. Hence the software described in this text is satisfactory for either compiler.

The name Fortran is an abbreviation of formula translation: it is a language that has been pricipally written for re-creating mathematics on computer. Unlike Algol-like languages such as Pascal, Fortran code is generally not elegant and Fortran lacks the inherent brevity of $\mathrm{C}$ coding. However Fortran is still popular since it has important constructs that are dear to mathematicians, scientists and engineers - for example a complex numeric type and a good range of mathematical functions. Because of Fortran's early dominance in mathematical programming, a range of library codes for carrying out sophisticated numerical algorithms or the evaluation of special functions have been developed. Fortran software libraries such as NAG [63], IMSL [32] and reference [70] contain the results of such endeavours.

\subsubsection{Extending the work}

The source codes of the main subroutines are provided so that the user can easily extend the work covered in this text. Most simply, the test programs can be adjusted to change the description of the boundaries, the boundary conditions, the acoustic frequency, and so on so that the user can test the software further or directly apply the software to practical problems.

The subroutines for computing the interior and exterior boundary-value problems (AIBEM* and AEBEM*) can be used in an alternative way. If the parameter .LSOL. is set .FALSE. the workspace parameters WKSPC1, WKSPC2, WKSPC3, WKSPC4 contain the information required to solve a wider range of acoustic problems outside the routine. The use of the subroutines in this way is described in Appendix 7. 
At a more advanced level, the user may wish to introduce more sophistication and adjust the parameters of $\mathrm{H} 2 \mathrm{LC}, \mathrm{H} 3 \mathrm{LC}$ and $\mathrm{H} 3 \mathrm{ALC}$ accordingly in order to analyse the methods or to improve computational efficiency of the boundary element method. The core routines can be used to introduce the integral equation methods to a much wider range of acoustic problems than those explicitly covered in this text.

\subsubsection{Subordinate routines}

The major subroutines described in Chapters 3-6 require back-up modules for carrying out routine (though not necessarily simple) tasks. The following backing-software is required.

(i) A method for solving a linear system of equations where the matrices are full and complex. The subroutine CGLS for carrying this out is described in Appendix 3.

(ii) A method for finding the eigenvalues of an interpolating complex polynomial matrix is implemented by subroutine INTEIG. INTEIG requires a method for solving a generalised eigenvalue problem, again with complex matrices is implemented by subroutine CGEIG. These routines are outlined in Appendix 4.

(iii) A subroutine for computing a spherical hankel function is listed in Appendix 5.

The method (i) is required for all interior and exterior boundary value problems, the methods (ii) are only required for modal analyses and the method (iii) is only necessary for two-dimensional problems.

The method for solving the linear system of equations, necessary in the solution of the interior and exterior boundary value problems of Chapters 4 and 5, is carried out by a subroutine identified by CGLS. The modal analysis problems considered in Chapter 6 each need to call a subroutine identified by INTEIG, for solving a polynomial eigenvalue problem. This is then re-cast as a generalised eigenvalue problem (CGEIG) and solved by using a standard library routine. The hankel functions are evaluated through calling a subroutine identified by FNHANK.

In the software provided, NAG routines are invoked for solving the generalised eigenvalue problem (CGEIG) and for computing the terms of the Hankel functions (FNHANK) in the supplied software. If the reader does not have NAG then these codes will need to be developed in some alternative way. The codes for solving the interior and exterior three-dimensional boundary-values problems do not invoke any external routines. 


\subsubsection{Computational Efficiency}

Often, the greater the computer time and memory available the more accurate the result of a computational method. In practice, computer facilities are limited and the programmer will seek to trade off accuracy to reduce execution times and keep within the hardware limits of the available computer. The term computational efficiency refers to the accuracy obtained in a numerical method in relation to the work done. The greater the computational efficiency of a numerical method the better.

One of the main reasons for using the boundary element method in the first place is that it is often expected to be more computationally efficient than competing methods. For example the finite element method might be rejected as a feasible solution method for exterior acoustic problems because it is awkward to use in an infinite domain. However, the feasibility of the FEM can be equally questioned on the grounds of its poor computational efficiency in this case.

When running the boundary element method on small scale problems, solutions will usually be obtained in a few minutes or even in a matter of seconds. However, if the user is interested in developing boundary element methods for large-scale problems the issue of computational efficiency could be important; adapting the code with a view to reducing computational cost could greatly reduce the execution time in an application without a significant effect on accuracy. Alternatively, the numerical analysis of integral equation methods should ideally include a consideration of computational efficiency.

In Section 3.8 the computational cost of the core routines is tabulated. These can be used to determine the computing time required to compute the matrices in the boundary element method for example. The characteristics of the extra computational cost of running the methods for solving linear systems of equations and eigenvalue problems are documented in numerical analysis literature. The cost of function evaluations and in particular the evaluation of the Hankel functions can be determined experimentally. Hence the execution times of programs can be estimated in terms of number of elements, number of quadrature points etc. The contents of the parameter list of the core routines could be adjusted as a means to reducing the execution time. 


\section{Chapter 2}

\section{Boundary Representation}

The boundary element method can be a versatile method only if it includes the ability to represent any boundary in the given class of two-, three- or axisymmetric three-dimensional space. In general this is carried out by the facility of defining the surface as a set of panels, each having the same characteristic form (or a set of two or three characteristic forms in more advanced software). For example a closed twodimensional boundary can be represented by a set of straight lines, as illustrated earlier in Figure 1.2.

\subsection{Subdivision of the Boundary into Panels}

Let $S$ be the original boundary and $\Delta \tilde{S}_{j}$ ( for $j=1,2, . ., n$ ) be the panels that represent an approximation to $S$ in the boundary element method. If $\tilde{S}=\sum_{j=1}^{n} \Delta \tilde{S}$ is the surface described by the complete set of panels then $\tilde{S}$ is the approximation to $S$ (that is $\tilde{S} \approx S$ ). The representation of the boundary in this way is the first step in the discretisation of the integral operators that occur in the boundary integral formulation of the Helmholtz equation. Since every panel (in each particular dimensional space) has a similar characteristic form, the integration over each panel can be generalised. This function is carried out by the subroutines H2LC, H3LC and H3ALC for the two-, three- and axisymmetric three dimensional problems respectively and these subroutines form the core modules for each of the interior, exterior and modal acoustic problems. A full description of the methods employed in the discretisation of the operators by these subroutines is given in Chapter 3 .

The representation of the boundary by a set of characteristic panels enables us to easily define the boundary using a data structure. For example an ordered list of 
the coordinates of the vertices of the approximating polygon in Figure 1.2 defines the boundary. As an illustration, the boundaries of the test problems are explicitly stated in this Chapter.
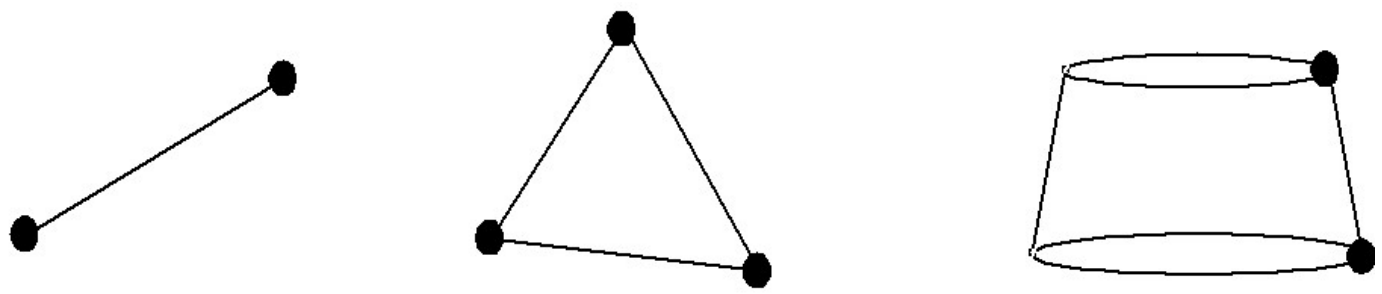

Fig 2.1. The straight line, planar triangle and truncated cone panels.

Part of the numerical error in the boundary element solution will be a result of the approximation of the boundary. A better boundary approximation and a smaller numerical error will generally arise if the boundary is represented by curved panels. The methods described in this text apply to only the simplest panels, straight line panels for two-dimensional boundaries, planar triangles for general three-dimensional boundaries and truncated conical panels for axisymmetric problems, as shown in Figure 2.1. In this Chapter we consider the representation of the boundary $S$ in terms of these panels in each dimensional space. The boundary can be expressed by two data structures in each case; the first enumerating the vertices and storing their coordinates, the second lists the individual panel by indicating the two or three vertices that define each panel.

\subsection{Two dimensional Boundaries}

In the subroutines that solve acoustic problems in two dimensions (AIBEM2, AEBEM2 and MBEM2) the boundaries must be represented in the form illustrated in Figure 1.2. In order that the normal to the boundary points outward rather than inward the two nodes that define each element must be listed in the clockwise direction around the boundary. The programs AIBEM2_T, AEBEM2_T and MBEM2_T each solve acoustic problems in which the boundary under consideration is that of a square of side 0.1 . The boundary is represented by 32 uniform panels and also has 32 vertices, as illustrated in Figure 2.2. 


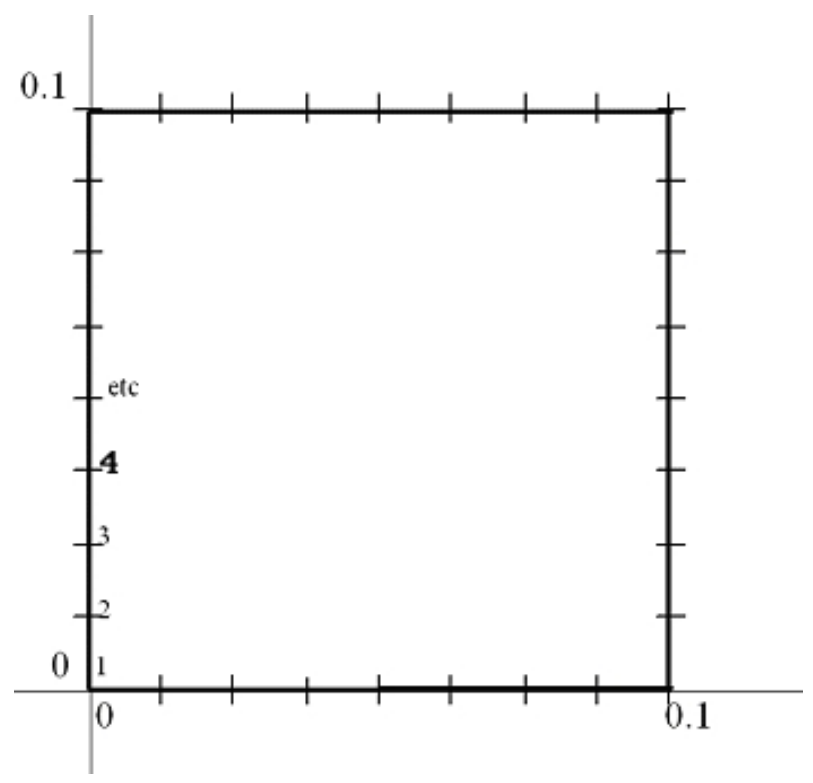

Fig 2.2. The square divided into 32 panels.

In this example the representation of the boundary is exact. In order to pass the description of the boundary to the subroutines it is defined by the two Tables of data 2.A and 2.B. Table 2.A lists the $(x, y)$ coordinates of the vertices and is identified by the real array VERTEX. Table 2.B lists the index of the two vertices that define each panel and is referred to by the integer array SELV. 


\begin{tabular}{|c|c|c||c|c|c|}
\hline \multicolumn{5}{|c|}{ Table 2.A: Vertices of square boundary (VERTEX) } \\
\hline Index & $\mathrm{x}$ & $\mathrm{y}$ & Index & $\mathrm{x}$ & $\mathrm{y}$ \\
\hline 1 & 0.0000 & 0.0000 & 17 & 0.1000 & 0.1000 \\
2 & 0.0000 & 0.0125 & 18 & 0.1000 & 0.0875 \\
3 & 0.0000 & 0.0250 & 19 & 0.1000 & 0.0750 \\
4 & 0.0000 & 0.0375 & 20 & 0.1000 & 0.0625 \\
5 & 0.0000 & 0.0500 & 21 & 0.1000 & 0.0500 \\
6 & 0.0000 & 0.0625 & 22 & 0.1000 & 0.0375 \\
7 & 0.0000 & 0.0750 & 23 & 0.1000 & 0.0250 \\
8 & 0.0000 & 0.0875 & 24 & 0.0000 & 0.0125 \\
9 & 0.0000 & 0.1000 & 25 & 0.1000 & 0.0000 \\
10 & 0.0125 & 0.1000 & 26 & 0.0875 & 0.0000 \\
11 & 0.0250 & 0.1000 & 27 & 0.0750 & 0.0000 \\
12 & 0.0375 & 0.1000 & 28 & 0.0625 & 0.0000 \\
13 & 0.0500 & 0.1000 & 29 & 0.0500 & 0.0000 \\
14 & 0.0625 & 0.1000 & 30 & 0.0375 & 0.0000 \\
15 & 0.0750 & 0.1000 & 31 & 0.0250 & 0.0000 \\
16 & 0.0875 & 0.1000 & 32 & 0.0125 & 0.0000 \\
\hline
\end{tabular}

\begin{tabular}{|c|c|c||c|c|c|}
\hline \multicolumn{5}{|c|}{ Table 2.B: Panels that constitute the square (SELV)) } \\
\hline Index & Vertex 1 & Vertex 2 & Index & Vertex 1 & Vertex 2 \\
\hline 1 & 1 & 2 & 17 & 17 & 18 \\
2 & 2 & 3 & 18 & 18 & 19 \\
3 & 3 & 4 & 19 & 19 & 20 \\
4 & 4 & 5 & 20 & 20 & 21 \\
5 & 5 & 6 & 21 & 21 & 22 \\
6 & 6 & 7 & 22 & 22 & 23 \\
7 & 7 & 8 & 23 & 23 & 24 \\
8 & 8 & 9 & 24 & 24 & 25 \\
9 & 9 & 10 & 25 & 25 & 26 \\
10 & 10 & 11 & 26 & 26 & 27 \\
11 & 11 & 12 & 27 & 27 & 28 \\
12 & 12 & 13 & 28 & 28 & 29 \\
13 & 13 & 14 & 29 & 29 & 30 \\
14 & 14 & 15 & 30 & 30 & 31 \\
15 & 15 & 16 & 31 & 31 & 32 \\
16 & 16 & 17 & 32 & 32 & 1 \\
\hline
\end{tabular}




\subsection{Three dimensional Surfaces}

In the subroutines that solve acoustic problems in three dimensions (AIBEM3, AEBEM3 and MBEM3) the boundaries must be represented in the form of a set of planar triangles. In order that the normal to the boundary points outward rather than inward the three nodes that define each element must be listed in the anti-clockwise direction when it is viewed from just outside the surface. The programs AIBEM3_T, AEBEM3_T and MBEM3_T each solve acoustic problems in which the boundary under consideration is that of a sphere of unit radius. The boundary is represented by 36 planar triangles and has 20 vertices. The approximate surface is illustrated in Figure 2.3. In this example the representation of the boundary is quite a severe approximation on the original boundary and hence some corresponding loss of accuracy should be expected in the BEM solution.

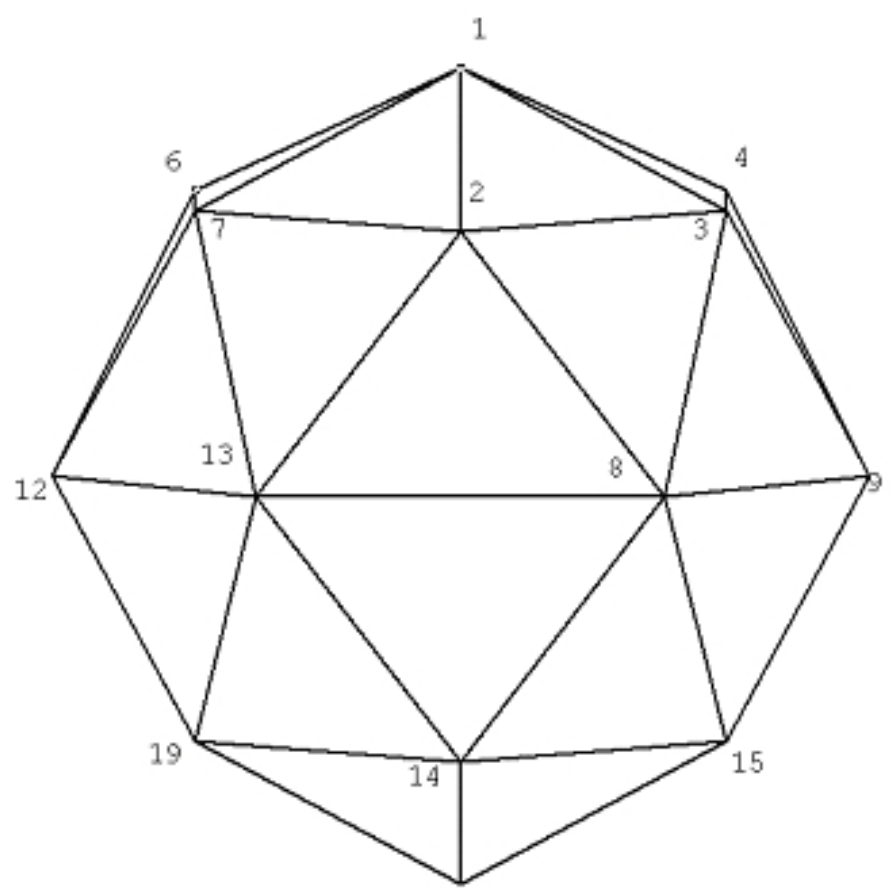

Fig 2.3. Representation of the sphere by flat triangular panels.

In order to pass the description of the boundary to the subroutines it is represented by the two tables data 2.C and 2.D. Table 2.C lists the $(x, y, z)$ coordinates of the vertices and is identified by the real array VERTEX. Table 2.D lists the index of the three vertices that define each panel and is identified by the integer array SELV. 


\begin{tabular}{|c|c|c|c||c|c|c|c|}
\hline \multicolumn{8}{|c|}{ Table 2.C: Vertices of approximate sphere boundary (VERTEX) } \\
\hline Index & $\mathrm{x}$ & $\mathrm{y}$ & $\mathrm{z}$ & Index & $\mathrm{x}$ & $\mathrm{y}$ & $\mathrm{z}$ \\
\hline 1 & 0.000 & 0.000 & 1.000 & 11 & -0.500 & -0.866 & 0.000 \\
2 & 0.000 & 0.745 & 0.667 & 12 & -1.000 & 0.000 & 0.000 \\
3 & 0.645 & 0.372 & 0.667 & 13 & -0.500 & 0.866 & 0.000 \\
4 & 0.645 & -0.372 & 0.667 & 14 & 0.000 & 0.745 & -0.667 \\
5 & 0.000 & -0.745 & 0.667 & 15 & 0.645 & 0.372 & -0.667 \\
6 & -0.645 & -0.372 & 0.667 & 16 & 0.645 & -0.372 & -0.667 \\
7 & -0.645 & 0.372 & 0.667 & 17 & 0.000 & -0.745 & -0.667 \\
8 & 0.500 & 0.866 & 0.000 & 18 & -0.645 & -0.372 & -0.667 \\
9 & 1.000 & 0.000 & 0.000 & 18 & -0.645 & 0.372 & -0.667 \\
10 & 0.500 & -0.866 & 0.000 & 20 & 0.000 & 0.000 & 1.000 \\
\hline
\end{tabular}

\begin{tabular}{|c|c|c|c||c|c|c|c|}
\hline \multicolumn{7}{|c|}{ Table 2.D: Panels that constitute the sphere (SELV)) } \\
\hline Index & Vertex 1 & Vertex 2 & Vertex 3 & Index & Vertex 1 & Vertex 2 & Vertex 3 \\
\hline 1 & 1 & 3 & 2 & 18 & 8 & 15 & 14 \\
2 & 1 & 4 & 3 & 19 & 8 & 9 & 15 \\
3 & 1 & 5 & 4 & 20 & 9 & 16 & 15 \\
4 & 1 & 6 & 5 & 22 & 9 & 10 & 16 \\
5 & 1 & 7 & 6 & 23 & 10 & 17 & 16 \\
6 & 1 & 2 & 7 & 24 & 10 & 11 & 17 \\
7 & 2 & 3 & 8 & 25 & 11 & 18 & 17 \\
8 & 3 & 9 & 8 & 26 & 11 & 12 & 18 \\
9 & 3 & 4 & 9 & 27 & 12 & 19 & 18 \\
10 & 4 & 10 & 9 & 28 & 12 & 13 & 19 \\
11 & 4 & 5 & 10 & 29 & 13 & 14 & 19 \\
12 & 5 & 11 & 10 & 30 & 13 & 8 & 14 \\
13 & 5 & 6 & 11 & 31 & 14 & 15 & 20 \\
14 & 6 & 12 & 11 & 32 & 15 & 16 & 20 \\
15 & 6 & 7 & 12 & 33 & 16 & 17 & 20 \\
16 & 7 & 13 & 12 & 34 & 17 & 18 & 20 \\
17 & 7 & 2 & 13 & 35 & 18 & 19 & 20 \\
18 & 2 & 8 & 13 & 36 & 19 & 14 & 20 \\
\hline
\end{tabular}

\subsection{Axisymmetric Surfaces}

In the subroutines that solve axisymmetric acoustic problems (AIBEMA, AEBEMA and MBEMA) the boundaries must be represented in the form of a set of truncated cone shells. In axisymmetric problems the surface can be defined by specifying the points 
on the generator and sweeping through $2 \pi$. In order that the normal to the boundary points outward rather than inward the two nodes that define each element must be listed in the clockwise direction around the generator of the boundary. The programs AIBEMA_T, AEBEMA_T and MBEMA_T each solve acoustic problems in which the boundary under consideration is that of a sphere of unit radius. The boundary is represented by 18 truncated cone shells and has 19 vertices. The approximate surface is described in Section 2.4.

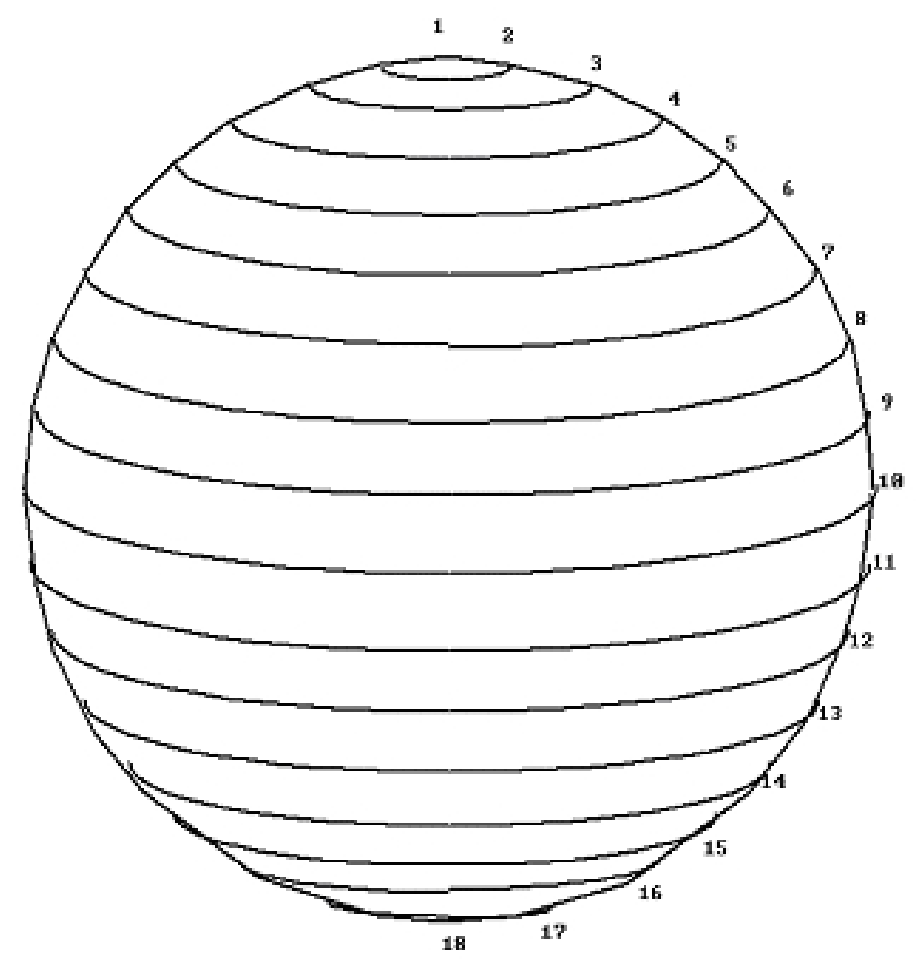

Fig 2.4. Representation of the sphere by truncated cone shells.

In order to pass the description of the boundary to the subroutines it is represented by the two tables of data 2.E and 2.F. Table 2.E lists the $(r, z)$ coordinates of the vertices and is identified by the real array VERTEX. Table 2.F lists the index of the two vertices that define each panel and is identified by the integer array SELV. 


\begin{tabular}{|c|c|c||c|c|c|}
\hline \multicolumn{6}{|c|}{ Table 2.E: Vertices of sphere generator (VERTEX) } \\
\hline Index & $\mathrm{r}$ & $\mathrm{z}$ & Index & $\mathrm{r}$ & $\mathrm{z}$ \\
\hline 1 & 0.000 & 1.000 & 11 & 0.985 & -0.174 \\
2 & 0.174 & 0.985 & 12 & 0.940 & -0.342 \\
3 & 0.342 & 0.940 & 13 & 0.866 & -0.500 \\
4 & 0.500 & 0.866 & 14 & 0.766 & -0.643 \\
5 & 0.643 & 0.766 & 15 & 0.643 & -0.766 \\
6 & 0.766 & 0.643 & 16 & 0.500 & -0.866 \\
7 & 0.866 & 0.500 & 17 & 0.342 & -0.940 \\
8 & 0.940 & 0.342 & 18 & 0.174 & -0.985 \\
9 & 0.985 & 0.174 & 19 & 0.000 & -1.000 \\
10 & 1.000 & 0.000 & & & \\
\hline
\end{tabular}

\begin{tabular}{|c|c|c||c|c|c|}
\hline \multicolumn{5}{|c|}{ Table 2.F: Panels that constitute the sphere (SELV)) } \\
\hline Index & Vertex 1 & Vertex 2 & Index & Vertex 1 & Vertex 2 \\
\hline 1 & 1 & 2 & 10 & 10 & 11 \\
2 & 2 & 3 & 11 & 11 & 12 \\
3 & 3 & 4 & 12 & 12 & 13 \\
4 & 4 & 5 & 13 & 13 & 14 \\
5 & 5 & 6 & 14 & 14 & 15 \\
6 & 6 & 7 & 15 & 15 & 16 \\
7 & 7 & 8 & 16 & 16 & 17 \\
8 & 8 & 9 & 17 & 17 & 18 \\
9 & 9 & 10 & 18 & 18 & 19 \\
\hline
\end{tabular}

\subsection{Conclusion}

In this Chapter it has been shown how boundaries in each dimensional setting can be represented by two data structures. The square and the sphere represented by triangles and axisymmetric cone panel are used throughout the remainder of this text to demonstrate the boundary element methods. In the subroutines of Chapters 4, 5 and 6 the two data structures that represent the relevant structure are passed as array parameters. By setting the validation parameter LVALID=.TRUE. in the subroutines a check is made to ensure that the boundaries are closed.

The subdivision of the square and the sphere into triangles enables us to simulate general acoustic problems in two- and three- dimensions. However, the axisymmetric elements are uniform when rotated about the $z$-axis. Conical elements should only be used when the acoustic field as well as the surface is known to be axisymmetric. 
In the 2D example of the square in Figure 2.2, the panels are of uniform length. For the sphere illustrated in Figure 2.3 the triangles are of approximately equal size. As a general rule, the element sizes for general two- and three-dimensional boundaries should be as close to uniform as possible across the boundary. Moreover, in the threedimensional case, the triangles should not deviate too far from the equilateral shape. For axisymmetric surfaces, the lengths of the generator of the elements should be as close to uniform size as possible. If the validation parameter is set LVALID $=$.TRUE. then the input panels are checked to ensure that their sizes are reasonably uniform and the input boundary is checked to ensure it does not contain sharp angles.

In some cases it is wise to overrule the general guidelines of the previous paragraph. For example if a boundary has an intricate shape in a localised area it would be beneficial to use more elements in the area so that the boundary is satisfactorily represented. In other cases it may be known that the potential is strongly varying in some areas of the surface - for example in the neighbourhood of a sharp corner the potential can be singular - and in these regions it is often beneficial to increase the number of elements.

The approximation methods used in this Chapter are very simple, but each is sufficient to approximate the boundaries in each class of domain. For more intricate geometries, the boundary would be better represented by curved panels. Unfortunately, curved elements require careful computational treatment and such elements are not considered in this text. In the next Chapter the elements are completed by also including a representation of the boundary functions. 


\section{Chapter 3}

\section{The Discrete Helmholtz Operators}

This Chapter considers the general problem of constructing methods for discretising the integral operators, as required in the application of collocation to integral equation formulations of the Helmholtz equation. The Chapter is important only if the precise algorithms used to compute the discrete operators need to be known or the reader wishes to develop programs for solving acoustic problems that are not covered in the text. Readers wishing to go directly to the solution of interior, exterior or modal analysis problems may omit this Chapter.

Linear acoustic or Helmholtz problems obviously give rise to a range of integral equation formulations, for example depending on whether the acoustic field lies in an interior or exterior domain. However, the integral equations that arise in all problems in the same spatial dimensions contain similar integral operators. For example a computational method for evaluating the discrete from of the integral operators in a three-dimensional exterior acoustic problem can also be used in any other threedimensional acoustic problem.

For each dimensional space it is possible to develop a module for computing the discrete form of the integral operators that is common to the interior, exterior and modal analysis subroutines. The purpose of this Section is to show how the discrete forms of the integral operators in the three spatial dimensions are computed and to introduce the subroutines $\mathrm{H} 2 \mathrm{LC}, \mathrm{H} 3 \mathrm{LC}$ and $\mathrm{H} 3 \mathrm{ALC}$ that have been developed in order to carry this out [33]. The naming of the subroutines is such that the $\mathrm{H}$ represents Helmholtz, the 2, 3, 3A identifies the dimensionality, the LC represents the linear boundary approximation with a constant function approximation on each panel. 


\subsection{The Helmholtz Integral Operators}

The subroutines compute the discrete form of the integral operators $L_{k}, M_{k}, M_{k}^{t}$ and $N_{k}$ that arise in the application of collocation to integral equation formulations of the Helmholtz equation. Expressions for the discrete integral operators are derived by approximating the boundaries by the most simple panels for each of the three cases - straight line panels for the general two-dimensional case, flat triangular panels for the general three-dimensional case and conical panels for the axisymmetric threedimensional case - and approximating the boundary functions by a constant on each panel. For each particular case of boundary division, the discrete form of the operators is computed using the subroutines H2LC (two-dimensional), H3LC (three-dimensional) and H3ALC (axisymmetric three-dimensional).

\subsubsection{The Helmholtz Operators}

The Helmholtz integral operators are defined as follows:

$$
\begin{gathered}
\left\{L_{k} \zeta\right\}_{\Gamma}(\mathbf{p}) \equiv \int_{\Gamma} G_{k}(\mathbf{p}, \mathbf{q}) \zeta(\mathbf{q}) d S_{q} \\
\left\{M_{k} \zeta\right\}_{\Gamma}(\mathbf{p}) \equiv \int_{\Gamma} \frac{\partial G_{k}}{\partial n_{q}}(\mathbf{p}, \mathbf{q}) \zeta(\mathbf{q}) d S_{q} \\
\left\{M_{k}^{t} \zeta\right\}_{\Gamma}\left(\mathbf{p} ; \mathbf{u}_{p}\right) \equiv \frac{\partial}{\partial u_{p}} \int_{\Gamma} G_{k}(\mathbf{p}, \mathbf{q}) \zeta(\mathbf{q}) d S_{q} \\
\left\{N_{k} \zeta\right\}_{\Gamma}\left(\mathbf{p} ; \mathbf{u}_{p}\right) \equiv \frac{\partial}{\partial u_{p}} \int_{\Gamma} \frac{\partial G_{k}}{\partial n_{q}}(\mathbf{p}, \mathbf{q}) \zeta(\mathbf{q}) d S_{q}
\end{gathered}
$$

where $\Gamma$ is a boundary or partial boundary, $\mathbf{n}_{q}, \mathbf{u}_{p}$ are unit vectors with $\mathbf{n}_{q}$ the unique normal to $\Gamma$ at $\mathbf{q}$ and $\zeta(\mathbf{q})$ is a function defined for $\mathbf{q} \in \Gamma . G_{k}(\mathbf{p}, \mathbf{q})$ is the free-space Green's function for the Helmholtz equation.

\subsubsection{Green's functions}

Let the Green's functions be denoted by $G_{k}$ and they are defined as follows:

$$
\begin{gathered}
G_{k}(\mathbf{p}, \mathbf{q})=\frac{i}{4} H_{0}^{(1)}(k r) \quad(k \in \mathrm{C} \backslash\{0\}) \text { in two dimensions, } \\
G_{k}(\mathbf{p}, \mathbf{q})=\frac{1}{4 \pi} \frac{e^{i k r}}{r}(k \in \mathrm{C}) \text { in three dimensions, }
\end{gathered}
$$


where $r=|\mathbf{r}|, \mathbf{r}=\mathbf{p}-\mathbf{q}, \mathrm{C}$ is the set of complex numbers and $i$ is the unit imaginary number. The function $H_{0}^{(1)}$ is the spherical Hankel function of the first kind of order zero. The Green's functions (3.5) and (3.6) also satisfy the Sommerfeld radiation condition for $|k|>0$.

For the special case when $k=0$ the Helmholtz equation (1.15) is the Laplace equation. In this particular case the chosen Green's functions will be

$$
\begin{gathered}
G_{0}(\mathbf{p}, \mathbf{q})=-\frac{1}{2 \pi} \log r \quad \text { in two dimensions }, \\
G_{0}(\mathbf{p}, \mathbf{q})=\frac{1}{4 \pi} \frac{1}{r} \quad \text { in three dimensions. }
\end{gathered}
$$

Note that $\lim _{k \rightarrow 0} G_{k}(\mathbf{p}, \mathbf{q})=G_{0}(\mathbf{p}, \mathbf{q})$ for the three-dimensional case but not for the two dimensional case and that $G_{0}(\mathbf{p}, \mathbf{q})$ for the two-dimensional case does not satisfy condition (1.25).

\subsubsection{Properties of the Operators}

In general for a given function $\zeta(\mathbf{p})(\mathbf{p} \in S),\left\{L_{k} \zeta\right\}_{\Gamma}(\mathbf{p})$ and $\left\{N_{k} \zeta\right\}_{\Gamma}\left(\mathbf{p} ; \mathbf{u}_{p}\right)$ are continuous across the boundary $\Gamma$ (for any given unit vector $\mathbf{u}_{p}$ in the definition of the latter function). The $\left\{M_{k} \zeta\right\}_{\Gamma}(\mathbf{p})$ and $\left\{M_{k}^{t} \zeta\right\}_{\Gamma}(\mathbf{p})$ are discontinuous at $\Gamma$ and continuous on the remainder of the domain. The operators $M_{k}$ and $M_{k}^{t}$ have the following continuity properties at points in the neighbourhood of $\Gamma$ :

$$
\lim _{\epsilon \rightarrow 0}\left\{M_{k} \zeta\right\}_{\Gamma}\left(\mathbf{p}+\epsilon \mathbf{n}_{p}\right)+\frac{1}{2} \zeta(\mathbf{p})=\left\{M_{k} \zeta\right\}_{\Gamma}(\mathbf{p})=\lim _{\epsilon \rightarrow 0}\left\{M_{k} \zeta\right\}_{\Gamma}\left(\mathbf{p}-\epsilon \mathbf{n}_{p}\right)-\frac{1}{2} \zeta(\mathbf{p}),
$$

$\lim _{\epsilon \rightarrow 0}\left\{M_{k}^{t} \zeta\right\}_{\Gamma}\left(\mathbf{p}+\epsilon \mathbf{n}_{p} ; \mathbf{n}_{p}\right)-\frac{1}{2} \zeta(\mathbf{p})=\left\{M_{k}^{t} \zeta\right\}_{\Gamma}\left(\mathbf{p} ; \mathbf{n}_{p}\right)=\lim _{\epsilon \rightarrow 0}\left\{M_{k}^{t} \zeta\right\}_{\Gamma}\left(\mathbf{p}-\epsilon \mathbf{n}_{p} ; \mathbf{n}_{p}\right)+\frac{1}{2} \zeta(\mathbf{p})$, where $\mathbf{p} \in \Gamma$ and $\mathbf{n}_{p}$ is the unit normal to the $\Gamma$ at $\mathbf{p}$. The continuity properties are slightly different if $\Gamma$ is not smooth at $\mathbf{p}$.

\subsection{Some Properties of the Kernel Functions}

The results given in this Section are extracted mainly from Burton [16], [17]. In the following $\mathbf{r}=\mathbf{p}-\mathbf{q}$ and $r=|\mathbf{r}|, G_{k}=G_{k}(\mathbf{p}, \mathbf{q}), G_{0}=G_{0}(\mathbf{p}, \mathbf{q})$.

\subsubsection{Derivatives of $G_{0}$ with respect to $r$}

In two dimensions we have

$$
\frac{\partial G_{0}}{\partial r}=-\frac{1}{2 \pi} \frac{1}{r}
$$




$$
\frac{\partial^{2} G_{0}}{\partial r^{2}}=\frac{1}{2 \pi} \frac{1}{r^{2}}
$$

In three dimensions we have

$$
\begin{aligned}
& \frac{\partial G_{0}}{\partial r}=-\frac{1}{4 \pi} \frac{1}{r^{2}} \\
& \frac{\partial^{2} G_{0}}{\partial r^{2}}=\frac{1}{2 \pi} \frac{1}{r^{3}}
\end{aligned}
$$

\subsubsection{Derivatives of $G_{k}(k \neq 0)$ with respect to $r$}

In two dimensions we have

$$
\frac{\partial G_{k}}{\partial r}=-\frac{i}{4} k H_{1}^{(1)}
$$

where $H_{1}^{(1)}$ is the spherical Hankel function of the first kind and of order one and

$$
\frac{\partial^{2} G_{k}}{\partial r^{2}}=\frac{i}{4} k^{2}\left(\frac{H_{1}^{(1)}}{k r}-H_{0}^{(1)}\right) .
$$

In three dimensions we have

$$
\begin{gathered}
\frac{\partial G_{k}}{\partial r}=\frac{e^{i k r}}{4 \pi r^{2}}(i k r-1), \\
\frac{\partial^{2} G_{k}}{\partial r^{2}}=\frac{e^{i k r}}{4 \pi r^{3}}\left(2-2 i k r-k^{2} r^{2}\right) .
\end{gathered}
$$

\subsubsection{Expressions for the normal derivatives of $G_{k}$}

The following expressions hold in both two and three dimensions and for all $k$ :

$$
\begin{gathered}
\frac{\partial G_{k}}{\partial n_{q}}=\frac{\partial G_{k}}{\partial r} \frac{\partial r}{\partial n_{q}}, \\
\frac{\partial G_{k}}{\partial u_{p}}=\frac{\partial G_{k}}{\partial r} \frac{\partial r}{\partial u_{p}}, \\
\frac{\partial^{2} G_{k}}{\partial u_{p} \partial n_{q}}=\frac{\partial G_{k}}{\partial r} \frac{\partial^{2} r}{\partial u_{p} \partial n_{q}}+\frac{\partial^{2} G_{k}}{\partial r^{2}} \frac{\partial r}{\partial u_{p}} \frac{\partial r}{\partial n_{q}} .
\end{gathered}
$$

\subsubsection{Expressions for the normal derivative of $r$}

The derivatives of $r$ with respect to $\mathbf{u}_{p}$ and $\mathbf{n}_{q}$ may be written as follows:

$$
\frac{\partial r}{\partial n_{q}}=-\frac{\mathbf{r} \cdot \mathbf{n}_{q}}{r}
$$


www.boundary-element-method.com

$$
\begin{gathered}
\frac{\partial r}{\partial u_{p}}=\frac{\mathbf{r} \cdot \mathbf{n}_{p}}{r} \\
\frac{\partial^{2} r}{\partial u_{p} \partial n_{q}}=-\frac{1}{r}\left(\mathbf{u}_{p} \cdot \mathbf{n}_{q}+\frac{\partial r}{\partial u_{p}} \frac{\partial r}{\partial n_{q}}\right) .
\end{gathered}
$$

\subsubsection{Expressions for $\frac{\partial^{2} G_{0}}{\partial u_{p} \partial n_{q}}$}

The following results can be derived from the substitution of (3.22) and (3.9),(3.10) or (3.11),(3.12) into (3.19) with $k=0$ :

$$
\begin{gathered}
\frac{\partial^{2} G_{0}}{\partial u_{p} \partial n_{q}}=\frac{1}{2 \pi r^{2}}\left(\mathbf{u}_{p} \cdot \mathbf{n}_{q}+2 \frac{\partial r}{\partial u_{p}} \frac{\partial r}{\partial n_{q}}\right) \text { in two dimensions, } \\
\frac{\partial G_{0}}{\partial u_{p} \partial n_{q}}=\frac{1}{4 \pi r^{3}}\left(\mathbf{u}_{p} \cdot \mathbf{n}_{q}+3 \frac{\partial r}{\partial u_{p}} \frac{\partial r}{\partial n_{q}}\right) \text { in three dimensions. }
\end{gathered}
$$

\subsubsection{Asymptotic Properties}

In the following results, $\mathbf{p}, \mathbf{q} \in \Gamma$ where $\Gamma$ is a surface and $\Gamma$ is smooth at $\mathbf{p}$ :

$$
\begin{gathered}
\lim _{\mathbf{q} \rightarrow \mathbf{p}}\left(G_{k}(\mathbf{p}, \mathbf{q})-G_{0}(\mathbf{p}, \mathbf{q})\right)=O\left(r^{0}\right), \\
\lim _{\mathbf{q} \rightarrow \mathbf{p}} \frac{\partial G_{k}}{\partial u_{p}}(\mathbf{p}, \mathbf{q})=O\left(r^{0}\right), \\
\lim _{\mathbf{q} \rightarrow \mathbf{p}} \frac{\partial G_{k}}{\partial n_{q}}(\mathbf{p}, \mathbf{q})=O\left(r^{0}\right), \\
\lim _{\mathbf{q} \rightarrow \mathbf{p}}\left(\frac{\partial^{2} G_{k}}{\partial u_{p} \partial n_{q}}(\mathbf{p}, \mathbf{q})-\frac{\partial^{2} G_{0}}{\partial u_{p} \partial n_{q}}(\mathbf{p}, \mathbf{q})+\frac{1}{2} k^{2} G_{k}(\mathbf{p}, \mathbf{q})\right)=O\left(r^{0}\right) .
\end{gathered}
$$

\subsection{Discretisation}

In order to derive the discrete forms of the integral operators (3.1), (3.2), (3.3) and (3.4), $\Gamma$ is approximated by a set of $n$ panels $\tilde{\Gamma}=\sum_{j=1}^{n} \Delta \tilde{\Gamma}_{j}$. The boundary function $\zeta$ is replaced by its equivalent on the approximate boundary $\tilde{\Gamma}$. The function is then replaced by a constant on each panel. Thus for the $L_{k}$ integral operator:

$$
\left\{L_{k} \zeta\right\}_{\Gamma}(\mathbf{p}) \approx\left\{L_{k} \tilde{\zeta}\right\}_{\tilde{\Gamma}}(\mathbf{p}) \approx \sum_{j=1}^{n} \int_{\Delta \tilde{\Gamma}_{j}} G_{k}(\mathbf{p}, \mathbf{q}) \tilde{\zeta}\left(\mathbf{p}_{j}\right) d S_{q} \approx \sum_{j=1}^{n}\left[\tilde{\zeta}\left(\mathbf{p}_{j}\right)\left\{L_{k} \tilde{e}\right\}_{\Delta \Gamma_{j}}(\mathbf{p})\right],
$$


where $\tilde{e}$ is the unit function. The other integral operators may be discretised in a similar way.

The discrete forms are thus defined as follows:

$$
\begin{gathered}
\left\{L_{k} \tilde{e}\right\}_{\Delta \tilde{\Gamma}_{j}}(\mathbf{p})=\int_{\Delta \tilde{\Gamma}_{j}} G_{k}(\mathbf{p}, \mathbf{q}) d S_{q}, \\
\left\{M_{k} \tilde{e}\right\}_{\Delta \tilde{\Gamma}_{j}}(\mathbf{p})=\int_{\Delta \tilde{\Gamma}_{j}} \frac{\partial G_{k}}{\partial n_{q}}(\mathbf{p}, \mathbf{q}) d S_{q}, \\
\left\{M_{k}^{t} \tilde{e}\right\}_{\Delta \tilde{\Gamma}_{j}}\left(\mathbf{p} ; \mathbf{u}_{p}\right)=\frac{\partial}{\partial u_{p}} \int_{\Delta \tilde{\Gamma}_{j}} G_{k}(\mathbf{p}, \mathbf{q}) d S_{q} \text { and } \\
\left\{N_{k} \tilde{e}\right\}_{\Delta \tilde{\Gamma}_{j}}\left(\mathbf{p} ; \mathbf{u}_{p}\right)=\frac{\partial}{\partial u_{p}} \int_{\Delta \tilde{\Gamma}_{j}} \frac{\partial G_{k}}{\partial n_{q}}(\mathbf{p}, \mathbf{q}) d S_{q} .
\end{gathered}
$$

The derivative operator in (3.32) can always be carried inside the integral. The same is true for the operator in (3.33) when $\mathbf{p}$ does not lie on the panel $\Delta \tilde{\Gamma}_{j}$. Thus we may write:

$$
\begin{gathered}
\left\{M_{k}^{t} \tilde{e}\right\}_{\Delta \tilde{\Gamma}_{j}}\left(\mathbf{p} ; \mathbf{u}_{p}\right)=\int_{\Delta \tilde{\Gamma}_{j}} \frac{\partial G_{k}}{\partial u_{p}}(\mathbf{p}, \mathbf{q}) d S_{q} \\
\left\{N_{k} \tilde{e}\right\}_{\Delta \tilde{\Gamma}_{j}}\left(\mathbf{p} ; \mathbf{u}_{p}\right)=\int_{\Delta \tilde{\Gamma}_{j}} \frac{\partial^{2} G_{k}}{\partial u_{p} \partial n_{q}}(\mathbf{p}, \mathbf{q}) d S_{q} \text { when } \mathbf{p} \notin \Delta \tilde{\Gamma}_{j}
\end{gathered}
$$

When $\mathbf{p} \notin \Delta \tilde{\Gamma}_{j}$ the integrals of (3.30), (3.31), (3.32) and (3.33) will all be regular and hence are amenable to standard quadrature. The same is true for the integrands of (3.31) and (3.32) when $\mathbf{p} \in \Delta \tilde{\Gamma}_{j}$ (although not on the edge of the panel). However, the evaluation of the discrete integral operators (3.30) and (3.33) generally require special treatment when $\mathbf{p} \in \Delta \tilde{\Gamma}_{j}$.

The special techniques applied here involve 'subtracting out' the singularity and evaluating the singular part and remaining regular part separately. The following results are immediate from the asymptotic properties of the kernel functions (3.25) and $(3.28)$ :

$$
\begin{gathered}
\left\{L_{k} \tilde{e}\right\}_{\Delta \tilde{\Gamma}_{j}}(\mathbf{p})=\left\{L_{0} \tilde{e}\right\}_{\Delta \tilde{\Gamma}_{j}}(\mathbf{p})+\int_{\Delta \tilde{\Gamma}_{j}}\left(G_{k}(\mathbf{p}, \mathbf{q})-G_{0}(\mathbf{p}, \mathbf{q})\right) d S_{q} \\
\left\{N_{k} \tilde{e}\right\}_{\Delta \tilde{\Gamma}_{j}}\left(\mathbf{p} ; \mathbf{u}_{p}\right)=\left\{N_{0} \tilde{e}\right\}_{\Delta \tilde{\Gamma}_{j}}\left(\mathbf{p} ; \mathbf{u}_{p}\right)-\frac{1}{2} k^{2}\left\{L_{0} \tilde{e}\right\}_{\Delta \tilde{\Gamma}_{j}}(\mathbf{p})+ \\
\int_{\Delta \tilde{\Gamma}_{j}}\left(\frac{\partial^{2} G_{k}}{\partial u_{p} \partial n_{q}}(\mathbf{p}, \mathbf{q})-\frac{\partial^{2} G_{0}}{\partial u_{p} \partial n_{q}}(\mathbf{p}, \mathbf{q})+\frac{1}{2} k^{2} G_{0}(\mathbf{p}, \mathbf{q})\right) d S_{q}
\end{gathered}
$$

where in each of (3.36) and (3.37) the explicit integral is non-singular. Evaluation in this way requires the computation of the regular integral (amenable to standard quadrature) and the determination of the subtracted out part. 
In summary, the evaluation of the integral operators requires a summation of a set of integrand values multiplied by quadrature weights. In the case when $\mathbf{p} \in \Delta \tilde{\Gamma}_{j}$ the evaluation of the subtracted out part is also required for the $L_{k}$ and $N_{k}$ operators.

\subsection{Evaluation of the Discrete Forms}

In this Section a list of operations for computing the discrete integral operators is given. This particular programme of computation is given prominence because it needs to be executed for each quadrature point and hence it is the key to determining the computational cost of the overall method. The following computational programme is intended to be optimal. Note that it is assumed that $\mathbf{p}$ and $\mathbf{u}_{p}$ are already set.

A: Set $\mathbf{q}$ the point on the panel

B: Set $\mathbf{n}_{q}$ the unit outward normal to the panel at $\mathbf{q}$

C: Compute $\mathbf{u}_{p} \cdot \mathbf{n}_{q}$

D: Compute $\mathbf{r}$

E: Compute $r, r^{2}$

F: Compute $r^{3}$ (in the 3D case)

G: Compute $\frac{\partial r}{\partial n_{q}}$ via (3.20)

H: Compute $\frac{\partial r}{\partial u_{p}}$ via (3.21)

I: Compute $\frac{\partial r}{\partial n_{q}} * \frac{\partial r}{\partial u_{p}}$

J: Compute $\frac{\partial^{2} r}{\partial u_{p} \partial n_{q}}$ via (3.22)

$\mathrm{K}$ : Compute $k r[k * r]$ and $i k r[i * k r]$

L: Compute $s k r[k r * k r]$ in the 3D case)

M: Compute $H$ [Hankel function $\left.H_{0}^{(1)}(k r), H_{1}^{(1)}(k r)\right]$ for 2D problems or $E[\exp (i k r)$ ] for 3D problems.

N: Compute Green's function via (3.5) or (3.7) for 2D or via (3.6) or (3.8) for 3D.

O: Multiply $L_{k}$ kernel by weight and add to sum

P: Compute $\frac{\partial G_{k}}{\partial r}$ via (3.13) or (3.9) for 2D or via (3.15) or (3.11) for 3D

Q: Compute value of quadrature weight multiplied by the result of operation $\mathrm{N}$.

R: Compute $M_{k}$ kernel multiplied by weight and add to sum

S: Compute $M_{k}^{t}$ kernel multiplied by weight and add to sum

T: Compute $\frac{\partial^{2} G_{k}}{\partial r^{2}}$ via (3.14) or (3.10) for 2D or via (3.16) or (3.12) for 3D

$\mathrm{U}$ : Multiply $N_{k}$ kernel by weight and add to sum 
Apart from operations $\mathrm{E}$ and $\mathrm{M}$, each operation can be directly costed in terms of floating-point operations. Operation E computes a square root and operation $\mathrm{M}$ computes a Hankel function in two-dimensional problems and a complex exponential in three-dimensional problems. The square root and the complex exponential functions are available in most programming languages, although in some cases it could be beneficial (in terms of computational cost) not to use the standard language functions.

In the two-dimensional case, operation $M$ requires the computation of the spherical Hankel functions or $\log$ functions when $k$ is zero. Since Hankel functions are not generally available as standard functions, then some external routine is required for their evaluation.

In the subroutines H2LC, H3LC and H3ALC the square root function and the exponential and/or Hankel function or log functions that are evaluated at each quadrature point need to be provided as external functions with the identifiers FNSQRT, FNEXP, FNHANK and FNLOG. The freedom to define the functions externally and to choose the quadrature rule allows the user to take full control of the efficiency of the subroutines.

\subsection{General Introduction to the Subroutines}

The subroutines have the identifiers H2LC, H3LC and H3ALC, for computing the discrete Helmholtz integral operators for the two-dimensional, three-dimensional and axisymmetric cases. The subroutines' parameter list have the following general form:

\footnotetext{
SUBROUTINE $\mathrm{H}\{2$ or 3 or $\mathrm{A}\} \mathrm{LC}($

complex wavenumber,

point ( $\mathbf{p}$ and the unit vector $\mathbf{u}_{p}$, if necessary),

geometry of the panel (vertices which define panel),

quadrature rule (weights and abscissae for the standard element),

validation and control parameters,

discrete Helmholtz integral operators (solution),

work space ).

View files H2LC.FOR, H3LC.FOR and H3ALC.FOR [33] in order to observe the subroutines.
} 


\subsubsection{General control of the subroutines}

The parameter complex $\mathrm{K}$ passes the wavenumber $k$ to the subroutines. More efficient methods are applied in the subroutines if the value of $\mathrm{K}$ is zero, real or imaginary. These special cases of the wavenumber are discerned through testing whether the size of the real part or imaginary part is less than or greater than the parameter EK.

The logical variable LVALID enables the user to switch on (.TRUE.) or off (.FALSE.) the validation of the input parameters. Setting LVALID=.TRUE. puts a series of tests on the input data into effect. The use of this facility will have a computational cost but could be useful when initially constructing a program that calls the subroutines or to facilitate the diagnosis of an error that appears in the calling program or in the subroutine.

The logical variables LLK, LMK, LMKT and LNK allows the user to choose the specific operators of $L_{k}, M_{k}, M_{k}^{t}$ or $N_{k}$ that are required. The LLK, LMK, LMKT and LNK that have .TRUE. values results in the values of the corresponding discrete operator to appear in DISLK, DISMK, DISMKT and DISNK respectively, on exit from the subroutine.

\subsubsection{Geometrical Information}

Real one-dimensional Fortran arrays define the point $\mathbf{p}$ and the geometry of the panel; they have two components in H2LC and H3ALC and three components in H3LC. In H2LC and H3LC, the components of the arrays define the Cartesian coordinates of the points and the Cartesian components of the directional vector $\mathbf{u}_{p}$. In H3ALC, the components of the array define the cylindrical polar coordinates of the points and the cylindrical polar components of $\mathbf{u}_{p}$.

Following from the techniques of defining the surfaces in Chapter 2, in H2LC and H3ALC two points QA and QB, each one-dimensional real arrays in Fortran with two components, define the panel. In H2LC, QA and QB are the edges of the straight line panel. In H3ALC, QA and QB define the outer rims of the truncated conical shell panel, the two points lying on the edges of the generator of the panel. In H3LC, three points QA, QB and QC, each one-dimensional real arrays in Fortran with three components, are the vertices that define the planar triangular panel.

The normal to the panel is computed within the subroutines. In H2LC and H3ALC, the normal is defined to be $[\mathrm{QA}(2)-\mathrm{QB}(2), \mathrm{QB}(1)-\mathrm{QA}(1)]$ normalised. Hence the normal is to the right on the line $\mathrm{QA}-\mathrm{QB}$. Note that in $\mathrm{H} 3 \mathrm{ALC}$ the normal is the normal 
on the generator of the panel. In H3LC the normal is defined to be [QB-QA] $\times$ [QC-QA] (normalised), where $\times$ denotes the vector cross product. Hence the normal is presumed to be outward (to the observer) when QA-QB-QC are arranged anticlockwise around the triangle.

The integrals that define $L_{k}$ and $N_{k}$ require special treatment when the point $\mathbf{p}$ lies on the panel. The logical variable LPONEL is used to inform the subroutine as to whether the point $\mathbf{p}$ lies on the panel (.TRUE.) or not (.FALSE.). In the case LPONEL=.TRUE., methods based on the expressions for the integral operators in equations (3.36) and (3.37) are used.

For the computation of the discrete $M_{k}^{t}$ and $N_{k}$ operators the vector $\mathbf{u}_{p}$ needs to be defined. In the subroutines it is denoted VECP and it must represent a unit vector. Results for a non-unit vector $\mathbf{u}_{p}$ can be obtained by scaling the vector $\mathbf{u}_{p}$ before calling the subroutine (so that it is a unit vector) and suitably scaling the results DISMKT and DISNK afterwards. If the point $\mathbf{p}(\mathrm{P})$ lies on the panel then $\mathbf{u}_{p}$ (VECP) must be the unit normal to the panel, as defined above.

\subsubsection{Quadrature Rule}

In subroutine $\mathrm{H} 2 \mathrm{LC}$ a quadrature rule (a set of weights and abscissae) over the unit interval must be input. A quadrature rule over the standard triangle is required in H3LC and a quadrature rule over the unit square (consisting of standard quadrature rules applied in both the generator and angular directions) must be input to H3ALC. The input abscissae are mapped onto the panel over which the integration is carried out. In general, the greater the number of quadrature points, and the more efficient the choice of quadrature rule, the more accurate the resulting discrete integral operators will be. However, the time taken for the execution of each subroutine is proportional to the number of quadrature points (with an overhead cost).

When the point $\mathbf{p}$ does not lie on the panel, the integrand is continuously differentiable and hence standard quadrature rules are satisfactory. However, when $\mathbf{p}$ lies on the panel, the integrands are continuous (after the subtractions described by equations (39) and (40)), but they are generally not differentiable at the point $\mathbf{p}$. If the input quadrature rule does not take this property into account then it could lead to an inaccurate evaluation of the discrete forms.

In a practical computation, one of the subroutines will be called many times. The subroutines allow different quadrature rules to be input for each call. For example when $\mathbf{p}$ lies on the panel a special quadrature rule is advised, as discussed above. 
Also if one panel is relatively large or relatively close to the point $\mathbf{p}$ then a relatively more accurate quadrature rule is generally desirable.

In subroutines $\mathrm{H} 2 \mathrm{LC}$ and $\mathrm{H} 3 \mathrm{LC}$ the parameter MAXNQ sets the limit on the number of quadrature points in the quadrature rule defined in the generator and in the angular direction. The values of these parameters must not be changed between calls of the subroutines. The actual number of quadrature points, denoted NQ, must be less than or equal to MAXNQ but can be altered between calls. For the H3ALC subroutine the number of quadrature points consists of NGQ along the generator and NTQ in the angular direction. The values of NGQ and NTQ may be changed between calls of H3ALC, but they must always be less than MAXNGQ and MAXNTQ respectively. Hence the total number of quadrature points used in $H 3 A L C$ is $N G Q \times N T Q$.

In subroutine $\mathrm{H} 2 \mathrm{LC}$, the parameters $A Q$ and $W Q$ store the abscissae and the weights of the quadrature rule. The parameters AGQ, WGQ and ATQ, WTQ store similar quadrature rules for the generator and angular directions in H3ALC. Note that in $\mathrm{H} 3 \mathrm{ALC}$ the panel is defined as the result of rotating the generator QA-QB about the $z$ axis. In practice, the size of the panel in the angular direction will vary greatly between calls of H3ALC. Since it is good practice to distribute the quadrature points fairly evenly over the panel, the number of quadrature points should be used in the angular direction should be roughly proportional to the 'radius' of the panel.

Generally the most efficient quadrature rules to use in subroutines H2LC and H3ALC are standard Gauss-Legendre rules. The weights and abscissae for such rules are listed in Stroud and Secrest [79] and can also be generated using NAG routine D01BBF, for example. When the point $\mathbf{p}$ lies on the panel then an efficient quadrature rule is obtainable by dividing the domain at $\mathbf{p}$ and using suitably scaled Gauss-Legendre rules on the separate regions. Note that such division is only necessary in the generator direction in $\mathrm{H} 3 \mathrm{ALC}$.

In subroutine $\mathrm{H} 3 \mathrm{LC}$, the parameters $\mathrm{XQ}, \mathrm{YQ}, \mathrm{WQ}$ store the $x$ coordinates, the $y$ coordinates and the weights of the quadrature rule over the standard triangle. Quadrature rules of the Gauss-Legendre type are listed in Laursen and Gellert [56]. In the case when $\mathbf{p}$ lies on the panel then a suitable quadrature rule can be obtained by dividing the triangle into three regions, by connecting the point $\mathbf{p}$ to the vertices of the panel, and map suitably scaled standard quadrature rules onto the three separate triangular regions. 


\subsubsection{Validation of the input}

If LVALID is .TRUE. then a file for storing and error messages or warnings must be open prior to calling the subroutine. For subroutine H2LC the file H2LC.ERR must be opened using a Fortran statement like OPEN(UNIT=10,FILE='H2LC.ERR'). Similarly, for subroutines H3LC and H3ALC the files H3LC.ERR and H3ALC.ERR respectively should be opened.

If LVALID is .TRUE. then the parameters to the subroutines EGEOM and EQRULE set the maximum absolute tolerance allowed in the input geometrical and quadrature rule data. For example if the components of QA and QB are all within EGEOM of each other (the points coincide) or the sum of the weights in the quadrature rules is greater than $N Q \times E Q R U L E$ (allowing for an error of EQRULE in each component) then an error message will be output to the error file. In such cases the subroutine will be aborted and the parameter LFAIL will register .TRUE..

\subsection{Subroutines}

\subsubsection{Subroutine $\mathrm{H} 2 \mathrm{LC}$}

Subroutine H2LC computes the values of (3.30)-(3.33) for straight line elements with chosen parameters $k(\mathrm{~K}), \mathbf{p}(\mathrm{P}), \mathbf{u}_{p}(\mathrm{VECP}), \Delta \tilde{\Gamma}_{j}(\mathrm{QA}$ and $\mathrm{QB})$.

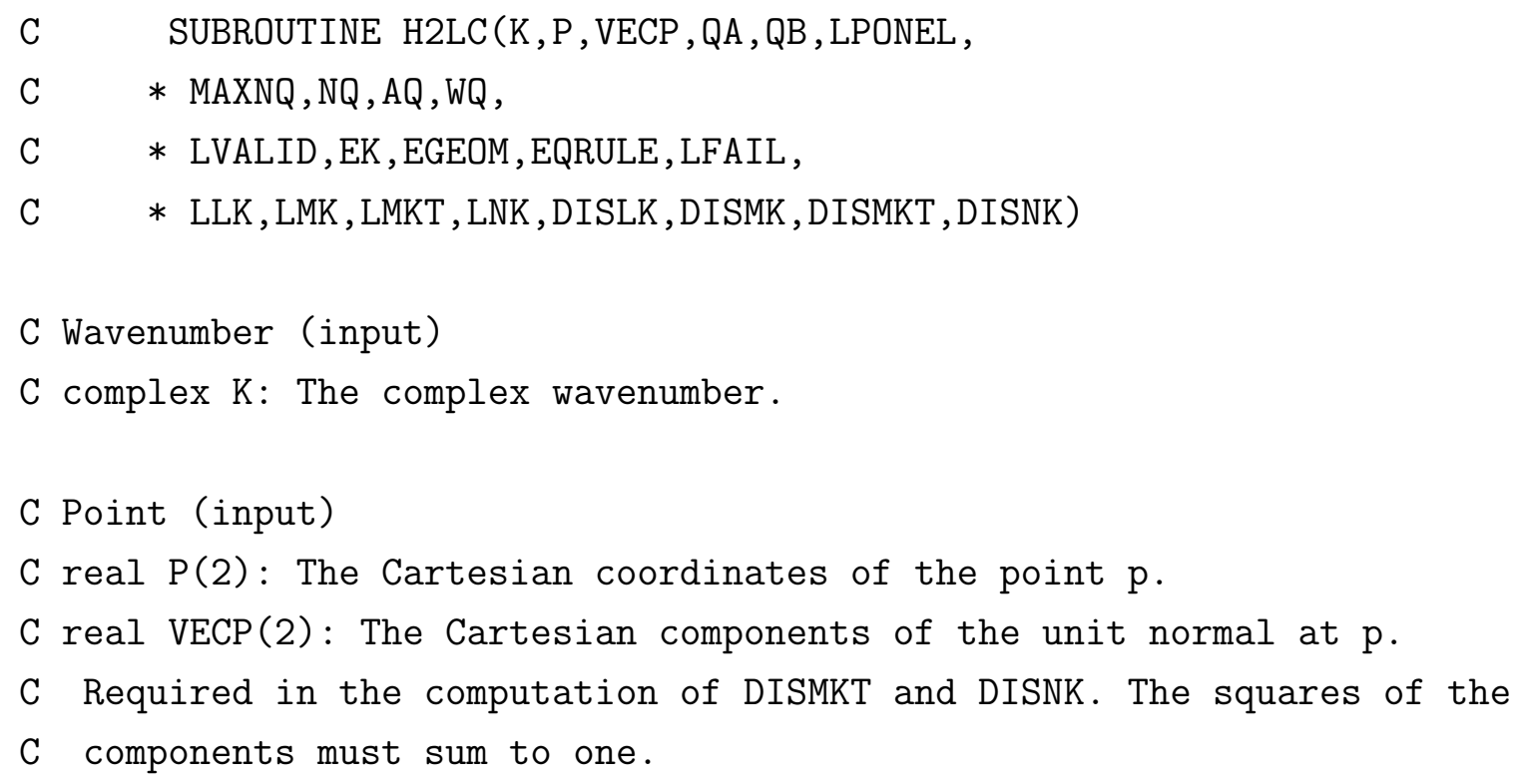


C Geometry of element (input)

C real QA(2): The Cartesian coordinates of the first edge of the

C element.

C real QB(2): The Cartesian coordinates of the second edge of the

C element.

C logical LPONEL: If the point $P(2)$ lies on QA-QB then LPONEL must be

$C$ set .TRUE., otherwise LPONEL must be set .FALSE. .

C Quadrature rule (input)

$C$ integer MAXNQ: The limit on the size of the quadrature rule. The

$C$ value should not be changed between calls of H2LC. MAXNQ $>=1$.

$C$ integer NQ: The actual number of quadrature rule points. $1=<N Q<=$ MAXNQ.

C real AQ(MAXNQ): The quadrature rule abscissae. The values must lie in

$\mathrm{C}$ the domain $[0,1]$ and be in ascending order.

C real WQ(MAXNQ): The quadrature rule weights which correspond to the

C quadrature points in AQ. The components of WQ must sum to one.

C Validation and control parameters (input)

C logical LVALID: A switch to enable choice of checking of subroutine

C parameters (.TRUE.) or not (.FALSE.).

$C$ real EK: The maximum absolute error expected in $\mathrm{K}$. This is used

$C$ to classify $K$ as 'zero', 'real', 'imaginary' or 'complex'.

C real EGEOM: The maximum absolute error in the parameters that

$\mathrm{C}$ describe the geometry. Value is of importance only when

C LVALID $=$.TRUE. .

C real EQRULE: The maximum absolute error in the components of the

C quadrature rule data. Value is of importance only when LVALID=.TRUE. .

C Validation parameter (output)

C logical LFAIL: Value is only important if LVALID=.TRUE.. If

C LFAIL=.FALSE. then the input data has been found to be satisfactory.

C If LFAIL=.TRUE. then the input data has been found to be

C unsatisfactory. The subroutine would have been aborted. The output

C parameters DISLK, DISMK, DISMKT and DISNK will all be zero. A

C diagnosis will be given in the file H2LC.ERR.

C Choice of discrete forms required (input) 
C logical LLK: If discrete form of Lk operator is required then set C .TRUE., otherwise set .FALSE. .

C logical LMK: If discrete form of Mk operator is required then set C .TRUE., otherwise set .FALSE. .

C logical LMKT: If discrete form of Mkt operator is required then set C .TRUE., otherwise set .FALSE. .

C logical LNK: If discrete form of Nk operator is required then set C .TRUE., otherwise set .FALSE. .

C Discrete Helmholtz integral operators (output)

C complex DISLK: The discrete Lk integral operator.

C complex DISMK: The discrete Mk integral operator.

C complex DISMKT: The discrete Mkt integral operator.

C complex DISNK: The discrete $\mathrm{Nk}$ integral operator.

\subsubsection{Subroutine H3LC}

Subroutine H3LC computes the values of (3.30)-(3.33) for planar triangular elements with chosen parameters $k(\mathrm{~K}), \mathbf{p}(\mathrm{P}), \mathbf{u}_{p}(\mathrm{VECP}), \Delta \tilde{\Gamma}_{j}(\mathrm{QA}, \mathrm{QB}$ and $\mathrm{QC})$.

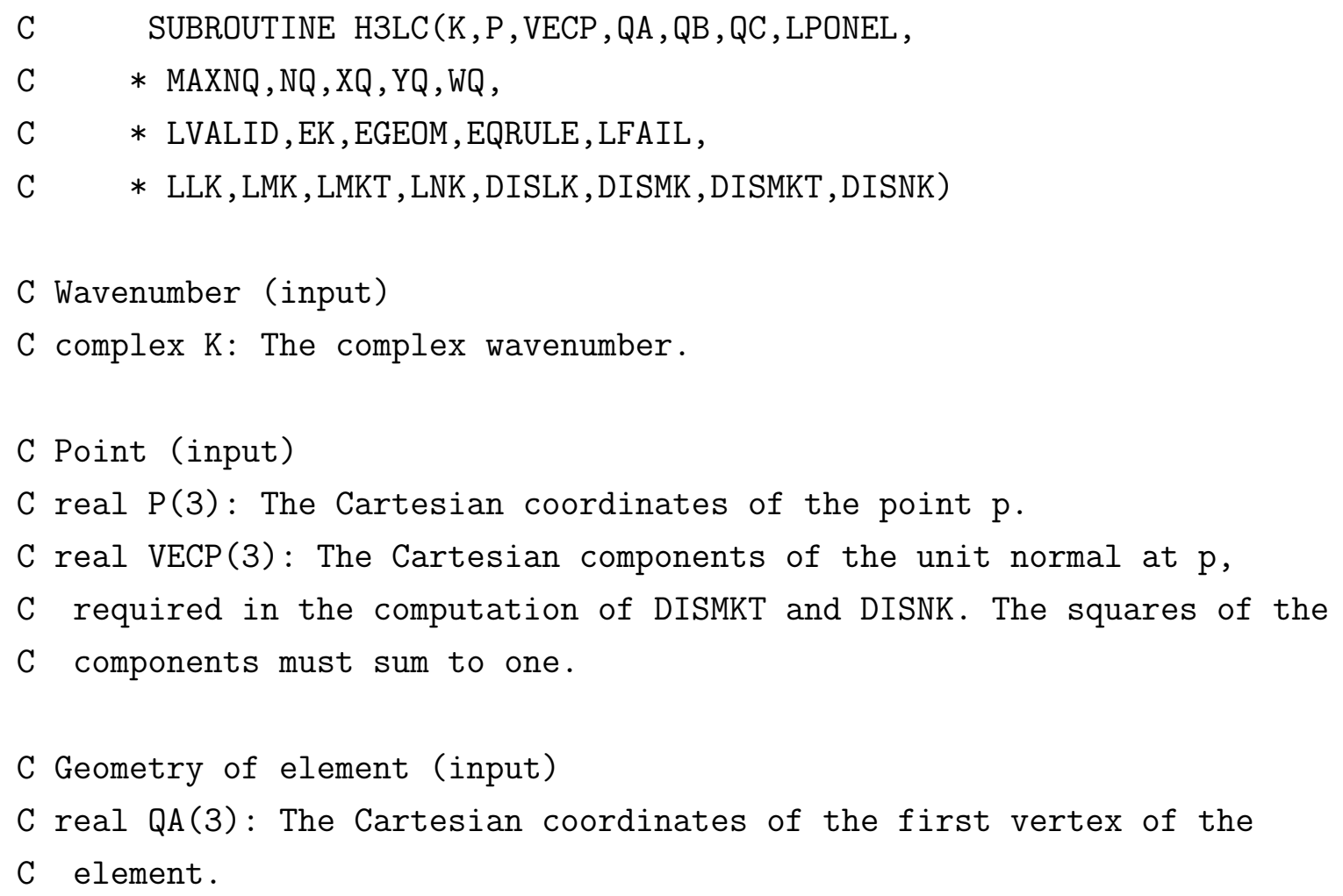


C real QB(3): The Cartesian coordinates of the second vertex of the

C element.

C real QC(3): The Cartesian coordinates of the third vertex of the

C element.

C logical LPONEL: If the point $\mathrm{P}(3)$ lies on element QA-QB-QC then LPONEL

C must be set .TRUE., otherwise LPONEL must be set .FALSE..

C Quadrature rule (input)

$C$ integer MAXNQ: The limit on the size of the quadrature rule. The value

C should not be changed between calls of H3LC. MAXNQ $>=1$.

C integer NQ: The actual number of quadrature rule points. $1<=N Q<=M A X N Q$.

C real XQ(MAXNQ): The $\mathrm{X}$-coordinate of the quadrature rule abscissae. The C values must lie in the standard triangle.

C real YQ(MAXNQ): The $y$-coordinate of the quadrature rule abscissae. The

C values must lie in the standard triangle.

C real WQ(MAXNQ): The quadrature rule weights which correspond to the

$\mathrm{C}$ quadrature points in $\mathrm{XQ}$ and $\mathrm{YQ}$. The components of WQ must sum to one.

C Validation and control parameters (input)

C logical LVALID: A switch to enable choice of checking of subroutine

C parameters (.TRUE.) or not (.FALSE.).

$C$ real EK: The maximum absolute error expected in $\mathrm{K}$. This is used

C to classify K as 'zero', 'real', 'imaginary' or 'complex'.

$C$ real EGEOM: The maximum absolute error in the parameters that

C describe the geometry. Value is of importance only when

C LVALID $=$.TRUE. .

C real EQRULE: The maximum absolute error in the components of the

C quadrature rule data. Value is of importance only when LVALID=.TRUE..

C Validation parameter (output)

C logical LFAIL: Value is only important if LVALID=.TRUE. If

C LFAIL=.FALSE. then the input data has been found to be satisfactory.

C If LFAIL=.TRUE. then the input data has been found to be

C unsatisfactory. The subroutine would have been aborted. The output

C parameters DISLK, DISMK, DISMKT and DISNK will all be zero. A

C diagnosis will be given in the file H3LC.ERR. 
C Choice of discrete forms required (input)

C logical LLK: If discrete form of Lk operator is required then set

C .TRUE., otherwise set .FALSE. .

C logical LMK: If discrete form of Mk operator is required then set C .TRUE., otherwise set .FALSE. .

C logical LMKT: If discrete form of Mkt operator is required then set

C .TRUE., otherwise set .FALSE. .

C logical LNK: If discrete form of Nk operator is required then set C .TRUE., otherwise set .FALSE. .

C Discrete Helmholtz integral operators (output)

C complex DISLK: The discrete Lk integral operator.

C complex DISMK: The discrete Mk integral operator.

C complex DISMKT: The discrete Mkt integral operator.

C complex DISNK: The discrete Nk integral operator.

\subsubsection{Subroutine H3ALC}

Subroutine H3ALC computes the values of (3.30)-(3.33) for conical elements with chosen parameters $k(\mathrm{~K}), \mathbf{p}(\mathrm{P}), \mathbf{u}_{p}(\mathrm{VECP}), \Delta \tilde{\Gamma}_{j}$ (QA and QB).

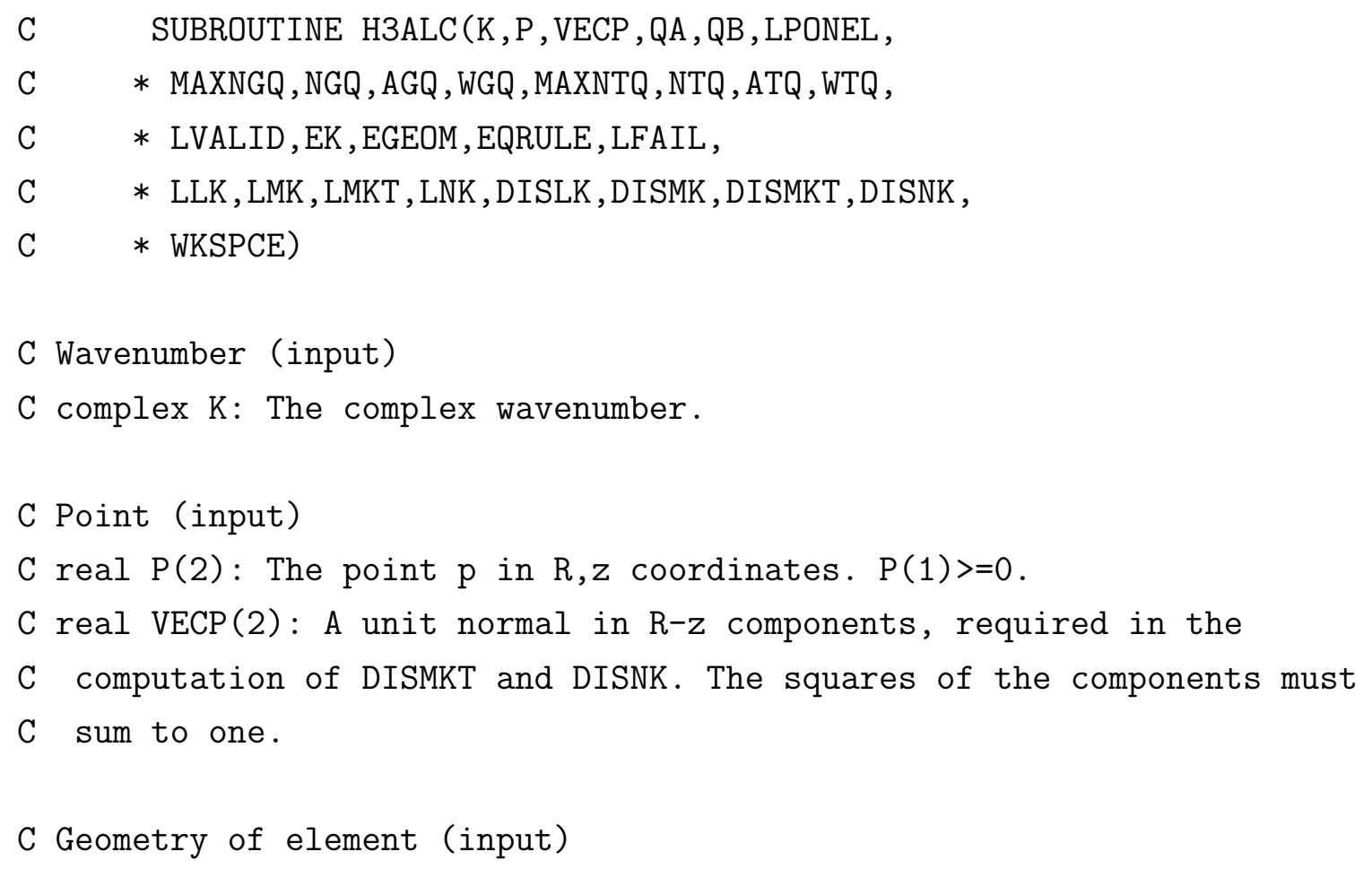


C real QA(2): The $R-z$ coordinates of the first edge of the element.

C $Q A(1)>=0$.

$C$ real $Q B(2)$ : The $R-z$ coordinates of the second edge of the element.

C $Q B(1)>=0$.

C logical LPONEL: If the point $P$ lies on QA-QB then LPONEL must be set

C .TRUE., otherwise LPONEL must be set .FALSE. .

C Generator-direction quadrature rule (input)

$C$ integer MAXNGQ: The limit on the size of the generator-direction

$C$ quadrature rule. The value should not be changed between calls of

C H3ALC. MAXNGQ $>=1$.

$C$ integer NGQ: The actual number of generator-direction quadrature

C rule points. $1<=N G Q<=M A X N G Q, N G Q<=L I M N G$.

C real AGQ(MAXNGQ): The generator-direction quadrature rule abscissae.

$C$ The values must lie in the domain $[0,1]$ and be in ascending order.

C real WGQ (MAXNGQ): The generator-direction quadrature rule weights

C which correspond to the quadrature points in AGQ. The components of

C WGQ must sum to one.

C Theta-direction quadrature rule (input)

C integer MAXNTQ: The limit on the size of the theta-direction

$C$ quadrature rule. The value should not be changed between calls of

C H3ALC. MAXNTQ $>=1$.

$C$ integer NTQ: The actual number of theta-direction quadrature rule

C points. $1<=\mathrm{NTQ}<=\mathrm{MAXNTQ}$.

C real ATQ(MAXNTQ): The theta-direction quadrature rule abscissae. The

C values must lie in the domain $[0,1]$.

C real WTQ(MAXNTQ): The theta-direction quadrature rule weights which

C correspond to the quadrature points in ATQ. The components of WTQ

C must sum to one.

C Validation and control parameters (input)

C logical LVALID: A switch to enable choice of checking of subroutine

C parameters (.TRUE.) or not (.FALSE.).

$C$ real EK: The maximum absolute error expected in $\mathrm{K}$. This is used

C to classify K as 'zero', 'real', 'imaginary' or 'complex'.

$C$ real EGEOM: The maximum absolute error in the parameters that 
C describe the geometry. Value is of importance only when

C LVALID $=$.TRUE. .

C real EQRULE: The maximum absolute error in the components of the

C quadrature rule data. Value is of importance only when LVALID=.TRUE..

C Validation parameter (output)

C logical LFAIL: Value is only important if LVALID=.TRUE. If

C LFAIL=.FALSE. then the input data has been found to be satisfactory.

C If LFAIL=.TRUE. then the input data has been found to be

C unsatisfactory. The subroutine would have been aborted. The output

C parameters DISLK, DISMK, DISMKT and DISNK will all be zero. A

C diagnosis of the errors will be given in the file H3ALC.ERR.

C Choice of discrete forms required (input)

C logical LLK: If discrete form of Lk operator is required then set

C .TRUE., otherwise set .FALSE. .

C logical LMK: If discrete form of Mk operator is required then set C .TRUE., otherwise set .FALSE. .

C logical LMKT: If discrete form of Mkt operator is required then set

C .TRUE., otherwise set .FALSE. .

C logical LNK: If discrete form of Nk operator is required then set

C .TRUE., otherwise set .FALSE. .

C Discrete Helmholtz integral operators (output)

C complex DISLK: The discrete Lk integral operator.

C complex DISMK: The discrete Mk integral operator.

C complex DISMKT: The discrete Mkt integral operator.

C complex DISNK: The discrete Nk integral operator.

C Work space

C real WKSPCE (2*MAXNTQ+MAXNGQ)

\subsection{Special Numerical Integration Methods}

Special methods are required to evaluate the singular integrals that arise in the discrete $L_{k}$ and $N_{k}$ operators when the collocation point lies on the element. 


\subsubsection{Two-dimensional problems}

The regular integrals that arise are approximated by a standard quadrature rule such as a Gauss-Legendre rule which is specified in the parameter list to the subroutines. Tables of Gauss-Legendre rules are given in Stroud and Secrest [79] and can also generated from the NAG library [63], for example. The non-regular integrals that arise in the formulae (3.36) and (3.37) are computed via the following methods. See Jaswon and Symm [34] and Kirkup [40] for the background to these methods.

The $M_{0}$ and $M_{0}^{t}$ operators have regular kernels, hence the aim is to find expressions for the following:

$$
\begin{gathered}
\left\{L_{0} \tilde{e}\right\}_{\Delta \tilde{\Gamma}}(\mathbf{p})=\int_{\Delta \tilde{\Gamma}} G_{0}(\mathbf{p}, \mathbf{q}) d S_{q} \\
\left\{N_{0} \tilde{e}\right\}_{\Delta \tilde{\Gamma}}\left(\mathbf{p} ; \mathbf{n}_{p}\right)=\frac{\partial}{\partial n_{p}} \int_{\Delta \tilde{\Gamma}} \frac{\partial G_{0}}{\partial n_{q}}(\mathbf{p}, \mathbf{q}) d S_{q},
\end{gathered}
$$

where $\Delta \tilde{\Gamma}$ is a straight line panel, $\mathbf{p} \in \Delta \tilde{\Gamma}$ (though not on an edge or corner of the panel). Let it be assumed that the panel $\Delta \tilde{\Gamma}$ has length $a+b$ with $\mathbf{q}=\mathbf{q}(x)$ and $\mathbf{p}=\mathbf{q}(0)$ for $x \in[-a, b]$. This gives the following formulae for (3.38) and (3.39):

$$
\begin{gathered}
\left\{L_{0} \tilde{e}\right\}_{\Delta \tilde{\Gamma}}(\mathbf{p})=\frac{1}{2 \pi}[a+b-a \log a-b \log b] \\
\left\{N_{0} \tilde{e}\right\}_{\Delta \tilde{\Gamma}}\left(\mathbf{p} ; \mathbf{n}_{p}\right)=-\frac{1}{2 \pi}\left[\frac{1}{a}+\frac{1}{b}\right] .
\end{gathered}
$$

\subsubsection{Three-dimensional problems}

The regular integrals that arise are approximated by a quadrature rule defined on a triangle. Laursen and Gellert [56] contains a selection of Gauss-Legendre quadrature rules for the standard triangle. The non-regular integrals that from discretising the $L_{k}$ and $N_{k}$ operators are computed by the following methods. See Jaswon and Symm [34], Terai [80], Banerjee and Butterfield [7] and Kirkup [40] for the background to these methods.

The $M_{0}$ and $M_{0}^{t}$ operators have regular kernels, hence the aim is to find expressions for:

$$
\begin{gathered}
\left\{L_{0} \tilde{e}\right\}_{\Delta \tilde{\Gamma}}(\mathbf{p})=\int_{\Delta \tilde{\Gamma}} G_{0}(\mathbf{p}, \mathbf{q}) d S_{q} \\
\left\{N_{0} \tilde{e}\right\}_{\Delta \tilde{\Gamma}}\left(\mathbf{p} ; \mathbf{n}_{p}\right)=\frac{\partial}{\partial n_{p}} \int_{\Delta \tilde{\Gamma}} \frac{\partial G_{0}}{\partial n_{q}}(\mathbf{p}, \mathbf{q}) d S_{q}
\end{gathered}
$$


where $\Delta \tilde{\Gamma}$ is a planar triangular panel, $\mathbf{p} \in \Delta \tilde{\Gamma}$ (though not on an edge or corner of the panel). Let $R(\theta)$ be the distance from $\mathbf{p}$ to the edge of the panel for $\theta \in[0,2 \pi]$, as illustrated in Figure 3.1.

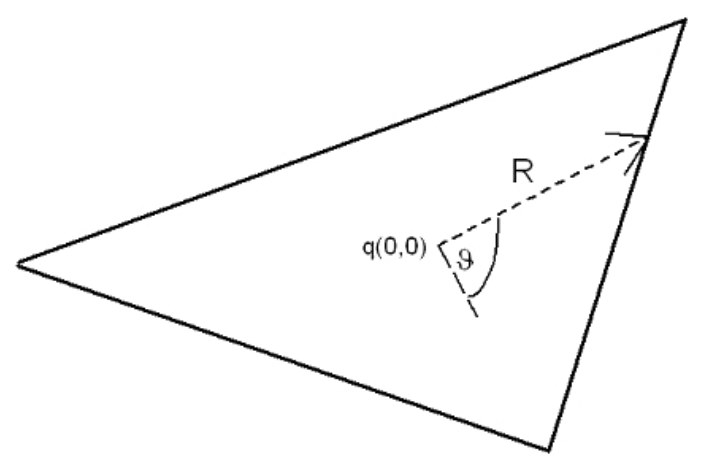

Fig. 3.1. Polar integration on the planar triangle panel.

The integrals (3.42) and (3.43) may be written in the form:

$$
\begin{gathered}
\left\{L_{0} \tilde{e}\right\}_{\Delta \tilde{\Gamma}}(\mathbf{p})=\frac{1}{4 \pi} \int_{0}^{2 \pi} R(\theta) d \theta, \\
\left\{N_{0} \tilde{e}\right\}_{\Delta \tilde{\Gamma}}\left(\mathbf{p} ; \mathbf{u}_{p}\right)=-\frac{1}{4 \pi} \int_{0}^{2 \pi} \frac{1}{R(\theta)} d \theta .
\end{gathered}
$$

In order to evaluate the integrals, the triangular panel $\Delta \tilde{\Gamma}$ is divided into three $\triangle_{1}$, $\triangle_{2}$ and $\triangle_{3}$ by joining the point $\mathbf{p}$ to the vertices. The resulting triangles have the form of Figure 3.2.

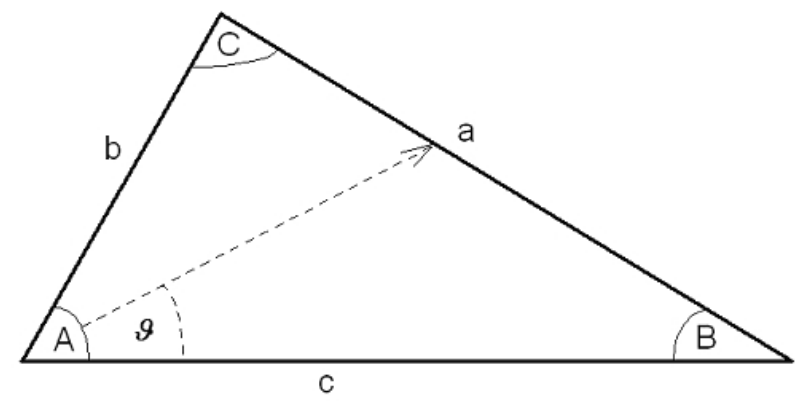

Fig 3.2. Division of the planar triangle panel.

After some elementary analysis, we obtain

$$
\begin{gathered}
\left\{L_{0} \tilde{e}\right\}_{\Delta \tilde{S}}(\mathbf{p})=\sum_{\triangle_{1}, \triangle_{2}, \triangle_{3}} \frac{1}{4 \pi} R(0) \sin B\left(\log \tan \left(\frac{B+A}{2}\right)-\log \tan \frac{B}{2}\right) \text { and } \\
\left\{N_{0} \tilde{e}\right\}_{\Delta \tilde{S}}\left(\mathbf{p} ; \mathbf{u}_{p}\right)=\sum_{\triangle_{1}, \triangle_{2}, \triangle_{3}} \frac{1}{4 \pi} \frac{\cos (B+A)-\cos B}{R(0) \sin B} .
\end{gathered}
$$




\subsubsection{Axisymmetric three-dimensional problems}

The regular integrals that arise are approximated by a two-dimensional quadrature rule defined on a rectangle which is specified in the parameter list to the subroutine. These integrals can be approximated using a Gauss-Legendre rule in the generator and $\theta$ directions. The non-regular integrals that arise in the formula are computed by the following methods.

The $M_{0}$ and $M_{0}^{t}$ operators have regular kernels, hence the aim is to find expressions for the following:

$$
\begin{gathered}
\left\{L_{0} \tilde{e}\right\}_{\Delta \tilde{\Gamma}}(\mathbf{p})=\int_{\Delta \tilde{\Gamma}} G_{0}(\mathbf{p}, \mathbf{q}) d S_{q} \\
\left\{N_{0} \tilde{e}\right\}_{\Delta \tilde{\Gamma}}\left(\mathbf{p} ; \mathbf{n}_{p}\right)=\frac{\partial}{\partial n_{p}} \int_{\Delta \tilde{\Gamma}} \frac{\partial G_{0}}{\partial n_{q}}(\mathbf{p}, \mathbf{q}) d S_{q},
\end{gathered}
$$

where $\Delta \tilde{\Gamma}$ is a conical shell panel, $\mathbf{p} \in \Delta \tilde{\Gamma}$ (though not on an edge of the panel).

The integral in (3.44) is evaluated through dividing the integral with respect to the generator direction into two parts at $\mathbf{p}$ and transforming the integral through changing the power of the variable in line with a method described in references [25] and [40]. The resulting regular integral on both parts is computed via the quadrature rule supplied to the routine.

The integral in (3.45) is evaluated by using the result that if the surface of integration in (3.45) is extended to enclose a three-dimensional volume then the integral vanishes (see [40]). As each panel is a truncated right circular cone shell a $45^{\circ}$ right circular cone is added to each flat side of the panel. The integrals over the two $45^{\circ}$ cones are regular and are computed by a composite rule based on the quadrature rule based on the quadrature rule supplied to the subroutine. The solution is thus equal to minus the sum of the integrals over the two $45^{\circ}$ cones.

\subsection{Analysis of Computational Cost}

The subroutines H2LC, H3LC and H3ALC were written with the aim of minimising the computational cost of evaluating the discrete form when the point $\mathbf{p}$ does not lie on the panel. When $\mathbf{p}$ does lie on the panel then each of the subroutines will generally be more costly, this is particularly true for H3ALC. However, in integral equation methods the cost of evaluating the discrete form when $\mathbf{p}$ does not lie on the panel is the key to estimating the overall computational cost. 


\begin{tabular}{|c|c|c|c|c|}
\hline \multicolumn{5}{|c|}{ Table 3.A: Required Operations } \\
\hline Op. & $L_{k}$ & $M_{k}$ & $M_{k}^{t}$ & $N_{k}$ \\
\hline$A$ & $x$ & $x$ & $x$ & $x$ \\
\hline B & & $x$ & & $x$ \\
\hline C & & & & $x$ \\
\hline $\mathrm{D}$ & $\times$ & $x$ & $\times$ & $\times$ \\
\hline$E$ & $\times$ & $x$ & $\times$ & $x$ \\
\hline $\mathrm{F}$ & & & & $\circ$ \\
\hline G & & $x$ & & $\times$ \\
\hline $\mathrm{H}$ & & & $\times$ & $x$ \\
\hline I & & & & $\times$ \\
\hline $\mathrm{J}$ & & & & $x$ \\
\hline K & $x$ & $x$ & $x$ & $x$ \\
\hline $\mathrm{L}$ & & & & $\times$ \\
\hline M & $\times$ & $x$ & $\times$ & $\times$ \\
\hline $\mathrm{N}$ & $\times$ & & & \\
\hline $\mathrm{O}$ & $\times$ & & & \\
\hline$P$ & & $x$ & $x$ & $x$ \\
\hline Q & & $x$ & $x$ & \\
\hline $\mathrm{R}$ & & $\times$ & $\times$ & \\
\hline $\mathrm{S}$ & & & $\times$ & \\
\hline $\mathrm{T}$ & & & & $\times$ \\
\hline$U$ & & & & $\times$ \\
\hline $\begin{array}{r}\times: \\
\circ:\end{array}$ & $\begin{array}{l}\text { Pera } \\
\text { Oper }\end{array}$ & $\begin{array}{l}\text { ion } r \\
\text { ation }\end{array}$ & $\begin{array}{l}\text { quire } \\
\text { equir }\end{array}$ & $\begin{array}{l}\mathrm{D} \text { and } 3 \\
3 \mathrm{D} \text { only }\end{array}$ \\
\hline
\end{tabular}




\begin{tabular}{|c||c|c|c|}
\hline \multicolumn{4}{|c|}{ Table 3.B: Cost of Operations A to J } \\
\hline Op. & $2 \mathrm{D}$ & $3 \mathrm{D}$ & 3 Daxi \\
\hline $\mathrm{A}$ & $2 C_{f}$ & $6 C_{f}$ & $2 C_{f}$ \\
$\mathrm{~B}$ & 0 & 0 & $2 C_{f}$ \\
$\mathrm{C}$ & 0 & 0 & $2 C_{f}$ \\
$\mathrm{D}$ & 0 & 0 & 0 \\
$\mathrm{E}$ & $C_{s}+2 C_{f}$ & $C_{s}+3 C_{f}$ & $C_{s}+3 C_{f}$ \\
$\mathrm{~F}$ & - & $C_{f}$ & $C_{f}$ \\
$\mathrm{G}$ & $3 C_{f}$ & $4 C_{f}$ & $4 C_{f}$ \\
$\mathrm{H}$ & $3 C_{f}$ & $4 C_{f}$ & $3 C_{f}$ \\
I & $C_{f}$ & $C_{f}$ & $C_{f}$ \\
$\mathrm{~J}$ & \multicolumn{4}{|c|}{$C_{f}$} & $C_{f}$ & $C_{f}$ \\
\hline$C_{f}:$ Cost of floating point operation \\
$C_{s}:$ Cost of square root evaluation \\
\hline
\end{tabular}

\begin{tabular}{|c|c|c|c|c|c|c|c|c|}
\hline \multicolumn{9}{|c|}{ Table 3.C: Cost of Operations $\mathrm{K}$ to $\mathrm{U}$} \\
\hline & \multicolumn{2}{|c|}{$k$ is zero } & \multicolumn{2}{|c|}{$k$ is real } & \multicolumn{2}{|c|}{$k$ is imaginary } & \multicolumn{2}{|c|}{$k$ is complex } \\
\hline Op. & $2 \mathrm{D}$ & $3 \mathrm{D}$ & $2 \mathrm{D}$ & $3 \mathrm{D}$ & $2 \mathrm{D}$ & $3 \mathrm{D}$ & $2 \mathrm{D}$ & $3 \mathrm{D}$ \\
\hline $\mathrm{K}$ & - & - & $C_{f}$ & $C_{f}$ & $C_{f}$ & $C_{f}$ & $2 C_{f}$ & $2 C_{f}$ \\
\hline $\mathrm{L}$ & - & - & $C_{f}$ & $C_{f}$ & $C_{f}$ & $C_{f}$ & $2 C_{f}$ & $2 C_{f}$ \\
\hline M & - & - & $C_{h}$ & $C_{e}$ & $C_{h}$ & $C_{e}$ & $C_{h}$ & $C_{e}$ \\
\hline $\mathrm{N}$ & $C_{l}+C_{f}$ & $C_{f}$ & $2 C_{f}$ & $2 C_{f}$ & $C_{f}$ & $C_{f}$ & $2 C_{f}$ & $2 C_{f}$ \\
\hline 0 & $C_{f}$ & $C_{f}$ & $2 C_{f}$ & $2 C_{f}$ & $C_{f}$ & $C_{f}$ & $2 C_{f}$ & $2 C_{f}$ \\
\hline$P$ & $C_{f}$ & $C_{f}$ & $2 C_{f}$ & $4 C_{f}$ & $C_{f}$ & $C_{f}$ & $4 C_{f}$ & $6 C_{f}$ \\
\hline$Q$ & $C_{f}$ & $C_{f}$ & $2 C_{f}$ & $2 C_{f}$ & $C_{f}$ & $C_{f}$ & $2 C_{f}$ & $2 C_{f}$ \\
\hline $\mathrm{R}$ & $C_{f}$ & $C_{f}$ & $C_{f}$ & $C_{f}$ & $C_{f}$ & $C_{f}$ & $2 C_{f}$ & $2 C_{f}$ \\
\hline$S$ & $C_{f}$ & $C_{f}$ & $2 C_{f}$ & $2 C_{f}$ & $C_{f}$ & $C_{f}$ & $2 C_{f}$ & $2 C_{f}$ \\
\hline $\mathrm{T}$ & $C_{f}$ & $C_{f}$ & $4 C_{f}$ & $4 C_{f}$ & $2 C_{f}$ & $2 C_{f}$ & $8 C_{f}$ & $6 C_{f}$ \\
\hline U & $3 C_{f}$ & $3 C_{f}$ & $6 C_{f}$ & $6 C_{f}$ & $3 C_{f}$ & $3 C_{f}$ & $6 C_{f}$ & $6 C_{f}$ \\
\hline \multicolumn{9}{|c|}{$C_{f}$ : Cost of floating point operation } \\
\hline \multicolumn{9}{|c|}{$C_{s}:$ Cost of square root evaluation } \\
\hline \multicolumn{9}{|c|}{$C_{l}$ : Cost of logarithm evaluation } \\
\hline \multicolumn{9}{|c|}{$C_{h}$ : Cost of Hankel function evaluation } \\
\hline \multicolumn{9}{|c|}{$C_{e}$ : Cost of complex exponential evaluation } \\
\hline
\end{tabular}

The list of operations necessary for computing all the integral operators when $\mathbf{p}$ does not lie on the panel is given in Section 3.4. The computations required for the calculation of each of the integral operators are given Table 3.A. 
Operations $\mathrm{A}$ to $\mathrm{J}$ are independent of $k$ whereas operations $\mathrm{K}$ to $\mathrm{U}$ do depend on the wavenumber. Savings are made in the subroutine when $k$ is zero, purely real or purely imaginary over when $k$ is complex. The computational cost of the set of operations is listed in Tables 3.B and 3.C for each of these cases.

\subsection{Conclusion}

The codes H2LC, H3LC and H3ALC are central to development of the full boundary element method routines in the following Chapters. The subroutines of this Chapter carry out integrations over a given element. The subroutines' parameter lists give the user a great deal of flexibility. The particular Helmholtz operators that are required can be selected and the quadrature rule can be defined within the parameter list.

Within the subroutines a large number of checks on the input data are carried out if the the validation parameter is set LVALID=.TRUE. (see Subsection 3.5.4) and the results from the validation are sent to an external file. In the *BEM* subroutines that follow this validation parameter is switched off since the necessary validation is carried out in the main BEM subroutine.

A boundary element method needs to invoke the relevant $\mathrm{H}^{*} \mathrm{LC}$ core routine hundreds or perhaps thousands of times. In a practical problem with around a thousand elements the core routine will be invoked the order of a million times. For this reason the subroutines are efficiently coded. A computational cost analysis is given to advise the user on the control of processing time.

Using higher order elements would clearly be a useful approach to improving the efficiency of the BEM further. In order to obtain the full benefit from this, a more accurate method of representing the boundary would also need to be included. However, discretising the integral operators in such cases is very difficult, particularly for the $N_{k}$ operator.

We shall see it is very important that we include the $N_{k}$ operator, particularly in the solution of exterior acoustic problems. The techniques for representing the boundary and boundary functions considered in this work are sufficient to approximate all the relevant integral operators and provide a sound basis for the boundary element methods of the next three Chapters. 


\section{Chapter 4}

\section{The Interior Acoustic Problem}

The underlying problem addressed in this Chapter is that of computing the acoustic field within an enclosed homogeneous isotropic fluid subject to a specified boundary condition. Let the fluid occupy an arbitrary closed region $D$ with boundary $S$, as illustrated in Figure 4.1.

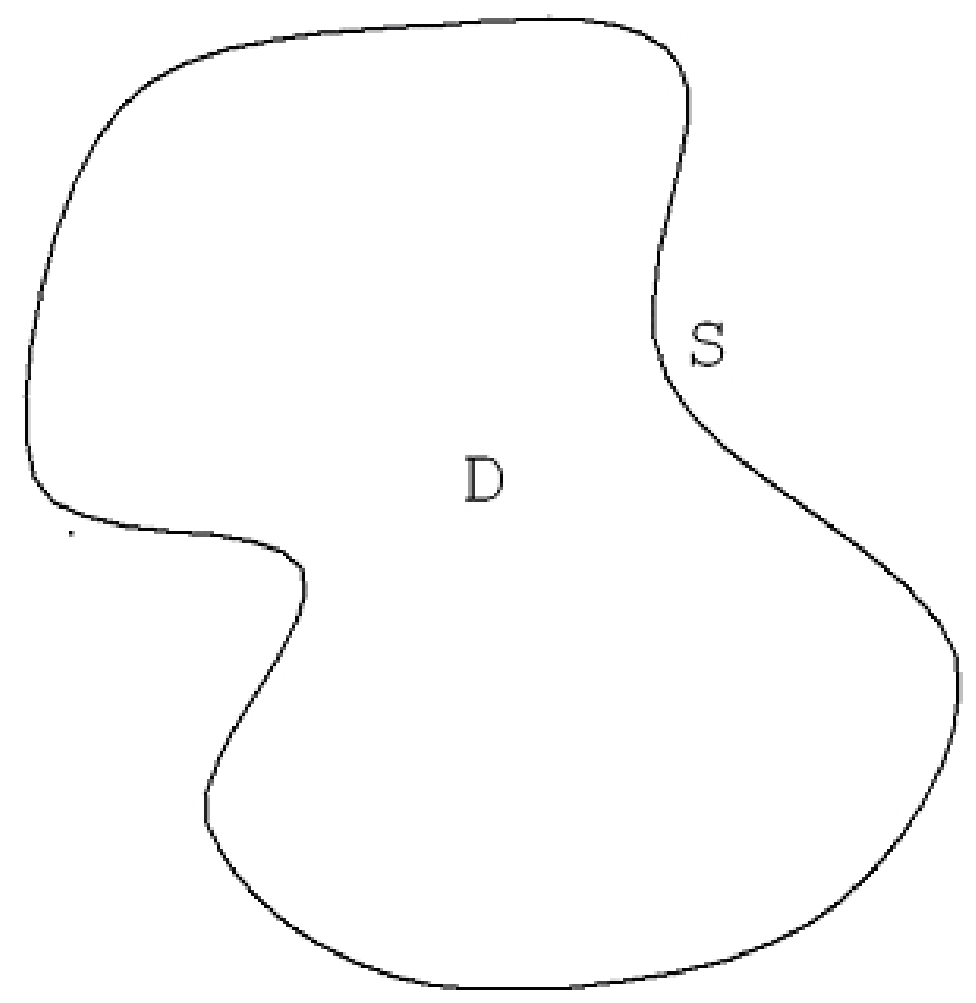

Fig 4.1. The domain of the interior acoustic problem. 
Following the analysis of Subsection 1.3.1, the problem is equivalent to the solution of the Helmholtz equation

$$
\nabla^{2} \varphi(\mathbf{p})+k^{2} \varphi(\mathbf{p})=0 \quad(\mathbf{p} \in D) .
$$

The boundary condition is assumed to take a general form

$$
\alpha(\mathbf{p}) \varphi(\mathbf{p})+\beta(\mathbf{p}) v(\mathbf{p})=f(\mathbf{p})
$$

where $\alpha, \beta$ and $f$ are complex-valued functions defined on the boundary.

In this application the finite element method is now an established computational technique (ref. [68], [69]) and it may well also be often more efficient than the BEM. However, the BEM allows much more flexibility when the geometry of the domain is complicated and is a more natural method to apply if the domain is to be coupled with neighbouring domains in a wider computational method.

The volume of published research into the problem of determining the acoustic field within an enclosure or cavity by the boundary element method is minute in comparison to that of the corresponding exterior problem. There are two important reasons for this. The first is that the interior problem can be solved much more straightforwardly by the finite element method than the exterior problem, hence there is no pressing need for an alternative method. The other reason is that the development of the BEM for the exterior problem has been beset by difficulties; extensive research has been required to achieve reliable methods. The BEM for the interior problem is relatively straightforward.

The boundary element method for the solution of the interior acoustic boundaryvalue problem have been developed in Bell et al [9], Bernard et al [11], and Kipp and Bernard [39]. Further analysis or applications of the method are described in Seybert and Cheng [78], Cheng et al [19] and Kopuz and Lalor [53]. In this Chapter the application of the BEM to the interior acoustic problem is developed further so that the solution with a general boundary, boundary condition and incident field can be obtained. The subroutines AIBEM2, AIBEM3 and AIBEMA [33] for solving the two-, three- and axisymmetric three-dimensional problems are described and demonstrated.

\subsection{Integral Equation Formulation}

In this Section we consider the integral equation fomulations of the interior Helmholtz equation. The range of papers described earlier each consider the solution with a 
particular type of boundary condition such as Dirichlet or Neumann. An incident field along with a general boundary condition (4.1) is included and this leads to more generalised boundary integral equations.

\subsubsection{Direct Formulation}

The application of Green's second theorem to the Helmholtz equation gives the following equations:

$$
\begin{gathered}
\left\{M_{k} \varphi\right\}_{S}(\mathbf{p})+\varphi(\mathbf{p})=\left\{L_{k} v\right\}_{S}(\mathbf{p}) \quad(\mathbf{p} \in D), \\
\left\{M_{k} \varphi\right\}_{S}(\mathbf{p})+\frac{1}{2} \varphi(\mathbf{p})=\left\{L_{k} v\right\}_{S}(\mathbf{p}) \quad(\mathbf{p} \in S),
\end{gathered}
$$

where the Helmholtz integral operators, $L_{k}$ and $M_{k}$, are defined in Section 3.1 and $v(p)=\frac{\partial \varphi}{\partial n}$. The equations have the same structure as those given in Section 1.2 for the interior Laplace equation. Note that the normals to the boundary are taken to be in the outward direction.

The above equations can be utilised to solve the interior Helmholtz equation in the manner outlined in Section 1.2; equation (4.3) gives (approximations to) both $\varphi$ and $v$ on the boundary $S$, equation (4.2) yields an approximation to $\varphi(\mathbf{p})$ for any point $\mathbf{p}$ in the domain. There is only one small difficulty with this approach and that is in the case of a Dirichlet boundary condition equation (4.3) is a Fredholm integral equation of the first kind.

In general first kind equations are found to be difficult to solve and the matrices that arise in their equivalent linear systems are ill-conditioned (see [26], for example). Even though first kind equations like (4.2), having singular kernels, do not present the severe numerical problems that those with smooth kernels do, it is found that their solution can lead to a marginal loss of accuracy since the matrices that arise have higher condition numbers and hence magnify any numerical error [4]. Boundary integral equation reformulations of the interior Helmholtz equation provide us with a selection from which the possibility of having to solve a first kind equations can be avoided.

Differentiating each term of equation (4.2) with respect to any vector $\mathbf{v}(\mathbf{p})$ gives

$$
\frac{\partial}{\partial u_{p}}\left\{M_{k} \varphi\right\}_{S}(\mathbf{p})+\frac{\partial \varphi(\mathbf{p})}{\partial u_{p}}=\frac{\partial}{\partial u_{p}}\left\{L_{k} v\right\}_{S}(\mathbf{p}) \quad(\mathbf{p} \in D),
$$

or

$$
\left\{N_{k} \varphi\right\}_{S}\left(\mathbf{p} ; \mathbf{u}_{p}\right)+\frac{\partial \varphi(\mathbf{p})}{\partial u_{p}}=\left\{M_{k}^{t} v\right\}_{S}\left(\mathbf{p} ; \mathbf{u}_{\mathbf{p}}\right) \quad(\mathbf{p} \in D)
$$


using the notation of Section 3.1.

By taking the limit as the point $\mathbf{p}$ approaches the boundary with the vector $\mathbf{u}_{p}$ being the unit outward normal to the boundary at $\mathbf{p}$ (that is $\mathbf{n}_{p}$ ), and taking into account the jump properties of Section 3.1 the following boundary integral equation is obtained:

$$
\left\{N_{k} \varphi\right\}_{S}\left(\mathbf{p} ; \mathbf{n}_{p}\right)=\left\{\left(M_{k}^{t}-\frac{1}{2} I\right) v\right\}_{S}\left(\mathbf{p} ; \mathbf{n}_{p}\right)(\mathbf{p}) \quad(\mathbf{p} \in S)
$$

where $v(\mathbf{p})=\frac{\partial \varphi(\mathbf{p})}{\partial n_{p}}$.

Since the operators $M_{k}^{t}$ and $N_{k}$ are available through the subroutines in Section 3, then it is straightforward to base the boundary element method on equation (4.4). However, for the Neumann problem we need to solve over the hypersingular operator $N_{k}$, which can lead to some loss of accuracy, similar to that experienced with the solution of the first kind equation discussed earlier.

Since neither of equations (4.3) and (4.4) are universally acceptable for solving the interior Helmholtz equation for all boundary conditions of the form (4.1), a hybrid equation is proposed that couples the two original equations into a single equation

$$
\left\{\left(M_{k}+\frac{1}{2} I+\mu N_{k}\right) \varphi\right\}_{S}\left(\mathbf{p} ; \mathbf{n}_{p}\right)=\left\{\left(L_{k}+\mu\left(M_{k}^{t}-\frac{1}{2} I\right)\right) v\right\}\left(\mathbf{p} ; \mathbf{n}_{p}\right) \quad(\mathbf{p} \in S),
$$

where $\mu(\neq 0)$ is the coupling parameter. The equation (4.5) provides a suitable basis of a method for the solution of interior Helmholtz equation for all boundary conditions and it is the equation employed in the subroutines associated with this Chapter.

\subsubsection{Indirect Formulation}

Following on from the ideas in Subsection 1.2.4, the corresponding indirect integral equation formulations to (4.3) and (4.4) can be obtained by writing $\varphi$ as a single or double layer potential;

$$
\varphi(\mathbf{p})=\left\{L_{k} \sigma_{0}\right\}_{S}(\mathbf{p}) \quad \text { or } \quad \varphi(\mathbf{p})=\left\{M_{k} \sigma_{\infty}\right\}_{S}(\mathbf{p}) \quad(\mathbf{p} \in D)
$$

where the $\sigma_{0}$ and $\sigma_{\infty}$ are source density functions defined on $S$. For points on the boundary the equations become boundary integral equations;

$$
\varphi(\mathbf{p})=\left\{L_{k} \sigma_{0}\right\}_{S}(\mathbf{p}) \quad \text { or } \quad \varphi(\mathbf{p})=\left\{\left(M_{k}-\frac{1}{2} I\right) \sigma_{\infty}\right\}_{S}(\mathbf{p}) \quad(\mathbf{p} \in S)
$$

where the jump condition of Section 3.1 has been taken into account in the second equation. 
The integral equations arrived at in this way have the same difficulties as the corresponding direct equation (4.3); the Dirichlet problem is replaced by a first kind equation. Again the problem can be circumvented by using a hybrid formulation; writing $\varphi$ as a weighted sum of single and double layer potentials

$$
\varphi(\mathbf{p})=\left\{\left(L_{k}+\nu M_{k}\right) \sigma_{\nu}\right\}_{S}(\mathbf{p}) \quad(\mathbf{p} \in D)
$$

where $\nu$ is a weighting parameter. This gives rise to the following boundary integral equation:

$$
\varphi(\mathbf{p})=\left\{\left(L_{k}+\nu\left(M_{k}-\frac{1}{2} I\right)\right) \sigma_{\nu}\right\}_{S}(\mathbf{p}) \quad(\mathbf{p} \in S) .
$$

The parameter $\nu$ should be chosen in a similar way to the parameter $\mu$ in the direct formulation.

The equation (4.7) is only suitable for solving the Dirichlet problem since it refers to $\varphi$ and not $v$ on the boundary. Differentiating equation (4.6) with respect to $\mathbf{n}_{p}$ and taking the limit as the point $\mathbf{p}$ approaches a point on the boundary gives the following boundary integral equation:

$$
v(\mathbf{p})=\left\{\left(M_{k}^{t}+\frac{1}{2} I+\nu N_{k}\right) \sigma_{\nu}\right\}_{S}\left(\mathbf{p} ; \mathbf{n}_{p}\right) \quad(\mathbf{p} \in S),
$$

which can be used for the solution of the Neumann problem.

Both equations (4.7) and (4.8) are required in the indirect solution of the Helmholtz equation with a Robin boundary condition. In this case the relevant integral equation is obtained through the substitution of the forms (4.7) and (4.8) into the boundary condition (4.1) to give

$\alpha(\mathbf{p})\left\{\left(L_{k}+\nu\left(M_{k}-\frac{1}{2} I\right)\right) \sigma_{\nu}\right\}_{S}(\mathbf{p})+\beta(\mathbf{p})\left\{\left(M_{k}^{t}+\frac{1}{2} I+\nu N_{k}\right) \sigma_{\nu}\right\}_{S}\left(\mathbf{p} ; \mathbf{n}_{p}\right)=f(\mathbf{p}) \quad(\mathbf{p} \in S)$.

\subsubsection{Field Modification}

The acoustic field need not be a result of the boundary and boundary condition alone; the surface may simply act to modify an existing field. A simple example of this is that of a loudspeaker in a room; the loudspeaker produces an acoustic field that is modified by the walls of the room. In such cases there is an incident field in the domain, termed $\varphi^{\mathrm{i}}$, which is the field that would exist if there were no boundaries. Such problems can also be solved by the boundary element method, it only requires a generalisation of the integral equations and the corresponding alteration of the boundary element methods. 


\section{Direct formulation}

In the simplest case, the equation (4.2) may be generalised as follows:

$$
\varphi(\mathbf{p})=\varphi^{\mathrm{i}}(\mathbf{p})-\left\{M_{k} \varphi\right\}_{S}(\mathbf{p})+\left\{L_{k} v\right\}_{S}(\mathbf{p}) \quad(\mathbf{p} \in D)
$$

the solution $\varphi(\mathbf{p})$ is equated to the incident field $\varphi^{\mathrm{i}}(\mathbf{p})$ and modified by the other terms. The boundary integral equation that arises from the formulation (4.10) is as follows:

$$
\left\{M_{k} \varphi\right\}_{S}(\mathbf{p})+\frac{1}{2} \varphi(\mathbf{p})=\varphi^{\mathrm{i}}(\mathbf{p})+\left\{L_{k} v\right\}_{S}(\mathbf{p}) \quad(\mathbf{p} \in S) .
$$

The corresponding generalisation of (4.4) is

$$
\left\{N_{k} \varphi\right\}_{S}\left(\mathbf{p} ; \mathbf{n}_{p}\right)=v^{\mathrm{i}}(\mathbf{p})+\left\{\left(M_{k}^{t}-\frac{1}{2} I\right) v\right\}_{S}\left(\mathbf{p} ; \mathbf{n}_{p}\right)_{S}(\mathbf{p}) \quad(\mathbf{p} \in S)
$$

where $v^{\mathrm{i}}(\mathbf{p})=\frac{\partial \varphi^{\mathrm{i}}}{\partial n_{p}}(\mathbf{p})$.

The formulation employed in subroutines AIBEM2, AIBEM3 and AIBEMA is a hybrid of these equations

$$
\left\{\left(M_{k}+\frac{1}{2} I+\mu N_{k}\right) \varphi\right\}_{S}\left(\mathbf{p} ; \mathbf{n}_{p}\right)=\varphi^{\mathrm{i}}(\mathbf{p})+\mu v^{\mathrm{i}}(\mathbf{p})+\left\{\left(L_{k}+\mu\left(M_{k}^{t}-\frac{1}{2} I\right)\right) v\right\}_{S}\left(\mathbf{p} ; \mathbf{n}_{p}\right) \quad(\mathbf{p} \in S) .
$$

The equation (4.11) is the generalisation of (4.5) and the equations are equivalent when there is no incident field $\left(\varphi^{\mathrm{i}}(\mathbf{p})=0, v^{\mathrm{i}}(\mathbf{p})=0\right.$ for all $\left.\mathbf{p} \in D \cup S\right)$.

\section{Indirect formulation}

Generalising equations (4.6)-(4.8) to include the incident field gives rise to the following integral equations:

$$
\begin{gathered}
\varphi(\mathbf{p})=\varphi^{\mathbf{i}}(\mathbf{p})+\left\{\left(L_{k}+\nu M_{k}\right) \sigma_{\nu}\right\}_{S}(\mathbf{p}) \quad(\mathbf{p} \in D) \\
\varphi(\mathbf{p})=\varphi^{\mathbf{i}}(\mathbf{p})+\left\{\left(L_{k}+\nu\left(M_{k}-\frac{1}{2} I\right)\right) \sigma_{\nu}\right\}_{S}(\mathbf{p}) \quad(\mathbf{p} \in S) \\
v(\mathbf{p})=v^{\mathrm{i}}(\mathbf{p})+\left\{\left(M_{k}^{t}+\frac{1}{2} I+\nu N_{k}\right) \sigma_{\nu}\right\}_{S}\left(\mathbf{p} ; \mathbf{n}_{p}\right) \quad(\mathbf{p} \in S) .
\end{gathered}
$$

The indirect boundary integral equation for the solution of the interior Helmholtz equation with the general Robin boundary condition (4.1) and with an incident field is as follows:

$\alpha(\mathbf{p})\left\{\varphi^{\mathrm{i}}(\mathbf{p})+\left(L_{k}+\nu\left(M_{k}-\frac{1}{2} I\right)\right) \sigma_{\nu}\right\}(\mathbf{p})+\beta(\mathbf{p})\left\{v^{\mathrm{i}}(\mathbf{p})+\left(M_{k}^{t}+\frac{1}{2} I+\nu N_{k}\right) \sigma_{\nu}\right\}_{S}\left(\mathbf{p} ; \mathbf{n}_{p}\right)=f(\mathbf{p})$

for $(\mathbf{p} \in S)$. 


\subsection{Boundary Element Method}

By approximating the operators in the boundary integral equations in the way described in Section 3.3, the equations can each be reduced to a linear system of equations, as demonstrated in Section 1.2. The first step is to approximate the boundary $S$ by a set of $n$ panels $\tilde{S}=\sum_{j=1}^{n} \Delta \tilde{S}_{j}$, as described in Chapter 2. The integral operators are approximated by the technique outlined in Chapter 3 and the resulting linear system of equations is solved. The overall method is equivalent to the solution of the integral equations by collocation.

\subsubsection{Direct Boundary Element Method}

The application of collocation to the integral equation (4.11) reduces it to the following linear system of equations:

$$
\left[\mathrm{M}_{k}+\frac{1}{2} \mathrm{I}+\mu \mathrm{N}_{k}\right] \underline{\varphi} \approx \underline{\varphi}^{\mathrm{i}}+\mu \underline{v}^{\mathrm{i}}+\left[\mathrm{L}_{k}+\mu\left(\mathrm{M}_{k}^{t}-\frac{1}{2} \mathrm{I}\right)\right] \underline{v}
$$

The $\mathrm{L}_{k}, \mathrm{M}_{k}, \mathrm{M}_{k}^{t}$ and $\mathrm{N}_{k}$ are $n \times n$ matrices arising from the discretisation method outlined in Section 1.2 and Chapter 3; for example the components of $\mathrm{L}_{k}$ are defined by $\left[\mathrm{L}_{k}\right]_{i j}=\left\{L_{k} \tilde{e}\right\}_{\Delta \tilde{S}_{j}}\left(\mathbf{p}_{i}\right)$, where $\tilde{e}$ is the unit function.

The vectors $\underline{\varphi}$ and $\underline{v}$ represent the values of the boundary functions $\varphi$ and $v$ at the collocation points. The method involves finding the solution of the linear system of equations

$$
\left[\mathrm{M}_{k}+\frac{1}{2} \mathrm{I}+\mu \mathrm{N}_{k}\right] \underline{\hat{\varphi}}=\underline{\varphi}^{\mathrm{i}}+\mu \underline{v}^{\mathrm{i}}+\left[\mathrm{L}_{k}+\mu\left(\mathrm{M}_{k}^{t}-\frac{1}{2} \mathrm{I}\right)\right] \underline{\hat{v}}
$$

subject to the boundary condition applied at the collocation points;

$$
\alpha_{i} \hat{\varphi}_{i}+\beta_{i} \underline{\hat{v}}_{i}=f_{i} \quad \text { for } \quad i=1,2, \ldots, n \quad \text { or } \quad \mathrm{D}_{\alpha} \underline{\hat{\varphi}}+\mathrm{D}_{\beta} \underline{\hat{v}}=\underline{f},
$$

with $\alpha_{i}=\alpha\left(\mathbf{p}_{i}\right), \beta_{i}=\beta\left(\mathbf{p}_{i}\right), f_{i}=f\left(\mathbf{p}_{i}\right)$ and the $\mathrm{D}_{\alpha}$ and $\mathrm{D}_{\beta}$ denote diagonal matrices with $\left[\mathrm{D}_{\alpha}\right]_{i i}=\alpha_{i}$ and $\left[\mathrm{D}_{\beta}\right]_{i i}=\beta_{i}$, to find $\underline{\hat{\varphi}}$ and $\underline{\hat{\hat{v}}}$.

In the cases of a pure Dirichlet or pure Neumann boundary condition the equations can be solved by a standard method such as LU factorization or Gaussian elimination. However in the general case the equations (4.17) can be applied to rearrange the linear system of equations (5.27) and the matrix-vector equation that arises can then be solved by standard methods. The method used for solving systems of equations of the form (4.17), (4.18) is described in Appendix 3. 
Once $\underline{\hat{\varphi}}$ and $\underline{\hat{v}}$ are obtained, equation (4.10) can be used to return an approximation to the solution at any point $\mathbf{p}$ in the domain;

$$
\hat{\varphi}(\mathbf{p})=\varphi^{\mathrm{i}}(\mathbf{p})-\sum_{j=1}^{n}\left\{M_{k} \tilde{e}\right\}_{\Delta S_{j}}(\mathbf{p}) \hat{v}_{j}+\sum_{j=1}^{n}\left\{L_{k} \tilde{e}\right\}_{\Delta S_{j}}(\mathbf{p}) \hat{\varphi}_{j}
$$

\subsubsection{Indirect Boundary Element Method}

The collocation method reduces the indirect boundary integral equations $(4.13),(4.14)$ to the linear systems of approximations

$$
\begin{gathered}
\underline{\varphi} \approx \underline{\varphi}^{\mathrm{i}}+\left[\mathrm{L}_{k}+\nu\left(\mathrm{M}_{k}-\frac{1}{2} \mathrm{I}\right)\right] \underline{\sigma}_{\nu} \quad \text { and } \\
\underline{v} \approx \underline{v}^{\mathrm{i}}+\left[\mathrm{M}_{k}^{t}+\frac{1}{2} \mathrm{I}+\nu \mathrm{N}_{k}\right] \underline{\sigma}_{\nu} .
\end{gathered}
$$

Applying the boundary condition at the collocation points as in the direct method gives the equation

$$
\mathrm{D}_{\alpha} \underline{\varphi}+\mathrm{D}_{\beta} \underline{v}=\underline{f} .
$$

Substituting the approximations for $\underline{\varphi}$ and $\underline{v}$ given above into equation (4.18) gives the following:

$$
\left[\mathrm{D}_{\alpha}\left\{\mathrm{L}_{k}+\nu\left(\mathrm{M}_{k}-\frac{1}{2} \mathrm{I}\right)\right\}+\mathrm{D}_{\beta}\left\{\mathrm{M}_{k}^{t}+\frac{1}{2} \mathrm{I}+\nu \mathrm{N}_{k}\right\}\right] \underline{\sigma}_{\nu}+\mathrm{D}_{\alpha} \underline{\varphi}^{\mathrm{i}}+\mathrm{D}_{\beta} \underline{v}^{\mathrm{i}} \approx \underline{f}
$$

which is also the discrete analogue of equation (4.15).

In the indirect boundary element method, the first stage is to find the approximation $\hat{\sigma}_{\nu}$ to the source density function $\sigma$. This can be done through the solution of the following linear system of equations:

$$
\left[\mathrm{D}_{\alpha}\left\{\mathrm{L}_{k}+\nu\left(\mathrm{M}_{k}-\frac{1}{2} \mathrm{I}\right)\right\}+\mathrm{D}_{\beta}\left\{\mathrm{M}_{k}^{t}+\frac{1}{2} \mathrm{I}+\nu \mathrm{N}_{k}\right\}\right] \underline{\hat{\sigma}}_{\nu}+\mathrm{D}_{\alpha} \underline{\varphi}^{\mathrm{i}}+\mathrm{D}_{\beta} \underline{v}^{\mathrm{i}}=\underline{f} .
$$

The form of this equation is more straightforward than the corresponding equations for the direct method (4.17), (4.18). The equation (4.21) is simply a matrix-vector equation that can be immediately solved by Gaussian elimination-type methods. Having obtained the solution to (4.21), the approximation to $\underline{\sigma}_{\nu}$, the approximate solution in the domain can be found using the discrete equivalent of (4.12);

$$
\hat{\varphi}(\mathbf{p})=\varphi^{\mathrm{i}}(\mathbf{p})+\sum_{j=1}^{n}\left(\left\{L_{k} \tilde{e}\right\}_{\Delta S_{j}}+\nu\left\{M_{k} \tilde{e}\right\}_{\Delta S_{j}}\right) \hat{\sigma}_{\nu j} .
$$


www.boundary-element-method.com

\subsection{Subroutines AIBEM2, AIBEM3 and AIBEMA}

In this Section the subroutines AIBEM2, AIBEM3 and AIBEMA are introduced. The purpose of the subroutines is to solve the interior acoustic problem. Each subroutine's parameter list has the following general form:

SUBROUTINE AIBEM $\{2$ or 3 or $\mathrm{A}(\}$

real wavenumber, description of boundary and set of interior solution points, boundary condition, incident field at boundary points and at interior solution points, control and validation parameters, solution at boundary points and at interior solution points (solution), working space )

The subroutines require input of the conditions of the acoustic field - the wavenumber, a geometrical description of the boundary of the domain (as covered in Chapter 2) and a list of the points in the interior domain where a solution is sought, the boundary condition and the incident field (if any). The subroutine returns the solution at the boundary points and at the selected points in the domain. The uses of the subroutines are demonstrated by the programs AIBEM2_T, AIBEM3_T and AIBEMA_T in the next Section.

\subsubsection{Solution Strategy of the AIBEM* routines}

In the AIBEM* routines the interior Helmholtz equation is solved by the improved direct boundary element method. That is finding the solution of (4.17) subject to the discrete boundary condition (4.18). This will result in obtaining (approximations to) both $\varphi$ and $v$ on $S$. The solution in the domain is then found using equation (4.19).

A simple analysis of the magnitude of the integral operators suggests that a weighting of the form $\mu \sim \frac{1}{k}$ tends to ensure that the relative contribution from each integral operator on either side of the equation remains in balance, whatever the value of $k$. $\mu$ is a parameter in the Fortran subroutines for solving the interior acoustic problem. In the test problems the parameter is chosen as follows:

$$
\mu=\frac{i}{k+1} .
$$




\subsubsection{Subroutine AIBEM2}

Subroutine AIBEM2 computes the solution of the two-dimensional Helmholtz equation in the domain interior to a closed boundary. The boundary $(S)$ is approximated by a set of straight line elements.

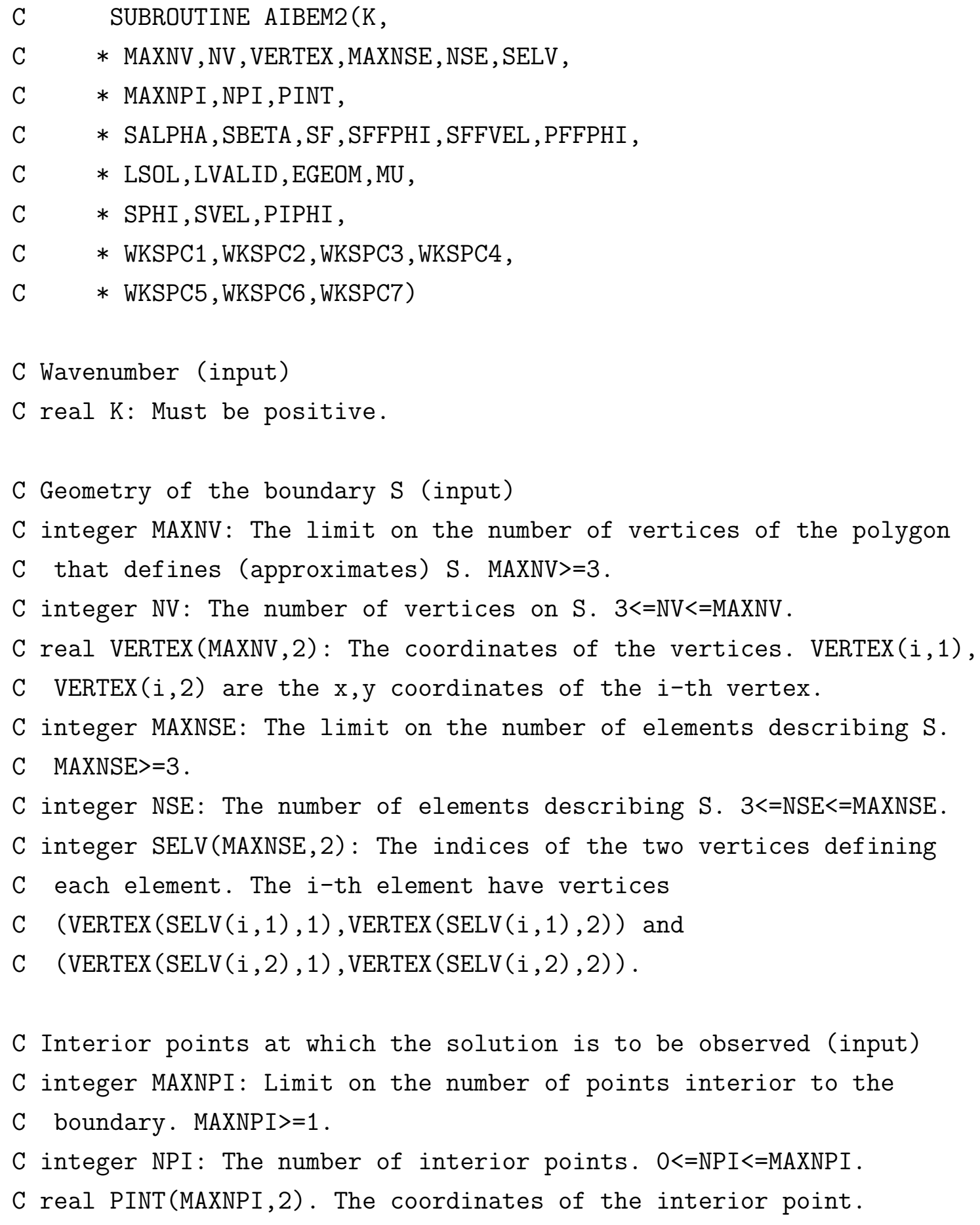


C PINT( $(i, 1), \operatorname{PINT}(i, 2)$ are the $\mathrm{x}, \mathrm{y}$ coordinates of the $i$-th point.

C The boundary condition ( $\{\backslash$ alpha $\}\{\backslash$ phi $\}+\{\backslash$ beta $\} V=f)$ (input)

C complex SALPHA(MAXNSE): The values of $\{\backslash$ alpha\} at the centres

$\mathrm{C}$ of the elements.

C complex SBETA(MAXNSE): The values of $\{\backslash$ beta $\}$ at the centres

$\mathrm{C}$ of the elements.

C complex SF(MAXNSE): The values of $f$ at the centres of the

C elements.

C complex SFFPHI (MAXNSE): The incident velocity potential at the

$\mathrm{C}$ centres of the elements

C complex SFFVEL(MAXNSE): The derivative of the incident velocity

C centres of the elements

C complex PFFPHI(MAXNPI): The incident velocity potential at the chosen

$\mathrm{C}$ interior points

C Validation and control parameters (input)

C logical LSOL: A switch to control whether the particular solution is

C required

C logical LVALID: A switch to enable the choice of checking of

C subroutine parameters.

C real EGEOM: The maximum absolute error in the parameters that

C describe the geometry.

C complex MU: The weighting parameter in the direct formulations.

C As a default, set $M U=I /(K+1)$.

C Solution (output)

C complex SPHI(MAXNSE): The velocity potential ("\{\phi\}") at the

C centres of the boundary elements.

C complex SVEL(MAXNSE): The velocity ("v" or "d $\{\backslash p h i\} / d n "$ where $\mathrm{n}$ is

$\mathrm{C}$ the outward normal to the boundary) at the centres of the boundary

C elements.

C complex PIPHI(MAXNPI): The velocity potential ("\{\phi\}") at the

$\mathrm{C}$ interior points.

C Working space 


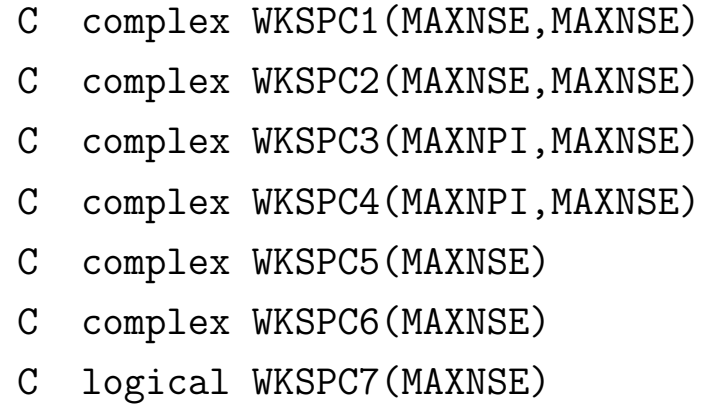

The subroutine parameters that specify the interior two-dimensional Helmholtz problem must be set up in the main program. Let this be called MAIN.FOR. The following files must be linked together to construct the complete program:

$M A I N . F O R$ (and files containing any user-defined sub-programs),

AIBEM2.FOR,

H2LC.FOR, the file for computing the discrete operators - see Chapter 3,

FNHANK.FOR, the Hankel function - see Appendix 5,

CGLS.FOR, the file for computing the solution to a linear system - see Appendix 3, GEOM2D.FOR, the file for 2D geometry - see Appendix 6.

\subsubsection{Subroutine AIBEM3}

Subroutine AIBEM3 computes solution of the three-dimensional Helmholtz equation in the domain interior to a closed surface. The boundary $(S)$ is approximated by a set of planar triangular elements.

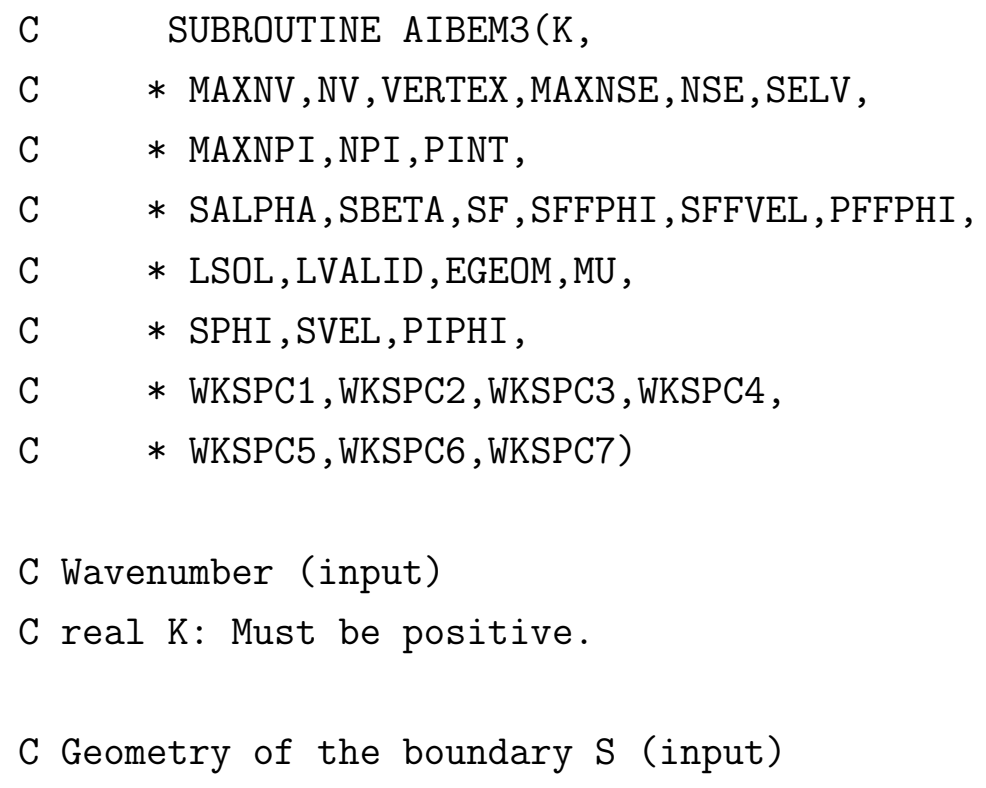


$C$ integer MAXNV: The limit on the number of vertices of the polygon

C that defines (approximates) S. MAXNV $>=4$.

$\mathrm{C}$ integer NV: The number of vertices on $\mathrm{S} .4<=\mathrm{NV}<=\mathrm{MAXNV}$.

C real VERTEX(MAXNV,3): The coordinates of the vertices. VERTEX $(i, 1)$,

C VERTEX $(i, 2), \operatorname{VERTEX}(i, 3)$ are the $x, y, z$ coordinates of the $i$-th

C vertex.

$C$ integer MAXNSE: The limit on the number of elements describing $\mathrm{S}$.

C MAXNSE $>=4$.

$C$ integer NSE: The number of elements describing $\mathrm{S} .4<=\mathrm{NSE}<=\mathrm{MAXNSE}$.

$C$ integer SELV(MAXNSE,3): The indices of the three vertices defining

$\mathrm{C}$ each element. The i-th element have vertices

C $(\operatorname{Vertex}(\operatorname{SelV}(i, 1), 1), \operatorname{Vertex}(\operatorname{SelV}(i, 1), 2)), \operatorname{Vertex}(\operatorname{SelV}(i, 1), 3))$,

C $(\operatorname{Vertex}(\operatorname{SeLV}(i, 2), 1), \operatorname{VerteX}(\operatorname{SelV}(i, 2), 2)), \operatorname{VerteX}(\operatorname{SelV}(i, 2), 3))$ and

C $(\operatorname{VerteX}(\operatorname{SELV}(i, 3), 1), \operatorname{VerteX}(\operatorname{SELV}(i, 3), 2)), \operatorname{VerteX}(\operatorname{SELV}(i, 3), 3))$.

C Interior points at which the solution is to be observed (input)

$C$ integer MAXNPI: Limit on the number of points interior to the

C boundary. MAXNPI $>=1$.

$C$ integer NPI: The number of interior points. $0<=N P I<=M A X N P I$.

$C$ real PINT(MAXNPI,3). The coordinates of the interior point.

C PINT(i,1),PINT $(i, 2), \operatorname{PINT}(i, 3)$ are the $x, y, z$ coordinates of the $i$-th

C point.

C The boundary condition ( $\{\backslash$ alpha $\}$ phi $+\{\backslash$ beta $\} \mathrm{v}=\mathrm{f}$ ) (input)

C complex SALPHA(MAXNSE): The values of $\{\backslash$ alpha\} at the centres

C of the elements.

C complex SBETA(MAXNSE): The values of "\{\beta\}" at the centres

$C$ of the elements.

C complex SF(MAXNSE): The values of "f" at the centres of the

C elements.

C complex SFFPHI(MAXNSE): The incident velocity potential at the

$C$ centres of the elements

C complex SFFVEL(MAXNSE): The derivative of the incident velocity

C centres of the elements

C complex PFFPHI(MAXNPI): The incident velocity potential at the chosen

$\mathrm{C}$ interior points 
C Validation and control parameters (input)

C logical LSOL: A switch to control whether the particular solution is

C required

C logical LVALID: A switch to enable the choice of checking of

C subroutine parameters.

C real EGEOM: The maximum absolute error in the parameters that

C describe the geometry.

C complex MU: The weighting parameter in the direct formulations.

C As a default, set $M U=I /(K+1)$.

C Solution (output)

C complex SPHI(MAXNSE): The velocity potential ( $\backslash$ phi\}) at the

$C$ centres of the boundary elements.

C complex SVEL(MAXNSE): The velocity ( $v$ or $d\{\backslash p h i\} / d n$ where $n$ is

$C$ the outward normal to the boundary) at the centres of the boundary

C elements.

C complex PIPHI(MAXNPI): The velocity potential (\{\phi\}) at the

$\mathrm{C}$ interior points.

C Working space

C complex WKSPC1 (MAXNSE, MAXNSE)

C complex WKSPC2(MAXNSE, MAXNSE)

C complex WKSPC3(MAXNPI, MAXNSE)

C complex WKSPC4(MAXNPI, MAXNSE)

C complex WKSPC5(MAXNSE)

C complex WKSPC6 (MAXNSE)

C logical WKSPC7(MAXNSE)

The subroutine parameters that specify the interior three-dimensional Helmholtz problem must be set up in the main program. Let this be called MAIN.FOR. The following files must be linked together to construct the complete program:

$M A I N . F O R$ (and files containing any user-defined sub-programs),

AIBEM3.FOR,

H3LC.FOR, the file for computing the discrete operators - see Chapter 3,

CGLS.FOR, the file for computing the solution to a linear system - see Appendix 3, GEOM3D.FOR, the file for 3D geometry - see Appendix 6. 


\subsubsection{Subroutine AIBEMA}

Subroutine AIBEMA computes solution of the three-dimensional axisymmetric Helmholtz equation in the domain interior to a closed surface. The boundary $(S)$ is approximated by a set of truncated cone elements.

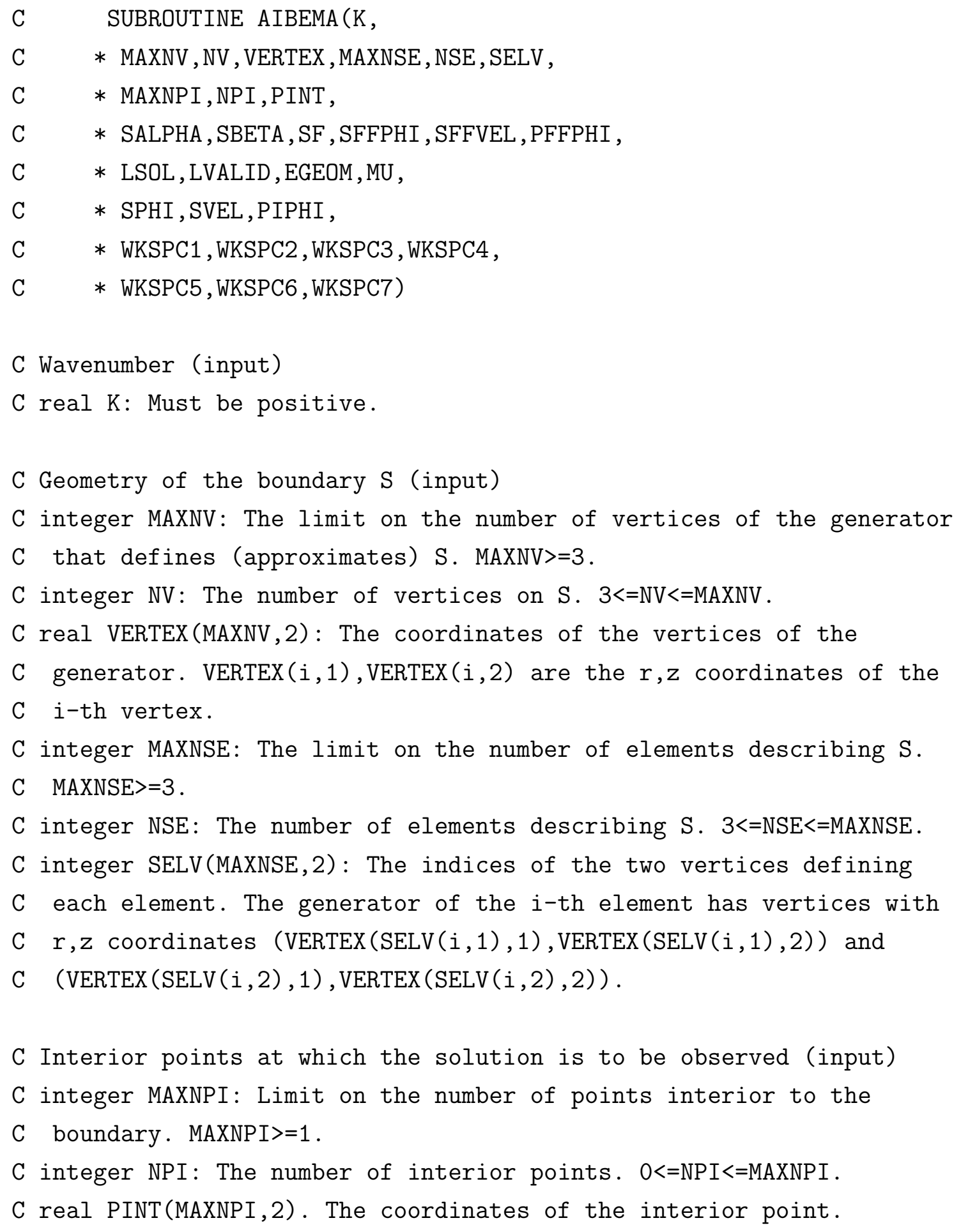


C PINT( $i, 1), \operatorname{PINT}(i, 2)$ are the $r, z$ coordinates of the $i$-th point.

C The boundary condition ( $\{\backslash$ alpha $\}\{\backslash p h i\}+\{\backslash$ beta $\} v=f$ ) (input)

C complex SALPHA(MAXNSE): The values of $\{\backslash$ alpha\} at the centres

$C$ of the generator of the elements.

C complex SBETA(MAXNSE): The values of $\{\backslash$ beta $\}$ at the centres

$\mathrm{C}$ of the generator of the elements.

C complex SF(MAXNSE): The values of $f$ at the centres of the

$C$ of the generator of the elements.

C complex SFFPHI(MAXNSE): The incident velocity potential at the

C centres of the generator of the elements

C complex SFFVEL(MAXNSE): The derivative of the incident velocity

C centres of the generator of the elements

C complex PFFPHI(MAXNPI): The incident velocity potential at the chosen

$\mathrm{C}$ interior points

C Validation and control parameters (input)

C logical LSOL: A switch to control whether a particular solution

$\mathrm{C}$ is required.

C logical LVALID: A switch to enable the choice of checking of

C subroutine parameters.

C real EGEOM: The maximum absolute error in the parameters that

C describe the geometry.

C complex MU: The weighting parameter in the direct formulations.

C As a default, set $\mathrm{MU}=\mathrm{I} /(\mathrm{K}+1)$.

C Solution (output)

C complex SPHI (MAXNSE): The velocity potential ( $\backslash$ phi $\}$ ) at the

C centres of the boundary elements.

C complex SVEL(MAXNSE): The velocity ( $\mathrm{v}$ or $\mathrm{d}\{\backslash \mathrm{phi}\} / \mathrm{dn}$ where $\mathrm{n}$ is

$C$ the outward normal to the boundary) at the centres of the boundary

C elements.

C complex PIPHI(MAXNPI): The velocity potential (\{\phi\}) at the

C interior points.

C Working space 


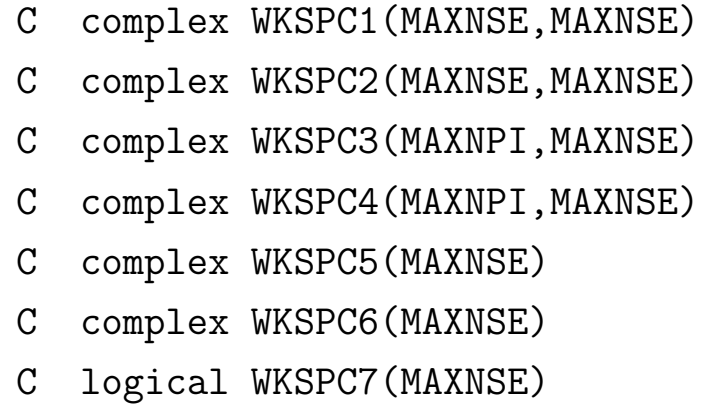

The subroutine parameters that specify the interior three-dimensional axisymmetric Helmholtz problem must be set up in the main program. Let this be called MAIN.FOR. The following files must be linked together to construct the complete program:

MAIN.FOR (and files containing any user-defined sub-programs), AIBEMA.FOR, H3ALC.FOR, the file for computing the discrete operators - see Chapter 3, CGLS.FOR, the file for computing the solution to a linear system - see Appendix 3, GEOM2D.FOR, the file for 2D geometry - see Appendix 6, GEOM3D.FOR, the file for 3D geometry - see Appendix 6.

\subsection{Test Programs}

In this Section the subroutines AIBEM2, AIBEM3 and AIBEMA are demonstrated through invoking them from a main program and comparing the results with analytic solutions. The corresponding main programs are AIBEM2_T, AIBEM3_T and AIBEMA_T.

\subsubsection{Program AIBEM2_T}

The main program AIBEM2_T tests module AIBEM2, the subroutine for computing the solution of the Helmholtz equation interior to a closed boundary in a two-dimensional domain. In AIBEM2_T the domain is the interior of a square of side $0.1 \mathrm{~m}$ with the boundary represented by 32 uniform elements. The representation of the boundary is described fully in Section 2.2 by the data structures VERTEX and SELV, see Tables 2.A and 2.B. Full results are given in file AIBEM2.OUT.

The acoustic medium is air at 20 celcius and 1 atmosphere. The speed of sound is assigned the value $c=344 \mathrm{~m} / \mathrm{s}$. The chosen frequency of the test is $400 \mathrm{~Hz}$, hence 
$k=7.31$. The velocity potential defined by

$$
\varphi(\mathbf{p})=\sin \left(\frac{k}{\sqrt{2}} p_{1}\right) \sin \left(\frac{k}{\sqrt{2}} p_{2}\right)
$$

is clearly a solution of the Helmholtz equation. The boundary velocity is given by differentiating $\varphi$ with respect to $p_{1}$ on the lines $p_{1}=0$ and $p_{1}=0.1$ and with respect to $p_{2}$ on the lines $p_{2}=0, p_{2}=0.1$ and taking into account the direction of the outward normal on these boundary lines. This gives the following surface velocities on each side of the square:

$$
v(\mathbf{p})=\left\{\begin{array}{cl}
0 & \text { when } p_{1}=0, \\
0 & \text { when } p_{2}=0 \\
\frac{k}{\sqrt{2}} \cos \left(\frac{k}{\sqrt{2}} 0.1\right) \sin \left(\frac{k}{\sqrt{2}} p_{2}\right) & \text { when } p_{1}=0.1 \\
\frac{k}{\sqrt{2}} \sin \left(\frac{k}{\sqrt{2}} p_{1}\right) \cos \left(\frac{k}{\sqrt{2}} 0.1\right) & \text { when } p_{2}=0.1 .
\end{array}\right.
$$

Two test problems are devised in which the boundary condition

$$
\alpha(\mathbf{p}) \varphi(\mathbf{p})+\beta(\mathbf{p}) v(\mathbf{p})=f(\mathbf{p})
$$

are set up so that $\alpha(\mathbf{p})=1, \beta(\mathbf{p})=0, f(\mathbf{p})=\varphi(\mathbf{p})$ for $\mathbf{p} \in S$, the Dirichlet condition and $\alpha(\mathbf{p})=0, \beta(\mathbf{p})=1, f(\mathbf{p})=v(\mathbf{p})$ for $\mathbf{p} \in S$, the Neumann condition. The numerical solution is determined on the boundary of the domain and at the interior points $(0.025,0.025),(0.075,0.025),(0.075,0.025),(0.075,0.075),(0.05,0.05)$.

To test run the program, link the files AIBEM2_T.FOR, AIBEM2.FOR, H2LC.FOR, GEOM2D.FOR, CGLS.FOR and the file FNHANK (or the equivalent). The output file is AIBEM2.OUT. The exact and numerical solutions at the selected interior points are given in the Table 4.A.

\begin{tabular}{|c||c|c|c|}
\hline \multicolumn{4}{|c|}{ Table 4.A: Results from AlBEM2_T } \\
\hline point & exact solution & $\begin{array}{c}\text { numerical solution } \\
\text { to Dirichlet condition }\end{array}$ & $\begin{array}{c}\text { numerical solution } \\
\text { to Neumann condition }\end{array}$ \\
\hline$(0.025,0.025)$ & 0.0166 & $0.0159+i 0.0001$ & $0.0156-i 0.0012$ \\
$(0.075,0.025)$ & 0.0488 & $0.0482-i 0.0000$ & $0.0480-i 0.0014$ \\
$(0.025,0.075)$ & 0.0488 & $0.0482-i 0.0000$ & $0.0480-i 0.0014$ \\
$(0.075,0.075)$ & 0.1429 & $0.1434-i 0.0003$ & $0.1398-i 0.0019$ \\
$(0.050,0.050)$ & 0.0653 & $0.0650-i 0.0000$ & $0.0639-i 0.0015$ \\
\hline
\end{tabular}




\subsubsection{Program AIBEM3_T}

The main program AIBEM3_T tests module AIBEM3, the subroutine for computing the solution of the Helmholtz equation interior to a general closed surface in a threedimensional domain. In AIBEM3_T the domain is the interior of a sphere of side $1 \mathrm{~m}$ with the boundary represented by 36 elements. The representation of the boundary is described fully in Section 2.3 by the data structures VERTEX and SELV, see Tables 2.C and 2.D. Full results are given in file AIBEM3.OUT.

The acoustic medium is air at 20 celcius and 1 atmosphere so that the speed of sound is $c=344 \mathrm{~m} / \mathrm{s}$. The chosen frequency of the test is $20 \mathrm{~Hz}$, hence $k=0.37$. The velocity potential defined by

$$
\varphi(\mathbf{p})=\sin \left(k p_{3}\right)
$$

is clearly a solution of the Helmholtz equation. The surface velocity at a point $\mathbf{p}$ is given by differentiating $\varphi$ with respect the outward normal there, $\mathbf{n}_{p}$, to give

$$
\frac{\partial \varphi}{\partial n_{p}}=\underline{\nabla} \cdot \mathbf{n}_{p}=\frac{\partial \varphi}{\partial p_{3}} n_{3}=k \cos \left(k p_{3}\right) n_{3} .
$$

where $\mathbf{n}_{p}=\left(n_{1}, n_{2}, n_{3}\right)$. Two test problems are devised in which the boundary condition

$$
\alpha(\mathbf{p}) \varphi(\mathbf{p})+\beta(\mathbf{p}) v(\mathbf{p})=f(\mathbf{p})
$$

are set up so that $\alpha(\mathbf{p})=1, \beta(\mathbf{p})=0, f(\mathbf{p})=\varphi(\mathbf{p})$ for $\mathbf{p} \in S$, the Dirichlet condition and $\alpha(\mathbf{p})=0, \beta(\mathbf{p})=1, f(\mathbf{p})=v(\mathbf{p})$ for $\mathbf{p} \in S$, the Neumann condition. The numerical solution is determined on the boundary of the domain and at the interior points $(0.5,0.0,0.0),(0.0,0.0,0.25),(0.0,0.0,0.5),(0.0,0.0,0.75)$.

The sound pressure in this example is given by multiplying the velocity potential by $i \rho \omega=2 \pi i \rho f$. In this example $\rho=1.205$ and $f=100 \mathrm{~Hz}$ hence the sound pressure is returned when the velocity potentials are multiplied by $i 757.12$;

$$
p(\mathbf{p})=i 757.12 \varphi(\mathbf{p})
$$

The sound pressures corresponding to the velocity potentials at each solution point are given in the output file and some of the results are reproduced in Table 4.B. 


\begin{tabular}{|c||c|c|c|}
\hline \multicolumn{3}{|c|}{ Table 4.B: Results from AIBEM3_T } \\
\hline point & exact solution & $\begin{array}{c}\text { numerical solution } \\
\text { to Dirichlet condition }\end{array}$ & $\begin{array}{c}\text { numerical solution } \\
\text { to Neumann condition }\end{array}$ \\
\hline$(0.5,0.0,0.0)$ & 0.0000 & $0.0000-i 0.0000$ & $0.0000-i 0.0000$ \\
$(0.0,0.0,0.25)$ & 0.0912 & $0.0889-i 0.0008$ & $0.0800+i 0.0013$ \\
$(0.0,0.0,0.5)$ & 0.1816 & $0.1757-i 0.0015$ & $0.1586+i 0.0025$ \\
$(0.0,0.0,0.75)$ & 0.2706 & $0.2623-i 0.0020$ & $0.2377+i 0.0035$ \\
\hline
\end{tabular}

In the final example that of finding the velocity potential at points in the domain of the sphere with the Neumann boundary condition

$$
v(\mathbf{p})=0 \quad \text { for } \quad \mathbf{p} \in S
$$

and with a point source at the centre of the sphere, giving the incident field

$$
\varphi(\mathbf{p})^{\mathrm{i}}=\frac{1}{4 \pi r} e^{i k r}
$$

The velocity potentials obtained at the interior points are summarised in Table 4.C.

\begin{tabular}{|c||c|c|}
\hline \multicolumn{3}{|c|}{ Table 4 C: Results from AIBEM3_T } \\
\hline point & incident field & numerical solution \\
\hline$(0.0,0.0,0.25)$ & $0.317+\mathrm{i} 0.029$ & $-1.143-\mathrm{i} 0.594$ \\
$(0.0,0.0,0.5)$ & $0.157+\mathrm{i} 0.029$ & $-1.296-\mathrm{i} 0.590$ \\
$(0.0,0.0,0.75)$ & $0.102+\mathrm{i} 0.029$ & $-1.372-\mathrm{i} 0.599$ \\
\hline
\end{tabular}

\subsubsection{Program AIBEMA_T}

The main program AIBEMA_T tests module AIBEMA. In AIBEMA_T the acoustic domain is interior of a sphere of unit radius. The surface is represented by eighteen truncated conical elements, as described in 2.4. Program AIBEM3_T runs three test problems. In each test problem the frequency is $40 \mathrm{~Hz}$ and the acoustic medium is air at 20 celcius $(\mathrm{c}=344 \mathrm{~m} / \mathrm{s})$. Full results are given in file AIBEMA.OUT.

For each test the solution is listed on the surface. In the first test problem the velocity potential on the surface is determined by the field

$$
\varphi(\mathbf{p})=\sin \left(k p_{3}\right)
$$


www.boundary-element-method.com

and AIBEMA is used to determine $\varphi$ within the domain. The results are given in Table 4.D.

\begin{tabular}{|c||c|c|}
\hline \multicolumn{3}{|c|}{ Table 4.D: Results from AIBEMA_T } \\
\hline point $(\mathrm{r}, \mathrm{z})$ & exact solution & numerical solution \\
\hline$(0.0,0.0)$ & 0.000 & $0.000-\mathrm{i} 0.001$ \\
$(0.0,0.5)$ & 0.357 & $0.357+\mathrm{i} 0.004$ \\
$(0.0,-0.5)$ & -0.357 & $-0.357-\mathrm{i} 0.004$ \\
$(0.5,0.0)$ & 0.000 & $0.000-\mathrm{i} 0.000$ \\
\hline
\end{tabular}

The second test problem has the same solution but in this case the corresponding Neumann condition is applied at the boundary. The corresponding solutions from this test problem are given in Table 4.E.

\begin{tabular}{|c||c|c|}
\hline \multicolumn{3}{|c|}{ Table 4.E: Results from AIBEMA_T } \\
\hline point $(\mathrm{r}, \mathrm{z})$ & exact solution & numerical solution \\
\hline$(0.0,0.0)$ & 0.000 & $0.000-\mathrm{i} 0.000$ \\
$(0.0,0.5)$ & 0.357 & $0.354-\mathrm{i} 0.001$ \\
$(0.0,-0.5)$ & -0.357 & $-0.354-\mathrm{i} 0.001$ \\
$(0.5,0.0)$ & 0.000 & $0.000-\mathrm{i} 0.000$ \\
\hline
\end{tabular}

The third test problem is made up of a unit source at $(r, z)=(0.0,0.25)$. The Dirichlet boundary condition is also given by the free-field velocity potential that would arise from the same source. Hence the exact solution for the whole field is given by

$$
\varphi(\mathbf{p})=\frac{e^{i k r}}{4 \pi r} .
$$

The results from this test problem are given in Table 4.F.

\begin{tabular}{|c||c|c|}
\hline \multicolumn{3}{|c|}{ Table 4.F: Results from AlBEMA_T } \\
\hline point $(\mathrm{r}, \mathrm{z})$ & exact solution & numerical solution \\
\hline$(0.0,0.0)$ & $0.313+\mathrm{i} 0.058$ & $0.312+\mathrm{i} 0.059$ \\
$(0.0,0.5)$ & $0.313+\mathrm{i} 0.058$ & $0.312+\mathrm{i} 0.059$ \\
$(0.0,-0.5)$ & $0.091+\mathrm{i} 0.055$ & $0.090+\mathrm{i} 0.056$ \\
$(0.5,0.0)$ & $0.131+\mathrm{i} 0.057$ & $0.130+\mathrm{i} 0.058$ \\
\hline
\end{tabular}




\subsection{Application: Interior acoustics of a 2D car}

The application of the interior of a vehicle has often been used in demonstrating computational methods for solving the interior acoustic problem. For example in Petyt et al [68] the finite element method is used to compute the interior acoustic modes of a van. The concept of a computer-aided approach to designing a vehicle compartment with low noise properties is considered in Nefske et al [64] and Nefske and Sung [65], wherein again the finite element method is used to model the acoustic reponse within the enclosure.

More recently, the boundary element method has been used in this application. Methods for the interior modal analysis of the passenger compartment are considered in Banerjee et al [8], Coyette and Fife [24] and Jeong-Guon Ih et al [31]. As an application to demonstrate the subroutine AIBEM2, the boundary is a two-dimensional version of the vehicle interior used in Jeong-Guon [31]. A diagram of the vehicle interior is shown in Figure 4.2.

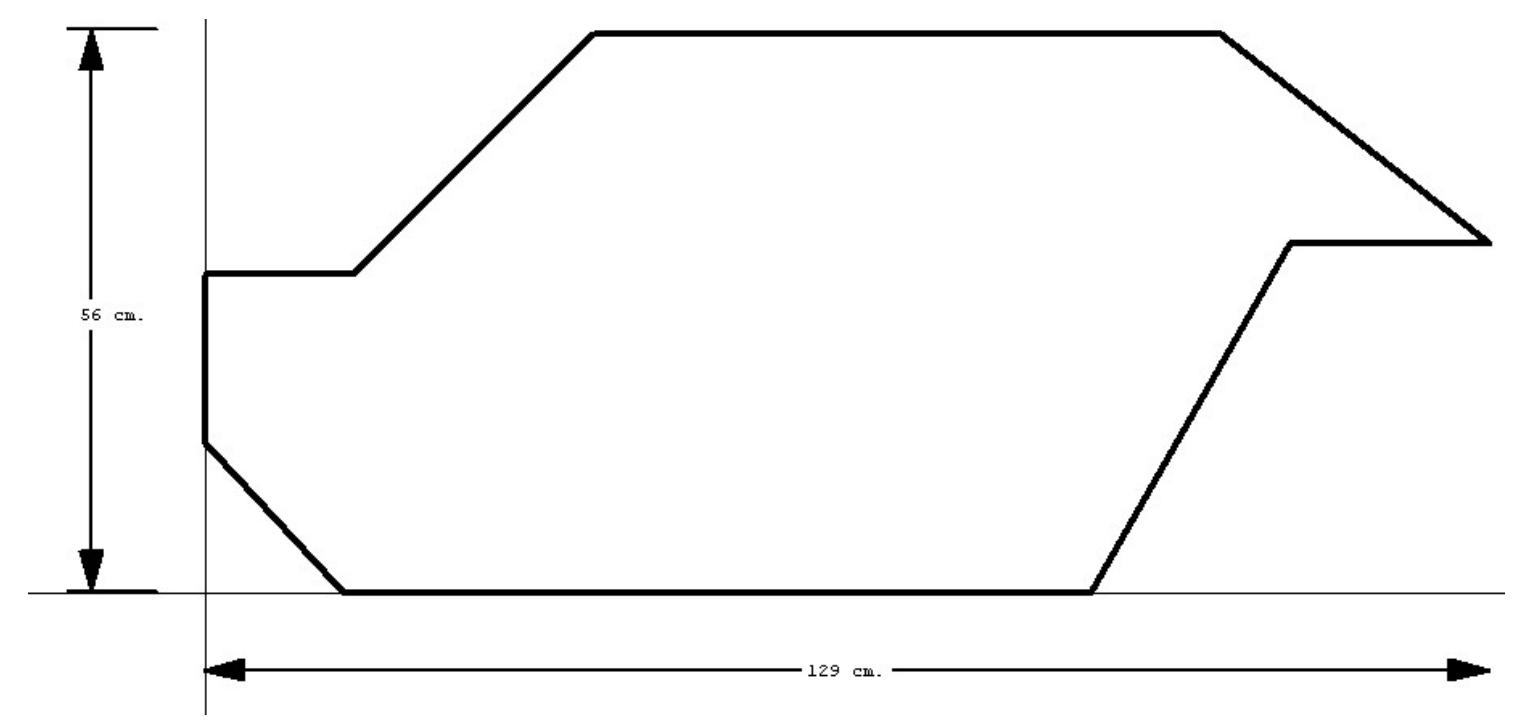

Figure 4.2. Diagram of the interior of the 2D car.

The boundary is divided into 60 elements of approximately equal size. The test is run at a range of frequencies between 0 and $1000 \mathrm{~Hz}$. The boundary condition is defined so that the diagonal part of the boundary at the driver's feet is determined to have uniform vibration of amplitude $1 \mathrm{~mm}$ at all frequencies. The remaining boundary is rigid. Figure 4.3 shows a graph of the computed sound pressure at the selected interior point $(0.5,0.4)$ in the domain. 


\subsection{Conclusion}

As stated in the introduction of Chapter 4, the solution of the interior boundary value problem by the boundary element method is well-established. The results in Tables 4.A, 4.B, 4.D, 4.E and 4.F show the accuracy of the method on the three test boundaries by comparing computed and exact solutions.

The results from the two-dimensional car show a series of peaks in the sound pressure. The peaks are the outcome of acoustic resonances. The determination of the frequencies at which the peaks occur in this type of problem is the subject of Chapter 6.

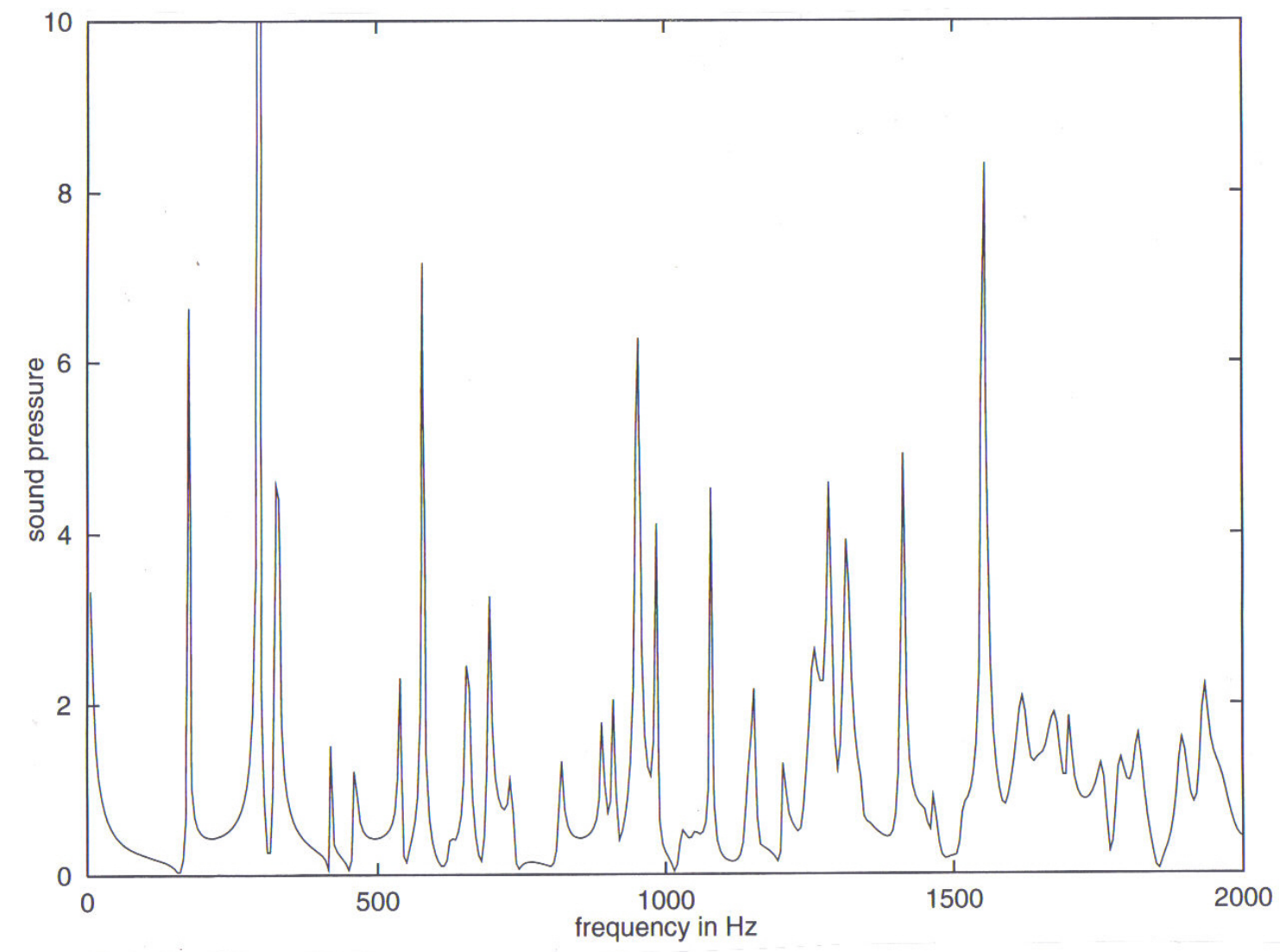

Figure 4.3. The magnitude of the sound pressure at $(0.5,0.4)$. 


\section{Chapter 5}

\section{The Exterior Acoustic Problem}

This chapter addresses the problem of computing the acoustic field in an homogeneous isotropic fluid, exterior to a closed surface or surfaces and subject to a particular boundary condition and to a specified incident acoustic field. Such problems include the determination of the sound pressure field that is produced by a radiating surface or the perturbation or scattering of an acoustic field that results from an obstruction. Let the domain of the acoustic field be the region $E$ exterior to the closed boundary $S$, as illustrated in Figure 5.1.
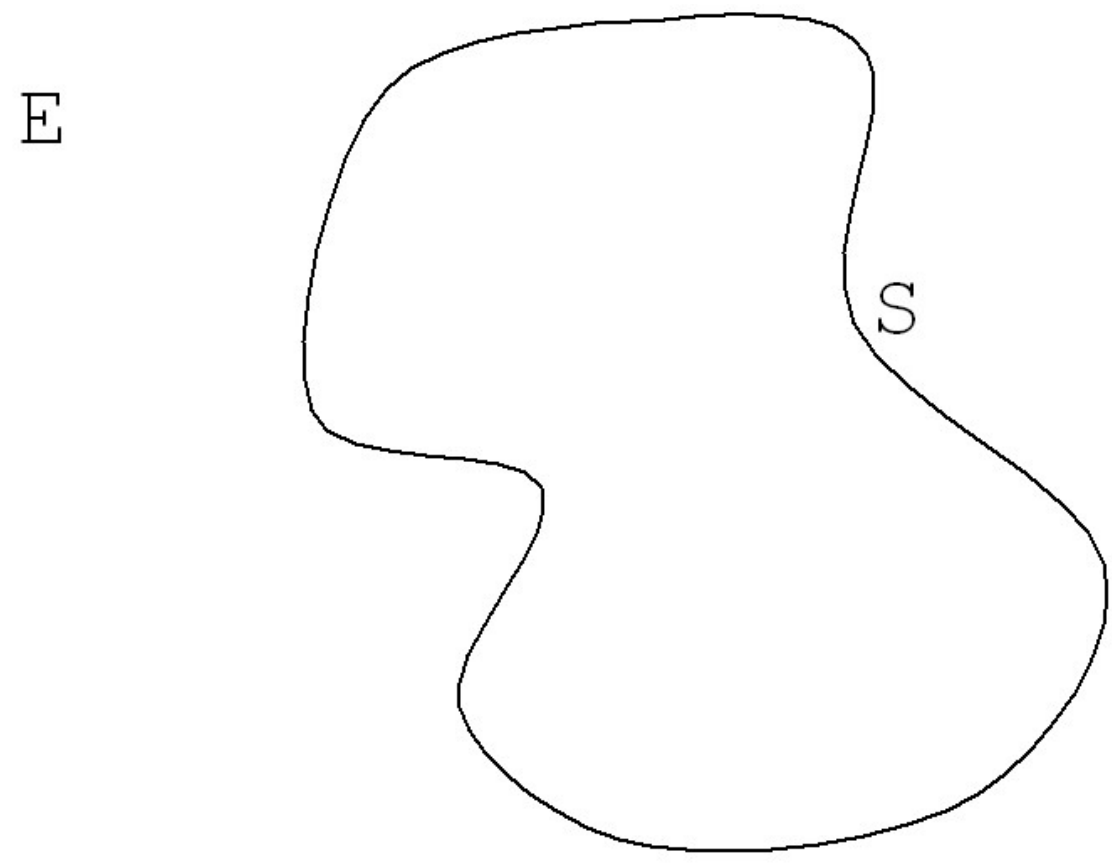

Fig 5.1. The domain of the exterior acoustic problem. 
The problem is equivalent to the solution of the Helmholtz equation

$$
\nabla^{2} \varphi(\mathbf{p})+k^{2} \varphi(\mathbf{p})=0 \quad(\mathbf{p} \in E)
$$

in the domain $E$. The exterior problem also requires a condition that the scattered and radiated waves are outgoing, this is known as the Sommerfeld radiation condition, stated earlier in Section 1.3. The boundary condition is assumed to take a general form

$$
\alpha(\mathbf{p}) \varphi(\mathbf{p})+\beta(\mathbf{p}) v(\mathbf{p})=f(\mathbf{p}) \quad(\mathbf{p} \in S)
$$

where $\alpha, \beta$ and $f$ are complex-valued functions defined on the boundary.

Although the exterior Helmholtz equation can be solved by the finite element or finite difference methods ( see, for example, Harari and Hughes [30]), such methods are clearly awkward to apply and probably inefficient since the domain is infinite. The boundary element method has an important strategic advantage over the alternative methods in that it requires discretisation of the boundary only. The solution at points in the domain can then be obtained by a straightforward integration of the boundary functions.

The application of the BEM to acoustic radiation and scattering problems has been investigated by researchers over the past three decades or so. Early contributors (see references [18], [21]) applied what may be called elementary methods; methods derived in the standard way from the integral equations arising from Green's second theorem (generally known an the Helmholtz formula of the Helmholtz-Kirchoff equation) or a single- or double-layer representation. However, the resulting BEMs, the elementary methods, were subsequently found to give unreliable results for all but a relatively low range of wavenumber. They are generally reliable for wavenumbers such that $k D<4.0$, where $D$ represents the diameter of the body or the maximum distance between any two points on the boundary - much too restrictive a condition for most applications.

There has been a large number of contributors to the research and development of boundary element methods for the solution of the exterior acoustic problem. Alternative integral formulations of the exterior Helmholtz equation were introduced in references [12], [57], [66], [54] and [15] and these will be termed the improved formulations. A further non-standard BEM, based on the original integral equation formulations but being demonstrably more reliable, was introduced by Schenck [75] and this has since become very popular since it turns out to be rather easier to implement than the methods based on the alternative formulations. Early reviews of these and other methods are given in Burton [16], [17] and Kleinmann and Roach [51]. The monograph by Amini et al [3] contains a more recent review. 
The main difficulty with the improved formulations is that they generally involve the $N_{k}$ integral operator. Although methods can be developed for its discretisation, they are notoriously difficult to devise and program. Nevertheless, as far as this work is concerned, the discretisation of all the operators has been programmed for the simple elements covered in Chapter 2. Hence the presence of the $N_{k}$ operator is no barrier to the implementation of these methods for the purposes of this work. In this Chapter the most important integral equation formulations and methods are considered. The subroutines AEBEM2, AEBEM3 and AEBEMA [33] for solving the two-, three- and axisymmetric three-dimensional problems are introduced.

\subsection{Elementary Formulations and Methods}

The elementary formulations are those that are derived from Green's second theorem or from simple layer potential representation of the solution. Although the methods are not suitable for general computation, it is important to introduce them as the alternative methods originate with these formulae.

\subsubsection{Elementary Direct Formulations}

The application of Green's second theorem to the Helmholtz equation gives the following equations:

$$
\begin{gathered}
\left\{M_{k} \varphi\right\}_{S}(\mathbf{p})-\varphi(\mathbf{p})=\left\{L_{k} v\right\}_{S}(\mathbf{p}) \quad(\mathbf{p} \in E), \\
\left\{M_{k} \varphi\right\}_{S}(\mathbf{p})-\frac{1}{2} \varphi(\mathbf{p})=\left\{L_{k} v\right\}_{S}(\mathbf{p}) \quad(\mathbf{p} \in S),
\end{gathered}
$$

where $L_{k}$ and $M_{k}$, are defined in Section 3.1 and $v(p)=\frac{\partial \varphi}{\partial n}$. The equations are also termed the Helmholtz formula or the Helmholtz-Kirchoff equation. The equation (5.3) is often also termed the surface Helmholtz equation. In this text the equations will be known as the elementary direct formulation.

The most straightforward approach to solving the exterior Helmholtz equation via the elementary formulations is to first find $\varphi$ and $v$ on the boundary from equation (5.3). The value of $\varphi(\mathbf{p})$ for points $\mathbf{p}$ in the domain can then be obtained through the discretisation of equation (5.2). 


\subsubsection{Elementary Indirect Formulations}

The elementary indirect integral equation formulations to can be obtained by writing $\varphi$ as a single- or double-layer potential;

$$
\varphi(\mathbf{p})=\left\{L_{k} \sigma_{0}\right\}_{S}(\mathbf{p}) \quad \text { or } \quad \varphi(\mathbf{p})=\left\{M_{k} \sigma_{\infty}\right\}_{S}(\mathbf{p}) \quad(\mathbf{p} \in E)
$$

where the $\sigma_{0}$ and $\sigma_{\infty}$ are source density functions defined on $S$. For points on the boundary the equations become boundary integral equations;

$$
\varphi(\mathbf{p})=\left\{L_{k} \sigma_{0}\right\}_{S}(\mathbf{p}) \quad \text { or } \quad \varphi(\mathbf{p})=\left\{\left(M_{k}+\frac{1}{2} I\right) \sigma_{\infty}\right\}_{S}(\mathbf{p}) \quad(\mathbf{p} \in S),
$$

where the jump conditions of Section 3.1 have been taken into account. In order to solve the exterior Helmholtz equation with a Dirichlet boundary condition using the above equations the first step is to solve one of the equations to obtain an approximation to either $\sigma_{0}$ or $\sigma_{\infty}$. The solution at any point $\mathbf{p}$ in the domain can then be obtained by approximating the relevant equation of (5.4).

For the Neumann problem, further equations must be derived. Differentiating the equations (5.4) with respect to $\mathbf{n}_{p}$, the normal to a point $\mathbf{p} \in S$, and taking the limit as the point approaches $S$ returns the following equations:

$$
v(\mathbf{p})=\left\{\left(M_{k}^{t}-\frac{1}{2} I\right) \sigma_{0}\right\}_{S}\left(\mathbf{p} ; \mathbf{n}_{p}\right) \quad \text { or } \quad v(\mathbf{p})=\left\{N_{k} \sigma_{\infty}\right\}_{S}\left(\mathbf{p} ; \mathbf{n}_{p}\right) \quad(\mathbf{p} \in S)
$$

The solution of one of the integral equations (5.6) returns either $\sigma_{0}$ or $\sigma_{\infty}$ and the solution in the domain can then be obtained from one of the equations (5.4).

The solution of the Robin problem by elementary indirect methods can be achieved by substituting the expressions for $\varphi$ or $v$ on $S$ into the boundary condition (5.1):

$$
\begin{aligned}
& \alpha(\mathbf{p})\left\{L_{k} \sigma_{0}\right\}_{S}(\mathbf{p})+\beta(\mathbf{p})\left\{\left(M_{k}^{t}-\frac{1}{2} I\right) \sigma_{0}\right\}_{S}\left(\mathbf{p} ; \mathbf{n}_{p}\right)=f(\mathbf{p}) \text { or } \\
& \alpha(\mathbf{p})\left\{\left(M_{k}+\frac{1}{2} I\right) \sigma_{\infty}\right\}_{S}\left(\mathbf{p} ; \mathbf{n}_{p}\right)+\beta(\mathbf{p})\left\{N_{k} \sigma_{\infty}\right\}_{S}(\mathbf{p})=f(\mathbf{p}) .
\end{aligned}
$$

Once an approximation to the solution $\sigma_{0}$ is obtained from the first of these equations or an approximation to $\sigma_{\infty}$ is obtained from the second, the solution in the domain can be obtained through approximation of the relevant equation of (5.4) .

\subsubsection{Elementary Methods}

Methods that are derived straightforwardly from the integral equations of the previous Section are termed elementary methods. A typical example is the solution of the 
exterior Neumann problem using equations (5.2), (5.3). The application of collocation to the integral equation (5.3) reduces it to the following system of equations:

$$
\left[\mathrm{M}_{k}-\frac{1}{2} \mathrm{I}\right] \underline{\hat{\varphi}}=\mathrm{L}_{k} \underline{\hat{v}}
$$

The $\mathrm{L}_{k}, \mathrm{M}_{k}$ are $n \times n$ matrices arising from the discretisation method outlined in Sections 1.1 and 2.3; for example the components of $\mathrm{L}_{k}$ are defined by $\left[\mathrm{L}_{k}\right]_{i j}=$ $\left\{L_{k} \tilde{e}\right\}_{\Delta \tilde{S}_{j}}\left(\mathbf{p}_{i}\right)$, where $\tilde{e}$ is the unit function. The vectors $\underline{\varphi}$ and $\underline{v}$ represent the values of the boundary functions $\varphi$ and $v$ at the collocation points and $\underline{\hat{\varphi}}$ and $\underline{\hat{v}}$ are their approximations. The solution at any point $\mathbf{p}$ in the domain can then be approximated using

$$
\hat{\varphi}(\mathbf{p})=\sum_{j=1}^{n}\left\{M_{k} \tilde{e}\right\}_{\Delta S_{j}}(\mathbf{p}) \hat{\varphi}_{j}-\left\{L_{k} \tilde{e}\right\}_{\Delta S_{j}}(\mathbf{p}) \hat{v}_{j},
$$

is the discrete equivalent of (5.2).

Unfortunately, such methods have been found to be very unsuitable for the general computational solution of the exterior Helmholtz equation. The underlying reason for this is that for each formulation on each boundary with a given form of boundary condition the operator over which we solve is singular for certain values of $k^{*}$, often termed the characteristic wavenumbers. For example the direct solution of the exterior Neumann problem requires the solution of equation (5.3), obtaining $\varphi$ from $v$. However at a set of real values $k^{*}$, the eigenfrequencies of the interior Dirichlet problem, the operator $M_{k^{*}}-\frac{1}{2} I$ is singular. If $k$ takes the value of any one of the values $k^{*}$ then a solution is impossible.

It might be argued that the wavenumbers for which a solution to the Helmholtz equation is sought do not coincide with any of the characteristic wavenumbers and so the problem does not arise. However, the operator over which we solve is not only singular at $k=k^{*}$ it is ill-conditioned for values of $k$ in the neighbourhood of $k^{*}$; the condition of the operator being approximately proportional to $\frac{1}{\left|k-k^{*}\right|}[4]$.

The condition of the operator over which a solution is obtained in integral equation methods is one of the most important factors governing the numerical error. In this case the numerical error can be characterised by $\frac{\epsilon}{\left|k-k^{*}\right|}$, following the profile of the condition of the operator. The $\epsilon$ is determined by the nature of the problem and the accuracy of the boundary representation and the boundary function approximation and it tends to increase gently with $k$.

Given also the fact that the characteristic wavenumbers tend to cluster more and more at higher real wavenumbers, the elementary methods are generally unsatisfactory. Reports on results of implementations of elementary methods first appeared in the 
1960s: Banaugh and Goldsmith [6], Chen and Schweikert [18], Chertock [21] and Brundrit [14]. All but the last of these references seem to have been unaware of the difficulties with the methods at the characteristic wavenumbers. The computational performance of elementary methods is compared with various alternative methods in Schenck [75], Meyer et al [60], Sayhi et al [74], for example. Because of the perceived computational difficulties, research has generally moved away from the elementary methods and towards alternative methods. A formal analysis of the solution of the exterior Helmholtz equation by elementary methods is given in Amini and Kirkup [4]. The convergence of the error with respect to element size and the accuracy of the representation of the boundary and boundary functions is considered in Juhl [38].

\subsection{The Schenck Method}

The Schenck method [75] represented an important step forward in the boundary element solution of the exterior Helmholtz equation. The method takes advantage of the complementary equation of equations (5.2) and (5.3) for points in the interior;

$$
\left\{M_{k} \varphi\right\}_{S}(\mathbf{p})=\left\{L_{k} v\right\}_{S}(\mathbf{p}) \quad(\mathbf{p} \in D)
$$

If we write the discrete form of this equation for a selected set of points $\mathbf{q}_{1}, \mathbf{q}_{2}, \ldots, \mathbf{q}_{m} \in$ $D$ then the following linear system of equations can be obtained:

$$
\overline{\mathrm{M}}_{k} \underline{\varphi} \approx \overline{\mathrm{L}}_{k} \underline{v}
$$

where $\overline{\mathrm{L}}_{k}$ is an $m \times n$ matrices with $\left[\overline{\mathrm{L}}_{k}\right]_{i j}=\left\{L_{k} \tilde{e}\right\}_{\Delta \tilde{S}_{j}}\left(\mathbf{q}_{i}\right)$ and the other matrix is defined similarly.

The matrix approximation (5.10) is regarded as a further set of $m$ approximations that relate $\underline{\varphi}$ and $\underline{v}$. Schenck suggested that instead of basing the numerical method on the equation related to (5.7) alone, the surface solution is found from the following matrix-vector approximation that is constructed from both the approximation (5.7) and (5.10):

$$
\left[\begin{array}{c}
\mathrm{M}_{k}-\frac{1}{2} \mathrm{I} \\
\overline{\mathrm{M}}_{k}
\end{array}\right] \underline{\varphi} \approx\left[\begin{array}{c}
\mathrm{L}_{k} \\
\overline{\mathrm{L}}_{k}
\end{array}\right] \underline{v},
$$

where the matrices in (5.11) have $n$ columns and $n+m$ rows.

A least squares method may be employed to find an approximation to the unknown boundary function. For example for the Neumann problem this is equivalent to finding 
the solution of the matrix-vector equation

$$
\left[\begin{array}{ll}
\left(\mathrm{M}_{k}-\frac{1}{2} \mathrm{I}\right)^{T} & \left(\overline{\mathrm{M}}_{k}\right)^{T}
\end{array}\right]\left[\begin{array}{c}
\mathrm{M}_{k}-\frac{1}{2} \mathrm{I} \\
\overline{\mathrm{M}}_{k}
\end{array}\right] \underline{\hat{\varphi}}=\left[\begin{array}{ll}
\left(\mathrm{M}_{k}-\frac{1}{2} \mathrm{I}\right)^{T} & \left(\overline{\mathrm{M}}_{k}\right)^{T}
\end{array}\right]\left[\begin{array}{c}
\mathrm{L}_{k} \\
\overline{\mathrm{L}}_{k}
\end{array}\right] \underline{v},
$$

which is arrived at through pre-multiplying both sides of the system (5.11) by the transpose of the matrix on the left hand side. Since the transposed matrix has the dimension $n \times(n+m)$ then the matrices on both sides of (5.12) are $n \times n$ when

multiplied out and the equation is simply a system of $n$ equations in $n$ unknowns that can be solved by standard methods.

For further details on the Schenck method, often also termed the CHIEF method, the reader is referred to Seybert et al [76], [77], [78] and Juhl [37], for example. The Schenck method has the potential of greatly extending the range of wavenumbers over which a solution of the exterior Helmholtz equation can be achieved when compared to the elementary methods. However, the Schenck method suffers from the difficulty that the number of interior points and their positions is not clear. At higher wavenumbers, more and more interior points are required to maintain accuracy on the one hand but the size of the matrices must correspondingly increase on the other, signalling a loss of efficiency.

The Schenck method remains popular, particularly since it avoids the necessity of discretising the $N_{k}$ operator, which is generally required by the improved formulations that are considered in the next Section.

\subsection{Improved Formulations}

The term improved formulations is used to include the integral equation representations that were introduced in the 1960s and early 1970s for which the integral operator over which a solution was sought was always non-singular. Hence the formulations were intended to form a firm foundation for the boundary element method solution of the exterior Helmholtz equation. The general drawback with these methods is the inclusion of the $N_{k}$ operator, which is very difficult to discretise. However, for the elements considered in this manual, the discrete $N_{k}$ operator is available through the subroutines outlined in Chapter 3. Hence it is feasible to implement the improved methods and, because of their robustness in comparison with the methods outlined earlier, boundary element methods that result from them are implemented in the accompanying software and considered in the next Sections. In this Chapter a particular implementation of an improved method is described and this forms the basis 
of the subroutines AEBEM2, AEBEM3 and AEBEMA that are introduced in Section 5.5 .

\subsubsection{Improved Indirect Formulation}

The equations for the improved indirect formulation that are directly applicable to the solution of the Dirichlet problem were apparently introduced independently by Brakhage and Werner [12], Leis [57] and Panich [66]. The formulation involves writing $\varphi(\mathbf{p})$ for $\mathbf{p} \in E$ as a linear sum of single- and double-layer potentials;

$$
\varphi(\mathbf{p})=\left\{\left(L_{k}+\nu M_{k}\right) \sigma_{\nu}\right\}_{S}(\mathbf{p}) \quad(\mathbf{p} \in E) .
$$

For points on the boundary the equation becomes

$$
\varphi(\mathbf{p})=\left\{\left(L_{k}+\nu\left(M_{k}-\frac{1}{2} I\right)\right) \sigma_{\nu}\right\}_{S}(\mathbf{p}) \quad(\mathbf{p} \in S) .
$$

For a given Dirichlet boundary condition the boundary integral equation (5.14) has a unique solution provided the parameter $\nu$ is such that $\operatorname{Im}(\nu) \neq 0$. Having computed $\sigma_{\nu}(\mathbf{p})$ for $\mathbf{p} \in S$, the solution in the domain can be computed through substituting it into an approximation based on (5.13).

An integral equation that is suitable for solving the exterior Neumann problem can be derived through differentiating (5.13) with respect to the outward normal to the boundary and taking the limit as $\mathbf{p}$ approaches the boundary at the base of the normal. This gives the following boundary integral equation:

$$
v(\mathbf{p})=\left\{\left(M_{k}^{t}-\frac{1}{2} I+\nu N_{k}\right) \sigma_{\nu}\right\}_{S}\left(\mathbf{p} ; \mathbf{n}_{p}\right) \quad(\mathbf{p} \in S),
$$

which is attributed to Kussmaul [54]. In correspondence with the earlier formulation (5.14), the equation (5.15) has a unique solution provided $\operatorname{Im}(\nu) \neq 0$. The solution of the Neumann problem can be obtained from finding an approximation to $\sigma_{\nu}$ by solving the boundary integral equation (5.15). The solution in the domain can then be obtained by approximating (5.13).

For the general Robin problem the boundary condition takes the form (5.1). Substituting the expressions for $\varphi(\mathbf{p})$ and $v(\mathbf{p})$ obtained earlier into the boundary condition gives the following integral equation:

$$
\left\{\left(\alpha(\mathbf{p})\left(L_{k}+\nu\left(M_{k}-\frac{1}{2} I\right)\right)+\beta(\mathbf{p})\left(M_{k}^{t}+\frac{1}{2} I+\nu N_{k}\right)\right) \sigma_{\nu}\right\}_{S}\left(\mathbf{p} ; \mathbf{n}_{p}\right)=f(\mathbf{p}) .
$$

Given a suitable Robin condition, the functions $\alpha(\mathbf{p}), \beta(\mathbf{p})$ and $f(\mathbf{p})$, an approximation to $\sigma_{\nu}(\mathbf{p})$ for $\mathbf{p} \in S$ can be obtained from the numerical solution of the integral equation (5.16). The solution at points of the domain $E$ can then be found by the substitution of the result into (5.13). 


\subsubsection{Improved Direct Formulation}

The improved direct formulation originates in the paper by Burton and Miller [15]. The formulation is a hybrid of the elementary direct formulation (5.3) and equation that arises through differentiating that equation with respect to the normal to the boundary;

$$
\left\{N_{k} \varphi\right\}_{S}\left(\mathbf{p} ; \mathbf{n}_{p}\right)=\left\{\left(M_{k}^{t}+\frac{1}{2} I\right) v\right\}_{S}\left(\mathbf{p} ; \mathbf{n}_{p}\right) \quad(\mathbf{p} \in S) .
$$

The improved direct integral equation formulation is simply a linear combination of (5.3) with this equation, giving the following:

$$
\left\{\left(M_{k}-\frac{1}{2} I+\mu N_{k}\right) \varphi\right\}_{S}\left(\mathbf{p} ; \mathbf{n}_{p}\right)=\left\{\left(L_{k}+\mu\left(M_{k}^{t}+\frac{1}{2} I\right)\right) v\right\}_{S}\left(\mathbf{p} ; \mathbf{n}_{p}\right) \quad(\mathbf{p} \in S) .
$$

The boundary integral equation can be used to solve both the Neumann and Dirichlet problems for the exterior Helmholtz equation; the numerical solution of (5.17) gives both functions $\varphi$ and $v$ on $S$ and approximation of (5.2) gives the solution at any point in the exterior. The integral equation (5.17) has a unique solution provided $\operatorname{Im}(\mu) \neq 0$ [15]. For the general Robin problem the integral equation (5.17) must be solved alongside the specified Robin condition (5.1). A method for carrying this out will be given in the next Section.

\subsubsection{Scattering}

When an incident acoustic field is modified by an obstacle the result is termed the scattered field. The concept of scattering is analagous to that of field modification considered in the previous Chapter, and the inclusion of the incident field term in the integral equation formulations is entirely similar. In scattering problems there is an incident field in the domain, termed $\varphi^{\mathrm{i}}$, which is the field that would exist if there were no boundaries, or the free-space acoustic field. Such problems can also be solved by the boundary element method, it only requires a generalisation of the integral equations and the corresponding alteration of the resulting boundary element methods.

To complete this Section the improved integral reformulations of the Helmholtz equation are generalised. The improved methods based on these formulations are given in the next Section. The generalisation of the elementary methods and the Schenck method in order to include the scattering term is not explicitly carried out in this text, although the development of such methods should be clear from the formulations given. 


\section{Indirect formulation}

The inclusion of the scattering term generalises the integral equations (5.13), (5.14), (5.15) as follows:

$$
\begin{gathered}
\varphi(\mathbf{p})=\varphi^{\mathrm{i}}(\mathbf{p})+\left\{\left(L_{k}+\nu M_{k}\right) \sigma_{\nu}\right\}_{S}(\mathbf{p}) \quad(\mathbf{p} \in E), \\
\varphi(\mathbf{p})=\varphi^{\mathrm{i}}(\mathbf{p})+\left\{\left(L_{k}+\nu\left(M_{k}+\frac{1}{2} I\right)\right) \sigma_{\nu}\right\}_{S}(\mathbf{p}) \quad(\mathbf{p} \in S), \\
v(\mathbf{p})=v^{\mathrm{i}}(\mathbf{p})+\left\{\left(M_{k}^{t}-\frac{1}{2} I+\nu N_{k}\right) \sigma_{\nu}\right\}_{S}\left(\mathbf{p} ; \mathbf{n}_{p}\right) \quad(\mathbf{p} \in S)
\end{gathered}
$$

where $v_{i}=\frac{\partial \varphi_{i}}{\partial n_{p}}$.

For a Dirichlet (Neumann) boundary condition, the solution can be found by solving (5.19) ((5.20)) to find $\sigma_{\nu}$ and then substituting the result into (5.18) to find $\varphi$ in $E$. Substituting the expressions (5.19) and (5.20) for $\varphi$ and $v$ into the equation for the more general Robin boundary condition (5.1) gives

$$
\left\{\left(\alpha ( \mathbf { p } ) \left\{\varphi^{\mathrm{i}}(\mathbf{p})+\left(L_{k}+\nu\left(M_{k}-\frac{1}{2} I\right)\right)+\beta(\mathbf{p})\left\{v^{\mathrm{i}}(\mathbf{p})+\left(M_{k}^{t}+\frac{1}{2} I+\nu N_{k}\right) \sigma_{\nu}\right\}_{S}\left(\mathbf{p} ; \mathbf{n}_{p}\right)=f(\mathbf{p}) .\right.\right.\right.
$$

\section{Direct formulation}

The solution of the exterior acoustic problem on the boundary $S$ can be determined through solving the following integral equation:

$$
\left\{\left(M_{k}-\frac{1}{2} I+\mu N_{k}\right) \varphi\right\}_{S}\left(\mathbf{p} ; \mathbf{n}_{p}\right)=-\varphi^{\mathrm{i}}(\mathbf{p})-\mu v^{\mathrm{i}}(\mathbf{p})+\left\{\left(L_{k}+\mu\left(M_{k}^{t}+\frac{1}{2} I\right)\right) v\right\}_{S}\left(\mathbf{p} ; \mathbf{n}_{p}\right)
$$

for $(\mathbf{p} \in S)$, a generalisation of equation (5.17), subject to the boundary condition (5.1). Once $\varphi(\mathbf{p})$ and $v(\mathbf{p})$ are obtained through solving the above equation, the solution in the domain can be obtained by the integration

$$
\varphi(\mathbf{p})=\varphi^{\mathrm{i}}(\mathbf{p})+\left\{M_{k} \varphi\right\}_{S}(\mathbf{p})-\left\{L_{k} v\right\}_{S}(\mathbf{p}) \quad(\mathbf{p} \in E) .
$$

\subsection{Improved Boundary Element Methods}

The first step is to approximate the boundary $S$ by a set of $n$ panels $\tilde{S}=\sum_{j=1}^{n} \Delta \tilde{S}_{j}$, as considered in Chapter 2. The integral operators are approximated by the technique outlined in Chapter 3 and the resulting linear system of equations is solved. The overall method is equivalent to the solution of the integral equations by collocation. 


\subsubsection{Improved Indirect Method}

The collocation method reduces the indirect boundary integral equations (5.19), (5.20) to the linear systems of approximations

$$
\begin{gathered}
\underline{\varphi} \approx \underline{\varphi}^{\mathrm{i}}+\left[\mathrm{L}_{k}+\nu\left(\mathrm{M}_{k}+\frac{1}{2} \mathrm{I}\right)\right] \underline{\sigma}_{\nu} \quad \text { and } \\
\underline{v} \approx \underline{v}^{\mathrm{i}}+\left[\mathrm{M}_{k}^{t}-\frac{1}{2} \mathrm{I}+\nu \mathrm{N}_{k}\right] \underline{\sigma}_{\nu} .
\end{gathered}
$$

Applying the boundary condition at the collocation points as in the direct method gives the equation

$$
\mathrm{D}_{\alpha} \underline{\varphi}+\mathrm{D}_{\beta} \underline{v}=\underline{f}
$$

where $\mathrm{D}_{\alpha}, \mathrm{D}_{\beta}$ are diagonal matrices with $\left[D_{\alpha}\right]_{i i}=\alpha\left(\mathbf{p}_{i}\right),\left[D_{\beta}\right]_{i i}=\beta\left(\mathbf{p}_{i}\right)$. Substituting the approximations for $\underline{\varphi}$ and $\underline{v}$ given above into equation (5.24) gives the following:

$$
\left[\mathrm{D}_{\alpha}\left\{\mathrm{L}_{k}+\nu\left(\mathrm{M}_{k}+\frac{1}{2} \mathrm{I}\right)\right\}+\mathrm{D}_{\beta}\left\{\mathrm{M}_{k}^{t}-\frac{1}{2} \mathrm{I}+\nu \mathrm{N}_{k}\right\}\right] \underline{\sigma}_{\nu} \approx \underline{f}-\mathrm{D}_{\alpha} \underline{\varphi}^{\mathrm{i}}-\mathrm{D}_{\beta} \underline{v}^{\mathrm{i}},
$$

which is also the discrete equivalent of (5.21).

In the indirect boundary element method, the first stage is to find the approximation ${\hat{\sigma_{\nu}}}_{\nu}$ to the representation of the source density function $\underline{\sigma_{\nu}}$. This can be done by solving the following linear system of equations

$$
\left[\mathrm{D}_{\alpha}\left\{\mathrm{L}_{k}+\nu\left(\mathrm{M}_{k}+\frac{1}{2} \mathrm{I}\right)\right\}+\mathrm{D}_{\beta}\left\{\mathrm{M}_{k}^{t}-\frac{1}{2} \mathrm{I}+\nu \mathrm{N}_{k}\right\}\right] \underline{\hat{\sigma}}_{\nu}=\underline{f}-\mathrm{D}_{\alpha} \underline{\varphi}^{\mathrm{i}}-\mathrm{D}_{\beta} \underline{v}^{\mathrm{i}} .
$$

The equation (5.25) is simply a matrix-vector equation that can be immediately solved by Gaussian elimination-type methods. Having obtained $\underline{\hat{\sigma}}_{\nu}$, the solution to (5.25), the approximate solution in the domain can be found using the discrete equivalent of (5.18):

$$
\hat{\varphi}(\mathbf{p})=\varphi^{\mathrm{i}}(\mathbf{p})+\sum_{j=1}^{n}\left(\left\{L_{k} \tilde{e}\right\}_{\Delta S_{j}}+\nu\left\{M_{k} \tilde{e}\right\}_{\Delta S_{j}}\right) \hat{\sigma}_{\nu j}
$$

\subsubsection{Improved Direct Method}

The application of collocation to the integral equation (5.22) reduces it to the following linear system of equations:

$$
\left[\mathrm{M}_{k}-\frac{1}{2} \mathrm{I}+\mu \mathrm{N}_{k}\right] \underline{\varphi} \approx-\underline{\varphi}^{\mathrm{i}}-\mu \underline{v}^{\mathrm{i}}+\left[\mathrm{L}_{k}+\mu\left(\mathrm{M}_{k}^{t}+\frac{1}{2} \mathrm{I}\right)\right] \underline{v} .
$$


The $\mathrm{L}_{k}, \mathrm{M}_{k}, \mathrm{M}_{k}^{t}$ and $\mathrm{N}_{k}$ are $n \times n$ matrices arising from the discretisation method outlined in Sections 1.2 and 3.3; for example the components of $\mathrm{L}_{k}$ are defined by $\left[\mathrm{L}_{k}\right]_{i j}=\left\{L_{k} \tilde{e}\right\}_{\Delta \tilde{S}_{j}}\left(\mathbf{p}_{i}\right)$, where $\tilde{e}$ is the unit function.

The vectors $\underline{\varphi}$ and $\underline{v}$ represent the values of the boundary functions $\varphi$ and $v$ at the collocation points. The method involves finding the solution of the linear system of equations

$$
\left[\mathrm{M}_{k}-\frac{1}{2} \mathrm{I}+\mu \mathrm{N}_{k}\right] \underline{\hat{\varphi}}=-\underline{\varphi}^{\mathrm{i}}-\mu \underline{v}^{\mathrm{i}}+\left[\mathrm{L}_{k}+\mu\left(\mathrm{M}_{k}^{t}+\frac{1}{2} \mathrm{I}\right)\right] \underline{\hat{v}}
$$

subject to the boundary condition applied at the collocation points

$$
\alpha_{i} \hat{\varphi}_{i}+\beta_{i} \hat{\underline{v}}_{i}=f_{i} \quad \text { for } \quad i=1,2, \ldots, n \quad \text { or } \quad \mathrm{D}_{\alpha} \underline{\varphi}+\mathrm{D}_{\beta} \underline{\underline{v}}=\underline{f},
$$

with $\alpha_{i}=\alpha\left(\mathbf{p}_{i}\right), \beta_{i}=\beta\left(\mathbf{p}_{i}\right)$ and the $\mathrm{D}_{\alpha}$ and $\mathrm{D}_{\beta}$ denote diagonal matrices with $\left[\mathrm{D}_{\alpha}\right]_{i i}=\alpha_{i}$ and $\left[\mathrm{D}_{\beta}\right]_{i i}=\beta_{i}$, to find $\underline{\hat{\varphi}}$ and $\underline{\hat{\hat{v}}}$, the approximations to $\underline{\varphi}$ and $\underline{\mathrm{v}}$.

In the cases of a pure Dirichlet or pure Neumann boundary condition then the equations can be solved by a standard method such as LU factorization or Gaussian elimination. However in the general case the equations (5.28) can be used to rearrange the linear system of equations (5.27) and the matrix-vector equation that arises can then be solved by standard methods. The method is carried out by subroutine CGLS used for solving systems of equations of the form (5.27), (5.28) is described in Appendix 3.

Once $\underline{\hat{\varphi}}$ and $\underline{\hat{v}}$ are obtained, equation (5.23) can be used to return an approximation to the solution at any point $\mathbf{p}$ in the domain:

$$
\varphi(\mathbf{p}) \approx \varphi^{\mathrm{i}}(\mathbf{p})+\sum_{j=1}^{n}\left\{M_{k} \tilde{e}\right\}_{\Delta S_{j}} \hat{v}_{j}-\sum_{j=1}^{n}\left\{L_{k} \tilde{e}\right\}_{\Delta S_{j}} \hat{\varphi}_{j} \hat{v}_{j}
$$

or

$$
\hat{\varphi}(\mathbf{p})=\varphi^{\mathrm{i}}(\mathbf{p})+\sum_{j=1}^{n}\left\{M_{k} \tilde{e}\right\}_{\Delta S_{j}} \hat{\varphi}_{j}-\sum_{j=1}^{n}\left\{L_{k} \tilde{e}\right\}_{\Delta S_{j}} \hat{v}_{j}
$$

\subsection{Subroutines AEBEM2, AEBEM3 and AEBEMA}

In this Section the subroutines AEBEM2, AEBEM3 and AEBEMA are introduced. The purpose of the subroutines is to solve the exterior acoustic problem. Each subroutine's parameter list has the following general form:

SUBROUTINE AEBEM $\{2$ or 3 or $\mathrm{A}(\}$

real wavenumber, description of boundary and set of exterior solution points, 
boundary condition,

incident field at boundary points and at exterior solution points, control and validation parameters, solution at boundary points and at exterior solution points (solution), working space )

The subroutines require input of the conditions of the acoustic field - the wavenumber, a geometrical description of the boundary of the domain (as covered in Chapter 2) and a list of the points in the exterior domain where a solution is sought, the boundary condition, the incident field (if any). The subroutine returns the solution at the boundary points and at the selected points in the domain. The use of the subroutines are demonstrated by the programs AEBEM2_T, AEBEM3_T and AEBEMA_T in the next Section.

\subsubsection{Solution Strategy of the AEBEM* routines}

In the AEBEM* routines the exterior Helmholtz equation is solved by the improved direct boundary element method. That is finding the solution of (5.27) subject to the discrete boundary condition (5.28). This will result in obtaining (approximations to) both $\varphi$ and $v$ on $S$. The solution in the domain is then found using equation (5.29).

A simple analysis of the magnitude of the integral operators suggests that a weighting

of the form $\mu \sim \frac{1}{k}$ tends to ensure that the relative contribution from each integral operator on either side of the equation remains in balance, whatever the value of $k$. $\mu$ is a parameter in the Fortran subroutines for solving the interior acoustic problem. In the test problems the parameter is chosen as follows:

$$
\mu=\frac{i}{k+1}
$$

\subsubsection{Subroutine AEBEM2}

Subroutine AEBEM2 computes the solution of the two-dimensional Helmholtz equation in the domain exterior to a closed boundary. The boundary $(S)$ is approximated by a set of straight line elements.

C SUBROUTINE AEBEM2(K,

C * MAXNV, NV, VERTEX, MAXNSE, NSE, SELV,

C * MAXNPE, NPE, PEXT, 


$$
\begin{array}{ll}
\mathrm{C} & * \text { SALPHA, SBETA, SF, SFFPHI, SFFVEL, PFFPHI, } \\
\mathrm{C} & * \text { LSOL, LVALID , EGEOM , MU, } \\
\mathrm{C} & * \text { SPHI, SVEL, PEPHI, } \\
\mathrm{C} & * \text { WKSPC1, WKSPC2, WKSPC3, WKSPC4, } \\
\mathrm{C} & * \text { WKSPC5, WKSPC6, WKSPC7) }
\end{array}
$$

C Wavenumber (input)

C real K: Must be positive.

C Geometry of the boundary S (input)

$C$ integer MAXNV: The limit on the number of vertices of the polygon

C that defines (approximates) S. MAXNV $>=3$.

C integer NV: The number of vertices on $\mathrm{S} .3<=\mathrm{NV}<=\mathrm{MAXNV}$.

C real VERTEX (MAXNV,2): The coordinates of the vertices. VERTEX(i,1),

C VERTEX $(i, 2)$ are the $x, y$ coordinates of the i-th vertex.

$C$ integer MAXNSE: The limit on the number of elements describing $S$.

C MAXNSE $>=3$.

$C$ integer NSE: The number of elements describing S. 3<=NSE $<=$ MAXNSE.

$C$ integer SELV(MAXNSE,2): The indices of the two vertices defining

$C$ each element. The i-th element have vertices

C (VERTEX $(\operatorname{SeLV}(i, 1), 1), \operatorname{VerTEX}(\operatorname{SELV}(i, 1), 2))$ and

C (VERTEX $(\operatorname{SELV}(i, 2), 1), \operatorname{VERTEX}(\operatorname{SeLV}(i, 2), 2))$.

C Exterior points at which the solution is to be observed (input)

$C$ integer MAXNPE: Limit on the number of points exterior to the

C boundary. MAXNPE $>=1$.

$C$ integer NPE: The number of exterior points. $0<=N P E<=$ MAXNPE.

$C$ real PEXT(MAXNPE,2). The coordinates of the exterior point.

C $\operatorname{PEXT}(i, 1), \operatorname{PEXT}(i, 2)$ are the $x, y$ coordinates of the $i$-th point.

C The boundary condition ( $\{\backslash$ alpha $\}\{\backslash$ phi $\}+\{\backslash$ beta $\} v=f$ ) (input)

C complex SALPHA (MAXNSE): The values of $\{\backslash$ alpha\} at the centres

C of the elements.

C complex SBETA(MAXNSE): The values of $\{\backslash$ beta $\}$ at the centres

$C$ of the elements.

C complex SF(MAXNSE): The values of $f$ at the centres of the

C elements. 
C complex SFFPHI (MAXNSE): The incident velocity potential at the

C centres of the elements

C complex SFFVEL(MAXNSE): The derivative of the incident velocity

C centres of the elements

C complex PFFPHI(MAXNPE): The incident velocity potential at the chosen

C exterior points

C Validation and control parameters (input)

C logical LSOL: A switch to control whether the particular

C solution is required.

C logical LVALID: A switch to enable the choice of checking of

C subroutine parameters.

C real EGEOM: The maximum absolute error in the parameters that

C describe the geometry.

C complex MU: The weighting parameter in the direct formulations.

C As a default, set $\mathrm{MU}=\mathrm{I} /(\mathrm{K}+1)$.

C Solution (output)

C complex SPHI(MAXNSE): The velocity potential ( $\backslash$ phi\}) at the

C centres of the boundary elements.

C complex SVEL(MAXNSE): The velocity ( $v$ or $d\{\backslash p h i\} / d n$ where $n$

$C$ is the outward normal to the boundary) at the centres of the

C boundary elements.

C complex PEPHI(MAXNPE): The velocity potential ( $\backslash$ phi\}) at the

C exterior points.

C Working space

C complex WKSPC1 (MAXNSE, MAXNSE)

C complex WKSPC2 (MAXNSE, MAXNSE)

C complex WKSPC3(MAXNPE, MAXNSE)

C complex WKSPC4 (MAXNPE, MAXNSE)

C complex WKSPC5 (MAXNSE, MAXNSE)

C complex WKSPC6 (MAXNSE, MAXNSE)

C logical WKSPC7(MAXNSE) 


\subsubsection{Subroutine AEBEM3}

Subroutine AEBEM3 computes solution of the three-dimensional Helmholtz equation in the domain exterior to a closed surface. The boundary $(S)$ is approximated by a set of planar triangular elements.

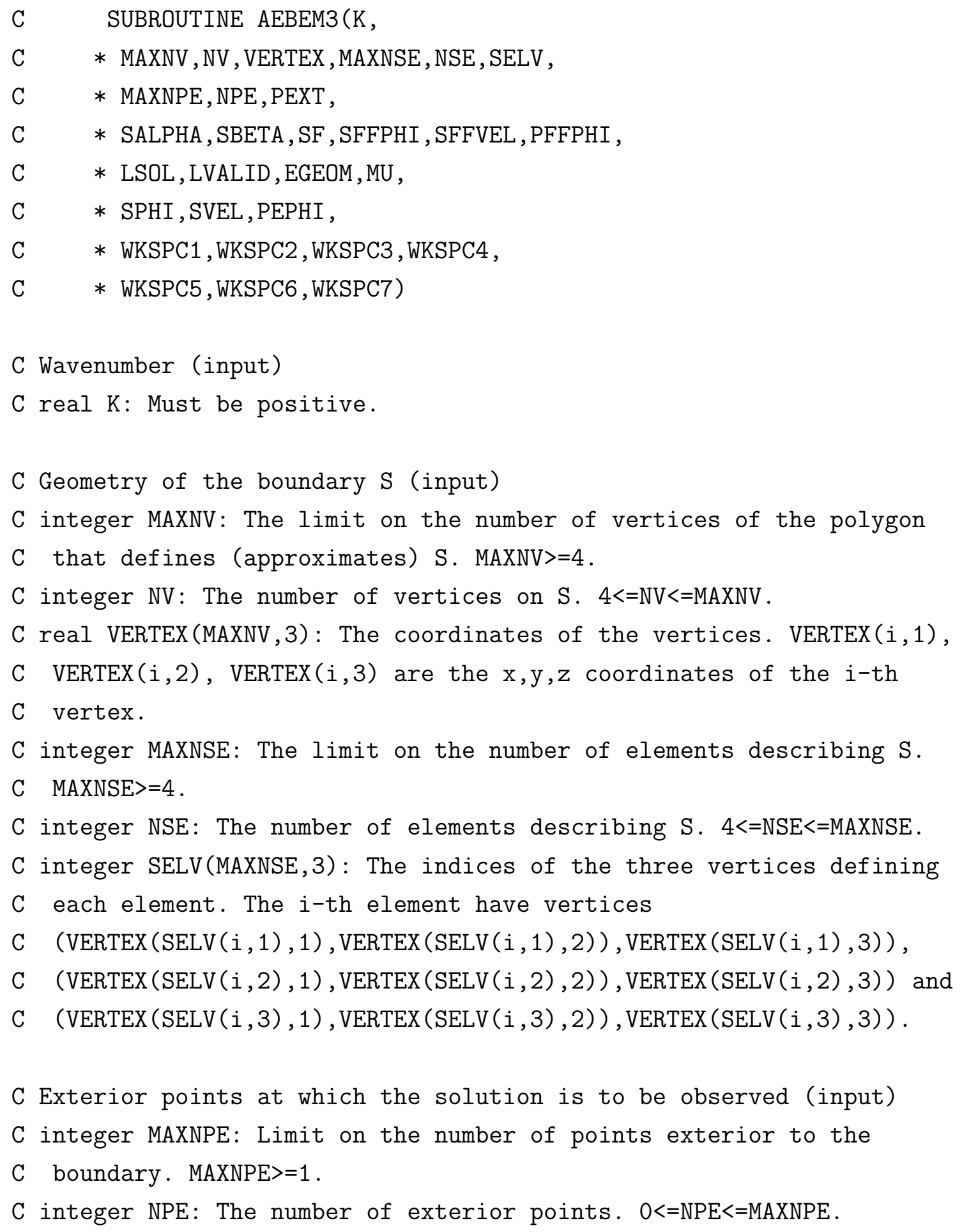


C real PEXT(MAXNPE,3). The coordinates of the exterior point.

C $\operatorname{PEXT}(i, 1), \operatorname{PEXT}(i, 2), \operatorname{PEXT}(i, 3)$ are the $x, y, z$ coordinates of the $i-t h$

C point.

C The boundary condition ( $\{\backslash$ alpha $\}$ phi $+\{\backslash$ beta $\} V=f$ ) (input)

C complex SALPHA(MAXNSE): The values of $\{\backslash$ alpha\} at the centres

$C$ of the elements.

C complex SBETA(MAXNSE): The values of $\{\backslash$ beta $\}$ at the centres

$\mathrm{C}$ of the elements.

C complex SF(MAXNSE): The values of $f$ at the centres of the

C elements.

C complex SFFPHI(MAXNSE): The incident velocity potential at the

C centres of the elements

C complex SFFVEL(MAXNSE): The derivative of the incident velocity

$C$ centres of the elements

C complex PFFPHI(MAXNPE): The incident velocity potential at the chosen $\mathrm{C}$ exterior points

C Validation and control parameters (input)

C logical LSOL: A switch to control whether the particular solution is C required.

C logical LVALID: A switch to enable the choice of checking of

C subroutine parameters.

C real EGEOM: The maximum absolute error in the parameters that

$C$ describe the geometry.

C complex MU: The weighting parameter in the direct formulations.

C As a default, set $M U=I /(K+1)$.

C Solution (output)

C complex SPHI(MAXNSE): The velocity potential ( $\backslash$ phi $\}$ ) at the

$C$ centres of the boundary elements.

C complex SVEL(MAXNSE): The velocity ( $\mathrm{v}$ or $\mathrm{d}\{\backslash \mathrm{phi}\} / \mathrm{dn}$ where $\mathrm{n}$ is

$C$ the outward normal to the boundary) at the centres of the boundary

C elements.

C complex PEPHI(MAXNPE): The velocity potential (\{\phi\}) at the

C exterior points. 


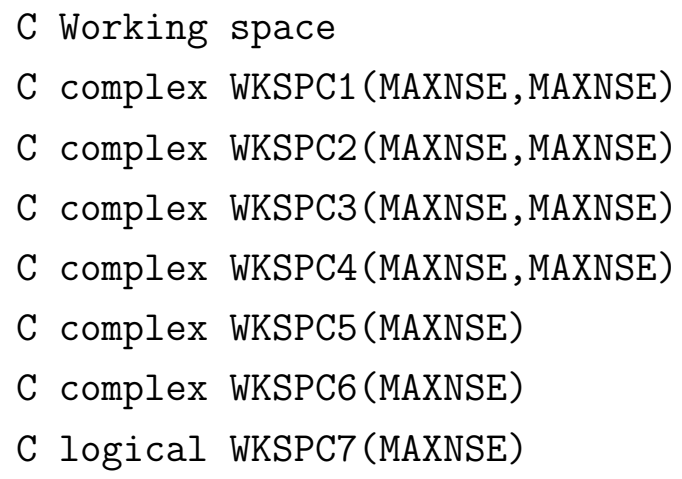

The subroutine parameters that specify the exterior three-dimensional Helmholtz problem must be set up in the main program. Let this be called MAIN.FOR. The following files must be linked together to construct the complete program:

$M A I N . F O R$ (and files containing any user-defined sub-programs), AEBEM3.FOR,

H3LC.FOR, the file for computing the discrete operators - see Chapter 3,

CGLS.FOR, the file for computing the solution to a linear system - see Appendix 3, GEOM3D.FOR, the file for 3D geometry - see Appendix 6.

\subsubsection{Subroutine AEBEMA}

Subroutine AEBEMA computes solutions of the axisymmetric three-dimensional Helmholtz equation in the domain exterior to a closed surface. The boundary $(S)$ is approximated by a set of conical elements.

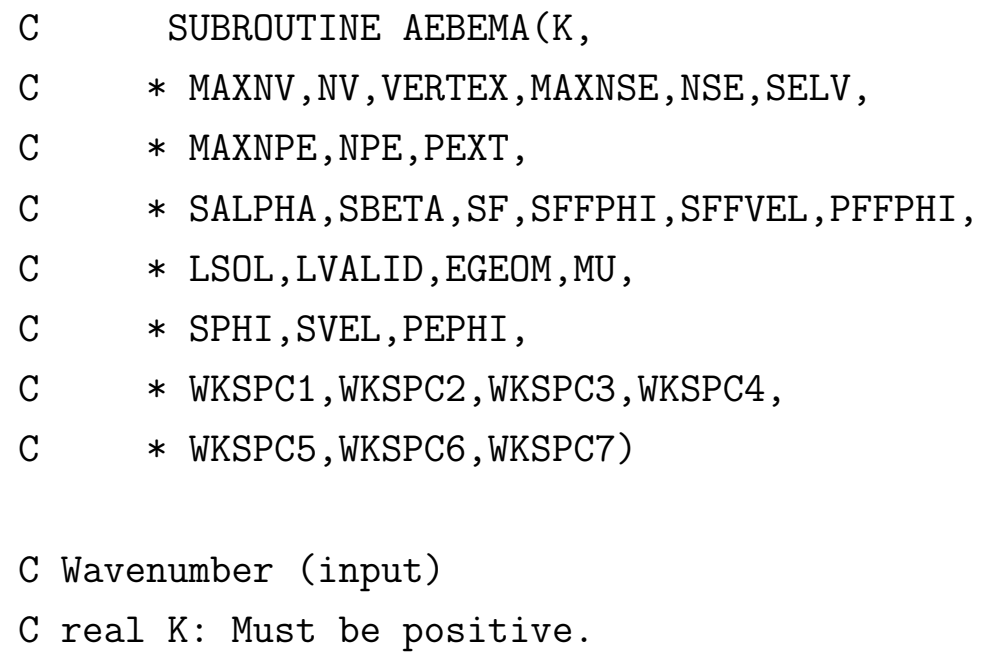


C Geometry of the boundary S (input)

$C$ integer MAXNV: The limit on the number of vertices of the generator

C that defines (approximates) $S$. MAXNV $>=3$.

$C$ integer NV: The number of vertices on $\mathrm{S} .3<=\mathrm{NV}<=\mathrm{MAXNV}$.

$C$ real VERTEX (MAXNV,2): The coordinates of the vertices of the

C generator. $\operatorname{VERTEX}(i, 1), \operatorname{VERTEX}(i, 2)$ are the $r, z$ coordinates of the

C i-th vertex.

$C$ integer MAXNSE: The limit on the number of elements describing $\mathrm{S}$.

C MAXNSE $>=3$.

$C$ integer NSE: The number of elements describing S. 3<=NSE<=MAXNSE.

$C$ integer SELV(MAXNSE,2): The indices of the two vertices defining

$\mathrm{C}$ each element. The generator of the $i$-th element has vertices with

C $r, z$ coordinates (VerteX $(\operatorname{SeLV}(i, 1), 1), \operatorname{VerteX}(\operatorname{SelV}(i, 1), 2))$ and

C (VerteX $(\operatorname{SELV}(i, 2), 1), \operatorname{VerTEX}(\operatorname{SELV}(i, 2), 2))$.

C Exterior points at which the solution is to be observed (input)

$C$ integer MAXNPE: Limit on the number of points exterior to the

C boundary. MAXNPE $>=1$.

$C$ integer NPE: The number of exterior points. $0<=N P E<=M A X N P E$.

$C$ real PEXT(MAXNPE,2). The coordinates of the exterior point.

C $\operatorname{PEXT}(i, 1), \operatorname{PEXT}(i, 2)$ are the $r, z$ coordinates of the $i$-th point.

C The boundary condition ( $\{\backslash$ alpha $\}\{\backslash p h i\}+\{\backslash$ beta $\} \mathrm{v}=f$ ) (input)

C complex SALPHA(MAXNSE): The values of $\{\backslash$ alpha\} at the centres

$C$ of the generator of the elements.

C complex SBETA(MAXNSE): The values of $\{\backslash$ beta $\}$ at the centres

$C$ of the generator of the elements.

C complex SF(MAXNSE): The values of $f$ at the centres of the

$\mathrm{C}$ of the generator of the elements.

C complex SFFPHI(MAXNSE): The incident velocity potential at the

$C$ centres of the generator of the elements

C complex SFFVEL(MAXNSE): The derivative of the incident velocity

$C$ centres of the generator of the elements

C complex PFFPHI(MAXNPE): The incident velocity potential at the chosen C exterior points 


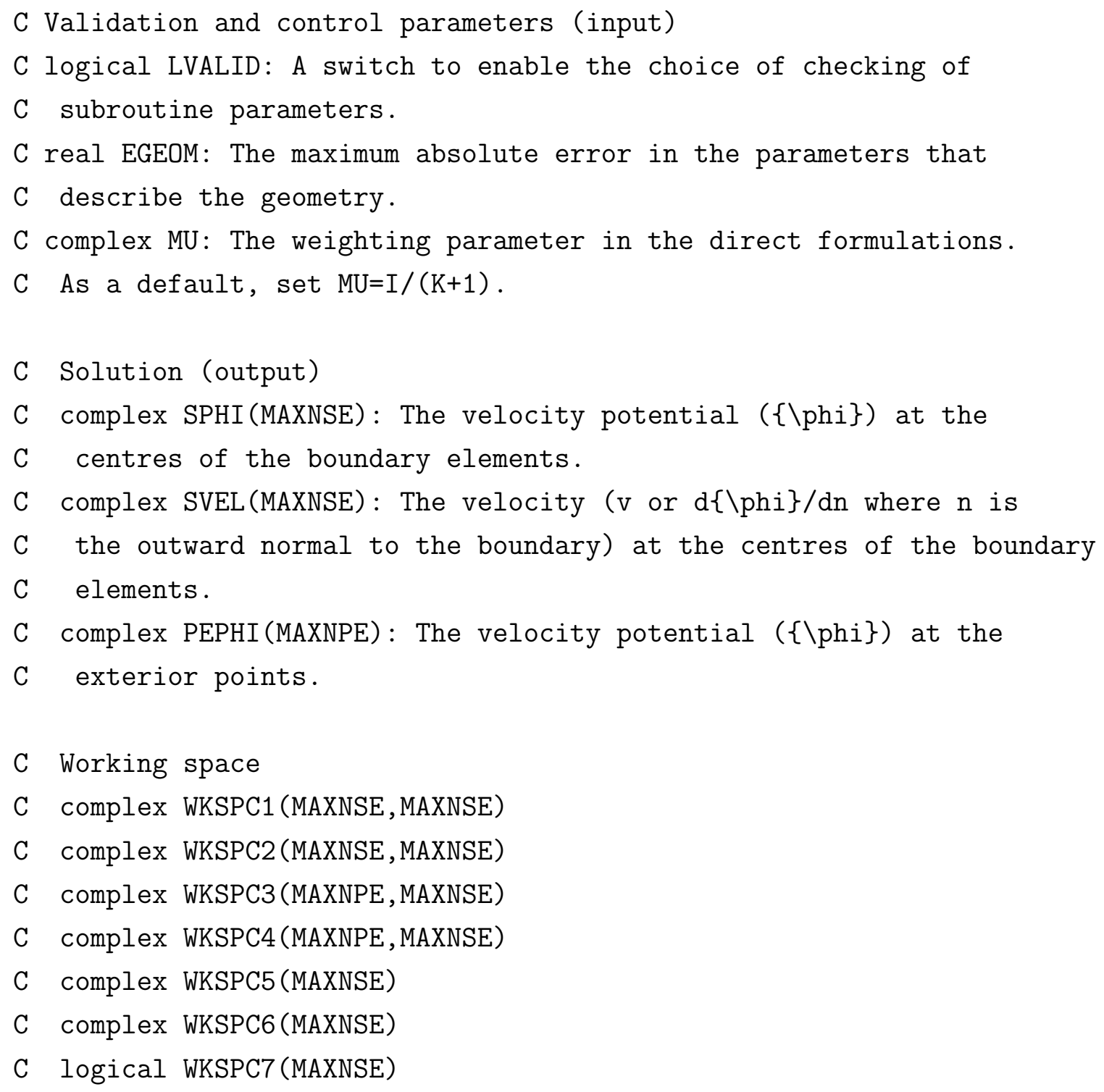

The subroutine parameters that specify the exterior three-dimensional axisymmetric Helmholtz problem must be set up in the main program. Let this be called MAIN.FOR. The following files must be linked together to construct the complete program:

MAIN.FOR (and files containing any user-defined sub-programs), AIBEMA.FOR, H3ALC.FOR, the file for computing the discrete operators - see Chapter 3, CGLS.FOR, the file for computing the solution to a linear system - see Appendix 3, GEOM2D.FOR, the file for 2D geometry - see Appendix 6, GEOM3D.FOR, the file for 3D geometry - see Appendix 6. 


\subsection{Test Programs}

In this Section the subroutines AEBEM2, AEBEM3 and AIBEMA are demonstrated through invoking them and comparing the results with analytic solutions. The corresponding main programs are AEBEM2_T, AEBEM3_T and AEBEMA_T.

\subsubsection{Program AEBEM2_T}

The main program AEBEM2_T tests module AEBEM2, the subroutine for computing the solution of the Helmholtz equation exterior to a closed boundary in a twodimensional domain. In AEBEM2_T the domain lies exterior to a square of side $0.1 \mathrm{~m}$ with the boundary represented by 32 elements. The representation of the boundary is described in Section 2.2 by the data structures VERTEX and SELV, see Tables 2.A and 2.B and Figure 2.2. Full results are given in file AEBEM2.OUT.

The acoustic medium is air at 20 celcius and 1 atmosphere so the speed of sound is $344 \mathrm{~m} / \mathrm{s}$. The chosen frequency of the test is $400 \mathrm{~Hz}$, hence $k=7.31$. For the first two tests, the velocity potential defined by $\varphi(\mathbf{p})=\frac{i}{4} H_{0}(k r)$, with $r$ being the distance from the point $\mathbf{p}$ to the centre of the square, is clearly a solution of the Helmholtz equation in the exterior domain. The boundary velocity is given by differentiation this expression for $\varphi(\mathbf{p})$ with respect to the normal to the boundary at $\mathbf{p}$ for each collocation point $\mathbf{p}$ on $S$.

In the first two tests the Dirichlet and Neumann boundary conditions arising from this potential is processed by AEBEM2. The solution is given at the points $(0,0.15)$, $(0.05,0.15),(0.1,0.15)$ and $(0.05,-0.1)$. Comparisons between the exact and numerical solutions are given in Table 5.A.

\begin{tabular}{|c||c|c|c|}
\hline \multicolumn{4}{|c|}{ Table 5.A: Results from AEBEM2_T } \\
\hline point & exact solution & $\begin{array}{c}\text { numerical solution } \\
\text { to Dirichlet condition }\end{array}$ & $\begin{array}{c}\text { numerical solution } \\
\text { to Neumann condition }\end{array}$ \\
\hline$(0.000,0.150)$ & $0.0176+\mathrm{i} 0.2100$ & $0.0198+\mathrm{i} 0.2079$ & $0.0181+\mathrm{i} 0.2104$ \\
$(0.050,0.150)$ & $0.0394+\mathrm{i} 0.2177$ & $0.0415+\mathrm{i} 0.2154$ & $0.0397+\mathrm{i} 0.2181$ \\
$(0.100,0.150)$ & $0.0176+\mathrm{i} 0.2100$ & $0.0198+\mathrm{i} 0.2079$ & $0.0181+\mathrm{i} 0.2104$ \\
$(0.050,-0.100)$ & $-0.0398+\mathrm{i} 0.1804$ & $-0.0375+\mathrm{i} 0.1792$ & $-0.0396+\mathrm{i} 0.1808$ \\
\hline
\end{tabular}




\subsubsection{Program AEBEM3_T}

The main program AEBEM3_T tests module AEBEM3, the subroutine for computing the solution of the Helmholtz equation exterior to a general closed surface in a threedimensional domain. In AEBEM3_T the domain is the exterior of a sphere of side $1 \mathrm{~m}$ with the boundary represented by 36 elements. The representation of the boundary is described fully in Section 2.3 by the data structures VERTEX and SELV, see Tables 2.C and 2.D. Full results are given in file AEBEM3.OUT.

The acoustic medium is air at 20 celcius and 1 atmosphere so that the speed of sound is $c=344 \mathrm{~m} / \mathrm{s}$. In the first two test problems the acoustic field in the exterior is defined to be

$$
\varphi=\frac{e^{j k r}}{r}
$$

which is a multiple of the Green's function and hence is clearly a solution of the Helmholtz equation. The acoustic frequency is $100 \mathrm{~Hz}$, hence $k=1.8265$. The numerical and exact solutions at the points $(0,0,2),(0,0,4),(0,0,8)$ and $(0,0,-2)$ are given in Table 5.B.

\begin{tabular}{|c||c|c|c|}
\hline \multicolumn{4}{|c|}{ Table 5.B: Results from AEBEM3_T } \\
\hline point & exact solution & $\begin{array}{c}\text { numerical solution } \\
\text { to Dirichlet condition }\end{array}$ & $\begin{array}{c}\text { numerical solution } \\
\text { to Neumann condition }\end{array}$ \\
\hline$(0,0,2)$ & $-0.4360-\mathrm{i} 0.2447$ & $-0.4628-\mathrm{i} 0.1897$ & $-0.5011-\mathrm{i} 0.2389$ \\
$(0,0,4)$ & $0.1302+\mathrm{i} 0.2133$ & $0.1557+\mathrm{i} 0.1960$ & $0.1614+\mathrm{i} 0.2274$ \\
$(0,0,8)$ & $-0.0572+\mathrm{i} 0.1112$ & $-0.0431+\mathrm{i} 0.1175$ & $-0.0549+\mathrm{i} 0.1284$ \\
$(0,0,-2)$ & $-0.4360-\mathrm{i} 0.2447$ & $-0.4628-\mathrm{i} 0.1897$ & $-0.5011-\mathrm{i} 0.2389$ \\
\hline
\end{tabular}

In this example a comparison of the computed and exact sound pressures are given. The magnitudes (in decibels) and the phases (in degrees) are given in Table 5.C. The values are computed from the sound pressures in line with the methods described in Section 1.3.

\begin{tabular}{|c||c|c|c|}
\hline \multicolumn{4}{|c|}{ Table 5.C: Results from AIBEM3_T } \\
\hline point & exact solution & $\begin{array}{c}\text { numerical solution } \\
\text { to Dirichlet condition }\end{array}$ & $\begin{array}{c}\text { numerical solution } \\
\text { to Neumann condition }\end{array}$ \\
\hline$(0,0,2)$ & $72.8 \mathrm{~dB},-60.7^{\circ}$ & $72.8 \mathrm{~dB},-69.8^{\circ}$ & $73.2 \mathrm{~dB},-64.7^{\circ}$ \\
$(0,0,4)$ & $69.8 \mathrm{~dB}, 148.6^{\circ}$ & $69.8 \mathrm{~dB}, 139.5^{\circ}$ & $70.2 \mathrm{~dB}, 144.4^{\circ}$ \\
$(0,0,8)$ & $66.8 \mathrm{~dB},-152.8^{\circ}$ & $66.8 \mathrm{~dB},-161.9^{\circ}$ & $67.2 \mathrm{~dB},-157.1^{\circ}$ \\
$(0,0,-2)$ & $72.8 \mathrm{~dB},-60.7^{\circ}$ & $72.8 \mathrm{~dB},-69.8^{\circ}$ & $73.2 \mathrm{~dB},-64.7^{\circ}$ \\
\hline
\end{tabular}


The program AEBEMA_T also shows results from a sphere scattering a point source where in the first case the potential on the sphere is assigned the incident potential in a Dirichlet condition, so that the sphere is effectively invisible. In the second case the sphere represents a rigid (non-vibrating) acoustically hard scatterer. An application similar to these examples is given in the next Section.

\subsubsection{Program AEBEMA_T}

The main program AEBEMA_T tests module AEBEMA. In AEBEMA_T the acoustic domain is exterior to a sphere of unit radius. The surface is represented by eighteen truncated conical elements, as described in Section 2.4. The acoustic medium is air at 20 celcius $(\mathrm{c}=344 \mathrm{~m} / \mathrm{s})$. Full results are given in file AEBEMA.OUT.

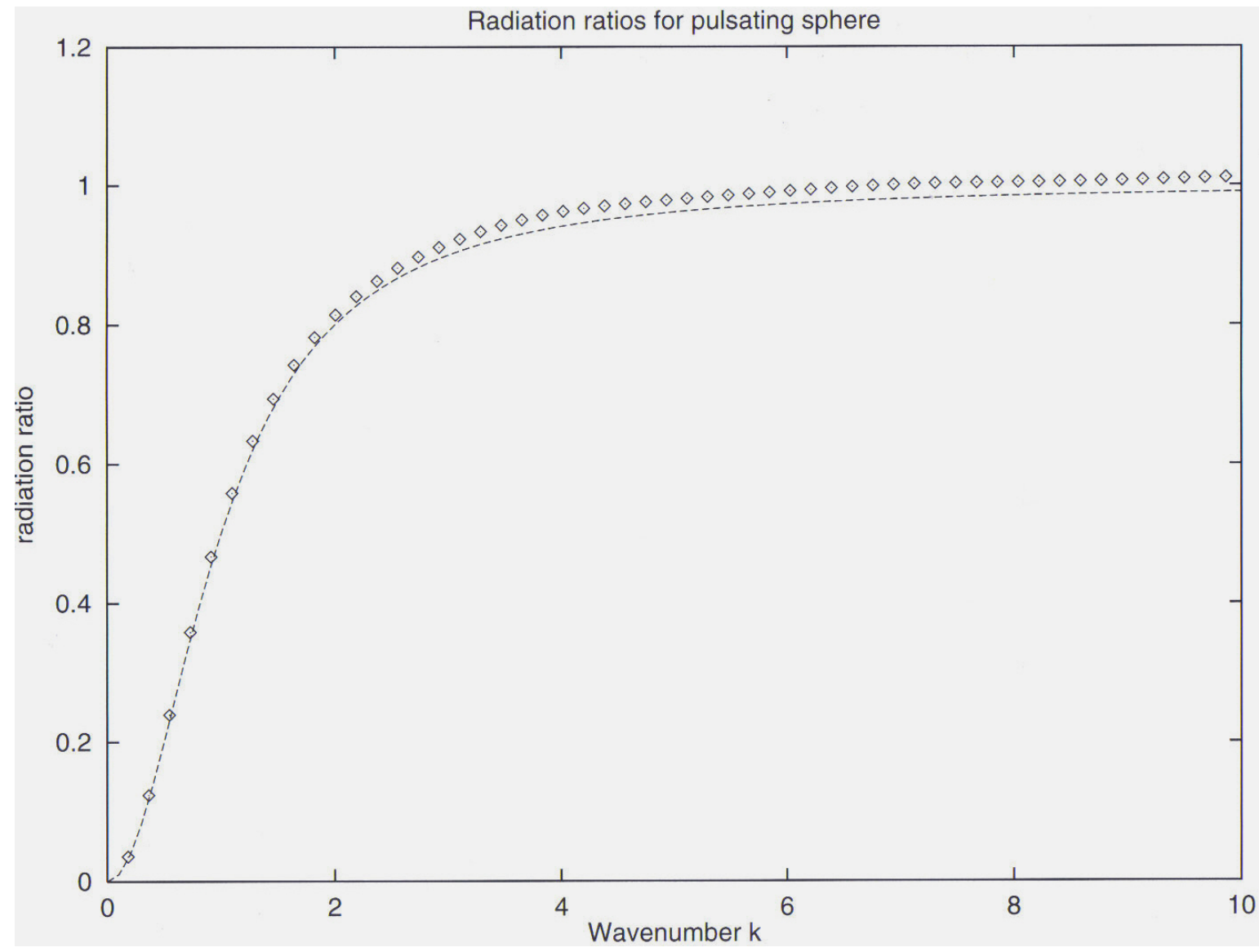

Fig 5.2. Radiation ratio curve for pulsating sphere (- exact, $\diamond$ computed).

In each test problem the frequency ranges from $10 \mathrm{~Hz}$ to $1000 \mathrm{~Hz}$ in $10 \mathrm{~Hz}$ steps. The purpose of the first test problem is to plot the radiation ratio of a pulsating 
sphere. In the test problem the surface velocity is prescribed the value of unity on each element. Note that the radiation ratio refers to the shape of the boundary condition, the amplitude is arbitrary. The results from the test are directed to the file AEBEMA.OUT. The exact radiation ratio for a sphere of radius unity, pulsating at wavenumber $k$ is $\frac{k^{2}}{k^{2}+1}$. A comparison of the computed and exact radiation ratios are given in Figure 5.2.

Results for an oscillating sphere, that is the sphere vibrating up and down without changing volume, are given in Figure 5.3. These results can be obtained from a test problem similar to AEBEMA by setting the same Neumann surface condition but with SFVAL(ITEST, ISP $)=$ SELCNT $($ ISP, 2). The exact radiation ratio of an oscillating sphere is $\frac{k^{4}}{4+k^{4}}$.

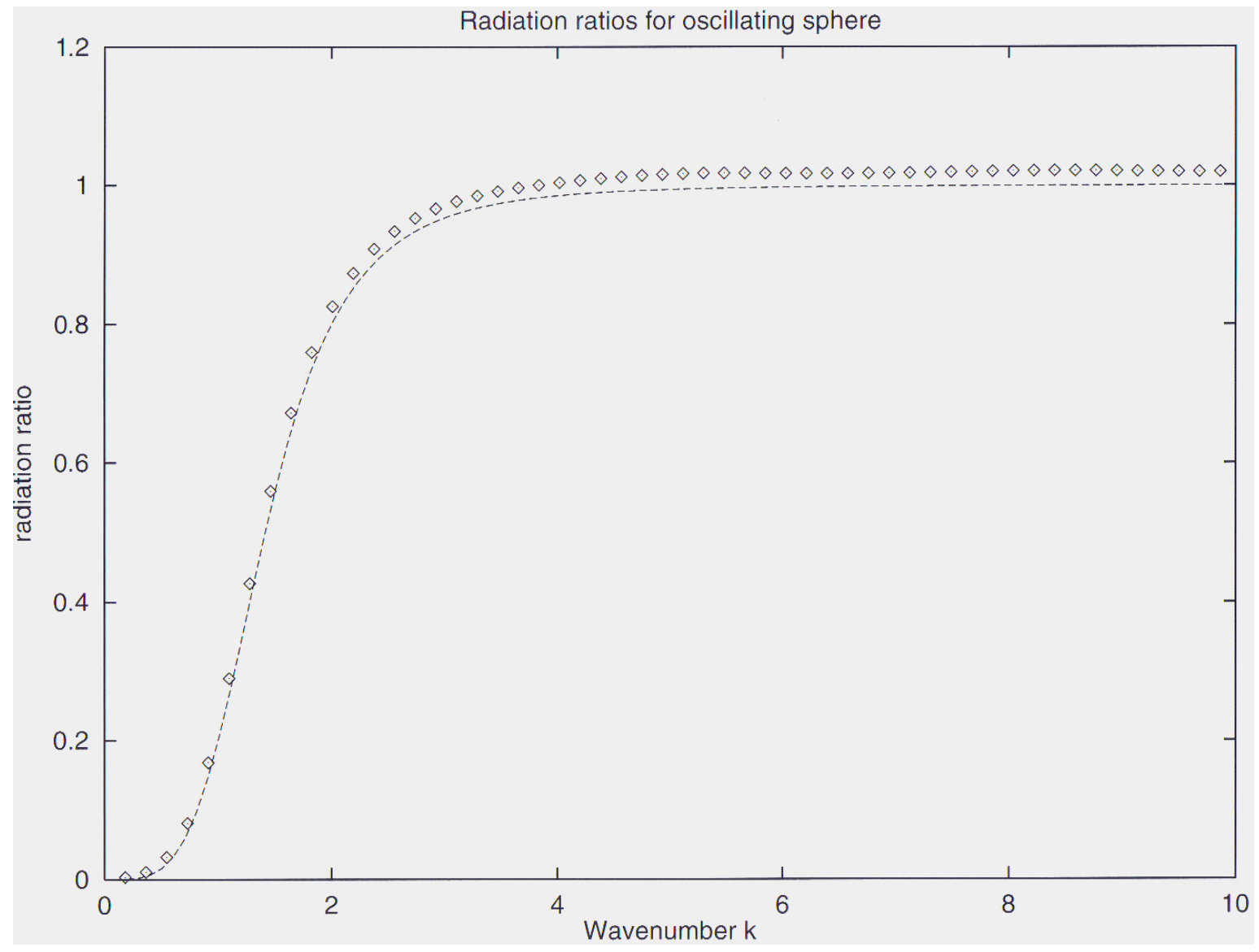

Fig 5.3. Radiation ratio curve for oscillating sphere (- exact, $\diamond$ computed). 
www.boundary-element-method.com

\subsection{Application: Engine Noise Analysis}

A computational prediction of the acoustic properties of an engine design can be useful. It enables the engineer to analyse the noise output of an engine at the early design stage and hence make the necessary adjustments. This Section is on the prediction of the noise output from an engine block. In order to do this the engine is modelled as an arbitrary three-dimensional vibrating surface radiating into free-space.

From the theoretical point of view, the BEM can closely represent the the physical situation of the engine in free-space or in an anechoic chamber. In order to apply the method, the surface of the block must be simplified so some of the surface details need to be omitted. The boundary element method in this application has been considered by a number of researchers, for example references [52], [76].

For this type of problem subroutine AEBEM3 is most suitable. However, the results presented in this Section were obtained using a prototype program that uses similar elements and method but preceded AEBEM3 by a number of years. The work of this Section was originally published in reference [45] and the reader is advised to consult that paper if further details are required.

\subsubsection{Details}

The velocity distribution (at each frequency) on the surface, required for the input of the Neumann boundary condition, is determined through using accelerometers fitted at a set number of points over the surface. In order to apply the boundary element method the surface is simplified and represented by around 550 planar triangular elements with the vertices of the triangles generally being at the accelerometer points. On each boundary element the surface velocity is determined by averaging the values of the surface velocity at the three vertices. At vertices where there was no accelerometer reading the velocity was prescribed a zero value.

\subsubsection{Results}

The sound power was computed at a range of frequencies from $400 \mathrm{~Hz}$ to $2400 \mathrm{~Hz}$. The results of this are compared with measured results in Figure 5.4. The measured results are found by integrating the readings from a microphone array; a method based on equation (1.22). The boundary element mesh of the rig, showing the computed surface intensity pattern at $1120 \mathrm{~Hz}$, is shown on the cover of this textbook. The 
colours range from deep blue on the areas of low intensity through green, yellow, orange red and to purple on the areas of high intensity. Only the middle-left cylinder was excited in the test and this is reflected in the results.

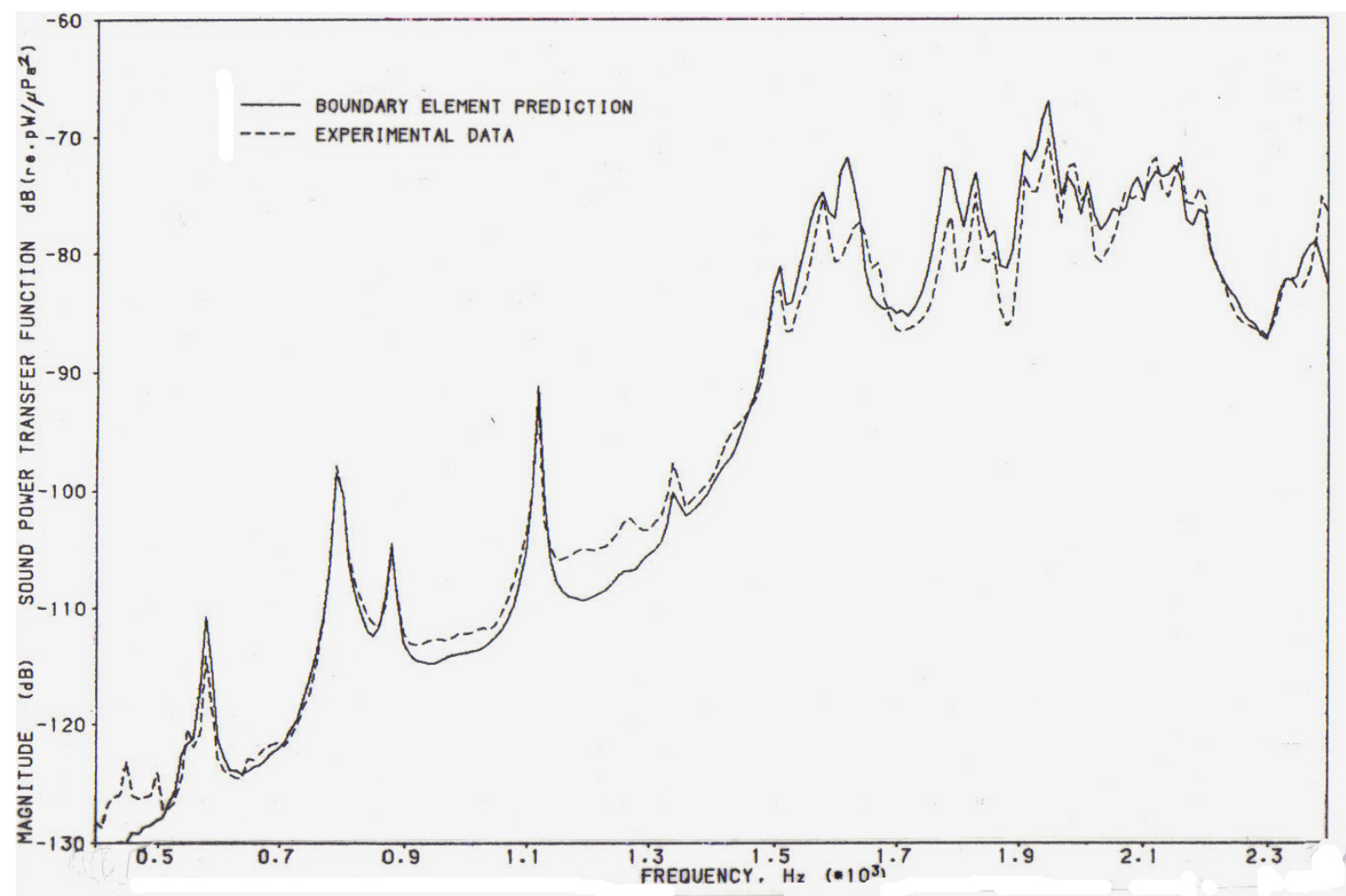

Fig 5.4. Comparison between computed and measured sound powers.

\subsection{Conclusion}

Table 5.A shows the results from computing the potentials exterior to a square. The results from Table 5.B show similar results for the exterior to a sphere. The noticeably less-accurate results in Table 5.B can be attributed to the crude approximation that is made to the sphere. In Table 5.C, the results of Table 5.B are repeated but the sound pressures are given in terms of decibels and phases. This demonstrates another important way of interpreting the results from the subroutines and also shows that often the numerical error appears far less significant when it is viewed in decibel units.

In the final test problem the important acoustic property of the radiation ratio is considered. In Figures 5.1 and 5.2 the computed versus exact radiation ratios for pulsating and oscillating spheres across a wide wavenumber range are given where 
the acoustic field is taken to be axisymmetric. The results show some numerical drift from the exact solution with increasing wavenumber. As a general rule, the accuracy of the BEM solution of acoustic problems slowly deteriorates with frequency if the same boundary element mesh is used throughout. It may often be necessary to use finer meshes at higher wavenumbers.

In the introduction to the Chapter, a history of the difficulties in employing the boundary element method successfully to exterior problems was outlined. However, the test problems in Section 5.6 and the application in Section 5.7 show that the improved formulations provide the foundation for methods that are robust throughout the frequency range; the methods do not show the wild errors that have been reported in the solution by the elementary methods of Section 5.1.

The Schenck or CHIEF method of Section 5.2 is currently the most popular in the solution of exterior acoustic problems. However it is the author's view that the method, however implemented, will tend to degrade at higher wavenumbers, although the point at which this happens is often beyond the range of wavenumbers of interest in practice. Basing the boundary element method on the improved formulations of Burton and Miller [15] (direct) and Brackage and Werner [12], Leis [57], Panich [66] and Kussmaul [54] (indirect) is the most suitable for the numerical solution of exterior acoustic problems. 


\section{Chapter 6}

\section{Interior Modal Analysis}

In this Chapter it is shown how the boundary element method can be used to obtain the resonant frequencies and the mode shapes of an enclosed homogeneous isotropic fluid; the computational solution of the interior Helmholtz eigenvalue problem. The problem is that of finding the values of the wavenumber $k$ and a non-trivial scalar function $\varphi$ such that the Helmholtz equation

$$
\nabla^{2} \varphi(\mathbf{p})+k^{2} \varphi(\mathbf{p})=0 \quad(\mathbf{p} \in D)
$$

is satisfied in an interior domain $D$ with boundary $S$ and subject to a homogeneous boundary condition of the form

$$
\alpha(\mathbf{p}) \varphi(\mathbf{p})+\beta(\mathbf{p}) \frac{\partial \varphi(\mathbf{p})}{\partial n_{p}}=0 \quad(\mathbf{p} \in S)
$$

where $\alpha(\mathbf{p})$ and $\beta(\mathbf{p})$ are known complex-valued functions of $\mathbf{p}(\in S)$ and $\mathbf{n}_{p}$ is the unit outward normal to the boundary at $\mathbf{p}$. The non-trivial solutions $k=k^{*}$ and $\varphi(\mathbf{p})=$ $\varphi^{*}(\mathbf{p}) \quad(\mathbf{p} \in D \cup S)$ are termed the characteristic wavenumbers and eigenfunctions and they are dependent on the boundary $S$ and the boundary functions $\alpha(\mathbf{p})$ and $\beta(\mathbf{p})$. The characteristic wavenumbers are all real numbers and they correspond to the resonant frequencies of the enclosed fluid. The eigenfunctions are equivalent to the mode shapes.

The Helmholtz eigenvalue problem is amenable to solution via finite element or finite difference methods. In these cases, the problem reduces to that of solving a generalised linear eigenvalue problem of the form

$$
\left(\mathrm{K}-k^{2} \mathrm{M}\right) \underline{x}=\underline{0}
$$

where the matrices $\mathrm{K}$ and $\mathrm{M}$ (termed the stiffness and mass matrices) in (6.3) are sparse and structured and are independent of $k$. Standard computational algorithms 
are available for solving generalised linear eigenvalue problems. Indeed special techniques (such as iterative methods) are available for solving the general problem (6.3), given the special structure of the matrices and the fact that only a fraction of the full set of eigenvalues are generally required [69]. Hence eigenfrequency analysis of the Helmholtz problem via the finite element or finite difference method is straightforward.

In cases where it is applicable, it is well known that the boundary element method has an important advantage over the finite element and finite difference methods: the partial differential equation governing the domain is reduced to an integral equation relating values of $\varphi$ and $\frac{\partial \varphi}{\partial n}$ on the boundary only. Hence the dimension of the problem is effectively reduced by one. However, the application of the boundary element method reduces the Helmholtz eigenvalue problem to that of solving an eigenproblem of the form

$$
\mathrm{A}_{k} \underline{\mu}=\underline{0}
$$

where the matrix $A_{k}$ is generally full, having no particular structure but with each component being a continuously differentiable complex-valued function of $k$.

Because of the main advantage of the boundary element method over finite element and finite difference methods stated earlier, the matrix in (6.4) is generally much smaller than the matrices in (6.3), for any given modal analysis problem and a given level of required accuracy. The disadvantages of this approach are that the eigenvalue problem (6.4) is non-linear and the components of the $\mathrm{A}_{k}$ matrix are defined in terms of integrals and hence may be costly to evaluate. The solution of non-linear eigenvalue problems are considered in references [55], [73] and [84]. Unfortunately, standard algorithms for solving non-linear eigenvalue problems are not generally available.

The problem of solving the Helmholtz eigenvalue problem via boundary element-type methods have been given some consideration by researchers. For example iterative methods such as the secant method are applied to the problem of finding the roots of the equation $\operatorname{det}\left(\mathrm{A}_{k}\right)=0$ in references [81], [27] and [1]. However, this is not a satisfactory method when the matrix $\mathrm{A}_{k}$ is large [84]. A similar method, based on finding the values of $k$ for which the smallest eigenvalues of $A_{k}$ is zero is considered in [58]. Unfortunately, these methods are unwieldy since they do not compute the solutions simultaneously; they require a starting point to be chosen for each required eigenfrequency.

In reference [8] a hybrid of the boundary element and finite element method is introduced. The method seems to have the advantage of the finite element method in that a linear eigenvalue problem results and the advantage of the boundary element 
method in that a solution on the boundary only is sought in the main computation. The method is considered further in references [24], [2].

In general, both eigenfrequencies and eigenfunctions of the Helmholtz problem will be of interest The method considered in this Chapter was introduced in Kirkup and Amini [46]. The method involves approximating each component of the matrix $\mathrm{A}_{k}$ by a polynomial in $k$ in some given sub-range of the full wavenumber range. This allows us to re-write the non-linear eigenvalue problem (6.4) in the form of a standard generalised eigenvalue problem. Thus all of the eigenvalues in the sub-range are computed simultaneously.

\subsection{Formulations of the Eigenvalue Problem}

The integral equation formulations of the interior Helmholtz equation were given in Section 4.1. The distinction here is that the boundary condition (6.2) is homogeneous and the characteristic wavenumbers $k^{*}$ and the eigenfunctions $\varphi^{*}$ are sought, rather than the solution of a boundary-value problem.

\subsubsection{Indirect Formulation}

An indirect formulation of the interior Helmholtz problem is derived by writing $\varphi$ as a single-layer potential, as first shown in Section 4.1. The following equations result:

$$
\begin{gathered}
\varphi(\mathbf{p})=\left\{L_{k} \sigma\right\}_{S}(\mathbf{p}) \quad(\mathbf{p} \in D \cup S), \\
v(\mathbf{p})=\left\{M_{k}^{t} \sigma\right\}_{S}\left(\mathbf{p} ; \mathbf{n}_{p}\right)+\frac{1}{2} \sigma(\mathbf{p}) \quad(\mathbf{p} \in S),
\end{gathered}
$$

where $\sigma$ is a source density function defined on $S$, (termed $\sigma_{0}$ in Section 4.1). Also $v(\mathbf{p})=\frac{\partial \varphi(\mathbf{p})}{\partial n_{p}}$ and $\mathbf{n}_{p}$ is the unit outward normal to the boundary at $\mathbf{p}$.

The indirect formulations for the Dirichlet and Neumann eigenproblems are as follows:

$$
\begin{gathered}
\left\{L_{k} \mu\right\}_{S}(\mathbf{p})=0 \quad(\mathbf{p} \in S), \text { for the Dirichlet boundary condition and } \\
\left\{\left(M_{k}^{t}+\frac{1}{2} I\right) \mu\right\}_{S}\left(\mathbf{p} ; \mathbf{n}_{p}\right) \quad(\mathbf{p} \in S) \text { for the Neumann boundary condition. }
\end{gathered}
$$

For the Helmholtz eigenvalue problem with the general boundary condition (6.2) the integral equation formulation is as follows:

$$
\alpha(\mathbf{p})\left\{L_{k} \mu\right\}_{S}(\mathbf{p})+\beta(\mathbf{p})\left\{\left(M_{k}^{t}+\frac{1}{2} I\right) \mu\right\}_{S}(\mathbf{p})=0 \quad(\mathbf{p} \in S)
$$


which arises through the substitution of (6.5) and (6.6) into (6.2) where $\sigma$ has been replaced by $\mu$ in (6.5)-(6.6).

The general solution strategy is to find the solutions $k^{*}, \mu^{*}$ of the relevant integral equation defined on $S$. Each of these may then be substituted into equation (6.5) to obtain the solution at the domain points.

\subsubsection{Direct Formulation}

As in Section 4.1, the direct formulation is obtained through the application of Green's second theorem to the Helmholtz equation and can be presented as follows:

$$
\begin{gathered}
\left\{M_{k} \varphi\right\}_{S}(\mathbf{p})+\varphi(\mathbf{p})=\left\{L_{k} v\right\}_{S}(\mathbf{p}) \quad(\mathbf{p} \in D) \\
\left\{M_{k} \varphi\right\}_{S}(\mathbf{p})+\frac{1}{2} \varphi(\mathbf{p})=\left\{L_{k} v\right\}_{S}(\mathbf{p}) \quad(\mathbf{p} \in S)
\end{gathered}
$$

The direct formulation for the Dirichlet and Neumann eigenproblems are as follows:

$$
\begin{gathered}
\left\{L_{k} \mu\right\}_{S}(\mathbf{p})=0 \quad(\mathbf{p} \in S) \quad \text { for the Dirichlet boundary condition, } \\
\left\{\left(M_{k}+\frac{1}{2} I\right) \mu\right\}_{S}(\mathbf{p})=0 \quad(\mathbf{p} \in S) \quad \text { for the Neumann boundary condition, }
\end{gathered}
$$

where $\varphi$ has been replaced by $\mu$ in (6.8)-(6.9).

For the more general Robin condition (6.2) the eigenproblem cannot be written so concisely; it is the solution of (6.9) subject to the boundary condition (6.2).

\subsection{Application of Collocation}

By approximating the operators in the boundary integral equations in the way described in Section 3.3, they can each be reduced to an eigenvalue problem of the form (6.4). The first step is to approximate the boundary $S$ by a set of $n$ panels $\tilde{S}=\sum_{j=1}^{n} \Delta \tilde{S}_{j}$, as described in Chapter 2. The integral operators are approximated by the technique outlined in Chapter 3.

\subsubsection{Indirect Method}

For example the application of collocation to indirect integral equations gives an eigenvalue problem of the form (6.7) with $\mathrm{A}_{k}=\mathrm{L}_{k}$ for the Dirichlet boundary condition and $\mathrm{A}_{k}=\mathrm{M}_{k}^{t}+\frac{1}{2} \mathrm{I}$ for the Neumann boundary condition. For the more general 
Robin condition (6.2) the eigenvalue problem is of the form (6.4) with

$$
\mathrm{A}_{k}=\mathrm{D}_{\alpha} \mathrm{L}_{k}+\mathrm{D}_{\beta}\left(\mathrm{M}_{k}^{t}+\frac{1}{2} \mathrm{I}\right)
$$

where the matrices $\mathrm{L}_{k}$ and $\mathrm{M}_{k}$ are as defined by the techniques employed in Section 1.1 and Chapter $3, \mathrm{D}_{\alpha}, \mathrm{D}_{\beta}$ are diagonal matrices with $\left[\mathrm{D}_{\alpha}\right]_{i i}=\alpha\left(\mathbf{p}_{i}\right),\left[\mathrm{D}_{\beta}\right]_{i i}=\beta\left(\mathbf{p}_{i}\right)$ and the $\mathbf{p}_{i}$ are the collocation points.

\subsubsection{Direct Method}

For the Dirichlet boundary condition the discrete equivalent is identical to that of the indirect method stated earlier; $\mathrm{A}_{k}=\mathrm{L}_{k}$. For the Neumann problem and $\mathrm{A}_{k}=$ $\mathrm{M}_{k}+\frac{1}{2} \mathrm{I}$. For the more general Robin condition (6.2) the eigenvalue problem cannot be straightforwardly put in the form (6.4). It can be written in the form

$$
\left(\mathrm{M}_{k}+\frac{1}{2} \mathrm{I}\right) \underline{\varphi}=\mathrm{L}_{k} \underline{v}
$$

with

$$
\mathrm{D}_{\alpha} \underline{\varphi}+\mathrm{D}_{\beta} \underline{v}=\underline{0} .
$$

After rearrangement, these equations can take the form (6.4).

\subsection{Solution of the Non-linear Eigenvalue Problem}

The discrete eigenvalue problems are each of the form (6.4). The method employed for solving the general problem (6.4) requires that in an interval $\left[k_{A}, k_{B}\right]$ of values of $k$ the matrix $\mathrm{A}_{k}$ is approximated by a matrix polynomial in $k$

$$
\mathrm{A}_{k} \approx \mathrm{A}_{[0]}+k \mathrm{~A}_{[1]}+\ldots+k^{m} \mathrm{~A}_{[m]} \text { for } k \text { real. }
$$

The non-linear eigenvalue problem (6.4) can be replaced with the following eigenvalue problem:

$$
\left[\mathrm{A}_{[0]}+k \mathrm{~A}_{[1]}+\ldots+k^{m} \mathrm{~A}_{[m]}\right] \underline{\mu}=\underline{0} .
$$

The solutions of (6.13) are the same as those of the following generalised linear eigenvalue problem:

$$
\left[\begin{array}{ccccccc}
\mathrm{A}_{[0]} & \mathrm{A}_{[1]} & \mathrm{A}_{[2]} & . & . & \mathrm{A}_{[m-2]} & \mathrm{A}_{[m-1]} \\
0 & \mathrm{I} & 0 & . & . & 0 & 0 \\
: & : & : & : & : & : & : \\
0 & 0 & 0 & . & . & \mathrm{I} & 0 \\
0 & 0 & 0 & . & . & 0 & \mathrm{I}
\end{array}\right]\left[\begin{array}{c}
\underline{\mu} \\
k \underline{\mu} \\
: \\
k^{m-2} \underline{\mu} \\
k^{m-1} \underline{\mu}
\end{array}\right]
$$




$$
=k\left[\begin{array}{ccccccc}
0 & 0 & 0 & . & . & 0 & -\mathrm{A}_{[m]} \\
\mathrm{I} & 0 & 0 & . & . & 0 & 0 \\
: & : & : & : & : & : & : \\
0 & 0 & 0 & . & . & 0 & 0 \\
0 & 0 & 0 & . & . & \mathrm{I} & 0
\end{array}\right]\left[\begin{array}{c}
\underline{\mu} \\
k \underline{\mu} \\
: \\
k^{m-2} \underline{\mu} \\
k^{m-1} \underline{\mu}
\end{array}\right] .
$$

Equation (6.14) is amenable to solution by the QZ algorithm [61], which is available on a number of numerical libraries. Methods for solving problems of the form (6.13) are considered in references [67], [55], [28] and [84].

Since the eigenvalues $k^{*}$ of the underlying Helmholtz problem are all real and we are interested only in positive values, it is sufficient to compute the interpolant (6.12) for the positive real numbers $k$. However, as a result of numerical error, the computed wavenumber and mode shape will have small imaginary parts.

The generalised eigenvalue problem (6.14) will generally have $m \times n$ solutions. Half of these can be immediately discounted since the eigenvalues occur in pairs $\hat{k},-\hat{k}$. The full set of solutions will contain approximations to the true eigenvalues of the underlying Helmholtz problem. However, many spurious solutions are generally produced as a result of the collocation method and approximation (6.12). These spurious eigenvalues do not have small imaginary parts and hence they can be sorted from the true eigenvalues. Approximations to the true eigenvalues lying outside the range $\left[k_{A}, k_{B}\right]$ may also be produced. These approximations will generally be poor and they can be excluded from the results.

Let $\hat{k}, \hat{\mu}$ be a typical non-spurious solution to (6.14). The eigenvalue $\hat{k}$ is an approximation to the eigenfrequencies $k^{*}$ of the Helmholtz problem. The approximation to the eigenfunctions in $D \cup S$ can be recovered through the substitution of the approximation $\underline{\hat{\mu}}$ for $\sigma$ in equation (6.5) or $\underline{\hat{\mu}}$ for $\varphi$ (Dirichlet) or $\frac{\partial \varphi}{\partial n}$ (Neumann) in equations $(6.8)-(6.9)$.

\subsection{Subroutines MBEM2, MBEM3 and MBEMA}

In this Section the subroutines MBEM2, MBEM3 and MBEMA are introduced. The purpose of the subroutines is to solve the interior acoustic modal analysis problem. Each subroutine's parameter list has the following general form:

SUBROUTINE MBEM $\{2$ or 3 or $\mathrm{A}(\}$

real wavenumber, description of boundary and set of interior solution points, 
homogeneous boundary condition, validation parameters,

characteristic wavenumbers and their corresponding mode shape at boundary points and at interior solution points (solution), working space )

The subroutines require input a geometrical description of the boundary of the domain (as covered in Chapter 2) and a list of the points in the interior (from which the values of the $\varphi$ at those points allow us to construct the mode shapes), the homogeneous boundary condition, the subroutine returns the characteristic wavenumbers and their corresponding mode shape at boundary points and at interior solution points. The use of the subroutines are demonstrated by the programs MBEM2_T, MBEM3_T and MBEMA_T in the next Section.

\subsubsection{Solution Strategy of the MBEM* routines}

In the MBEM* routines the interior Helmholtz eigenvalue problem is solved by the direct boundary element method. The discrete homogeneous boundary condition (6.11) is used to rearrange the system of equations (6.10). This results in the generalised eigenvalue problem of the form (6.4) which is solved by the method described in section 6.3.

In the subroutines MBEM2, MBEM3 and MBEMA, the technique employed for deriving the polynomial approximation (6.12) involves computing $A_{k}$ at the $m+1$ Chebyshev ( $\infty$ norm) interpolation points for any selected wavenumber range $\left[k_{A}, k_{B}\right]$. The coefficient matrices $A_{[0]}, A_{[1]}, \ldots, A_{[m]}$ in (6.12) are obtained through Newton's divided differences using the value of $A_{k}$ at the selected values of $k$ in $\left[k_{A}, k_{B}\right]$.

\subsubsection{Subroutine MBEM2}

Subroutine MBEM2 computes the modal solutions of the two-dimensional Helmholtz equation in the domain interior to a closed boundary and in a predetermined frequency range. The boundary $(S)$ is approximated by a set of straight line elements.

\begin{tabular}{|c|c|c|}
\hline $\mathrm{C}$ & \multicolumn{2}{|c|}{ SUBROUTINE MBEM2 (KA, KB, MAXNK, NK, } \\
\hline $\mathrm{C}$ & * & MAXNEIG, \\
\hline $\mathrm{C}$ & $*$ & MAXNV, NV , VERTEX , MAXNSE, NSE , SELV, \\
\hline $\mathrm{C}$ & * & MAXNPI , NPI , PINT, \\
\hline
\end{tabular}




$\begin{array}{ll}\mathrm{C} & * \\ \mathrm{C} & * \\ \mathrm{C} & * \\ \mathrm{C} & * \\ \mathrm{C} & * \\ \mathrm{C} & * \\ \mathrm{C} & *\end{array}$

SALPHA, SBETA, LVALID , EGEOM, NEIG , EIGVAL , SPHI , SVEL , PIPHI, WKSPC1, WKSPC2 , WKSPC3, WKSPC4, WKSPC5, WKSPC6, WKSPC7, WKSP00, WKSP01, WKSP02, WKSP03, WKSP04, WKSP05, WKSP06, WKSP07, WKSP08, WKSP09, WKSP10, WKSP11, WKSP12)

C Wavenumber interpolation information (input)

$\mathrm{C}$ Lower limit of k-range

C REAL*8 KA

C Upper limit of k-range

C REAL*8 KB

C Limit on the number of interpolation points in the k-range

C INTEGER MAXNK

C Number of interpolation points in the $\mathrm{k}$-range

C INTEGER NK

C Geometry of the boundary S (input)

$C$ integer MAXNV: The limit on the number of vertices of the polygon

C that defines (approximates) S. MAXNV $>=3$.

$\mathrm{C}$ integer NV: The number of vertices on $\mathrm{S} .3<=\mathrm{NV}<=\mathrm{MAXNV}$.

C real VERTEX(MAXNV,2): The coordinates of the vertices. $\operatorname{VERTEX}(i, 1)$,

$C \operatorname{VERTEX}(i, 2)$ are the $\mathrm{x}, \mathrm{y}$ coordinates of the $i$-th vertex.

$C$ integer MAXNSE: The limit on the number of elements describing $S$.

C MAXNSE $>=3$.

$C$ integer NSE: The number of elements describing S. 3<=NSE<=MAXNSE.

$C$ integer SELV(MAXNSE,2): The indices of the two vertices defining

$\mathrm{C}$ each element. The i-th element have vertices

C $(\operatorname{VerteX}(\operatorname{SelV}(i, 1), 1), \operatorname{Vertex}(\operatorname{SelV}(i, 1), 2))$ and

C $(\operatorname{Vertex}(\operatorname{SelV}(i, 2), 1), \operatorname{Vertex}(\operatorname{SelV}(i, 2), 2))$.

C Interior points at which the solution is to be observed (input)

$C$ integer MAXNPI: Limit on the number of points interior to the

C boundary. MAXNPI $>=1$.

$C$ integer NPI: The number of interior points. $0<=N P I<=M A X N P I$. 
C real PINT(MAXNPI,2). The coordinates of the interior point.

C PINT $(i, 1), \operatorname{PINT}(i, 2)$ are the $\mathrm{x}, \mathrm{y}$ coordinates of the $i$-th point.

C The boundary condition ( $\{\backslash$ alpha $\}\{\backslash$ phi $\}+\{\backslash$ beta $\} \mathrm{v}=0$ ) (input)

C complex SALPHA(MAXNSE): The values of $\{\backslash$ alpha\} at the centres

$\mathrm{C}$ of the elements.

C complex SBETA(MAXNSE): The values of $\{\backslash$ beta $\}$ at the centres

$\mathrm{C}$ of the elements.

C Validation and control parameters (input)

C logical LVALID: A switch to enable the choice of checking of

C subroutine parameters.

C real EGEOM: The maximum absolute error in the parameters that

C describe the geometry.

C Solution (output)

$C$ integer NEIG: The number of eigenvalues.

C complex EIGVAL(MAXNEIG): The eigenvalues.

C complex SPHI(MAXNEIG, MAXNSE): The velocity potential ( $\{\backslash$ phi $\}$ ) at

C the centres of the boundary elements.

C complex SVEL(MAXNEIG,MAXNSE): The velocity ( $v$ or $d\{\backslash p h i\} / d n$

$\mathrm{C}$ where $\mathrm{n}$ is the outward normal to the boundary) at the centres of the

C boundary elements.

C complex PIPHI (MAXNEIG,MAXNPI): The velocity potential ( $\backslash$ phi $\}$ ) at

$\mathrm{C}$ the interior points.

C Working space

C complex WKSPC1 (MAXNSE, MAXNSE)

C complex WKSPC2 (MAXNSE, MAXNSE)

C complex WKSPC3 (MAXNSE, MAXNSE)

C logical WKSPC4(MAXNSE)

C complex WKSPO0 ((MAXNK-1)*MAXNSE, (MAXNK-1)*MAXNSE)

C complex WKSP01((MAXNK-1)*MAXNSE, (MAXNK-1)*MAXNSE)

C complex WKSP02((MAXNK-1)*MAXNSE, (MAXNK-1)*MAXNSE)

$C$ real WKSP03((MAXNK-1)*MAXNSE, (MAXNK-1)*MAXNSE)

C real WKSP04((MAXNK-1)*MAXNSE, (MAXNK-1)*MAXNSE)

C real WKSP05((MAXNK-1)*MAXNSE, (MAXNK-1)*MAXNSE) 
C real WKSP06((MAXNK-1)*MAXNSE, (MAXNK-1)*MAXNSE)

C real WKSP07((MAXNK-1)*MAXNSE)

$C$ real WKSP08((MAXNK-1)*MAXNSE)

C real WKSP09(MAXNK)

C integer WKSP10((MAXNK-1)*MAXNSE)

C complex WKSP11((MAXNK-1)*MAXNSE, (MAXNK-1)*MAXNSE)

C complex WKSP12((MAXNK-1)*MAXNSE)

The subroutine parameters that specify the interior two-dimensional Helmholtz problem must be set up in the main program. Let this be called MAIN.FOR. The following files must be linked together to construct the complete program:

$M A I N . F O R$ (and files containing any user-defined sub-programs),

AIBEM2.FOR,

H2LC.FOR, the file for computing the discrete operators - see Chapter 3,

FNHANK.FOR, the Hankel function - see Appendix 5,

INTEIG.FOR, the file for computing the solution to a linear system - see Appendix 4, GEOM2D.FOR, the file for 2D geometry - see Appendix 6.

\subsubsection{Subroutine MBEM3}

Subroutine MBEM3 computes the modal solutions of the three-dimensional Helmholtz equation in the domain interior to a closed surface and in a predetermined frequency range. The boundary $(S)$ is approximated by a set of planar triangular elements.

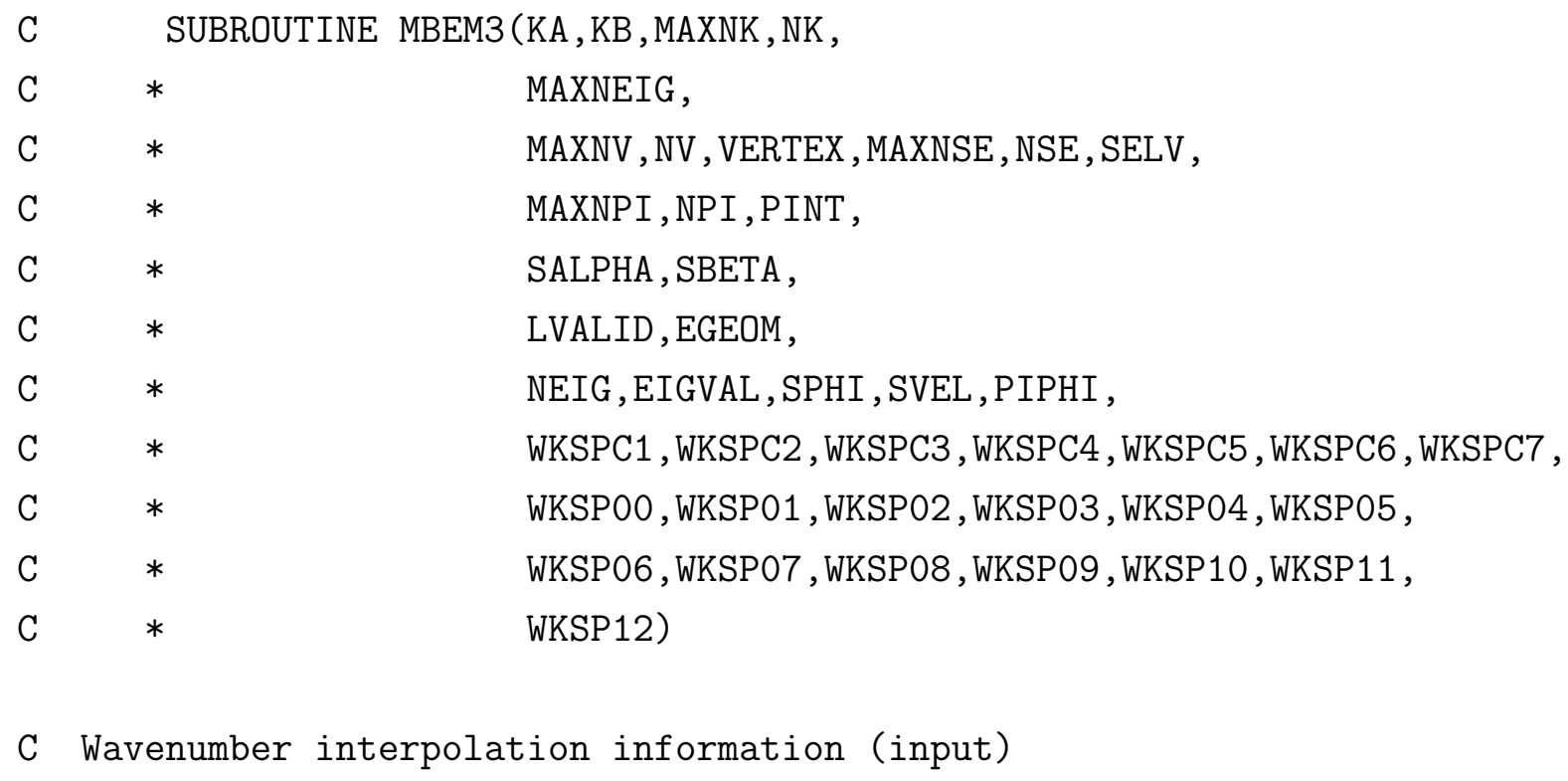




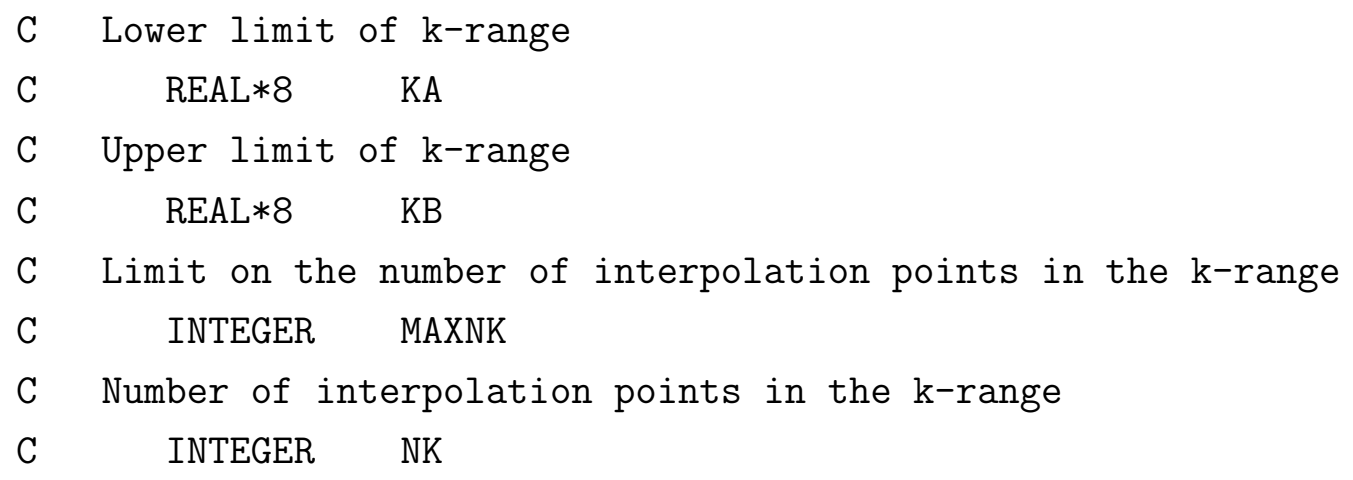

C Geometry of the boundary S (input)

$C$ integer MAXNV: The limit on the number of vertices of the polygon

C that defines (approximates) S. MAXNV $>=3$.

C integer NV: The number of vertices on $\mathrm{S} .3<=\mathrm{NV}<=\mathrm{MAXNV}$.

C real VERTEX (MAXNV,3): The coordinates of the vertices. VERTEX $(i, 1)$,

C $\operatorname{VERTEX}(i, 2), \operatorname{VERTEX}(i, 3)$ are the $x, y, z$ coordinates of the $i$-th vertex.

$C$ integer MAXNSE: The limit on the number of elements describing $S$.

C MAXNSE $>=3$.

C integer NSE: The number of elements describing S. $3<=N S E<=$ MAXNSE.

$C$ integer SELV(MAXNSE,3): The indices of the three vertices defining

$C$ each element. The $i$-th element have vertices

C $(\operatorname{VerteX}(\operatorname{SeLV}(i, 1), 1), \operatorname{VerteX}(\operatorname{SeLV}(i, 1), 2), \operatorname{VerTEX}(\operatorname{SeLV}(i, 1), 3))$,

C $(\operatorname{VerteX}(\operatorname{SeLV}(i, 2), 1), \operatorname{VerteX}(\operatorname{SeLV}(i, 2), 2), \operatorname{VERTEX}(\operatorname{SeLV}(i, 2), 3))$ and

C $(\operatorname{VerteX}(\operatorname{SeLV}(i, 3), 1), \operatorname{VerTeX}(\operatorname{SeLV}(i, 3), 2), \operatorname{VerTEX}(\operatorname{SeLV}(i, 3), 3))$.

C Interior points at which the solution is to be observed (input)

$C$ integer MAXNPI: Limit on the number of points interior to the

C boundary. MAXNPI $>=1$.

$C$ integer NPI: The number of interior points. $0<=$ NPI $<=$ MAXNPI.

$C$ real PINT(MAXNPI,3). The coordinates of the interior point.

C PINT( $i, 1), \operatorname{PINT}(i, 2), \operatorname{PINT}(i, 3)$ are the $x, y$ coordinates of the $i$-th

C point.

C The boundary condition ( $\{\backslash$ alpha $\}\{\backslash$ phi $\}+\{\backslash$ beta $\} \mathrm{v}=0$ ) (input)

C complex SALPHA (MAXNSE): The values of $\{\backslash$ alpha\} at the centres

C of the elements.

C complex SBETA(MAXNSE): The values of $\{\backslash$ beta $\}$ at the centres

$\mathrm{C}$ of the elements. 
C Validation and control parameters (input)

C logical LVALID: A switch to enable the choice of checking of

C subroutine parameters.

C real EGEOM: The maximum absolute error in the parameters that

C describe the geometry.

C Solution (output)

$C$ integer NEIG: The number of eigenvalues.

C complex EIGVAL(MAXNEIG): The eigenvalues.

C complex SPHI(MAXNEIG, MAXNSE): The velocity potential ( $\{\backslash$ phi $\}$ ) at

$\mathrm{C}$ the centres of the boundary elements.

C complex SVEL(MAXNEIG, MAXNSE): The velocity (v or $\mathrm{d}\{\backslash \mathrm{phi}\} / \mathrm{dn}$

$\mathrm{C}$ where $\mathrm{n}$ is the outward normal to the boundary) at the centres of the

C boundary elements.

C complex PIPHI(MAXNEIG, MAXNPI): The velocity potential ( $\backslash$ phi $\}$ ) at

$\mathrm{C}$ the interior points.

C Working space

C complex WKSPC1 (MAXNSE, MAXNSE)

C complex WKSPC2(MAXNSE, MAXNSE)

C complex WKSPC3 (MAXNSE, MAXNSE)

C logical WKSPC4(MAXNSE)

C complex WKSPOO((MAXNK-1)*MAXNSE, (MAXNK-1)*MAXNSE)

C complex WKSP01((MAXNK-1)*MAXNSE, (MAXNK-1)*MAXNSE)

C complex WKSP02((MAXNK-1)*MAXNSE, (MAXNK-1)*MAXNSE)

C real WKSP03((MAXNK-1)*MAXNSE, (MAXNK-1)*MAXNSE)

C real WKSP04((MAXNK-1)*MAXNSE, (MAXNK-1)*MAXNSE)

$C$ real WKSP05((MAXNK-1)*MAXNSE, (MAXNK-1)*MAXNSE)

C real WKSP06((MAXNK-1)*MAXNSE, (MAXNK-1)*MAXNSE)

$C$ real WKSP07 ((MAXNK-1)*MAXNSE)

C real WKSP08((MAXNK-1)*MAXNSE)

C real WKSP09(MAXNK)

C integer WKSP10((MAXNK-1)*MAXNSE)

C complex WKSP11((MAXNK-1)*MAXNSE, (MAXNK-1)*MAXNSE)

C complex WKSP12((MAXNK-1)*MAXNSE) 
The subroutine parameters that specify the exterior three-dimensional Helmholtz problem must be set up in the main program. Let this be called MAIN.FOR. The following files must be linked together to construct the complete program:

$M A I N . F O R$ (and files containing any user-defined sub-programs),

AEBEM3.FOR,

H3LC.FOR, the file for computing the discrete operators - see Chapter 3,

INTEIG.FOR, the file for computing the solution to a linear system - see Appendix 4, GEOM3D.FOR, the file for 3D geometry - see Appendix 6.

\subsubsection{Subroutine MBEMA}

Subroutine MBEMA computes the modal solutions of the axisymmetric three-dimensional Helmholtz equation in the domain interior to a closed surface and in a predetermined frequency range. The boundary $(S)$ is approximated by a set of conical elements.

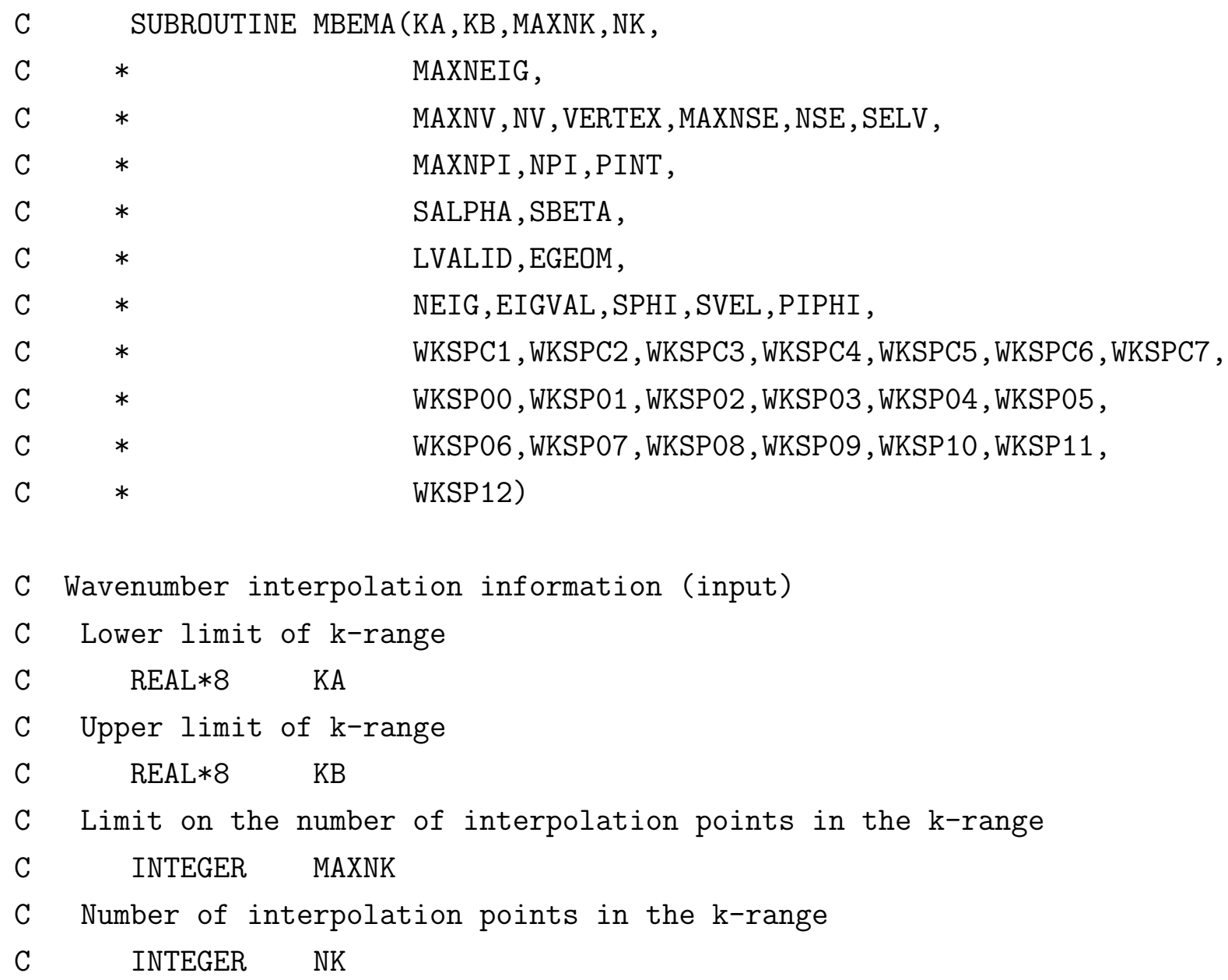


C Geometry of the boundary S (input)

$C$ integer MAXNV: The limit on the number of vertices of the polygon

C that defines (approximates) $S$. MAXNV $>=3$.

C integer NV: The number of vertices on $\mathrm{S} .3<=\mathrm{NV}<=\mathrm{MAXNV}$.

C real VERTEX (MAXNV,2): The coordinates of the vertices. VERTEX $(i, 1)$,

$C \operatorname{VERTEX}(i, 2)$ are the $\mathrm{x}, \mathrm{y}$ coordinates of the $i$-th vertex.

$C$ integer MAXNSE: The limit on the number of elements describing $S$.

C MAXNSE $>=3$.

$C$ integer NSE: The number of elements describing $\mathrm{S} .3<=\mathrm{NSE}<=$ MAXNSE.

$C$ integer SELV(MAXNSE,2): The indices of the two vertices defining

$\mathrm{C}$ each element. The i-th element have vertices

C $(\operatorname{Vertex}(\operatorname{SelV}(i, 1), 1), \operatorname{Vertex}(\operatorname{SelV}(i, 1), 2))$ and

C $(\operatorname{VerteX}(\operatorname{SELV}(i, 2), 1), \operatorname{VerteX}(\operatorname{SELV}(i, 2), 2))$.

C Interior points at which the solution is to be observed (input)

$C$ integer MAXNPI: Limit on the number of points interior to the

C boundary. MAXNPI $>=1$.

$C$ integer NPI: The number of interior points. $0<=N P I<=M A X N P I$.

$C$ real PINT(MAXNPI,2). The coordinates of the interior point.

C PINT $(i, 1), \operatorname{PINT}(i, 2)$ are the $x, y$ coordinates of the $i$-th point.

C The boundary condition ( $\{\backslash$ alpha $\}\{\backslash$ phi $\}+\{\backslash$ beta $\} \mathrm{v}=0$ ) (input)

C complex SALPHA(MAXNSE): The values of $\{\backslash$ alpha\} at the centres

C of the elements.

C complex SBETA(MAXNSE): The values of $\{\backslash$ beta $\}$ at the centres

C of the elements.

C Validation and control parameters (input)

C logical LVALID: A switch to enable the choice of checking of

C subroutine parameters.

C real EGEOM: The maximum absolute error in the parameters that

C describe the geometry.

C Solution (output)

$C$ integer NEIG: The number of eigenvalues.

C complex EIGVAL(MAXNEIG): The eigenvalues.

C complex SPHI(MAXNEIG, MAXNSE): The velocity potential ( $\backslash \backslash$ phi $\}$ ) at 


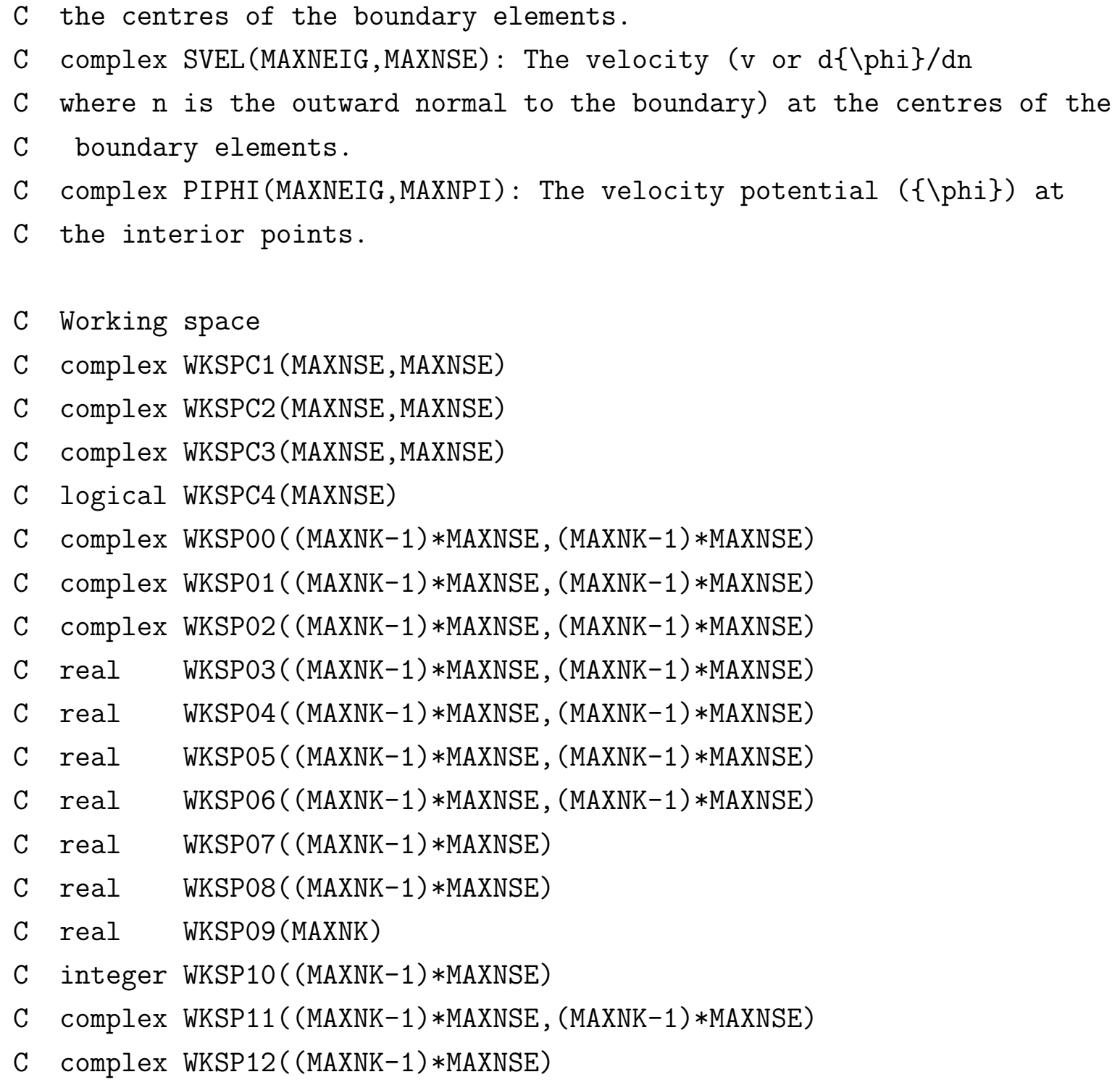

The subroutine parameters that specify the exterior three-dimensional axisymmetric Helmholtz problem must be set up in the main program. Let this be called MAIN.FOR. The following files must be linked together to construct the complete program:

MAIN.FOR (and files containing any user-defined sub-programs), AIBEMA.FOR, H3ALC.FOR, the file for computing the discrete operators - see Chapter 3, INTEIG.FOR, the file for computing the solution to a linear system - see Appendix 4, GEOM2D.FOR, the file for 2D geometry - see Appendix 6, GEOM3D.FOR, the file for 3D geometry - see Appendix 6 . 


\subsection{Test problems}

In this Section the subroutines MBEM2, MBEM3 and MBEMA are demontrated through invoking them from a main program and comparing the results with analytic solutions. The corresponding main programs are MBEM2_T, MBEM3_T and MBEMA_T.

\subsubsection{Program MBEM2_T}

The main program MBEM2_T tests module MBEM2, the subroutine for computing the resonant frequencies and mode shapes of an enclosed fluid in two dimensions. In MBEM2_T the domain is the interior of a square of side $0.1 \mathrm{~m}$ with the boundary represented by 32 elements. The representation of the boundary is described fully in Section 2.2 by the data structures VERTEX and SELV, see Tables 2.A and 2.B. Full results are given in file MBEM2.OUT.

The acoustic medium is air at 20 celcius and 1 atmosphere so that the speed of sound $c$ is $344 \mathrm{~m} / \mathrm{s}$. The program MBEM2_T contains three test problems. In the first test the mode(s) of the pure Neumann problem is sought in the frequency range $[40 \mathrm{~Hz}, 60 \mathrm{~Hz}]$ with $\mathrm{NK}=3$ (quadratic interpolation) whereas in the second test the same modes are sought in the range $[40 \mathrm{~Hz}, 50 \mathrm{~Hz}]$ with $\mathrm{NK}=4$ (cubic interpolation). The mode exists in both of these frequency ranges and satisfies both the Helmholtz equation and the homogeneous boundary condition is of the form $\varphi(\mathbf{p})=\sin \left(10 \pi p_{1}\right) \sin \left(10 \pi p_{2}\right)$ with the corresponding resonant wavenumber of $10 \sqrt{2} \pi$ which equals $44.4288(\mathrm{~Hz})$ to four decimal places. In the first test the resonant frequency is approximated by 44.2092 whereas in the second test problem the resonant frequency is approximated by 44.4804. In general the shorter the wavenumber range the more accurate the results are (up to a point) and this is confirmed by these examples.

In the third test problem the square is assigned the Dirichlet condition on two adjacent sides and a Neumann condition on the other two adjacent sides. Under these conditions the first mode is of the form $\varphi^{*}(\mathbf{p})=\sin \left(5 \pi p_{1}\right) \sin \left(5 \pi p_{2}\right)$ with corresponding resonant wavenumber $5 \sqrt{2} \pi$ which equals 22.2144 to four decimal places. In the test the solution is sought in the wavenumber range $[20 \mathrm{~Hz}, 30 \mathrm{~Hz}]$ and the approximation to the wavenumber is 22.2316 . The exact and computed values of the mode shape at five points in the interior are listed in Table 6.A. Note that the modal analysis programs assign the value $\varphi=1$ to the maximum value of $\varphi$ at the selected interior points hence in this case the exact solution is $\varphi^{*}(\mathbf{p})=\sin \left(5 \pi p_{1}\right) \sin \left(5 \pi p_{2}\right) / 0.83553$. 


\begin{tabular}{|c||c|c|}
\hline \multicolumn{2}{|c|}{ Table 6.A: Results from MBEM2_T } \\
\hline point & exact solution & numerical solution \\
\hline$(0.025,0.025)$ & 0.1716 & $0.1729+i 0.0028$ \\
$(0.025,0.075)$ & 0.4142 & $0.4138+i 0.0017$ \\
$(0.075,0.025)$ & 0.4142 & $0.4138+i 0.0017$ \\
$(0.075,0.075)$ & 1.0000 & $1.0000+i 0.0000$ \\
$(0.05,0.05)$ & 0.5858 & $0.5862+i 0.0018$ \\
\hline
\end{tabular}

\subsubsection{Program MBEM3_T}

The main program MBEM3_T tests module MBEM3, the subroutine for computing the resonant frequencies and mode shapes of an enclosed fluid in three dimensions. In MBEM3_T the domain is the interior of a sphere of side $1 \mathrm{~m}$ with the boundary represented by 36 elements. The representation of the boundary is described fully in Section 2.3 by the data structures VERTEX and SELV, see Tables 2.C and 2.D. Full results are given in file MBEM3.OUT.

The program MBEM3_T contains two test problems. In the first test the mode(s) of the pure Dirichlet problem is sought in the wavenumber range $[3,4]$ with $\mathrm{NK}=4$. The approximation to the characteristic wavenumber $\pi$ is 3.5567. This is equivalent to 194.7 $\mathrm{Hz}$ in the acoustic medium of air.

In the second test the Neumann mode is sought in the range [2,3]. The program returns three results 2.4068, 2.4071 and 2.3163. However, these are all approximations to the same characteristic wavenumber of 2.0816. The approximation occurs three times because this is a repeated eigenvalue.

The results show that the approximate wavenumber is significantly greater than the exact wavenumber in both cases. This can be explained simply by observing that the effect of the boundary approximation is to significantly lessen the size of the sphere and the larger eigenvalues reflect this.

\subsubsection{Program MBEMA_T}

The main program MBEMA_T tests module MBEMA, the subroutine for computing the resonant frequencies and mode shapes of an enclosed fluid in three dimensions. In MBEMA_T the domain is the interior of a sphere of side $1 \mathrm{~m}$ with the boundary represented by 16 truncated conical elements. The representation of the boundary is described fully in Section 2.4 by the data structures VERTEX and SELV, see Tables 
2.E and 2.F. Full results are given in file MBEMA.OUT.

In the first test problem the Dirichlet modes are sought in the range $[3,5]$ with $\mathrm{NK}=2$ (linear interpolation). The approximations 3.138 and 4.512 are found to the exact solutions of 3.142 and 4.493. In the acoustic medium of water the approximate eigenfrequencies are $761 \mathrm{~Hz}$ and $1094 \mathrm{~Hz}$.

In the second test problem the Neumann modes are sought in the range $[2,4]$ with $\mathrm{NK}=4$ (cubic interpolation). The approximations 2.089 and 3.360 are found to the exact solutions of 2.082 and 3.342 .

\subsection{Application: Loudspeaker Enclosure}

In this Section a modal analysis of the air-tight interior of a test axially symmetric loudspeaker is carried out via the boundary element method using subroutine MBEMA. Some of the results are compared with results from physical experiment. For further details on this application and the results obtained the reader is referred to [48].

\subsubsection{Background}

The effect of the fluid-loading of the air on the cone is of some interest to loudspeaker designers. The coupling of air external to the loudspeaker to the motion of the cone is considered experimentally in Jones [35], wherein the difference between the forced vibration of a cone in air and its vibration in a vacuum is found to be negligible. However, it is expected that the presence of the air inside the cabinet can have a significant effect on the vibration of the cone due to the relatively small, enclosed volume occupied by the air.

The greatest effect of air loading on the vibration of the cone occurs when there are large changes in pressure over the surface of the cone. The maximum change in pressure will be at acoustic resonant frequencies and therefore the corresponding mode shapes are studied. By applying the methods to an axisymmetric loudspeaker, the acoustic properties may be examined whilst reducing the dimension of the problem by one and thus reducing the computational expense when compared to the full three-dimensional analysis that is necessary for a general loudspeaker design. In addition, considerable previous work has been done on the structural vibration of axially symmetric loudspeaker drive units (see Jones [35] and Jones and Henwood 
[36], for example). In this work we show results for the cabinet shown in Figure 6.1, which is basically a cylinder $120 \mathrm{~mm}$ deep and $132 \mathrm{~mm}$ in diameter. The loudspeaker has a conical drive unit of radius $80 \mathrm{~mm}$ fitted. Given these dimensions, the lowest cabinet resonance occurs around $1 \mathrm{kHz}$, and the cabinet resonances can be observed in sound pressure measurements outside the cabinet.

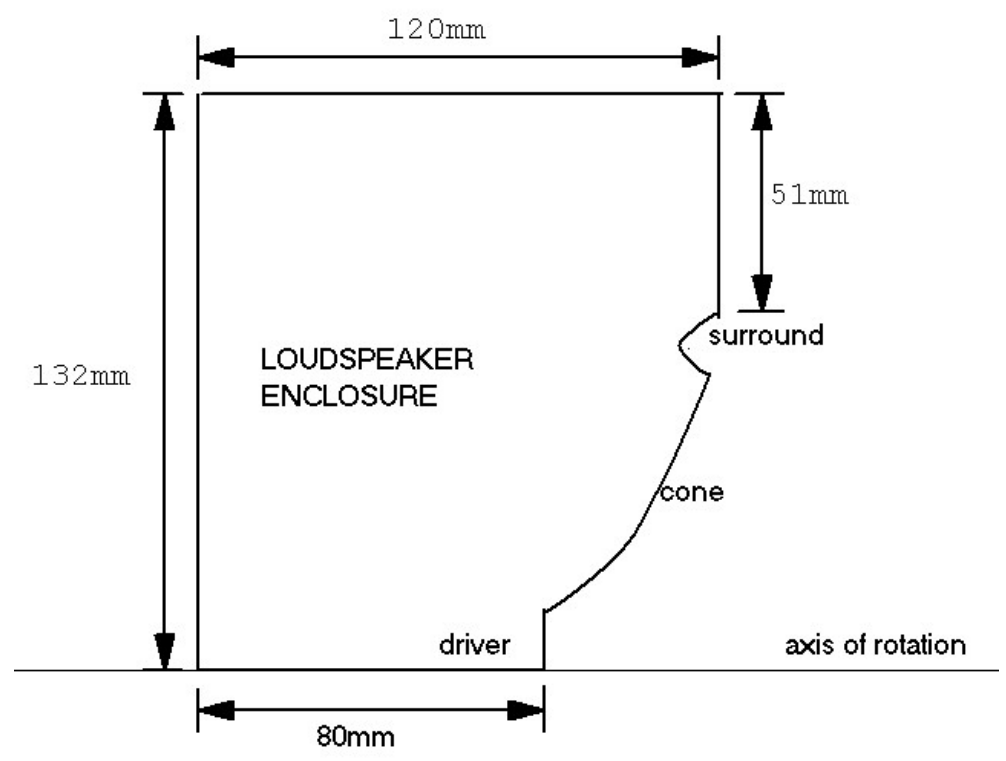

Fig. 6.1. Diagram of the axisymmetric cabinet. 


\subsubsection{Particular implementations of computational method and Measurements}

For the application of the boundary element method the boundary of the generator of the loudspeaker is approximated by 32 conical elements of approximately equal length along the generator, as shown in Figure 6.1. Solutions were sought in the $k$ ranges $[0.0,5.0],[5.0,10.0],[10.0,15.0]$ and so on. In each range quadratic interpolation was applied $(\mathrm{NK}=3)$. The approximations to the mode shapes are then obtained at around 100 points in the interior.

For the measurement of the resonant frequencies, microphone readings of the sound pressure were taken at various positions within the cabinet. Measurements were made through all frequencies of interest, commencing at $20 \mathrm{~Hz}$ and going through to $20 \mathrm{kHz}$. The peaks in the response inform us of the internal resonant frequencies.

\subsubsection{Results}

Firstly the five lowest resonant frequencies obtained through the boundary element methods and the results obtained by measurement are compared in Table 6.B.

\begin{tabular}{|c|c|c|}
\hline \multicolumn{3}{|c|}{ Table 6.B: Computed and measured loudspeaker resonant frequencies } \\
\hline Mode & Boundary Element & Experimental \\
\hline 1 & $1414 \mathrm{~Hz}$ & $1318 \mathrm{~Hz}$ \\
2 & $1590 \mathrm{~Hz}$ & $1679 \mathrm{~Hz}$ \\
3 & $2232 \mathrm{~Hz}$ & $2133 \mathrm{~Hz}$ \\
4 & $2815 \mathrm{~Hz}$ & $2691 \mathrm{~Hz}$ \\
5 & $2876 \mathrm{~Hz}$ & $3306 \mathrm{~Hz}$ \\
\hline
\end{tabular}

The major contribution to the discrepancy between the measured and calculated values is believed to be the simplicity of the model chosen, which fails to include any internal structure to the loudspeaker. In addition, the maximum pressure occurs at slightly different frequencies for different microphone positions.

Figure 6.2 shows the third and fifth mode shapes obtained via the boundary element method. The mode shapes are constructed from the returned values of $\varphi^{*}$ in the domain. The values on the contours are arbitrary. 


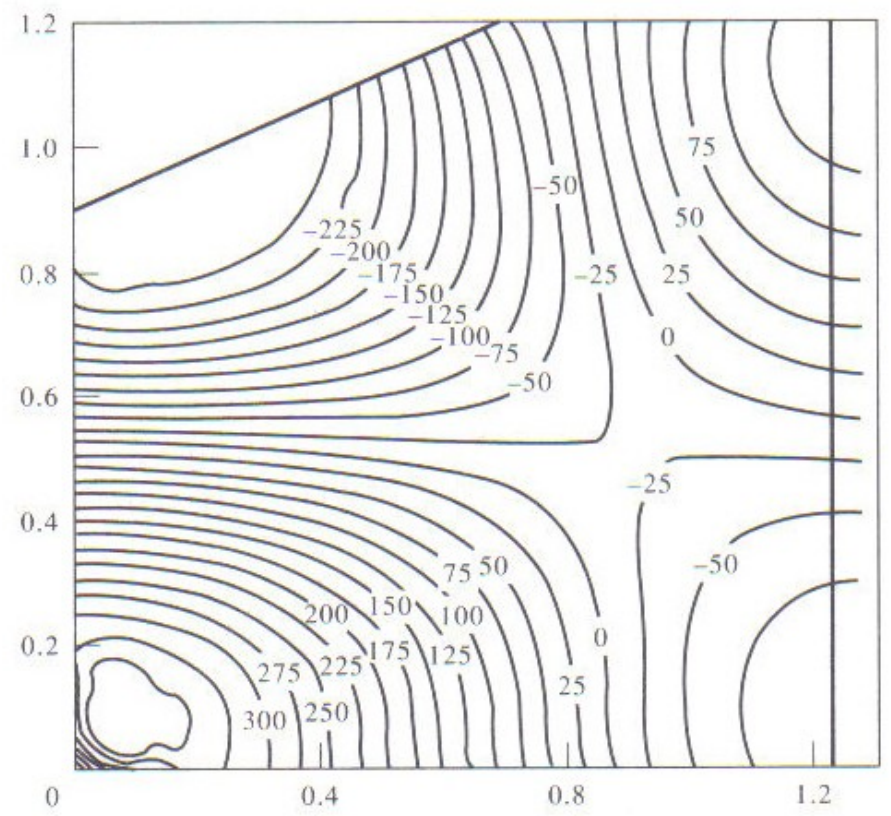

Fig 6.2(a). The third mode shape of the loudspeaker cabinet.

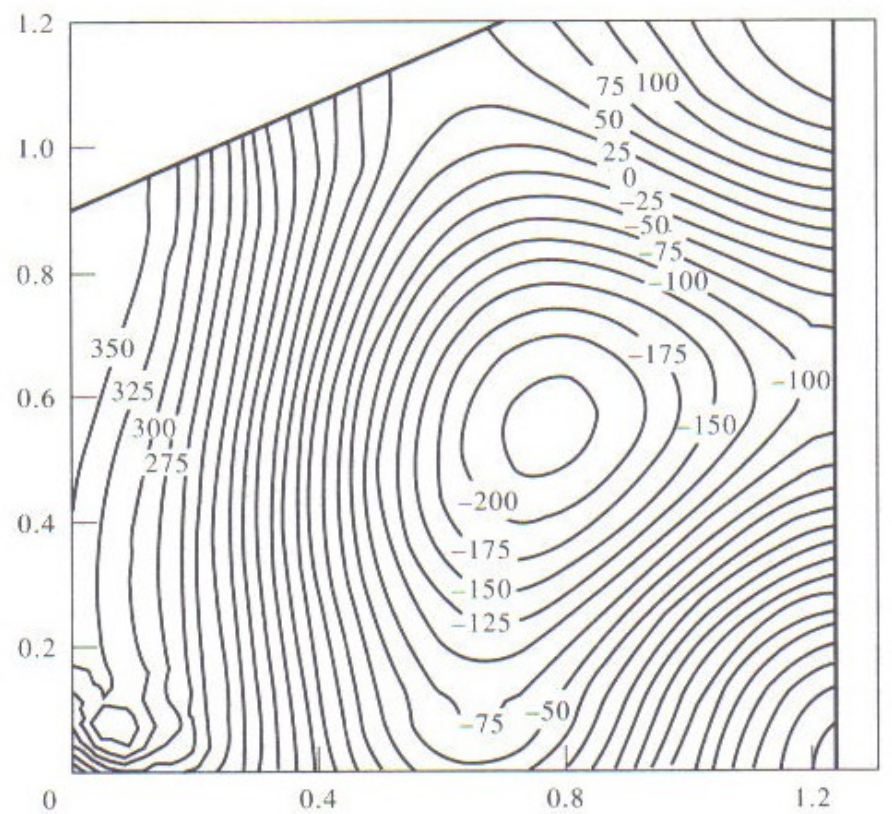

Fig 6.2(b). The fifth mode shape of the loudspeaker cabinet. 


\subsection{Conclusion}

It may seem unnecessary to develop the boundary element method for the solution of interior acoustic modal analysis or the interior boundary value problem since such problems can satisfactorily solved by the more traditional finite element method. Furthermore, no claim is being made that the BEM is generally more efficient than the finite element method in these applications, although in some cases it is easier to apply since only the boundary requires discretisation. However, in software libraries for solving acoustic problems based on the boundary element method, such as the one that accompanies this text, it would be anomalous not to provide a method for solving the modal analysis poblem. Besides, the underlying technique has been applied to the modal analysis of structures in contact with fluids in Kirkup and Amini [41] such problems can be difficult to solve by other methods.

Underlying the solution of the modal analysis problem by the BEM is a method for solving a non-linear eigenvalue problem. The method that is derived through frequency interpolation of the matrix described in Section 6.3 is a flexible and consistent method for the modal analysis of the interior acoustic problem. However, it may be possible to develop more efficient methods than the QZ algorithm for solving the eigenvalue problem (6.12).

The subroutines described in this Section are flexible in that the wavenumber range over which the resonant frequencies are sought and the number of interpolation points within that range are parameters. In general the sequence of resonant frequencies can be obtained by stepping through the full wavenumber range covering a fixed subinterval at each stage. Ideally users need to determine the wavenumber interval and the number of interpolation points used in each interval to obtain satisfactory solutions with minimum processing time. It is beneficial for users to gain some experience with the methods before using them in practical situations.

The results from the test problems show that the boundary element method can be confidently applied to the modal analysis problem. The comparison of numerical and experimental results in the application of the methods to the loudspeaker enclosure show that the BEM is able to extract the resonant frequencies and mode shapes of an enclosure in a practical application. The mode shapes shown in the figures are comparable with those obtained by the finite element method, see Kirkup and Jones [48]. 


\section{Bibliography}

[1] J. O-O. Adeyeye, M. J. M. Bernal and K. E. Pitman (1985). An Improved Boundary Integral Equation Method for Helmholtz Equations, International Journal for Numerical Methods in Engineering, 21, 779-787.

[2] A. Ali, C. Rajakumar and S. M. Yunus (1991). On the Formulation of the Acoustic Boundary Element Eigenvalue Problems, International Journal of Numerical Methods in Engineering, 31, 1271-1282.

[3] S. Amini, P. J. Harris and D. T. Wilton (1992). Coupled Boundary and Finite Element Methods for the Solution of the Dynamic Fluid-Structure Interaction Problem, in Lecture Notes in Engineering 77, Edited by C. A. Brebbia and S. A. Orszag, Springer-Verlag.

[4] S. Amini and S. M. Kirkup (1995). Solution of Helmholtz Equation in the Exterior Domain by Elementary Boundary Integral Methods, Journal of Computational Physics, 118, 208-221.

[5] C. T. H. Baker (1977). The Numerical Treatment of Integral Equations, Clarendon Press, Oxford.

[6] R. P. Banaugh and W. Goldsmith (1963). Diffraction of Steady Acoustic Waves by Surfaces of Arbitrary Shape, Journal of the Acoustical Society of America, bf 35(10), 1590-1601.

[7] P. K. Banerjee and R. Butterfield (1981). Boundary Element Methods in Engineering Science, McGraw-Hill.

[8] P. K. Banerjee, S. Ahmad and H. C. Wang (1988). A New BEM Formulation for the Acoustic Eigenfrequency Analysis, International Journal for Numerical Methods in Engineering, 26, 1299-1309. 
[9] W. A. Bell, W. L. Meyer and B.T. Zinn (1977). Predicting the acoustics of arbitrarily shaped bodies using an integral approach, AIAA Journal, 15(6), 813820.

[10] J. Ben Mariem and M. A. Hamdi (1987). A new Boundary Element Method for Fluid-Structure Interaction Problems, International Journal of Numerical Methods in Engineering, 24, 1251-1267.

[11] R. J. Bernhard, B. K. Gardner, C. G. Mollo and C. R. Kipp (1987). Prediction of Sound Fields in Cavities Using Boundary-Element Methods, AIAA Journal, 25, 1176-1183.

[12] H. Brakhage and P. Werner (1965). Über das Dirichletsche Außenraumproblem für die Helmholtzsche Schwingungsgleichung, Archiv. der Math, 16, 325-329.

[13] C. A. Brebbia (1978). The Boundary Element Method for Engineers, Pentech Press.

[14] G. B. Brundrit (1964). A Solution to the Problem of Scalar Scattering from a Smooth, Bounded Obstacle using Integral Equations. Quarterly Journal of Mechechanics and Applied Mathematics, Vol XVIII, Pt. 4, 473-489.

[15] A. J. Burton and G. F. Miller (1971). The Application of Integral Equation Methods to the Numerical Solution of some Exterior Boundary Value Problems, Proc. Royal Society, London, A323, 201-210.

[16] A. J. Burton (1973). The Solution of Helmholtz Equation in Exterior Domains using Integral Equations. NPL Report NAC30, National Physical Laboratory, Teddington, Middlesex, UK.

[17] A. J. Burton (1976). Numerical Solution of Acoustic Radiation Problems, NPL Report OC5/535, National Physical Laboratory, Teddington, Middlesex, UK.

[18] L. H. Chen and D. G. Schweikert (1963). Sound Radiation from an Arbitrary Body, Journal of the Acoustical Society of America, 35(10), 1626-1632.

[19] C. Y. R. Cheng, A. F. Seybert and T. W. Wu (1991). A Multidomain Boundary Element Solution for Silencer and Muffler Performance Prediction. Journal of Sound and Vibration, 151(1), 119-129.

[20] G. Chen and J. Zhou (1992). Boundary Element Methods, Academic Press. 
www.boundary-element-method.com

[21] G. Chertock (1964). Sound Radiation from Vibrating Bodies, Journal of the Acoustical Society of America, 36(7), 1305-1313.

[22] R. D. Ciskowski and C. A. Brebbia (1991). Boundary Element Methods in Acoustics. Computational Mechanics Publications, Southampton.

[23] D. Colton and R. Kress (1983). Integral Equation Methods in Scattering Theory, John Wiley and Sons, New York.

[24] J. P. Coyette and K. R. Fyfe (1990). An Improved Formulation for Acoustic Eigenmode Extraction from Boundary Element Models, ASME Journal of Vibration and Acoustics, 112, 392-398.

[25] P. A. Davis and P. Rabinowitz (1984). Methods of Numerical Integration, Academic Press, Oxford.

[26] L. M. Delves and J. L. Mohamed (1985) Computational Methods for Integral Equations, Cambridge University Press.

[27] G. De Mey (1976). Calculation of Eigenvalues of the Helmholtz Equation by an Integral Equation, International Journal for Numerical Methods in Engineering, 10, 59-66.

[28] I. Gohberg, P. Lancaster and L. Rodman (1982). Matrix Polynomials, Academic Press.

[29] W. S. Hall (1994) The Boundary Element Method, Kluwer Academic Publishers Group, The Netherlands.

[30] I. Harari and T. J. R. Hughes (1991). Finite Element Methods for the Helmholtz Equation in an Exterior Domain: Model Problems, Computer Methods in Applied Mechanics and Engineering, 87, 59-96.

[31] Jeong-Guon Ih, Bong-Ki Kim and Won-Sik Choo (1995). Comparison of Eigenvalue Analysis Techniques in Acoustic Boundary Element Method, Euro-Noise 95, 591-596.

[32] MATH/LIBRARY - Fortran routines for mathematical applications (1987). MALB-USM-PERFECT-1.0, IMSL, Houston.

[33] www.boundary-element-method.com

[34] M. A. Jaswon and G. T. Symm (1977). Integral Equation Methods in Potential Theory and Elastostatics, Academic Press. 
[35] C. J. C. Jones (1986). Finite Element Analysis of Loudspeaker Diaphragm Vibration and Prediction of the Resulting Sound Radiation, PhD thesis, Brighton Polytechnic, Brighton, UK.

[36] M. A. Jones, L. A. Binks and D. J. Henwood (1991). Finite Element Methods Applied to the Analysis of High Fidelity Loudspeaker Transducers, Computers and Structures 44(4), 765-772.

[37] P. Juhl (1994). A Numerical Study of the Coefficient Matrix of the Boundary Element Method Near Characteristic Frequencies, Journal of Sound and Vibration, 175(1), 39-50.

[38] P. Juhl (1998). A note on the Convergence of the Direct Collocation Boundary Element Method, Journal of Sound and Vibration, 212(4), 703-719.

[39] C. R. Kipp and R. J. Bernhard (1987). Prediction of Acoustical Behavior in Cavities using an Indirect Boundary Element Method, ASME Journal of Vibration and Acoustics, 109, 22-28.

[40] S. M. Kirkup (1989). Solution of Exterior Acoustic Problems by the Boundary Element Method, PhD thesis, Brighton Polytechnic, Brighton, UK.

[41] S. M. Kirkup and S. Amini (1991). Modal Analysis of Acoustically-loaded Structures via Integral Equation Methods, Computers and Structures, 40(5), 12791285 .

[42] S. M. Kirkup (1991). The Computational Modelling of Acoustic Shields by the Boundary and Shell Element Method, Computers and Structures, 40(5), 1177 1183.

[43] S. M. Kirkup and D. J. Henwood (1992). Computational Solution of Acoustic Radiation Problems by Kussmaul's Boundary Element Method, Journal of Sound and Vibration, 152(2), 388-402.

[44] S. M. Kirkup and D. J. Henwood (1992). Methods for speeding up the Boundary Element Solution of Acoustic Radiation Problems, Trans. ASME Journal of Vibration and Acoustics, 114(3), 374-380.

[45] S. M. Kirkup and R. J. Tyrrell (1992). Computer-Aided Analysis of Engine Noise, International Journal of Vehicle Design, 13(4), 388-402. 
[46] S. M. Kirkup and S. Amini (1993). Solution of the Helmholtz Eigenvalue Problem via the Boundary Element Method, International Journal for Numerical Methods in Engineering 36(2), 321-330.

[47] S. M. Kirkup (1994). The Boundary and Shell Element Method, Applied Mathematical Modelling, 18, 418-422, (1994).

[48] S. M. Kirkup and M. A. Jones (1996). Computational Methods for the Acoustic Modal Analysis of an Enclosed Fluid with application to a Loudspeaker Cabinet, Applied Acoustics, 48(4), 275-299.

[49] S. M. Kirkup (1997). Solution of Helmholtz Problems in Discontinuous Domains via the Boundary and Shell Element Method, Computer Methods in Applied Mechanics and Engineering, 140 3-4, 393-404.

[50] S. M. Kirkup (1998). Fortran Codes for Computing the Discrete Helmholtz Integral Operators, to appear in Advances in Computational Mathematics.

[51] R. E. Kleinmann and G. F. Roach (1974). Boundary Integral Equations for the three-dimensional Helmholtz Equation SIAM Review, 16 (2), 214-236.

[52] G. H. Koopman and H. Benner (1982). Method for Computing the Sound Power of Machines based on the Helmholtz Integral, The Journal of the Acoustical Society of America, 71(1), 78-89. Oxford University Press, Oxford.

[53] S. Kopuz and N. Lalor (1995). Analysis of Interior Acoustic Fields Using the Finite Element Method and the Boundary Element Method, Applied Acoustics, 45, 193-210.

[54] R. Kussmaul (1969). Ein numeriches Verfahren zur Lösung des Neumannschen $\mathrm{Au} \beta$ enraumproblems für die Helmholtsche Schwingungsgleichung. Computing 4, 246-273.

[55] P. Lancaster (1977). A Review of Numerical Methods for Eigenvalue Problems Nonlinear in Parameter, Numerik und Andwendungen von Eigenwertaufgaben und Verzweigungsproblemen (edited by E. Bohl, L. Collatz and K. P. Hedeler) ISNM 38, Basel-Stuttgart, Birkhauser.

[56] M. E. Laursen and M. Gellert (1978). Some Criteria for Numerically Integrated Matrices and Quadrature Formulas for Triangles, International Journal for $\mathrm{Nu}$ merical Methods in Fluids, 12, 67-76. 
[57] R. Leis (1965). Zur Dirichletschen Randwertaufgabe des Außenraums der Schwingungsgleichung, Math. Z., 90, 205-211.

[58] Ya Yan Lu and Shing-Tung Yau (1991). Eigenvalues of the Laplacian through Boundary Integral Equations, SIAM J. Matrix Anal. Appl., 12(3), 597-609.

[59] I. C. Mathews (1986). Numerical Techniques for three-dimensional steady state Fluid-Structure Interaction, Journal of the Acoustical Society of America, 79(5), 1317-1325.

[60] W. L. Meyer, W. A. Bell, B. T. Zinn and M. P. Stallybrass (1978). Boundary Integral Solutions of three dimensional Acoustic Radiation Problems, Journal of Sound and Vibration, 59(2), 245-262.

[61] C. B. Moler and G. W. Stewart (1973). An Algorithm for Generalized Matrix Eigenvalue Problems, SIAM Journal of Numerical Analysis, 10(2), 241-256.

[62] P. M. Morse and K. U. Ingard (1981), Theoretical Acoustics, McGraw-Hill.

[63] NAG Fortran Library, The Numerical Algorithms Group Ltd, Oxford, UK.

[64] D. J. Nefske, J. A. Wolf and L. J. Howell (1982). Structural-Acoustic Finite Element Analysis of the Automobile Passenger Compartment: A Review of Current Practice, Journal of Sound and Vibration, 80, 247-266.

[65] D. J. Nefske and S. H. Sung (1985). Vehicle Interior Acoustic Design using Finite Element Methods, International Journal of Vehicle Design, 6(1), 24-39.

[66] O. I. Panich (1965). On the Question of the Solvability of the Exterior Boundary Problem for the Wave Equation and Maxwell's Equation, Uspeki Mat. Nauk, 20, $221-226$.

[67] G. Peters and J. H. Wilkinson (1970). $A x=\lambda B x$ and the Generalized Eigenproblem, SIAM Journal of Numerical Analysis, 7(4), 479-492.

[68] M. Petyt, J. Lea and G. H. Koopman (1976). A Finite Element Method for Determining the Acoustic Modes of Irregular Shaped Cavities, Journal of Sound and Vibration, 45(4), 495-502.

[69] M. Petyt (1983). Finite Element Techniques for Acoustics, in Theoretical Acoustics and Numerical Techniques edited by P. J. T Filippi, Springer-Verlag.

[70] W. H. Press, S. A. Teukolsky, W. T. Vetterling and B. P. Flannery (1992), Numerical Recipes in Fortran, Cambridge University Press. 
www.boundary-element-method.com

[71] A. D. Pierce (1981). Acoustics: An Introduction to its Physical Principles and Applications, McGraw-Hill.

[72] J. J. do Rego Silva (1994). Acoustic and Elastic Wave Scattering using Boundary Elements, Topics in Engineering 18, edited by C. A. Brebbia and J. J. Connor, Computational Mechanics Publications, Southampton and Boston.

[73] A. Ruhe (1973). Algorithms for the Non-Linear Eigenvalue Problem, SIAM Journal of Numerical Analysis, 10, 674-689.

[74] M. N. Sayhi, Y. Ousset and G. Verchery (1981). Solution of Radiation Problems by Collocation of Integral Formulations in terms of Single and Double Layer Potentials, Journal of Sound and Vibration, 74(2), 187-204.

[75] H. A. Schenck (1968). Improved Integral Formulation for Acoustic Radiation Problems, Journal of the Acoustical Society of America, 44(1), 41-58.

[76] A. F. Seybert and J. A. Holt (1985). A Technique for the Prediction of the Noise Field from an Arbitrary Vibrating Machine, Noise Con., 51-56.

[77] A. F. Seybert and T. K. Rengarajan (1987). The use of CHIEF to obtain unique solutions for acoustic radiation using boundary integral equations, Journal of the Acoustical Society of America, 81(5), 1299-1306.

[78] A. F. Seybert and T. K. Rengarajan (1987). Application of the Boundary Element Method to Acoustic Cavity Response and Muffler Analysis, ASME Journal of Vibration, Acoustics, Stress, and Reliability in Design, 119, 15-21.

[79] A. H. Stroud and D. Secrest (1966). Gaussian Quadrature Formulas, Prentice Hall.

[80] T. Terai (1980). On the Calculation of Sound Fields around three-dimensional Objects by Integral Equation Methods, Journal of Sound and Vibration, 69(1), 71-100.

[81] G. R. C. Tai and R. P. Shaw (1974). Helmholtz-equation Eigenvalues and Eigenmodes for Arbitrary Domains, Journal of the Acoustical Society of America, 56(3), 796-804.

[82] A. G. P. Warham (1989). The Helmholtz Integral Equation for a Thin Shell, NPL Report DITC 129/88, National Physical Laboratory, Teddington, Middlesex. 
[83] D. T. Wilton (1978). Acoustic Radiation and Scattering from Elastic Structures, International Journal for Numerical Methods in Engineering, 13, 123-128.

[84] R. Wobst (1987). The Generalized Eigenvalue Problem and Acoustic Surface Wave Computations, Computing, 39, 57-69. 


\section{Addendum}

The references given here are an addendum to the 1998 version of the book. These references give further work on the Boundary Element Method, especially in relation to the field of acoustics. A web reference is given where appropriate. The web page http://www.boundary-element-method.com/extra will be maintained to hold this growing list of references.

The author's papers are generally listed on http://www.kirkup.info/papers

M. Ben Abdillah, O. Pascal, H. Aubert (2004). Cross-shaped fractal antenna: A compact circularly polarized radiating element, Microwave and Optical Technology Letters 43(6) , $518-521$

A. Ali and C. Rajakumar (2004). The Boundary Element Method: Applications in Sound and Vibration, A. A. Balkema, The Netherlands.

M.H. Aliabadi (2002) The Boundary Element Method: Vol. 2: Applications in Solids and Structures, John Wiley and Sons Ltd.

S. Amini and N. D. Maines (1998). Preconditioned Krylov subspace methods for boundary element solution of the Helmholtz equation, 41(5), 875-898.

X. Antoine and M. Darbas (2005). Generalized Combined field Integral Equations for the iterative solution of the Helmholtz equation in three dimensions. http://www.acm.caltech.edu/ antoine/articles/GCFIE.pdf

X. Antoine and M. Darbas (2005). Alternative Integral Equations for the iterative solution of acoustic scattering problems, Q. Jl Mech. Appl. Math. 58 (1), 107128. http://qjmam.oxfordjournals.org/cgi/reprint/58/1/10\%.pdf

P. D'Antonio, and T. Cox (2004). Acoustic Absorbers and Diffusers: Theory, Design and Application, Spon Press.

B\&W. Development of the B\&W 800D and Nautilus 800 series louspeakers. 
J. Backman (2004). Image Source Model for Loudspeaker Enclosure. Audio Engineering Society, Preprint Number 6182, Convention 117.

http://www.aes.org/e-lib/browse.cfm?elib=12839

T. G. H. Basten (2001). Noise Reduction by Viscothermal Acousto-Elastic Interaction in Double Wall Panels, PhD-thesis, University of Twente, Enschede, The Netherlands. http://doc.utwente.nl/fid/1541

K. J. Bastyr and D. E. Capone (2003). On the Acoustic Radiation from a Loudspeaker's Cabinet, Journal of the Audio Engineering Society, 51(4), 234-243.

A. V. Boriskin, A. I. Nosich, S. V. Boriskina, P. Sewell, T. M. Benson and A. Altintas, (2004). Resonance lens antenna analysis for mm-wave applications, Physics and Engineering of Microwaves, Millimeter, and Submillimeter Waves, 632- 634 Vol.2

S. V. Boriskina, P. Sewell, T. M. Benson and A. I. Nosich (2004) Accurate simulation of two-dimensional optical microcavities with uniquely solvable boundary integral equations and trigonometric Galerkin discretization, J. Opt. Soc. Am. A, 21(3) 393-402.

http://www.geocities.com/sveta2004/Boriskina_JOSAA2004.pdf

D. C. Calvo (2004). A wide-angle on-surface radiation condition applied to scattering by spheroids, The Journal of the Acoustical Society of America, 116(3), 1549-1558.

J.T. Chen a, S.R. Lin, K.H. Chen, I.L. Chen and S.W. Chyuan (2003) Eigenanalysis for membranes with stringers using conventional BEM in conjunction with SVD technique, Comput. Methods Appl. Mech. Engrg. 192 1299-1322.

http://www.hre.ntou.edu.tw/msvlab/paper/cmame/cmame-03.pdf

Chen Ke (2005). Matrix Preconditioning Techniques and Applications, Cambridge University Press. 
R. Cunderlik , K. Mikula, AND M. Mojzes (2002) 3D BEM application to Neumann geodetic BVP using the collocation with linear basis functions, Proceedings of $A L$ GORITMY, Conference on Scientic Computing, 268-275. http://www.iam.fmph.uniba.sk/amuc/_contributed/algo2002/cunderlik/cunderlik.pdf

R. Cunderlik , K. Mikula, AND M. Mojzes (2004). A comparison of the variational solution to the Neumann geodetic boundary value problem with the geopotential model EGM-96, Contributions to Geophysics and Geodesy, 34(3), 209-225. http://www.math.sk/mikula/cmm.pdf

C. P. Davis, K. F. Warnick (2004). High Order Convergence with a Low Order Discretization of the 2D MFIE, Brigham Young University, Electrical And Computer Engineering. https://dspace.byu.edu/handle/1877/46

H. Djojodihardjo and E. Tendean (2004). Boundary Element Computational Technique for Structural Dynamics of Structure subjected to Acoustic Excitation, 55th International Astronautical Congress, Vancouver, Canada IAC-04-I.2.09.

M. Ehrhardt and A. Zisowsky (2005). Discrete nonlocal boundary conditions for SplitStep Pade Approximations of the One Way Helmholtz Equation, Journal of Computational and Applied Mathematics http://www.hyke.org/preprint/2005/09/096.pdf

D. O. Elorza (2005). Room acoustics modeling using the raytracing method: implementation and evaluation, Licentiate Thesis University of Turku Department of Physics.

http://www.ttl.fi/NR/rdonlyres/6E2C64C0-0F24-4050-B783-BC9B15401DBC/0/H8.pdf

T.J. Esward, K. Lees, D. Sayers, L. Wright (2004). Testing continuous modelling software: three case studies. NPL Report CMSC 42/04.

http://publications.npl.co.uk/npl_web/pdf/cmsc42.pdf 
T. Friese, F. Schmidt, David Yevick (1999). Transparent Boundary Conditions for a Wide-Angle Approximation of the One-Way Helmholtz Equation.

http://citeseer.ist.psu.edu/443134.html

E. Friot, R. Guillermin, and M. Winninger (2005). Active control of scattered acoustic radiation: a real-time implementation for a three-dimensional object. http://arxiv.org/PS_cache/physics/pdf/0511/0511164.pdf

P. Fryer, D. Henwood, J. Moore, G. Geaves, Verification of an approach for transient strucural simulation of loudspeakers incorporating damping, Audio Engineering Society Electronic Library http://www.aes.org/e-lib/browse.cfm?elib=9905

M. Furlan, A. Cernigoj, M. Boltezar (2003). A coupled electromagnetic-mechanicalacoustic model of a DC electric motor, COMPEL: The International Journal for Computation and Mathematics in Electrical and Electronic Engineering, 22(4), 11551165.

M. Furlan, M. Boltezar (2003). The Boundary Element Method in Acoustics - an Example of Evaluating the Sound Field of a DC Electric Motor. Journal of Mechanical Engineering, 50(2), 115-128.

http://www.fs.uni-lj.si/sv/English/2004/2/sv-02-an.pdf

M. Furlan, R.Rebec, A. Cernigoj, D. Celic, P. Cermelj and M. Boltezar (2006). Vibroacoustic modelling of an alternator, Journal of Mechanical Engineering, 52(2), 112125.

http://www.fs.uni-lj.si/sv/English/2004/2/sv-02-an.pdf

E. R. Geddes L. Lee (2002). Audio Transducers, Gedlee Associates.

R. Guror, B. Olsen, A. Kroese, F. Cook, S. Kumar (2004). Evaluation of critical components of non-ceramic insulators in-service: role of defective interfaces, Power Systems Engineering Research Center, Arizona State University http://www.pserc.org/cgi-pserc/getbig/publicatio/reports/2004report/psercreport.pdf 
U. Hohenester and J. Krenn (2005). Surface plasmon resonances of single and coupled metallic nanoparticles: A boundary integral method approach, Physical Review B 72, 195429.

http://nanooptics.uni-graz.at/ol/papers/PRB05_hohenester.pdf

D. S. Holloway, G. A. Thomas and M. R. Davis (2004). Boundary element methods in the prediction of the acoustic damping of ship whipping vibrations. ANZIAM J. 45 (E) C845C856.

http://anziamj.austms.org.au/V45/CTAC2003/Hol2/Hol2.pdf

P. Juhl (1998). A note on the Convergence of the Direct Collocation Boundary Element Method, Journal of Sound and Vibration, 212(4), 703-719.

R. Kechroud, X. Antoine and A. Soulaimani (2000). Numerical accuracy of a Padetype non-reflecting boundary condition for the finite element solution of acoustic scattering problems at high-frequency, Int. J. Numer. Meth. Engng 2000; 00:16. http://www.acm.caltech.edu/antoine/articles/Pade-typeABC.pdf

S. M. Kirkup (1998-2007). ABEMFULL: Fortran codes for computing interior, exterior and modal acoustic problems in $2 D, 3 D$ and axisymmetric domains by the boundary element method http://www.boundary-element-method.com/acoustics

S. M. Kirkup (1999-2007). RIM3: Fortran code for computing the acoustic field exterior to a baffled panel by the Rayleigh Integral Method http://www.boundary-element-method.com/acoustics

S. M. Kirkup (2001-2007). BEMLAP: Fortran codes for computing interior, exterior and modal Laplace problems in 2D, 3D and axisymmetric domains by the boundary element method http://www.boundary-element-method.com/laplace 
S. M. Kirkup (2002-2007). BEMHELM: Fortran codes for computing interior, exterior and modal Helmholtz problems in 2D, 3D and axisymmetric domains by the boundary element method

http://www.boundary-element-method.com/helmholtz

S. M. Kirkup and A Thompson (2004). Simulation of the acoustic field produced by cavities using the Boundary Element Rayleigh Integral Method (BERIM) and its application to a horn loudspeaker, Presented at the International Congress on Sound and Vibration, St Petersburgh, 2004.

http://www.martin-audio.com/softtech/SIMULATION.pdf

S. M. Kirkup (2004-2007). BERIM3: Fortran code for computing the acoustic field exterior to a cavity by the Boundary Element- Rayleigh Integral Method (3D) http://www.boundary-element-method.com/acoustics

S. M. Kirkup (2007). DC capacitor simulation by the boundary element method, Academic Report AR-07-02, East Lancashire Academic Press, to appear in Communications in Numerical Methods in Engineering.

http://www.elihe.ac.uk/research

http://www.kirkup.info/papers

S. M. Kirkup and A Thompson (2007). Acoustic field of a horn loudspeaker simulation by the boundary element Rayleigh Integral method, Academic Report AR-07-03, East Lancashire Academic Press.

http://www.elihe.ac.uk/research

http://www.kirkup.info/papers

S. M. Kirkup (2007). BEMCD 200\%. ABEMFULL, BEMLAP and BEMHELM.

S. M. Kirkup. An Introduction to the Boundary Element Method: The BEMLAP Fortran Library, in preparation.

S. M. Kirkup. The Boundary Element Method in Acoustics second edition, in preparation. 
J. Kocbach, Pe. Lunde and M. Vestrheim (2000). Finite Element Modeling of Piezoceramic Disks including Radiation into a Fluid Medium. 23th Scandinavian Symposium on Physical Acoustics, Ustaoset. http://web.ift.uib.no/ jankoc/publ/ust2000/

J. Kobach (2000). Finite Element Modeling of Ultrasonic Piezoelectric Transducers Influence of geometry and material parameters on vibration, response functions and radiated field. University of Bergen, Department of Physics. http://web.ift.uib.no/ jankoc/thesis/diss.pdf

B-U Koo, B-C Lee and J-G Ih (1996). A non-singular Boundary Integral Equation for Acoustic Problems, Journal of Sound and Vibration, 192(1), 263-269. http://aclab.kaist.ac.kr/data/id11.pdf

I. Kosztin and K. Schulten (1997). Boundary Integral Method for Stationary States of Two-Dimensional Quantum Systems, International Journal of Modern Physics C, 8(2), 293-325. http://biophys.physics.missouri.edu/IK/PUBLICATIONS/Bound_Int_Meth_IJMPC/BEM.pdf

H. Lanzerath, H. Waller (1999). Computation of the time-history response of dynamic problems using the boundary element method and modal techniques, International Journal for Numerical Methods in Engineering, 45(7), 841 - 864.

A. Leblanc (2004) Thesis, Universit dArtois, Faculte des Sciences Appliquees de Bethune.

http://antlavie.free.fr/fichiers/recherche/these.pdf

Zhao Liu and Xiu Chen Xinzhao (2001). Combined boundary point analysis and experimental research of acoustic radiation problem caused by the vibrating machine, Chinese Journal of Mechanical Engineering, 37(11), 71-74. 
M.A.Paccini (2005) Developpment d'un modele elements finis 3D applique a la simulation d'operations chirurgicales des tissus mous, THESE pour obtenir le grade de Docteur de lEcole des Mines de Paris.

http://pastel.paristech.org/bib/archive/00001632/01/these_paccini.pdf

E. Perrey-Debain, J. Trevelyan, and P. Bettess (2003). Plane wave interpolation in direct collocation boundary element method for radiation and wave scattering: numerical aspects and applications, Journal of Sound and Vibration 261, 839858. http://www.dur.ac.uk/jon.trevelyan/JSV2003.pdf

E. Perrey-Debain, J. Trevelyan, and P. Bettess (2003). Use of wave boundary elements for acoustic computations, Journal of Computational Acoustics http://www.ma.man.ac.uk/ emmanuel/PUBLICATIONS/JCA2003.pdf

B. Pluymers, W. Desmet, D. Vandepitte and P Sas (2004) Application of an efficient wave-based prediction technique for the analysis of vibro-acoustic radiation problems, Journal of Computational and Applied Mathematics, 168(1-2), 353 - 364.

http://portal.acm.org/citation.cfm? $i d=1024610.1024645$

F. Polonio, T. Loyay, J.M. Parot and G. Gogu (2004). Acoustic radiation of an open structure: modelling and experiments. Acta Acustica united with Acustica, 90, 496511.

E. Premat Doctoral Thesis

http://csidoc.insa-lyon.fr/these/2000/premat/pagetitre.pdf

F. Schmidt (1998). An Alternative Derivation of the Exact DtN-Map on a Circle. Konrad-Zuse-Zentrum fur Informationstechnik Berlin http://citeseer.ist.psu.edu/440961.html

F. Schmidt (2001). Computation of Discrete Transparent Boundary Conditions for the 2D Helmholtz Equation, Optical and Quantum Electronics, 30(5-6).

http://citeseer.ist.psu.edu/564562.html 
J. Vanderkooy and D. J. Henwood (2006). Polar plots at low frequencies: The acoustic centre, Audio Engineering Society convention paper, 6784, 120th Convention, Paris, France.

A. VanderWyst, A. Christlieb, M. Sussman and I. D. Boyd. Simulation of Charge and Mass Distributions of Indium Droplets Created by Field Emission American Institute of Aeronautics and Astronautics http://hpcc.engin.umich.edu/CFD/research/NGPD/Publications/AIAA-06-3560.pdf

N. Vlahopoulos and S. T. Raveendra (1998). Formulation, implementation and validation of multiple connection and free edge constraints in an indirect Boundary Element Formulation. Journal of Sound and Vibration, 210(1), 137-152.

N. Vlahopoulos, Y. Kalish, S.T. Raveendra (1999). Development of an equivalent force method and an application in simulating the radiated noise from an operating diesel engine.Shock and Vibration 6(3), 113-123.

M. Willatzen, L.C. Lew Yan Voon (2003). Quantum Dot and Acoustic Enclosure Problems in Lens-Shaped Structures Springer Berlin / Heidelberg.

M. Willatzen, L. C. Lew Yan Voon (2004). Acoustic Cavity Modes in Lens-shaped structures, Journal of the Acoustical Society of America, 115(1) 84-90.

L. Wright, S.P. Robinson, V.F. Humphrey and G. Hayman (2005). The application of boundary element methods at NPL to near-field acoustic measurements on cylindrical surfaces. NPL Report DQL-AC 011.

http://publications.npl.co.uk/npl_web/pdf/dql_ac11.pdf

L Wrobel (2002). The Boundary Element Method - Applications in Thermo-Fluids Acoustics Vol. 1, John Wiley and Sons.

Shih-Jeh Wu, Ihyuan Kuo and K. Kirk Shung (2004). Boundary element simulation of backscattering properties for red blood with high frequency ultrasonic transducers, 
Ultrasonics, 43(3), 145-151.

Xiu Chen Xinzhao (2001). Acoustic radiation random vibration analysis of boundary points: The statistical boundary point analysis of acoustic radiation problem caused by the random vibrating body, Technical Acoustics 20(3), P.107-109.

S.I.Zaman (2000). A Comprehensive Review of Boundary Integral Formulations of Acoustic Scattering Problems, Department of Mathematics Statistics, College of Science, Sultan Qaboos University, P. O. Box 33, Al Khod 123, Muscat, Sultanate of Oman.

http://www.squ.edu.om/jstsqu/contents/papers/SpclRe2000.pdf/math-2.pdf

B. Zeitler (2005).A Comparative Study of Source Substitution Methods, Thesis. http://edocs.tu-berlin.de/diss/2005/zeitler_berndt.pdf

Reviews of the First Edition

Giora Rosenhouse, Journal of Computational Acoustics , 8(1), p257 (2000)

Applied Mechanics Reviews, Review 3R8, vol 52 no 3, B22-B23 (1999).

Mechanical Engineering, Software Exchange, May/Sep (1999).

Peter Juhl, International Journal of Acoustics and Vibration, 6(1), p47.

Marc Bonnet, Acta Acustica, 46, p392. 


\begin{tabular}{|l|l|}
\hline \hline URL & Brief details \\
\hline http://beteq.engineeringconferences.net & BETEQ conferences and proceedings \\
\hline www.boundary-element-method.com & BEM \\
\hline www.boundary-element-method.com/laplace & BEM for Laplace problems. \\
\hline www.boundary-element-method.com/helmholtz & BEM for Helmholtz problems \\
\hline www.boundary-element-method.com/acoustics & BEM for Acoustics problems \\
\hline www.boundary-element-method.com/other & Links to external BEM resources \\
\hline http://ejbe.libraries.rutgers.edu & Electronic Journal of Boundary Elements \\
\hline www.electromagnetics.info & electromagnetics \\
\hline www.finite-element-method.info & finite element method \\
\hline www.fortran.com & fortran resources \\
\hline http://gid.cimne.com & GID meshing software \\
\hline www.kirkup.info/papers & Stephen Kirkup's papers on-line \\
\hline www.mathworks.com & Mathworks : Matlab and Simulink \\
\hline www.nag.co.uk & NAg: Numerical Algorithms group \\
\hline www.nr.com & Numerical Recipes \\
\hline www.numerical-methods.com & numerical methods \\
\hline www.pafec.co.uk & FE for structural and BE for acoustics \\
\hline www.olemiss.edu/sciencenet/benet & boundary element resources network \\
\hline www.vibroacoustics.info & vibroacoustics \\
\hline
\end{tabular}

Table. Useful WWW resources on the BEM. 


\title{
Integral Equation Methods in Engineering
}

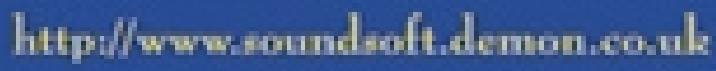

The bundary shment mathod (BEM) is a powerful iod in ompulationd

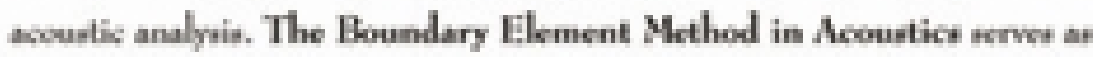

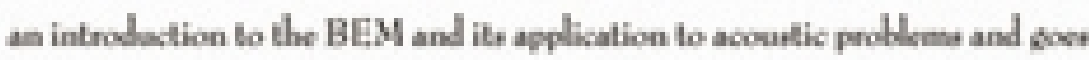

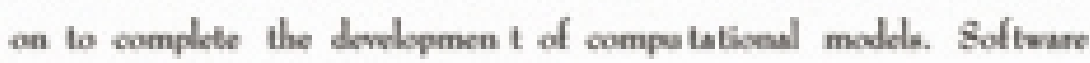
implomoning the mathale is mildth.

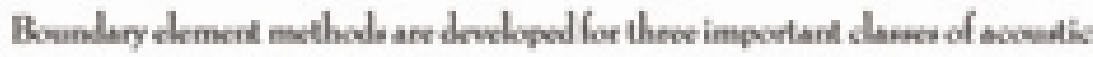

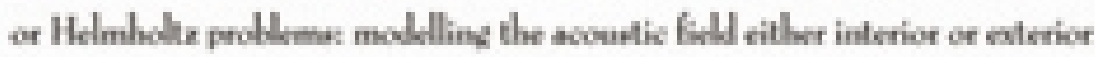

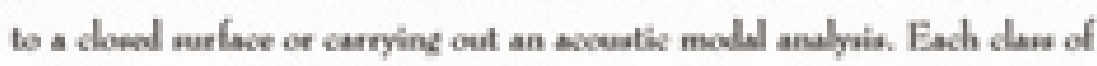

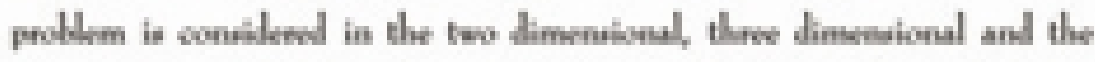

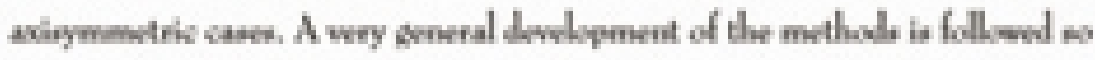

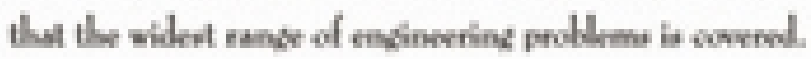

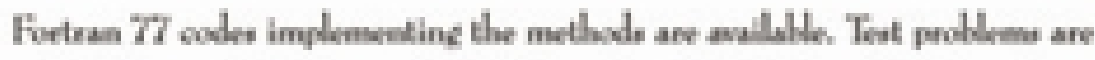

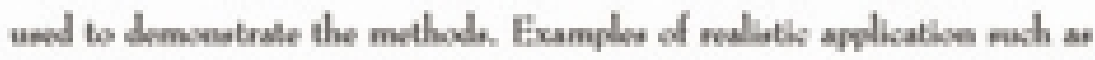

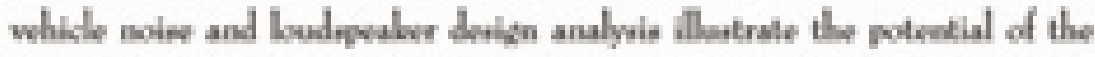

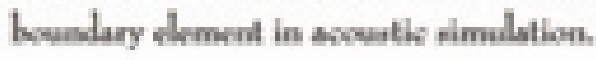

\section{Integrated Sound Software}

